\title{
Instanton Floer homology with Lagrangian boundary conditions
}

\author{
DIETMAR SALAMON \\ KATRIN WEHRHEIM
}

\begin{abstract}
In this paper we define instanton Floer homology groups for a pair consisting of a compact oriented 3-manifold with boundary and a Lagrangian submanifold of the moduli space of flat $S U(2)$-connections over the boundary. We carry out the construction for a general class of irreducible, monotone boundary conditions. The main examples of such Lagrangian submanifolds are induced from a disjoint union of handle bodies such that the union of the 3-manifold and the handle bodies is an integral homology 3-sphere. The motivation for introducing these invariants arises from our program for a proof of the Atiyah-Floer conjecture for Heegaard splittings. We expect that our Floer homology groups are isomorphic to the usual Floer homology groups of the closed 3-manifold in our main example and thus can be used as a starting point for an adiabatic limit argument.
\end{abstract}

57R58; 58J32

\section{Introduction}

In this paper we define instanton Floer homology groups for a pair consisting of a compact oriented 3-manifold with boundary and a Lagrangian submanifold of the moduli space of flat $\mathrm{SU}(2)$-connections over the boundary. We carry out the construction for a general class of irreducible, monotone boundary conditions. The main examples of such Lagrangian submanifolds are induced from a disjoint union of handle bodies such that the union of the 3-manifold and the handle bodies is an integral homology 3 -sphere. The motivation for introducing these invariants arises from our program for a proof of the Atiyah-Floer conjecture for Heegaard splittings [3; 28]. We expect that our Floer homology groups are isomorphic to the usual Floer homology groups (see Floer [14] and Donaldson [10]) of the closed 3-manifold in our main example and thus can be used as a starting point for an adiabatic limit argument as in Dostoglou and Salamon [12]. On the level of Euler characteristics, the Atiyah-Floer conjecture was proven by Taubes [30]. 
Floer homology groups for 3-manifolds with boundary were first constructed by Fukaya [16] with a different method. His setup uses nontrivial SO(3)-bundles and thus cannot immediately be used for the proof of the Atiyah-Floer conjecture where the bundles are necessarily trivial. Our approach is motivated by the construction of a Chern-Simons functional on 3-manifolds with boundary.

Let $Y$ be a compact oriented 3-manifold with boundary and denote

$$
\Sigma:=\partial Y, \quad \mathrm{G}:=\mathrm{SU}(2), \quad \mathfrak{g}:=\mathfrak{s u}(2), \quad\langle\xi, \eta\rangle:=-\operatorname{tr}(\xi \eta)
$$

for $\xi, \eta \in \mathfrak{g}$. While many of the results in this paper carry over to general compact Lie groups (and nontrivial bundles), our construction of Floer homology works in this form only for $\mathrm{G}=\mathrm{SU}(2)$ (where the bundles are necessarily trivial). The whole story also carries over to nontrivial $\mathrm{SO}(3)$-bundles, where the moduli spaces of flat connections are nonsingular and monotone, however, in this paper we restrict to the case $\mathrm{G}=\mathrm{SU}(2)$

The space $\mathcal{A}(\Sigma):=\Omega^{1}(\Sigma, \mathfrak{g})$ of connections on $\Sigma$ carries a natural symplectic form

$$
\omega(\alpha, \beta):=\int_{\Sigma}\langle\alpha \wedge \beta\rangle
$$

for $\alpha, \beta \in \mathrm{T}_{A} \mathcal{A}(\Sigma)=\Omega^{1}(\Sigma, \mathfrak{g})$, the action of the gauge group $\mathcal{G}(\Sigma):=\mathcal{C}^{\infty}(\Sigma, \mathrm{G})$ on $\mathcal{A}(\Sigma)$ is Hamiltonian, and the moment map is the curvature (see Atiyah and Bott [4]). The (singular) symplectic quotient is the moduli space

$$
M_{\Sigma}:=\mathcal{A}_{\text {flat }}(\Sigma) / \mathcal{G}(\Sigma)=\mathcal{A}(\Sigma) / / \mathcal{G}(\Sigma)
$$

of flat connections. We assume throughout that $\mathcal{L} \subset \mathcal{A}(\Sigma)$ is a gauge invariant, monotone, irreducible Lagrangian submanifold in the following sense.

(L1) $\mathcal{L}$ is a Fréchet submanifold of $\mathcal{A}(\Sigma)$, each tangent space $\mathrm{T}_{A} \mathcal{L}$ is a Lagrangian subspace of $\Omega^{1}(\Sigma, \mathfrak{g}), \mathcal{L} \subset \mathcal{A}_{\text {flat }}(\Sigma)$, and $\mathcal{L}$ is invariant under $\mathcal{G}(\Sigma)$.

(L2) The quotient of $\mathcal{L}$ by the based gauge group $\mathcal{G}_{z}(\Sigma)$ is compact, connected, simply connected, and $\pi_{2}\left(\mathcal{L} / \mathcal{G}_{z}(\Sigma)\right)=0$.

(L3) The zero connection is contained in $\mathcal{L}$ and is nondegenerate (as a critical point of the Chern-Simons functional). Moreover, every nontrivial flat connection $A \in \mathcal{A}(Y)$ with $\left.A\right|_{\Sigma} \in \mathcal{L}$ is irreducible.

A detailed explanation and a finite dimensional characterization of these conditions is given in Section 2. In particular, the assumptions imply that $\mathcal{L}$ descends to a (singular) Lagrangian submanifold $L:=\mathcal{L} / \mathcal{G}(\Sigma) \subset M_{\Sigma}$. If $H$ is a disjoint union of handlebodies with $\partial H=\bar{\Sigma}$ then the subset $\mathcal{L}_{H} \subset \mathcal{A}(\Sigma)$ of all flat connections on $\Sigma$ that extend to 
flat connections on $Y$ satisfies (L1) and (L2). It satisfies (L3) if and only if $Y \cup_{\Sigma} H$ is an integral homology 3 -sphere.

The space $\mathcal{A}(Y, \mathcal{L}):=\left\{A \in \mathcal{A}(Y)|A|_{\Sigma} \in \mathcal{L}\right\}$ of connections on $Y$ with boundary values in $\mathcal{L}$ carries a gauge invariant Chern-Simons functional

$$
\mathcal{C} \mathcal{S}_{\mathcal{L}}: \mathcal{A}(Y, \mathcal{L}) \rightarrow \mathbb{R} / 4 \pi^{2} \mathbb{Z},
$$

well defined up to an additive constant, whose differential is the usual Chern-Simons 1-form (see Section 2). The critical points are the flat connections in $\mathcal{A}(Y, \mathcal{L})$. If we fix a Riemannian metric $g$ on $Y$ then the gradient flow lines of the Chern-Simons functional with respect to the $L^{2}$ inner product are smooth maps $\mathbb{R} \rightarrow \mathcal{A}(Y): s \mapsto A(s)$ satisfying the differential equation

$$
\partial_{s} A+* F_{A}=0,\left.\quad A(s)\right|_{\Sigma} \in \mathcal{L} \quad \forall s \in \mathbb{R} .
$$

As in Floer's original work [14], the main idea is to use the solutions of (2) to construct a boundary operator on the chain complex generated by the gauge equivalence classes of the nontrivial flat connections in $\mathcal{A}_{\text {flat }}(Y, \mathcal{L})$. This defines the Floer homology groups $\operatorname{HF}(Y, \mathcal{L})$. To make this precise one needs perturbations that turn $\mathcal{C} \mathcal{S}_{\mathcal{L}}$ into a Morse function whose gradient flowlines satisfy Morse-Smale type transversality conditions.

We shall work with gauge invariant holonomy perturbations $h_{f}: \mathcal{A}(Y) \rightarrow \mathbb{R}$ as in Taubes [30], Floer [14] and Donaldson [10] (see Section 2 and Appendix D). The differential of $h_{f}$ has the form $\mathrm{d} h_{f}(A) \alpha=\int_{Y}\left\langle X_{f}(A) \wedge \alpha\right\rangle$ for a suitable map $X_{f}: \mathcal{A}(Y) \rightarrow \Omega^{2}(Y, \mathfrak{g})$. The space of gauge equivalence classes of critical points of the perturbed Chern-Simons functional $\mathcal{C S}_{\mathcal{L}}+h_{f}$ will be denoted by

$$
\mathcal{R}_{f}:=\left\{A \in \mathcal{A}(Y, \mathcal{L}) \mid F_{A}+X_{f}(A)=0\right\} / \mathcal{G}(Y)
$$

and the perturbed gradient flow lines are solutions of the boundary value problem

$$
\partial_{s} A+*\left(F_{A}+X_{f}(A)\right)=0,\left.\quad A(s)\right|_{\Sigma} \in \mathcal{L} \quad \forall s \in \mathbb{R} .
$$

The space of gauge equivalence classes of solutions of (3) that are asymptotic to $\left[A^{ \pm}\right] \in \mathcal{R}_{f}$ as $s$ tends to $\pm \infty$ will be denoted by $\mathcal{M}\left(A^{-}, A^{+} ; g, f\right)$. In the transverse case with irreducible limits $\left[A^{ \pm}\right] \neq 0$ this moduli space is a manifold whose local dimension near $[A] \in \mathcal{M}\left(A^{-}, A^{+} ; g, f\right)$ is given by the Fredholm index $\delta_{f}(A)$ of a suitable linearized operator. A crucial fact is the energy-index relation

$$
\delta_{f}(A)=\frac{2}{\pi^{2}} E_{f}(A)+\eta_{f}\left(A^{-}\right)-\eta_{f}\left(A^{+}\right)
$$

for the solutions of (3) with energy $E_{f}(A)=\int_{\mathbb{R}}\left\|\partial_{S} A\right\|_{L^{2}(Y)}^{2}$, and with a function $\eta_{f}: \mathcal{R}_{f} \rightarrow \mathbb{R}$. This is Floer's monotonicity formula; it follows from the fact that 
$\mathcal{L} / \mathcal{G}_{z}(\Sigma)$ is simply connected. The assumption on $\pi_{2}$ is only needed for the orientability of the moduli spaces.

Floer's original work corresponds to the case $\partial Y=\varnothing$. The object of the present paper is to show that all of Floer's ideas carry over to the case of nonempty boundary. The upshot is that, for a generic perturbation $h_{f}$, all critical points of $\mathcal{C} \mathcal{S}_{\mathcal{L}}+h_{f}$ are nondegenerate and so $\mathcal{R}_{f}$ is a finite set, and that, for every pair $\left[A^{ \pm}\right] \in \mathcal{R}_{f}$ the moduli space $\mathcal{M}^{1}\left(A^{-}, A^{+} ; g, f\right)$ of index 1 connecting trajectories consists of finitely many flow lines up to time shift. The monotonicity formula plays a central role in this finiteness theorem. As a result we obtain a Floer chain complex

$$
\mathrm{CF}_{*}(Y, \mathcal{L} ; f):=\bigoplus_{[A] \in \mathcal{R}_{f} \backslash[0]} \mathbb{Z}\langle A\rangle
$$

with boundary operator given by

$$
\partial\left\langle A^{-}\right\rangle:=\sum_{\left[A^{+}\right] \in \mathcal{R}_{f} \backslash[0]} \#\left(\mathcal{M}^{1}\left(A^{-}, A^{+} ; g, f\right) / \mathbb{R}\right)\left\langle A^{+}\right\rangle .
$$

Here the connecting trajectories are counted with appropriate signs determined by coherent orientations of the moduli spaces (Section 10). It then follows from gluing and compactness theorems (Sections 7 and 9) that $\partial^{2}=0$. The Floer homology groups are defined by

$$
\mathrm{HF}_{*}(Y, \mathcal{L} ; f, g):=\operatorname{ker} \partial / \operatorname{im} \partial .
$$

We shall prove that the Floer homology groups are independent of the choice of the metric $g$ and the perturbation $f$ used to define them (Section 11).

Remark 1.1 In the handle body case we expect the Floer homology groups $\operatorname{HF}\left(Y, \mathcal{L}_{H}\right)$ to be naturally isomorphic to the instanton Floer homology groups of the homology 3-sphere $Y \cup_{\Sigma} H$. The proof will be carried out elsewhere.

Remark 1.2 An interesting special case arises from a Heegaard splitting $M=$ $H_{0} \cup_{\Sigma} \bar{H}_{1}$ of a homology 3-sphere into two handle bodies $H_{i}$ with $\partial H_{i}=\Sigma$. We obtain the Floer homology groups $\mathrm{HF}_{*}\left([0,1] \times \Sigma, \mathcal{L}_{H_{0}} \times \mathcal{L}_{H_{1}}\right)$ from the following setup: The 3-manifold $Y:=[0,1] \times \Sigma$ has two boundary components $\partial Y=\bar{\Sigma} \sqcup \Sigma$, and attaching the disjoint union of the handle bodies $H:=H_{0} \sqcup \bar{H}_{1}$ yields the homology 3sphere $Y \cup_{\bar{\Sigma} \sqcup \Sigma} H \cong M$. The Lagrangian submanifold is $\mathcal{L}_{H_{0}} \times \mathcal{L}_{H_{1}} \cong \mathcal{L}_{H} \subset \mathcal{A}(\bar{\Sigma} \sqcup \Sigma)$. If this Floer homology is isomorphic to $\mathrm{HF}_{*}(M)$, as expected, then the proof of the Atiyah-Floer conjecture for $M$ reduces to an adiabatic limit argument as in [12] which identifies the symplectic Floer homology group of the pair of Lagrangian submanifolds $L_{H_{0}}, L_{H_{1}}$ of the singular symplectic manifold $M_{\Sigma}:=\mathcal{A}_{\text {flat }}(\Sigma) / \mathcal{G}(\Sigma)$ with the Floer 
homology groups $\operatorname{HF}\left([0,1] \times \Sigma, \mathcal{L}_{H_{0}} \times \mathcal{L}_{H_{1}}\right)$ defined in the present paper. Since $M_{\Sigma}$ is a singular space, this requires as a preliminary step the very definition of the symplectic Floer homology groups of $L_{H_{0}}$ and $L_{H_{1}}$ with $L_{H_{i}}:=\mathcal{L}_{H_{i}} / \mathcal{G}(\Sigma)$.

Remark 1.3 If $H_{0}, H_{1}, H_{2}$ are three handle bodies with boundary $\Sigma$ such that the manifold $M_{i j}:=H_{i} \cup_{\Sigma} \bar{H}_{j}$ is a homology 3-sphere for $i \neq j$, then there is a product morphism

$$
\mathrm{HF}_{*}\left(Y, \mathcal{L}_{H_{0}} \times \mathcal{L}_{H_{1}}\right) \times \mathrm{HF}_{*}\left(Y, \mathcal{L}_{H_{1}} \times \mathcal{L}_{H_{2}}\right) \rightarrow \mathrm{HF}_{*}\left(Y, \mathcal{L}_{H_{0}} \times \mathcal{L}_{H_{2}}\right),
$$

where $Y:=[0,1] \times \Sigma$. A key ingredient in the definition is the observation that (3) is the perturbed anti-self-duality equation for a connection on $\mathbb{R} \times Y$ in temporal gauge. Thus Equation (3) can be generalized to a 4-manifold $X$ with a boundary space-time splitting and tubular ends (Section 6). The definition of the product morphism will be based on the moduli space for the 4-manifold $X=\Delta \times \Sigma$, where $\Delta$ is a triangle (or rather a disc with three cylindrical ends attached). The details will be carried out elsewhere. We expect that our conjectural isomorphisms will intertwine the corresponding product structures on the symplectic and instanton Floer homologies.

The construction of the Floer homology groups in the present paper is based on the foundational analysis by Wehrheim $[33 ; 35 ; 36]$ and Mrowka and Wehrheim [23] for the solutions of the boundary value problem (2). In our exposition we follow the work of Floer [14] and Donaldson [10] and explain the details whenever new phenomena arise from our boundary value problem. Recall that the present Lagrangian boundary conditions are a mix of first order conditions (flatness of the restriction to $\partial Y$ ) and semi-global conditions (pertaining the holonomy on $\partial Y$ ), so they cannot be treated by standard nonlinear elliptic methods.

In Section 2 we recall the basic properties of the Chern-Simons functional on a $3-$ manifold with boundary and in Section 3 we discuss the Hessian and establish the basic properties of the linearized operator on $\mathbb{R} \times Y$. Section 4 examines the spectral flow and the determinant line bundle for operators over $S^{1} \times Y$. Section 5 establishes exponential decay on tubular ends. Section 6 sets up the Fredholm theory for general 4-manifolds with space-time splittings of the boundary and tubular ends. In the second half of the section we focus on the tube $\mathbb{R} \times Y$, examine the spectral flow, and prove monotonicity. Section 7 proves the compactness of the moduli spaces, based on [35; 36].

In Section 8 we establish transversality, using holonomy perturbations. The novel difficulty here is that we do not have a geometric description of the bubbling effect at the boundary. So, instead of a gluing theorem converse to bubbling, we use monotonicity and work inductively on the energy levels. The second difficulty is that we need to keep 
the support of the perturbations away from the boundary, since the techniques of [36] do not extend to the perturbed equation. As a result we cannot obtain an open and dense set of regular perturbations but - still sufficient - we find a regular perturbation up to index 7 near any given perturbation. In an appendix to this section we establish the relevant unique continuation results. In the process we reprove Taubes' unique continuation result [31] for anti-self-dual connections that vanish to infinite order at a point. This is needed to overcome difficulties arising from the nonlinear boundary conditions. After these preparations, the construction of the Floer homology follows the standard routine. For the gluing results in Section 9 we focus on the pregluing map and the Banach manifold setup for the inverse function theorem. In Section 10 we construct coherent orientations in the Lagrangian setting. The Floer homology groups are defined in Section 11.

There are several appendices where we review standard techniques and adapt them to our boundary value problems. Appendix A deals with the spectral flow for selfadjoint operator families with varying domains. Appendix B discusses the GelfandRobbin quotient, an abstract setting which relates self-adjoint operators with Lagrangian subspaces. These results are needed for the index calculations and orientations in Sections 4 and 6. Appendix $C$ reviews the Agmon-Nirenberg unique continuation technique used in Section 8. In Appendix D we discuss the basic analytic properties of the holonomy perturbations and prove a compactness result needed in Section 7. Appendix E deals with Lagrangian submanifolds in the space of connections. We construct an $L^{2}$-continuous trivialization of the tangent bundle $\mathrm{T} \mathcal{L}$, used in Sections 3 and 6, and a gauge invariant exponential map for $\mathcal{L}$, used in Section 9.

Notation We denote the spaces of smooth connections and gauge transformations on a manifold $Z$ by $\mathcal{A}(Z):=\Omega^{1}(Z, \mathfrak{g})$ and $\mathcal{G}(Z):=\mathcal{C}^{\infty}(Z, \mathrm{G})$. The gauge group $\mathcal{G}(Z)$ acts on $\mathcal{A}(Z)$ by $u^{*} A:=u^{-1} A u+u^{-1} \mathrm{~d} u$ and the gauge equivalence class of $A \in \mathcal{A}(Z)$ is denoted by $[A]$. A connection $A \in \mathcal{A}(Z)$ induces an exterior differential $\mathrm{d}_{A}: \Omega^{k}(Z, \mathfrak{g}) \rightarrow \Omega^{k+1}(Z, \mathfrak{g})$ via $\mathrm{d}_{A} \tau:=\mathrm{d} \tau+[A \wedge \tau]$. Here $[\cdot, \cdot]$ denotes the Lie bracket on $\mathfrak{g}$. The curvature of $A$ is the 2 -form $F_{A}:=\mathrm{d} A+A \wedge A$ and it satisfies $\mathrm{d}_{A} \mathrm{~d}_{A} \tau=\left[F_{A} \wedge \tau\right]$. The space of flat connections is denoted by $\mathcal{A}_{\text {flat }}(Z):=\{A \in$ $\left.\mathcal{A}(Z) \mid F_{A}=0\right\}$. Connections on $X=\mathbb{R} \times Y$ or other 4 -manifolds will be denoted by $\mathbb{A}$ or $\Xi$, whereas $A$ denotes a connection on a 3 -manifold $Y$ or a 2 -manifold $\Sigma$. We say that a connection $\mathbb{A}=A+\Phi \mathrm{d} s$ on $\mathbb{R} \times Y$ is in temporal gauge on $I \times Y$ if $\left.\Phi\right|_{I \times Y} \equiv 0$.

Acknowledgements The second author gratefully acknowledges support by the Swiss and US national science foundations and thanks IAS Princeton and FIM Zürich for their hospitality, during which most of this work was undertaken. 


\section{The Chern-Simons functional}

Let $Y$ be a compact oriented 3-manifold with boundary $\partial Y=\Sigma$ and $\mathrm{G}=\mathrm{SU}(2)$. The Chern-Simons 1 -form on $\mathcal{A}(Y)$ is defined by

$$
\alpha \mapsto \int_{Y}\left\langle F_{A} \wedge \alpha\right\rangle
$$

for $\alpha \in \mathrm{T}_{A} \mathcal{A}(Y)=\Omega^{1}(Y, \mathfrak{g})$. If $Y$ is closed, then (4) is the differential of the ChernSimons functional $\mathcal{C S}: \mathcal{A}(Y) \rightarrow \mathbb{R}$ given by

$$
\mathcal{C S}(A):=\frac{1}{2} \int_{Y}\left(\langle A \wedge \mathrm{d} A\rangle+\frac{1}{3}\langle A \wedge[A \wedge A]\rangle\right) .
$$

It changes by

$$
\mathcal{C S}(A)-\mathcal{C S}\left(u^{*} A\right)=4 \pi^{2} \operatorname{deg}(u)
$$

under a gauge transformation $u \in \mathcal{G}(Y)$; thus the Chern-Simons functional descends to a circle valued function $\mathcal{B}(Y):=\mathcal{A}(Y) / \mathcal{G}(Y) \rightarrow \mathbb{R} / 4 \pi^{2} \mathbb{Z}$ which will still be denoted by $\mathcal{C S}$. If $Y$ has nonempty boundary $\partial Y=\Sigma$, then the differential of (4) is the standard symplectic form $(1)$ on $\mathcal{A}(\Sigma)$. To obtain a closed 1-form we restrict the Chern-Simons 1 -form to a subspace of connections satisfying a Lagrangian boundary condition.

\section{Lagrangian submanifolds}

The relevant Lagrangian submanifolds of $\mathcal{A}(\Sigma)$ were studied in detail by Wehrheim [33, Section 4]. Following [33] we assume that $\mathcal{L} \subset \mathcal{A}(\Sigma)$ is a gauge invariant Lagrangian submanifold satisfying (L1). This condition can be rephrased as follows.

(L1) First, $\mathcal{L}$ is contained in $\mathcal{A}_{\text {flat }}(\Sigma)$ and is invariant under the action of $\mathcal{G}(\Sigma)$. Second, for some (and hence every) $p>2$ the $L^{p}$-closure of $\mathcal{L}$ is a Banach submanifold of the space of $L^{p}$-connections, $\mathcal{A}^{0, p}(\Sigma):=L^{p}\left(\Sigma, \mathrm{T}^{*} \Sigma \otimes \mathfrak{g}\right)$. Third, for every $A \in \mathcal{L}$ the tangent space $\mathrm{T}_{A} \mathcal{L} \subset \Omega^{1}(\Sigma, \mathfrak{g})$ is Lagrangian, ie

$$
\omega(\alpha, \beta)=0 \quad \forall \beta \in \mathrm{T}_{A} \mathcal{L} \quad \Longleftrightarrow \quad \alpha \in \mathrm{T}_{A} \mathcal{L}
$$

for every $\alpha \in \Omega^{1}(\Sigma, \mathfrak{g})$.

Let $\mathcal{L}^{0, p} \subset \mathcal{A}^{0, p}(\Sigma)$ denote the $L^{p}$-closure of $\mathcal{L}$. Then $\mathcal{L}=\mathcal{L}^{0, p} \cap \mathcal{A}(\Sigma)$ and the tangent space $\mathrm{T}_{A} \mathcal{L}$ of a smooth element $A \in \mathcal{L}$ - as in (L1) - is understood as the intersection of the Banach tangent space $\mathrm{T}_{A} \mathcal{L}^{0, p}$ with the space of smooth 1 -forms. This space is independent of $p>2$ and coincides with the space of derivatives of smooth 
paths in $\mathcal{L}$ passing through $A .{ }^{1}$ This follows from a finite dimensional characterization of the manifold property which we explain next.

A base point set is a finite set $z \subset \Sigma$ which intersects each component of $\Sigma$ in precisely one point. For every base point set $z$ the based gauge group $\mathcal{G}_{z}(\Sigma):=\{u \in$ $\mathcal{G}(\Sigma) \mid u(z) \equiv \mathbb{1}\}$ acts freely on $\mathcal{A}(\Sigma)$. Let $2 g:=\operatorname{dim} H_{1}(\Sigma)$ and pick $2 g$ loops in $\Sigma$ that generate $H_{1}(\Sigma)$ with base points chosen from $z$. The holonomy around these loops defines a map $\rho_{z}: \mathcal{A}_{\text {flat }} \rightarrow \mathrm{G}^{2 g}$ which is invariant under the action of the based gauge group $\mathcal{G}_{z}(\Sigma)$. If $\mathcal{L}$ is a gauge invariant subset of $\mathcal{A}_{\text {flat }}(\Sigma)$ then $\mathcal{L}^{0, p}$ is a Banach submanifold of $\mathcal{A}^{0, p}(\Sigma)$ if and only if the image $\rho_{z}(\mathcal{L}) \subset \mathrm{G}^{2 g}$ of the holonomy morphism is a smooth submanifold. There is however no well defined moment map for the action of $\mathcal{G}_{z}(\Sigma)$, so the symplectic structure does not descend to the quotient. On the other hand, the quotient $L:=\mathcal{L} / \mathcal{G}(\Sigma)$ has singularities in general, but it intersects the smooth part of the moduli space $M_{\Sigma}:=\mathcal{A}_{\text {flat }}(\Sigma) / \mathcal{G}(\Sigma)$ in a Lagrangian submanifold.

If $\mathcal{L}^{0, p} \subset \mathcal{A}^{0, p}(\Sigma)$ is a Lagrangian submanifold then $\mathcal{L}$ is gauge invariant if and only if $\mathcal{L} \subset \mathcal{A}_{\text {flat }}(\Sigma)$ [33, Section 4]. Condition (L1) implies that $\mathcal{L}$ is a totally real submanifold with respect to the Hodge $*$-operator for any metric on $\Sigma$, ie

$$
\Omega^{1}(\Sigma, \mathfrak{g})=\mathrm{T}_{A} \mathcal{L} \oplus * \mathrm{~T}_{A} \mathcal{L} \quad \forall A \in \mathcal{L} .
$$

The construction of Floer homology groups for the Chern-Simons 1-form will require the following additional assumptions on $\mathcal{L}$.

(L2) The quotient space $\mathcal{L} / \mathcal{G}_{z}(\Sigma)$ is compact, connected, simply connected, and $\pi_{2}\left(\mathcal{L} / \mathcal{G}_{z}(\Sigma)\right)=0$ for some (and hence every) base point set $z \subset \Sigma$.

(L3) The zero connection is contained in $\mathcal{L}$. It is nondegenerate in the sense that $\mathrm{d} \alpha=0 \Longleftrightarrow \alpha \in$ im d for every $\alpha \in \mathrm{T}_{0} \mathcal{A}(Y, \mathcal{L})$. Moreover, every flat connection in $\mathcal{A}(Y, \mathcal{L})$ that is not gauge equivalent to the zero connection is irreducible.

In (L2) the hypothesis that $\mathcal{L} / \mathcal{G}_{z}(\Sigma)$ is simply connected is needed to establish an energy-index relation for the Chern-Simons functional. The hypothesis $\pi_{2}\left(\mathcal{L} / \mathcal{G}_{z}(\Sigma)\right)=$ 0 is only used to orient the moduli spaces. It can be dropped if one wants to define Floer homology with $\mathbb{Z}_{2}$ coefficients. These two conditions imply that $\pi_{1}(\mathcal{L})$ is isomorphic to $\pi_{1}\left(\mathcal{G}_{z}(\Sigma)\right) \cong \pi_{1}(\mathcal{G}(\Sigma))$ and the map $\pi_{2}\left(\mathcal{G}_{z}(\Sigma)\right) \cong \pi_{2}(\mathcal{G}(\Sigma)) \rightarrow \pi_{2}(\mathcal{L})$ is surjective. To see this, note that $\mathcal{L}$ is a fiber bundle over the base $\mathcal{L} / \mathcal{G}_{z}(\Sigma)$ (see [33, Lemma 4.3]). In particular, (L2) implies that $\pi_{1}(\mathcal{L}) \cong \mathbb{Z}^{\pi_{0}(\Sigma)}$ since the fiber

\footnotetext{
${ }^{1}$ It is not clear whether one could also work with Hilbert submanifolds $\mathcal{L} \subset \mathcal{A}^{0,2}(\Sigma)$. This is connected to subtle questions concerning the gauge action at this Sobolev borderline; see Mrowka and Wehrheim [23].
}

Geometry $8 \mathcal{T}$ Topology, Volume 12 (2008) 
$\mathcal{G}_{z}(\Sigma)$ has fundamental group $\mathbb{Z}^{N}$ whenever $\Sigma$ has $N$ connected components. (For a connected component $\Sigma^{\prime}$ an isomorphism $\pi_{1}\left(\mathcal{G}_{z}\left(\Sigma^{\prime}\right)\right) \cong \mathbb{Z}$ is given by the degree of a $\operatorname{map} S^{1} \times \Sigma^{\prime} \rightarrow \mathrm{SU}(2) \cong S^{3}$.)

The main example of a Lagrangian submanifold of $\mathcal{A}(\Sigma)$ arises from the space of flat connections on a disjoint union $H$ of handle bodies ${ }^{2}$ with boundary $\partial H=\bar{\Sigma}$. Here $\bar{\Sigma}$ is the same manifold as $\Sigma$ but equipped with the opposite orientation. Given such a manifold $H$ define

$$
\mathcal{L}_{H}:=\left\{\left.\widetilde{A}\right|_{\Sigma} \mid \widetilde{A} \in \mathcal{A}_{\text {flat }}(H)\right\} .
$$

Lemma 2.1 Let $H$ be a disjoint union of handle bodies with $\partial Y=\bar{\Sigma}$. Then the following holds.

(i) $\mathcal{L}_{H}$ is a Lagrangian submanifold of $\mathcal{A}(\Sigma)$ that satisfies (L1) and (L2) and contains the zero connection.

(ii) The zero connection is nondegenerate if and only if $Y \cup H$ is a rational homology 3-sphere.

(iii) Every nontrivial flat connection in $\mathcal{A}_{\text {flat }}\left(Y, \mathcal{L}_{H}\right)$ is irreducible if and only if $Y \cup H$ is an integral homology 3 -sphere.

Proof That $\mathcal{L}_{H}$ satisfies (L1) was proved in [33, Lemma 4.6]. That $\mathcal{L}_{H}$ contains the zero connection is obvious. That it satisfies (L2) follows from the fact that the based holonomy map $\rho_{z}$ induces a homeomorphism from $\mathcal{L}_{H} / \mathcal{G}_{z}(\Sigma)$ to $\mathrm{G}^{g}$ with $\mathrm{G}=\mathrm{SU}(2)$ when $\Sigma$ is connected and has genus $g$, and that

$$
\mathcal{L}_{H_{1} \sqcup \ldots \sqcup H_{m}} / \mathcal{G}_{\left\{z_{1}, \ldots, z_{m}\right\}}\left(\Sigma_{1} \sqcup \ldots \sqcup \Sigma_{m}\right) \cong \mathcal{L}_{H_{1}} / \mathcal{G}_{z_{1}}\left(\Sigma_{1}\right) \times \ldots \times \mathcal{L}_{H_{m}} / \mathcal{G}_{z_{m}}\left(\Sigma_{m}\right)
$$

in the case of several connected components. This proves (i).

To prove (ii) we need to consider $\alpha \in \Omega^{1}(Y, \mathfrak{g})$ with $\mathrm{d} \alpha=0$. The linearized Lagrangian boundary condition on $\alpha$ is equivalent to the existence of an extension $\widetilde{\alpha} \in \Omega^{1}(Y \cup H, \mathfrak{g})$ with $\mathrm{d} \widetilde{\alpha}=0$. If $H^{1}(Y \cup H ; \mathbb{R})=0$ (or equivalently $H_{1}(Y \cup H ; \mathbb{Q})=0$ ), then any such 1 -form is exact on $Y \cup H$ and thus on $Y$. Conversely, if $\tilde{\alpha} \in$ ker d, then nondegeneracy implies $\left.\tilde{\alpha}\right|_{Y} \in$ imd and hence $\int_{\gamma} \tilde{\alpha}=0$ for every loop $\gamma \subset Y$. This implies that $\tilde{\alpha}$ is also exact on $Y \cup H$ since every loop in $Y \cup H$ is homotopic to a loop in $Y$. This proves (ii).

We prove (iii). Flat connections in $\mathcal{A}\left(Y, \mathcal{L}_{H}\right)$ can be identified with flat connections in $\mathcal{A}(Y \cup H)$. The gauge equivalence classes of irreducible but nontrivial connections

\footnotetext{
${ }^{2}$ A handle body is an oriented 3-manifold with boundary that is obtained from a 3-ball by attaching 1-handles. Equivalently, it admits a Morse function with exactly one minimum, no critical points of index 2 , and attaining its maximum on the boundary.
} 
are in one-to-one correspondence with nontrivial homomorphisms $\pi_{1}(Y \cup H) \rightarrow S^{1}$. These exist if and only if $H_{1}(Y \cup H ; \mathbb{Z}) \neq 0$.

\section{Lagrangian submanifolds and representations}

We characterize our Lagrangian submanifolds as subsets of the representation spaces for Riemann surfaces. For simplicity we assume first that $\Sigma$ is connected. Fix a base point $z \in \Sigma$ and choose based loops $\alpha_{1}, \ldots, \alpha_{g}, \beta_{1}, \ldots, \beta_{g}$ representing a standard set of generators ${ }^{3}$ of the fundamental group. The based holonomy around the loops $\alpha_{i}$ and $\beta_{i}$ gives rise to a map $\rho_{z}: \mathcal{A}(\Sigma) \rightarrow \mathrm{G}^{2 g}$. This map identifies the moduli space $M_{\Sigma}$ of flat connections with the quotient of $f^{-1}$ (11) by conjugation, where $f: \mathrm{G}^{2 g} \rightarrow \mathrm{G}$ is defined by

$$
f\left(x_{1}, \ldots, x_{g}, y_{1}, \ldots y_{g}\right):=\prod_{i=1}^{g} x_{i} y_{i} x_{i}^{-1} y_{i}^{-1} .
$$

The correspondence between flat connections and representations is reformulated in (a) and (b) below. Assertions (c) and (d) are the infinitesimal versions of these observations.

Remark 2.2 (a) Let $w=\left(x_{1}, \ldots, x_{g}, y_{1}, \ldots y_{g}\right) \in \mathrm{G}^{2 g}$. Then there exists a flat connection $A \in \mathcal{A}_{\text {flat }}(\Sigma)$ with $\rho_{z}(A)=w$ if and only if $f(w)=\mathbb{1}$.

(b) Let $A, A^{\prime} \in \mathcal{A}_{\text {flat }}(\Sigma)$. Then $A$ is gauge equivalent to $A^{\prime}$ if and only if $\rho_{z}(A)$ is conjugate to $\rho_{z}\left(A^{\prime}\right)$.

(c) Let $A \in \mathcal{A}_{\text {flat }}(\Sigma), w:=\rho_{z}(A)$, and $\widehat{w} \in \mathrm{T}_{w} \mathrm{G}^{2 g}$. Then $\mathrm{d} f(w) \hat{w}=0$ if and only if there exists an $\alpha \in \Omega^{1}(\Sigma, \mathfrak{g})$ such that $\mathrm{d}_{A} \alpha=0$ and $\mathrm{d} \rho_{z}(A) \alpha=\widehat{w}$.

(d) Let $A \in \mathcal{A}_{\text {flat }}(\Sigma)$ and $\alpha \in \Omega^{1}(\Sigma, \mathfrak{g})$. Denote $w:=\rho_{z}(A)$ and $\widehat{w}:=\mathrm{d} \rho_{z}(A) \alpha$. Then $\alpha \in \operatorname{imd}_{A}$ if and only if $\widehat{w}$ belongs to the image of the infinitesimal conjugate action $L_{w}: \mathfrak{g} \rightarrow \mathrm{T}_{w} \mathrm{G}^{2 g}$ given by $L_{w} \xi=\xi w-w \xi$.

While the identity element $\mathbb{1} \in \mathrm{G}$ is not a regular value of $f$, it follows from (c),(d) that the differential $\mathrm{d} \rho_{z}(A): \Omega^{1}(\Sigma, \mathfrak{g}) \rightarrow T_{w} \mathrm{G}^{2 g}$ at a flat connection $A \in \mathcal{A}_{\text {flat }}(\Sigma)$ identifies $H_{A}^{1}:=\operatorname{kerd}_{A} / \operatorname{imd}_{A}$ (the virtual tangent space of $M_{\Sigma}$ ) with the quotient kerd $f(w) / \operatorname{im} L_{w}$ at $w=\rho_{z}(A)$. The gauge invariant symplectic form (1) descends to $H_{A}^{1}$ and thus induces a symplectic form

$$
\begin{gathered}
\Omega_{w}: \operatorname{kerd} f(w) / \operatorname{im} L_{w} \times \operatorname{kerd} f(w) / \operatorname{im} L_{w} \rightarrow \mathbb{R} \\
\Omega_{w}\left(\widehat{w}, \hat{w}^{\prime}\right):=\int_{\Sigma}\left\langle\alpha \wedge \alpha^{\prime}\right\rangle,
\end{gathered}
$$

where the (infinitesimal) connections $A \in \mathcal{A}_{\text {flat }}(\Sigma)$ and $\alpha, \alpha^{\prime} \in \operatorname{kerd}_{A}$ are chosen such that $w=\rho_{z}(A), \widehat{w}=\mathrm{d} \rho_{z}(A) \alpha$, and $\widehat{w}^{\prime}=\mathrm{d} \rho_{z}(A) \alpha^{\prime}$. An explicit formula

\footnotetext{
${ }^{3}$ The standard generators of $\pi_{1}(\Sigma, z)$ satisfy the relation $\prod_{i=1}^{g} \alpha_{i} \beta_{i} \alpha_{i}^{-1} \beta_{i}^{-1}=\mathbb{1}$.
} 
for this symplectic form at the point $w=\left(x_{1}, \ldots, x_{g}, y_{1}, \ldots, y_{g}\right)$ on the vectors $\widehat{w}=\left(\xi_{1} x_{1}, \ldots, \xi_{g} x_{g}, \eta_{1} y_{1}, \ldots, \eta_{g} y_{g}\right), \widehat{w}^{\prime}=\left(\xi_{1}^{\prime} x_{1}, \ldots, \xi_{g}^{\prime} x_{g}, \eta_{1}^{\prime} y_{1}, \ldots, \eta_{g}^{\prime} y_{g}\right)$ is

$$
\begin{aligned}
\Omega_{w}\left(\widehat{w}, \widehat{w}^{\prime}\right)=\sum_{i=1}^{g}( & \left\langle\left(x_{i}^{-1} \xi_{i} x_{i}+x_{i}^{-1} \delta_{i} x_{i}-\delta_{i-1}\right), \eta_{i}^{\prime}\right\rangle \\
& \left.-\left\langle\left(y_{i}^{-1} \eta_{i} y_{i}+y_{i}^{-1} \delta_{i} y_{i}-\delta_{i-1}\right), \xi_{i}^{\prime}\right\rangle\right) .
\end{aligned}
$$

In this formula $\delta_{j}=-(\operatorname{dhol}(A) \alpha) \operatorname{hol}(A)^{-1}$ is the infinitesimal holonomy along the path $\prod_{i=1}^{j} \alpha_{i} \beta_{i} \alpha_{i}^{-1} \beta_{i}^{-1}$, ie

$$
\begin{gathered}
\delta_{j}=c_{j}^{-1} \delta_{c_{j}}+c_{j}^{-1} c_{j-1}^{-1} \delta_{c_{j-1}} c_{j}+\ldots+c_{j}^{-1} \ldots c_{1}^{-1} \delta_{c_{1}} c_{2} \ldots c_{j}, \\
c_{i}:=x_{i} y_{i} x_{i}^{-1} y_{i}^{-1}, \quad \delta_{c_{i}}:=x_{i} y_{i}\left(y_{i}^{-1} \xi_{i} y_{i}-\xi_{i}+\eta_{i}-x_{i}^{-1} \eta_{i} x_{i}\right) x_{i}^{-1} y_{i}^{-1} .
\end{gathered}
$$

One should compare this with the identities $f(w)=c_{1} \cdots c_{g}=\mathbb{1}$ and

$$
\mathrm{d} f(w)(\widehat{w})=c_{1} \ldots c_{g-1} \delta_{c_{g}}+c_{1} \ldots c_{g-2} \delta_{c_{g-1}} c_{g}+\ldots+\delta_{c_{1}} c_{2} \ldots c_{g}=0 .
$$

Combining these we see that $\delta_{c_{g}}=\mathbb{1 1}$. So on the torus $\Sigma=\mathbb{T}^{2}$ the formula simplifies to $\Omega_{w}\left(\widehat{w}, \widehat{w}^{\prime}\right)=\left\langle x^{-1} \xi x, \eta^{\prime}\right\rangle-\left\langle y^{-1} \eta y, \xi^{\prime}\right\rangle$. Moreover, if $T \subset \mathrm{G}$ is any circle and $w \in T^{2 g} \subset \mathrm{G}^{2 g}$ then the restriction of $\Omega_{w}$ to $\mathbb{R}^{2 g} \cong \mathrm{T}_{w} T^{2 g} \subset \operatorname{kerd} f(w)$ is the standard symplectic form on Euclidean space. By construction and assertions (ad) above, $\Omega$ descends to the symplectic form on the (singular) symplectic quotient $f^{-1}(11) / \mathrm{G} \cong M_{\Sigma}=\mathcal{A}(\Sigma) / / \mathcal{G}(\Sigma)$. In fact, one can verify directly that $\Omega$ is Ginvariant and that its kernel at each point is the tangent space to the $\mathrm{G}$-orbit. Thus, on the complement of the reducible set, $\Omega$ descends to a smooth symplectic structure on the $\mathrm{G}$-quotient.

In the case of the torus $\Sigma=\mathbb{T}^{2}$ all points of $f^{-1}(11)$ are reducible; in this case $M_{\mathbb{T}^{2}}$ can be identified with the quotient of the moduli space of flat $S^{1}$-connections by a residual $\mathbb{Z}_{2}$-action with four isolated fixed points $\{( \pm \mathbb{1 1}, \pm \mathbb{1 1})\}$ (corresponding to the same four points in $\mathrm{G}^{2}$ ). For a general surface $\Sigma$, the set of reducibles in $f^{-1}(\mathbb{1 1})$ is the union $\bigcup_{T \subset \mathrm{G}} T^{2 g}$ over all maximal tori $T \subset \mathrm{G}$. For $g>2$ this set has codimension $4 g-2>3 g$ in $\mathrm{G}^{2 g}$. So for a half dimensional submanifold $N \subset \mathrm{G}^{2 g}$ the set of irreducibles will always be dense in $N$. In the case of genus 2 the same is true if we require $\left.\Omega\right|_{\mathrm{T} N} \equiv 0$, since the codimension of the set of reducibles is $3 g$ but $N$ cannot intersect it in an open set since $\Omega$ is nondegenerate on each subtorus $T^{2 g}$ of the reducibles.

If $\Sigma$ has several connected components we fix a base point set $z \subset \Sigma$ and obtain the 2 -form $\Omega$ as sum of the 2 -forms of the connected components. We then have $M_{\Sigma} \cong f^{-1}(\mathbb{1}) / \mathrm{G}^{\pi_{0}(\Sigma)}$, where $\mathrm{G}^{\pi_{0}(\Sigma)}$ acts by conjugation with a fixed group element on each connected component and $f: \mathrm{G}^{2 g} \rightarrow \mathrm{G}^{\pi_{0}(\Sigma)}$ is the product of the relations (7) 
for each connected component. Now we can reformulate the assumptions (L1)-(L3) on the Lagrangian submanifolds $\mathcal{L} \subset \mathcal{A}(\Sigma)$ as follows: $\mathcal{L}=\rho_{z}^{-1}(N) \subset \mathcal{A}(\Sigma)$ is the preimage of a submanifold $N \subset \mathrm{G}^{2 g}$ satisfying the following conditions.

(L1) $N \subset f^{-1}(\mathbb{1 1}), N$ is invariant under $\mathrm{G}^{\pi_{0}(\Sigma)}, \operatorname{dim} N=3 g$, and $\left.\Omega\right|_{\mathrm{T} N} \equiv 0$.

(L2) $\quad N$ is compact, connected, simply connected, and $\pi_{2}(N)=0$.

(L3) $\quad N \cap \rho_{z}\left(\mathcal{A}_{\mathrm{flat}}(Y)\right)$ contains $(\mathbb{1 1}, \ldots, 11)$ as isolated point and does not contain any other reducible points (with respect to the conjugate action of $\mathrm{G}^{\pi_{0}}(\Sigma)$ ).

The above discussion of the reducible locus shows that, by condition (L1), the quotient $L:=N / G^{\pi_{0}(\Sigma)} \subset M_{\Sigma}$ is Lagrangian at a dense set of smooth points.

\section{The Chern-Simons functional}

Fix a compact, connected, oriented 3-manifold $Y$ with nonempty boundary $\partial Y=\Sigma$ and a gauge invariant, monotone, irreducible Lagrangian submanifold $\mathcal{L} \subset \mathcal{A}(\Sigma)$ satisfying (L1)-(L3). Then the restriction of the Chern-Simons 1-form (4) to the submanifold

$$
\mathcal{A}(Y, \mathcal{L}):=\left\{A \in \mathcal{A}(Y)|A|_{\Sigma} \in \mathcal{L}\right\}
$$

is closed. It is the differential of the circle valued Chern-Simons functional

$$
\mathcal{C} \mathcal{S}_{\mathcal{L}}: \mathcal{A}(Y, \mathcal{L}) \rightarrow \mathbb{R} / 4 \pi^{2} \mathbb{Z}
$$

given by $\mathcal{C S}_{\mathcal{L}}(A):=[\mathcal{C S}(A, B)]$, where

$$
\mathcal{C S}(A, B):=\frac{1}{2} \int_{Y}\left(\langle A \wedge \mathrm{d} A\rangle+\frac{1}{3}\langle A \wedge[A \wedge A]\rangle\right)-\frac{1}{2} \int_{0}^{1} \int_{\Sigma}\left\langle B(s) \wedge \partial_{s} B(s)\right\rangle \mathrm{d} s .
$$

Here $B:[0,1] \rightarrow \mathcal{L}$ is a smooth path satisfying $B(0)=\left.A\right|_{\Sigma}$ and $B(1)=0$.

Remark 2.3 Note that $\mathcal{C S}(A, B)$ is the value of the Chern-Simons functional on the connection $\tilde{A}$ on $\tilde{Y}:=Y \cup([0,1] \times \Sigma)$ given by $A$ on $Y$ and by $B$ on $[0,1] \times \Sigma$. Here we glue $\partial Y=\Sigma$ to $\{0\} \times \bar{\Sigma}$, and on the new boundary $\partial \tilde{Y}=\{1\} \times \Sigma$ we have $\tilde{A} \equiv 0$.

Lemma 2.4 (i) The Chern-Simons functional $\mathcal{C S}(A, B)$ is invariant under homotopies of $B$ with fixed endpoints.

(ii) If $u:[0,1] \rightarrow \mathcal{G}(\Sigma)$ satisfies $u(0)=u(1)=\mathbb{1}$ then

$$
\mathcal{C S}(A, B)-\mathcal{C S}\left(A, u^{*} B\right)=4 \pi^{2} \operatorname{deg} u .
$$


(iii) If $B_{0}, B_{1}:[0,1] \rightarrow \mathcal{L}$ are $t$ wo paths with $B_{0}(0)=B_{1}(0)$ and $B_{0}(1)=B_{1}(1)=0$ then there is a path $u:[0,1] \rightarrow \mathcal{G}(\Sigma)$ with $u(0)=u(1)=\mathbb{1}$ such that $B_{1}$ is homotopic to $u^{*} B_{0}$ (with fixed endpoints).

(iv) The circle valued function $\mathcal{C S}_{\mathcal{L}}: \mathcal{A}(Y, \mathcal{L}) \rightarrow \mathbb{R} / 4 \pi^{2} \mathbb{Z}$ descends to the quotient $\mathcal{B}(Y, \mathcal{L}):=\mathcal{A}(Y, \mathcal{L}) / \mathcal{G}(Y)$.

Proof The Chern-Simons functional is invariant under homotopies since

$$
\begin{aligned}
-\partial_{t} \mathcal{C S}\left(A, B_{t}\right) & =\frac{1}{2} \int_{0}^{1} \int_{\Sigma}\left(\left\langle\partial_{t} B_{t}(s) \wedge \partial_{s} B_{t}(s)\right\rangle+\left\langle B_{t}(s) \wedge \partial_{t} \partial_{s} B_{t}(s)\right\rangle\right) \mathrm{d} s \\
& =\int_{0}^{1} \int_{\Sigma}\left\langle\partial_{t} B_{t}(s) \wedge \partial_{s} B_{t}(s)\right\rangle \mathrm{d} s+\left[\int_{\Sigma}\left\langle B_{t}(s) \wedge \partial_{t} B_{t}(s)\right\rangle\right]_{0}^{1}
\end{aligned}
$$

for every smooth homotopy $B_{t}:[0,1] \rightarrow \mathcal{L}$ with fixed endpoints. The first term on the right is the symplectic form on $\partial_{t} B_{t}, \partial_{s} B_{t} \in \mathrm{T}_{B} \mathcal{L}$ and the second term vanishes since $\partial_{t} B_{t}(s)=0$ for $s=0,1$. Hence $\partial_{t} \mathcal{C S}\left(A, B_{t}\right)=0$. This proves (i).

To prove (ii), we abbreviate $S^{1}:=\mathbb{R} / \mathbb{Z}$, define $\tilde{u}: S^{1} \times \Sigma \rightarrow \mathrm{SU}(2)$ by $\tilde{u}(t, z):=$ $u(t)(z)$, and calculate

$$
\begin{aligned}
& 2\left(\mathcal{C S}(A, B)-\mathcal{C S}\left(A, u^{*} B\right)\right) \\
& =\int_{0}^{1} \int_{\Sigma}\left(\left\langle u^{*} B \wedge \partial_{S}\left(u^{*} B\right)\right\rangle-\left\langle B \wedge \partial_{S} B\right\rangle\right) \mathrm{d} s \\
& =\int_{0}^{1} \int_{\Sigma}\left(\left\langle B \wedge \mathrm{d}_{B}\left(\partial_{s} u \cdot u^{-1}\right)\right\rangle+\left\langle\mathrm{d} u \cdot u^{-1} \wedge\left(\partial_{s} B+\mathrm{d}_{B}\left(\partial_{s} u \cdot u^{-1}\right)\right)\right\rangle\right) \mathrm{d} s \\
& =\int_{0}^{1} \int_{\Sigma}\left(\left\langle B \wedge\left(2 \mathrm{~d}\left(\partial_{s} u \cdot u^{-1}\right)+\left[B, \partial_{s} u \cdot u^{-1}\right]\right)\right\rangle+\left\langle\mathrm{d} u \cdot u^{-1} \wedge \mathrm{d}\left(\partial_{s} u \cdot u^{-1}\right)\right\rangle\right) \mathrm{d} s \\
& =2 \int_{0}^{1} \int_{\Sigma}\left\langle F_{B} \wedge \partial_{S} u \cdot u^{-1}\right\rangle-\frac{1}{3} \int_{S^{1} \times \Sigma} \operatorname{tr}\left(\mathrm{d} \tilde{u} \cdot \tilde{u}^{-1} \wedge \mathrm{d} \tilde{u} \cdot \tilde{u}^{-1} \wedge \mathrm{d} \tilde{u} \cdot \tilde{u}^{-1}\right) \\
& =8 \pi^{2} \operatorname{deg} \tilde{u} .
\end{aligned}
$$

Here the first equation follows from the definitions, the second equation uses the formula $\partial_{s}\left(u^{*} B\right)=u^{-1}\left(\partial_{s} B+\mathrm{d}_{B}\left(\partial_{s} u \cdot u^{-1}\right)\right) u$, the third equation uses integration by parts in $s$ and the fact that $\mathrm{d} u(0)=\mathrm{d} u(1)=0$, the fourth equation uses the formula $d\left(\partial_{s} u \cdot u^{-1}\right)-\partial_{s}\left(d u \cdot u^{-1}\right)=\left[d u \cdot u^{-1}, \partial_{s} u \cdot u^{-1}\right]$ and integration by parts over $\Sigma$, and the last equation follows from the fact that $F_{B(s)}=0$ for every $s$ and that the standard volume form on $\mathrm{SU}(2)$ with integral 1 is $24 \pi^{2} \tilde{u}^{*} \operatorname{dvol}_{\mathrm{SU}(2)}=-\operatorname{tr}\left(\mathrm{d} \tilde{u} \cdot \tilde{u}^{-1} \wedge\right.$ $\left.\mathrm{d} \tilde{u} \cdot \tilde{u}^{-1} \wedge \mathrm{d} \tilde{u} \cdot \tilde{u}^{-1}\right)$. Thus we have proved (ii). 
To see (iii) note that the catenation of $-B_{0}$ and $B_{1}$ is a loop in $\mathcal{L}$ based at 0 . It is contractible in the base of the fibre bundle $\mathcal{G}_{z}(\Sigma) \hookrightarrow \mathcal{L} \rightarrow \mathcal{L} / \mathcal{G}_{z}(\Sigma)$ and hence it is homotopic to a loop $u:[0,1] \rightarrow \mathcal{G}_{z}(\Sigma)$ in the fibre based at $u(0)=u(1) \equiv \mathbb{1}$. Now the catenation of $B_{0},-B_{0}$, and $B_{1}$ is homotopic with fixed endpoints to $B_{1}$ on the one hand, and on the other hand to the catenation of $B_{0}$ with the loop $u^{*} 0$, which is also homotopic to $u^{*} B_{0}$.

It follows from (i-iii) that the map $(A, B) \rightarrow \mathcal{C S}(A, B)$ induces a circle valued function $\mathcal{C} \mathcal{S}_{\mathcal{L}}: \mathcal{A}(Y, \mathcal{L}) \rightarrow \mathbb{R} / 4 \pi^{2} \mathbb{Z}$. We prove that this function is invariant under gauge transformations. To see this we can use Remark 2.3 and extend any given $u \in \mathcal{G}(Y)$ to a gauge transformation $\tilde{u} \in \mathcal{G}(\tilde{Y})$ on $\tilde{Y}:=Y \cup([0,1] \times \Sigma)$ with $\left.\widetilde{u}\right|_{\tilde{Y}} \equiv \mathbb{1}$. Such an extension exists because $\mathcal{G}(\Sigma)$ is connected (which in turn follows from the fact that $\mathrm{G}=\mathrm{SU}(2)$ is connected, simply connected, and $\pi_{2}(\mathrm{G})=0$ ). Hence assertion (iv) follows from (5), which directly extends to gauge transformations that are trivial over the boundary. This proves the lemma.

Corollary 2.5 Let $B_{0} \in \mathcal{L}$ and $u:[0,1] \rightarrow \mathcal{G}(\Sigma)$ with $u(0)=u(1)=\mathbb{1 1}$. Then

$$
\int_{0}^{1} \int_{\Sigma}\left\langle u(s)^{*} B_{0} \wedge \partial_{s}\left(u(s)^{*} B_{0}\right)\right\rangle \mathrm{d} s=8 \pi^{2} \operatorname{deg}(u) .
$$

Proof The left hand side is twice the difference of the Chern-Simons functionals in Lemma 2.4 (ii).

\section{Perturbations}

We work with holonomy perturbations as in $[30 ; 14 ; 10]$. Let $\mathbb{D}:=\{z \in \mathbb{C}|| z \mid \leq 1\}$ be the closed unit disc and identify $S^{1}$ with $\mathbb{R} / \mathbb{Z}$, with the real coordinate denoted by $\theta$. Choose embeddings $\gamma_{i}: S^{1} \times \mathbb{D} \hookrightarrow \operatorname{int}(Y)$ for $i=1, \ldots, N$ such that the $\gamma_{i}$ coincide on a neighbourhood of $\{0\} \times \mathbb{D}$. We denote by $\rho_{i}: \mathbb{D} \times \mathcal{A}(Y) \rightarrow \mathrm{G}$ the map that assigns to a pair $(z, A)$ the holonomy of the connection $A$ around the loop $[0,1] \rightarrow Y: \theta \mapsto \gamma_{i}(\theta, z)$. Then the map $\rho=\left(\rho_{1}, \ldots, \rho_{N}\right): \mathbb{D} \times \mathcal{A}(Y) \rightarrow \mathrm{G}^{N}$ descends to a map between the quotient spaces $\mathbb{D} \times \mathcal{B}(Y) \rightarrow \mathrm{G}^{N} / \mathrm{G}$, where the action of $\mathrm{G}$ on $\mathrm{G}^{N}$ is by simultaneous conjugation and $\mathcal{B}(Y):=\mathcal{A}(Y) / \mathcal{G}(Y)$.

Now every smooth function $f: \mathbb{D} \times \mathrm{G}^{N} \rightarrow \mathbb{R}$ that is invariant under conjugation and vanishes near the boundary induces a gauge invariant perturbation $h_{f}: \mathcal{A}(Y) \rightarrow \mathbb{R}$ given by

$$
h_{f}(A):=\int_{\mathbb{D}} f(z, \rho(z, A)) \mathrm{d}^{2} z
$$


The differential $\mathrm{d} h_{f}(A): \mathrm{T}_{A} \mathcal{A}(Y) \rightarrow \mathbb{R}$ has the form

$$
\mathrm{d} h_{f}(A) \alpha=\int_{Y}\left\langle X_{f}(A) \wedge \alpha\right\rangle,
$$

where $X_{f}: \mathcal{A}(Y) \rightarrow \Omega^{2}(Y, \mathfrak{g})$ is a smooth map satisfying

$$
\mathrm{d}_{A} X_{f}(A)=0, \quad X_{f}\left(u^{*} A\right)=u^{-1} X_{f}(A) u, \quad \mathrm{~d} X_{f}(A) \mathrm{d}_{A} \xi=\left[X_{f}(A), \xi\right]
$$

for $A \in \mathcal{A}(Y), u \in \mathcal{G}(Y), \xi \in \Omega^{0}(Y, \mathfrak{g})$. This follows from the gauge invariance of $h_{f}$ (see Appendix D). Since $\mathrm{d} X_{f}(A)$ is the Hessian of $h_{f}$ we have

$$
\int_{Y}\left\langle\mathrm{~d} X_{f}(A) \alpha \wedge \beta\right\rangle=\int_{Y}\left\langle\mathrm{~d} X_{f}(A) \beta \wedge \alpha\right\rangle .
$$

Moreover, $X_{f}(A)$ is supported in the union of the thickened loops $\gamma_{i}\left(S^{1} \times \mathbb{D}\right)$ and hence in the interior of $Y$.

\section{Critical points}

The critical points of the perturbed Chern-Simons functional $\mathcal{C} \mathcal{S}_{\mathcal{L}}+h_{f}$ are the solutions $A \in \mathcal{A}(Y)$ of the equation

$$
F_{A}+X_{f}(A)=0,\left.\quad A\right|_{\Sigma} \in \mathcal{L} .
$$

Let $\operatorname{Crit}\left(\mathcal{C S}_{\mathcal{L}}+h_{f}\right)$ denote the set of critical points and abbreviate

$$
\mathcal{R}_{f}:=\operatorname{Crit}\left(\mathcal{C S}_{\mathcal{L}}+h_{f}\right) / \mathcal{G}(Y) .
$$

Associated to every critical point $A \in \mathcal{A}(Y, \mathcal{L})$ of $\mathcal{C S}_{\mathcal{L}}+h_{f}$ is a twisted deRham complex

where

$$
\begin{aligned}
\Omega^{0}(Y, \mathfrak{g}) \stackrel{\mathrm{d}_{A}}{\longrightarrow} \Omega_{\mathrm{T}_{A} \mathcal{L}}^{1}(Y, \mathfrak{g}) \stackrel{\mathrm{d}_{A}+\mathrm{d} X_{f}(A)}{\longrightarrow} \Omega_{0}^{2}(Y, \mathfrak{g}) \stackrel{\mathrm{d}_{A}}{\longrightarrow} \Omega^{3}(Y, \mathfrak{g}), \\
\Omega_{\mathrm{T}_{A} \mathcal{L}}^{1}(Y, \mathfrak{g}):=\left\{\alpha \in \Omega^{1}(Y, \mathfrak{g})|\alpha|_{\Sigma} \in T_{\left.A\right|_{\Sigma}} \mathcal{L}\right\}, \\
\Omega_{0}^{2}(Y, \mathfrak{g}):=\left\{\tau \in \Omega^{2}(Y, \mathfrak{g})|\tau|_{\Sigma}=0\right\} .
\end{aligned}
$$

The first operator in this complex is the infinitesimal action of the gauge group, the second corresponds to the Hessian of the Chern-Simons functional, and the third to the Bianchi identity. A critical point $A$ is called irreducible if the cohomology group $H_{A}^{0}$ of (12) vanishes, ie the operator $\mathrm{d}_{A}: \Omega^{0}(Y, \mathfrak{g}) \rightarrow \Omega^{1}(Y, \mathfrak{g})$ is injective. It is called nondegenerate if the cohomology group $H_{A, f}^{1}$ vanishes, ie for every $\alpha \in \mathrm{T}_{A} \mathcal{A}(Y, \mathcal{L})$ we have

$$
\mathrm{d}_{A} \alpha+\mathrm{d} X_{f}(A) \alpha=0 \quad \Longleftrightarrow \quad \alpha \in \operatorname{imd}_{A} .
$$


This nondegeneracy means that the Hessian of the Chern-Simons functional is nondegenerate on a local slice of the gauge action. In Section 8 we will prove that for a generic perturbation every critical point is nondegenerate, ie $\mathcal{C} \mathcal{S}_{\mathcal{L}}+h_{f}$ induces a Morse function on the quotient $\mathcal{B}(Y, \mathcal{L})$.

\section{Gradient flow lines}

Fix a metric $g$ on $Y$. Then a negative gradient flow line of the perturbed functional $\mathcal{C S}_{\mathcal{L}}+h_{f}$ is a connection $\mathbb{A} \in \mathcal{A}(\mathbb{R} \times Y)$ in temporal gauge, represented by a smooth path $\mathbb{R} \rightarrow \mathcal{A}(Y): s \mapsto A(s)$ that satisfies the boundary value problem

$$
\partial_{s} A+*\left(F_{A}+X_{f}(A)\right)=0,\left.\quad A(s)\right|_{\Sigma} \in \mathcal{L} \quad \forall s \in \mathbb{R} .
$$

The energy of a solution is

$$
E_{f}(\mathbb{A})=\frac{1}{2} \int_{\mathbb{R} \times Y}\left(\left|\partial_{s} A\right|^{2}+\left|F_{A}+X_{f}(A)\right|^{2}\right) .
$$

In Section 5 we prove that (in the nondegenerate case) a solution $\mathbb{A}$ of (14) has finite energy if and only if there exist critical points $A^{+}, A^{-} \in \operatorname{Crit}\left(\mathcal{C S}_{\mathcal{L}}+h_{f}\right)$ such that $A(s)$ converges exponentially to $A^{ \pm}$as $s$ tends to $\pm \infty$. Denote the moduli space of connecting trajectories from $\left[A^{-}\right]$to $\left[A^{+}\right]$by

$$
\mathcal{M}\left(A^{-}, A^{+}\right):=\left\{\begin{array}{l|l}
\mathbb{A} \in \mathcal{A}^{\mathrm{tmp}}(\mathbb{R} \times Y) & \begin{array}{c}
(14), E_{f}(\mathbb{A})<\infty, \\
\lim _{s \rightarrow \pm \infty} A(s) \in\left[A^{ \pm}\right]
\end{array}
\end{array}\right\} / \mathcal{G}(Y),
$$

where $\mathcal{A}^{\mathrm{tmp}}(\mathbb{R} \times Y)$ denotes the space of connections on $\mathbb{R} \times Y$ in temporal gauge. The analogue of Equation (14) for connections $\mathbb{A}=\Phi \mathrm{d} s+A$ that are not in temporal gauge is

$$
\partial_{s} A-\mathrm{d}_{A} \Phi+*\left(F_{A}+X_{f}(A)\right)=0,\left.\quad A(s)\right|_{\Sigma} \in \mathcal{L} \quad \forall s \in \mathbb{R} .
$$

This equation can be written in the form

$$
F_{\mathbb{A}}+X_{f}(\mathbb{A})+*\left(F_{\mathbb{A}}+X_{f}(\mathbb{A})\right)=0,\left.\quad \mathbb{A}\right|_{\{s\} \times \partial Y} \in \mathcal{L} \quad \forall s \in \mathbb{R},
$$

where $X_{f}(\mathbb{A})(s, y)=X_{f}(A(s))(y)$. In this form it generalizes to 4-manifolds with a space time of the boundary and tubular ends.

The moduli space $\mathcal{M}\left(A^{-}, A^{+}\right)$can also be described as the quotient of the space of all finite energy solutions of (15) in temporal gauge outside of a compact set that converge to $A^{ \pm}$as $s \rightarrow \pm \infty$. In this case the gauge group consists of gauge transformations that are independent of $s$ outside of a compact set and preserve $A^{ \pm}$at the ends. The study of the moduli space is based on the analysis of the linearized operator for Equation (15). As a first step we examine the Hessian of the Chern-Simons functional. 


\section{The Hessian}

In this section we establish the basic analytic properties of the Hessian of the ChernSimons functional and draw some conclusions on the structure of the set of critical points and the linearized operator of the gradient flow lines.

We continue the notation of Section 2. The augmented Hessian of the perturbed Chern-Simons functional at a connection $A \in \mathcal{A}(Y, \mathcal{L})$ is the operator

$$
\mathcal{H}_{A}:=\left(\begin{array}{cc}
* \mathrm{~d}_{A}+* \mathrm{~d} X_{f}(A) & -\mathrm{d}_{A} \\
-\mathrm{d}_{A}^{*} & 0
\end{array}\right) .
$$

The additional terms $-\mathrm{d}_{A}$ and $-\mathrm{d}_{A}^{*}$ arise from a local slice condition. Think of $\mathcal{H}_{A}$ as an unbounded operator on the Hilbert space $L^{2}\left(Y, \mathrm{~T}^{*} Y \otimes \mathfrak{g}\right) \times L^{2}(Y \otimes \mathfrak{g})$ with dense domain

$$
\operatorname{dom} \mathcal{H}_{A}:=\left\{(\alpha, \varphi) \in W^{1,2}\left(Y, \mathrm{~T}^{*} Y \otimes \mathfrak{g}\right) \times W^{1,2}(Y, \mathfrak{g})|* \alpha|_{\partial Y}=0,\left.\alpha\right|_{\partial Y} \in \mathrm{T}_{A} \mathcal{L}\right\} .
$$

Here we abbreviate $\mathrm{T}_{A} \mathcal{L}:=\mathrm{T}_{\left.A\right|_{\Sigma}} \mathcal{L}$ for $A \in \mathcal{A}(Y, \mathcal{L})$.

The operator $\mathcal{H}_{A}$ is symmetric: for $\alpha, \beta \in \Omega^{1}(Y, \mathfrak{g})$ and $\varphi, \psi \in \Omega^{0}(Y, \mathfrak{g})$

$$
\begin{aligned}
&\left\langle\mathcal{H}_{A}(\alpha, \varphi),(\beta, \psi)\right\rangle_{L^{2}}-\left\langle(\alpha, \varphi), \mathcal{H}_{A}(\beta, \psi)\right\rangle_{L^{2}} \\
&= \int_{Y}\left\langle\left(\mathrm{~d}_{A} \alpha+\mathrm{d} X_{f}(A) \alpha-* \mathrm{~d}_{A} \varphi\right) \wedge \beta\right\rangle+\int_{Y}\left\langle\left(\mathrm{~d}_{A} * \alpha\right) \wedge \psi\right\rangle \\
&-\int_{Y}\left\langle\alpha \wedge\left(\mathrm{d}_{A} \beta+\mathrm{d} X_{f}(A) \beta-* \mathrm{~d}_{A} \psi\right)\right\rangle-\int_{Y}\left\langle\varphi \wedge\left(\mathrm{d}_{A} * \beta\right)\right\rangle \\
&= \int_{\partial Y}\langle\alpha \wedge \beta\rangle-\int_{\partial Y}\langle\varphi, * \beta\rangle+\int_{\partial Y}\langle * \alpha, \psi\rangle .
\end{aligned}
$$

If both $(\alpha, \varphi)$ and $(\beta, \psi)$ belong to the domain of $\mathcal{H}_{A}$, then the boundary conditions guarantee that the last three integrals vanish. In particular, $\int_{\partial Y}\langle\alpha \wedge \beta\rangle$ is the symplectic form on $\left.\alpha\right|_{\partial Y},\left.\beta\right|_{\partial Y} \in \mathrm{T}_{A} \mathcal{L}$. An $L^{2}$-estimate for the Hessian is obtained from the following elementary calculation: If $(\alpha, \varphi) \in \operatorname{dom} \mathcal{H}_{A}$ then

$$
\begin{aligned}
\left\|\mathcal{H}_{A}(\alpha, \varphi)\right\|_{L^{2}}^{2} & =\left\|* \mathrm{~d}_{A} \alpha-\mathrm{d}_{A} \varphi\right\|_{L^{2}}^{2}+\left\|\mathrm{d}_{A}^{*} \alpha\right\|_{L^{2}}^{2} \\
& =\left\|\mathrm{d}_{A} \alpha\right\|_{L^{2}}^{2}+\left\|\mathrm{d}_{A} \varphi\right\|_{L^{2}}^{2}+\left\|\mathrm{d}_{A}^{*} \alpha\right\|_{L^{2}}^{2}-2 \int_{Y}\left\langle\alpha \wedge\left[F_{A}, \varphi\right]\right\rangle \\
& \geq \delta\|(\alpha, \varphi)\|_{W^{1,2}}^{2}-C\|(\alpha, \varphi)\|_{L^{2}}^{2}
\end{aligned}
$$

Here the second equation follows from integration by parts. The inequality, with suitable constants $\delta>0$ and $C$, follows from the Cauchy-Schwarz inequality and [34, Theorem 5.1] with $p=2$. The resulting estimate $\|(\alpha, \varphi)\|_{W^{1,2}} \leq \delta^{-1 / 2}\left\|\mathcal{H}_{A}(\alpha, \varphi)\right\|_{L^{2}}+$ 
$(C / \delta)^{1 / 2}\|(\alpha, \varphi)\|_{L^{2}}$ implies that $\mathcal{H}_{A}$ has a finite dimensional kernel and a closed image. In Proposition 3.1 below (which is the main result of this section) we will identify the cokernel $\left(\operatorname{im} \mathcal{H}_{A}\right)^{\perp}$ ) with the kernel and thus prove that the Hessian is a Fredholm operator and self-adjoint. We moreover establish the estimate for the Hessian in general $W^{k, p}$-Sobolev spaces. This will be used in the analysis of the linearized operator on $\mathbb{R} \times Y$ and for the exponential decay analysis.

Proposition 3.1 (i) $\mathcal{H}_{A}$ is a self-adjoint Fredholm operator.

(ii) For every $A \in \mathcal{A}(Y, \mathcal{L})$ and every integer $k \geq 0$ and every $p>1$ there exists a constant $C$ such that the following holds. If $(\alpha, \varphi) \in \operatorname{dom} \mathcal{H}_{A}$ and $\mathcal{H}_{A}(\alpha, \varphi)$ is of class $W^{k, p}$, then $(\alpha, \varphi)$ is of class $W^{k+1, p}$ and

$$
\|(\alpha, \varphi)\|_{W^{k+1, p}(Y)} \leq C\left(\left\|\mathcal{H}_{A}(\alpha, \varphi)\right\|_{W^{k, p}(Y)}+\|(\alpha, \varphi)\|_{L^{p}(Y)}\right) .
$$

(iii) If $F_{A}+X_{f}(A)=0$ then ker $\mathcal{H}_{A}=H_{A, f}^{1} \times H_{A}^{0}$, where

$$
\begin{aligned}
H_{A}^{0} & :=\operatorname{ker} \mathrm{d}_{A} \subset \Omega^{0}(Y, \mathfrak{g}), \\
H_{A, f}^{1} & :=\operatorname{ker}\left(\mathrm{d}_{A}+\mathrm{d} X_{f}(A)\right) \cap \operatorname{kerd}_{A}^{*} \subset \Omega_{A}^{1}(Y, \mathfrak{g}), \\
\Omega_{A}^{1}(Y, \mathfrak{g}) & :=\left\{\alpha \in \Omega^{1}(Y, \mathfrak{g})|* \alpha|_{\partial Y}=0,\left.\alpha\right|_{\partial Y} \in \mathrm{T}_{A} \mathcal{L}\right\} .
\end{aligned}
$$

Definition 3.2 Let $A \in \mathcal{A}(Y, \mathcal{L})$ be a critical point of the perturbed Chern-Simons functional, ie $F_{A}+X_{f}(A)=0$. The connection $A$ is called nondegenerate if $H_{A, f}^{1}=0$; it is called irreducible if $H_{A}^{0}=0$.

Remark 3.3 (i) The vector spaces $H_{A}^{0}$ and $H_{A, f}^{1}$ in Proposition 3.1 are isomorphic to the first two cohomology groups in the complex (12); they are the spaces of harmonic representatives. Hence a critical point $A \in \mathcal{A}(Y, \mathcal{L})$ is nondegenerate in the sense of Definition 3.2 if and only if it satisfies (13).

(ii) Hypothesis (L3) says that $A=0$ is nondegenerate for the zero perturbation $f=0$. Since the differential $\mathrm{d} X_{f}(A)$ vanishes at $A=0$ for every $f$ (see Appendix D) it follows that $A=0$ is nondegenerate for any perturbation.

The proof of Proposition 3.1 requires some preparation. First, we need to introduce norms for the boundary terms in the upcoming estimates. Let $p^{*}$ denote the dual exponent of $p$ given by $1 / p+1 / p^{*}=1$. We define the following norms (which strictly speaking depend on $Y$ ) for a smooth function $\varphi: \Sigma=\partial Y \rightarrow \mathfrak{g}$

$$
\begin{aligned}
& \|\varphi\|_{b W^{1-1 / p, p(\Sigma)}}:=\inf \left\{\|\tilde{\varphi}\|_{W^{1, p}(Y)}|\tilde{\varphi}|_{\Sigma}=\varphi\right\},
\end{aligned}
$$

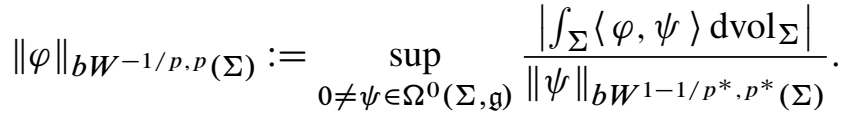


For a 2 -form $\tau \in \Omega^{2}(\Sigma, \mathfrak{g})$ the corresponding norms are understood as the norms of the function $* \tau \in \Omega^{0}(\Sigma, \mathfrak{g})$. The following estimates for these boundary Sobolev norms will be useful.

Lemma 3.4 For $A \in \mathcal{A}(Y)$ and $\alpha \in \Omega^{1}(Y, \mathfrak{g})$ we have

$$
\left\|\mathrm{d}_{\left.A\right|_{\Sigma}}\left(\left.\alpha\right|_{\Sigma}\right)\right\|_{b W^{-1 / p, p}(\Sigma)}=\sup _{0 \neq \psi \in \Omega^{0}(Y, \mathfrak{g})} \frac{\left|\int_{Y}\left(\left\langle\mathrm{~d}_{A} \alpha \wedge \mathrm{d}_{A} \psi\right\rangle-\left\langle\alpha \wedge\left[F_{A}, \psi\right]\right\rangle\right)\right|}{\|\psi\|_{W^{1, p^{*}}(Y)}} .
$$

Moreover, if $A \in \mathcal{A}(Y, \mathcal{L})$ is a critical point of $\mathcal{C S}_{\mathcal{L}}+h_{f}$ then

$$
\left\|\mathrm{d}_{\left.A\right|_{\Sigma}}\left(\left.\alpha\right|_{\Sigma}\right)\right\|_{b W^{-1 / p, p}(\Sigma)} \leq\left(1+\|A\|_{L^{\infty}(Y)}\right)\left\|\mathrm{d}_{A} \alpha+\mathrm{d} X_{f}(A) \alpha\right\|_{L^{p}(Y)} .
$$

Proof By definition we have

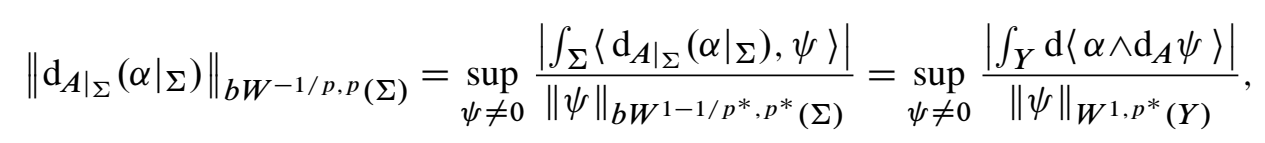

where the supremum runs over all nonzero functions $\psi \in \Omega^{0}(Y, \mathfrak{g})$. Now the first identity follows from $\mathrm{d}\left\langle\alpha \wedge \mathrm{d}_{A} \psi\right\rangle=\left\langle\mathrm{d}_{A} \alpha \wedge \mathrm{d}_{A} \psi\right\rangle-\left\langle\alpha \wedge\left[F_{A}, \psi\right]\right\rangle$. If $A \in \mathcal{A}(Y, \mathcal{L})$ is a critical point of $\mathcal{C} \mathcal{S}_{\mathcal{L}}+h_{f}$ then $F_{A}+X_{f}(A)=0$ and hence

$$
\begin{aligned}
\left\|\mathrm{d}_{\left.A\right|_{\Sigma}}\left(\left.\alpha\right|_{\Sigma}\right)\right\|_{b W^{-1 / p, p}(\Sigma)} & =\sup _{\psi \neq 0} \frac{\left|\int_{Y}\left\langle\left(\mathrm{~d}_{A} \alpha+\mathrm{d} X_{f}(A) \alpha\right) \wedge \mathrm{d}_{A} \psi\right\rangle\right|}{\|\psi\|_{W^{1, p^{*}}(Y)}} \\
& \leq\left(1+\|A\|_{L^{\infty}(Y)}\right)\left\|\mathrm{d}_{A} \alpha+\mathrm{d} X_{f}(A) \alpha\right\|_{L^{p}(Y)},
\end{aligned}
$$

where we have used (10) and (11). This proves the lemma.

The following lemma provides the basic estimates for Proposition 3.1. The first part is a regularity statement which goes a long way towards identifying the dual domain of $\mathcal{H}_{A}$ with its domain (thus establishing self-adjointness). The second part is an estimate for the Hessian on pairs $(\alpha, \varphi)$ that do not necessarily satisfy the boundary conditions. This degree of generality is necessary since the Lagrangian boundary conditions are nonlinear, so differences in $\mathcal{A}(Y, \mathcal{L})$ or derivatives of tangent vectors only satisfy the boundary conditions up to some small curvature term.

Lemma 3.5 The following holds for every $p>1$ and every $A \in \mathcal{A}(Y, \mathcal{L})$.

(i) If $(\alpha, \varphi) \in L^{p}\left(Y, \mathrm{~T}^{*} Y \otimes \mathfrak{g}\right) \times L^{p}(Y, \mathfrak{g})$ and there is a constant $c$ such that

$$
\left|\int_{Y}\left\langle\alpha,\left(* \mathrm{~d}_{A} \beta-\mathrm{d}_{A} \psi\right)\right\rangle-\int_{Y}\left\langle\varphi, \mathrm{d}_{A}^{*} \beta\right\rangle\right| \leq c\|(\beta, \psi)\|_{L^{p^{*}}(Y)}
$$


for every $(\beta, \psi) \in \Omega^{1}(Y, \mathfrak{g}) \times \Omega^{0}(Y, \mathfrak{g})$ with $\left.\beta\right|_{\partial Y} \in \mathrm{d}_{\left.A\right|_{\Sigma}} \Omega^{0}(\Sigma, \mathfrak{g})$ and $\left.* \beta\right|_{\partial Y}=$ 0 , then $(\alpha, \varphi) \in W^{1, p}\left(Y, \mathrm{~T}^{*} Y \otimes \mathfrak{g}\right) \times W^{1, p}(Y, \mathfrak{g})$ and it satisfies $\left.* \alpha\right|_{\partial Y}=0$ and $\mathrm{d}_{\left.A\right|_{\partial Y}}\left(\left.\alpha\right|_{\partial Y}\right)=0$ in the weak sense.

(ii) There is a constant $C$ such that

$$
\begin{aligned}
\|(\alpha, \varphi)\|_{W^{1, p}(Y)} \leq C( & \left\|\mathrm{d}_{A} \alpha-\mathrm{d}_{A} \varphi\right\|_{L^{p}(Y)}+\left\|\mathrm{d}_{A}^{*} \alpha\right\|_{L^{p}(Y)}+\|(\alpha, \varphi)\|_{L^{p}(Y)} \\
& \left.+\left\|\left.* \alpha\right|_{\Sigma}\right\|_{b W^{1-1 / p, p}(\Sigma)}+\left\|\mathrm{d}_{\left.A\right|_{\Sigma}}\left(\left.\alpha\right|_{\Sigma}\right)\right\|_{b W^{-1 / p, p}(\Sigma)}\right)
\end{aligned}
$$

for all $\alpha \in \Omega^{1}(Y, \mathfrak{g})$ and $\varphi \in \Omega^{0}(Y, \mathfrak{g})$.

Before we prove this lemma let us draw a conclusion that will be useful for the exponential decay analysis.

Corollary 3.6 Let $p>1$ and $A \in \mathcal{A}(Y, \mathcal{L})$ be a nondegenerate critical point of $\mathcal{C S}_{\mathcal{L}}+h_{f}$. Then there is a constant $C$ such that

$$
\begin{aligned}
\|\alpha\|_{W^{1, p}(Y)} \leq C\left(\left\|\mathrm{~d}_{A} \alpha+\mathrm{d} X_{f}(A) \alpha\right\|_{L^{p}(Y)}+\left\|\mathrm{d}_{A}^{*} \alpha\right\|_{L^{p}(Y)}\right. \\
\left.+\left\|\left.* \alpha\right|_{\Sigma}\right\|_{b W^{1-1 / p, p}(\Sigma)}+\left\|\Pi_{A}^{\perp}\left(\left.\alpha\right|_{\Sigma}\right)\right\|_{L^{p}(\Sigma)}\right)
\end{aligned}
$$

for every $\alpha \in \Omega^{1}(Y, \mathfrak{g})$, where $\Pi_{A}^{\perp}: \Omega^{1}(\Sigma, \mathfrak{g}) \rightarrow T_{A} \mathcal{L}^{\perp}$ denotes the $L^{2}$ orthogonal projection onto the $L^{2}$ orthogonal complement of $\mathrm{T}_{A} \mathcal{L}$.

Proof By Lemma 3.5 (ii) with $\varphi=0$ we have

$$
\begin{aligned}
\|\alpha\|_{W^{1, p}(Y)} \leq C\left(\left\|\mathrm{~d}_{A} \alpha\right\|_{L^{p}(Y)}+\left\|\mathrm{d}_{A}^{*} \alpha\right\|_{L^{p}(Y)}+\|\alpha\|_{L^{p}(Y)}\right. \\
\left.+\left\|\left.* \alpha\right|_{\Sigma}\right\|_{b W^{1-1 / p, p}(\Sigma)}+\left\|\mathrm{d}_{\left.A\right|_{\Sigma}}\left(\left.\alpha\right|_{\Sigma}\right)\right\|_{b W^{-1 / p, p}(\Sigma)}\right) \\
\leq C^{\prime}\left(\left\|\mathrm{d}_{A^{\alpha}} \alpha+\mathrm{d} X_{f}(A) \alpha\right\|_{L^{p}(Y)}+\left\|\mathrm{d}_{A}^{*} \alpha\right\|_{L^{p}(Y)}\right. \\
\left.\quad+\left\|\left.* \alpha\right|_{\Sigma}\right\|_{b W^{1-1 / p, p}(\Sigma)}+\left\|\Pi_{A}^{\perp}\left(\left.\alpha\right|_{\Sigma}\right)\right\|_{L^{p}(\Sigma)}+\|\alpha\|_{L^{p}(Y)}\right) .
\end{aligned}
$$

Here we have used the estimate $\left\|\mathrm{d} X_{f}(A) \alpha\right\|_{L^{p}(Y)} \leq c\|\alpha\|_{L^{p}(Y)}$ of Proposition D.1 (iv) and Lemma 3.4. We added the term $\left\|\Pi_{A}^{\perp}\left(\left.\alpha\right|_{\Sigma}\right)\right\|_{L^{p}(\Sigma)}$ on the right since

$$
\Pi_{A}^{\perp}\left(\left.\alpha\right|_{\Sigma}\right)=\left.0 \quad \Longleftrightarrow \quad \alpha\right|_{\Sigma} \in \mathrm{T}_{A} \mathcal{L}
$$

and the restriction of the operator $\mathcal{H}_{A}$ to the subspace $\{(\alpha, 0)\} \subset \operatorname{dom} \mathcal{H}_{A}$ is injective. Hence the operator $\alpha \mapsto\left(\mathrm{d}_{A} \alpha+\mathrm{d} X_{f}(A) \alpha, \mathrm{d}_{A}^{*} \alpha,\left.* \alpha\right|_{\Sigma}, \Pi_{A}^{\perp}\left(\left.\alpha\right|_{\Sigma}\right)\right)$ is injective and it follows that the compact term $\|\alpha\|_{L^{p}(Y)}$ on the right can be dropped. This proves the corollary. 
Proof of Lemma 3.5 It suffices to prove the lemma in the case $\left.* A\right|_{\partial Y}=0$. The general case can be reduced to this by a compact perturbation of the operator (leaving the boundary conditions fixed). To prove (i) consider a pair $(\alpha, \varphi) \in L^{p}\left(Y, \mathrm{~T}^{*} Y \otimes \mathfrak{g}\right) \times$ $L^{p}(Y, \mathfrak{g})$ that satisfies (20) with a constant $c$. Let $\zeta \in \Omega^{0}(Y, \mathfrak{g})$ with $\left.\frac{\partial \zeta}{\partial \nu}\right|_{\partial Y}=0$ and choose $(\beta, \psi)=\left(\mathrm{d}_{A} \zeta, 0\right)$. Then $\left.* \beta\right|_{\partial Y}=0$ and $\left.\beta\right|_{\partial Y}=\mathrm{d}_{\left.A\right|_{\Sigma}}\left(\left.\zeta\right|_{\Sigma}\right)$ and hence, by (20),

$$
\begin{aligned}
\left|\int_{Y}\left\langle\varphi, \Delta_{A} \zeta\right\rangle\right| & \leq c\left\|\mathrm{~d}_{A} \zeta\right\|_{L^{p^{*}}(Y)}+\left|\int_{Y}\left\langle\alpha, *\left[F_{A}, \zeta\right]\right\rangle\right| \\
& \leq\left(c+c\|A\|_{L^{\infty}(Y)}+\left\|F_{A}\right\|_{L^{\infty}(Y)}\|\alpha\|_{L^{p}(Y)}\right)\|\zeta\|_{W^{1, p^{*}}(Y)}
\end{aligned}
$$

Hence it follows from the regularity theory for the Neumann problem (see Agmon, Douglis and Nirenberg [1] or eg Wehrheim [34, Theorem 2.3']) that $\varphi \in W^{1, p}(Y, \mathfrak{g})$ and

$$
\|\varphi\|_{W^{1, p}(Y)} \leq C\left(c+\|(\alpha, \varphi)\|_{L^{p}(Y)}\right),
$$

for a suitable constant $C=C(A)$.

Now fix a vector field $Z \in \operatorname{Vect}(Y)$ with $\|Z\|_{L^{\infty}(Y)} \leq 1$ that is perpendicular to $\partial Y$. Then it follows from (20) with $\beta=0$ and $\psi=\mathcal{L}_{Z} \zeta$ that

$$
\begin{aligned}
\left|\int_{Y}\left\langle\alpha, \mathrm{d}\left(\mathcal{L}_{Z} \zeta\right)\right\rangle\right| & \leq c\left\|\mathcal{L}_{Z} \zeta\right\|_{L^{p^{*}}(Y)}+\left|\int_{Y}\left\langle\alpha,\left[A, \mathcal{L}_{Z} \zeta\right]\right\rangle\right| \\
& \leq\left(c+\|A\|_{L^{\infty}(Y)}\|\alpha\|_{L^{p}(Y)}\right)\|\zeta\|_{W^{1, p^{*}}(Y)}
\end{aligned}
$$

for every $\zeta \in \Omega^{0}(Y, \mathfrak{g})$. Choosing $\psi=0$ and $\beta=*\left(\iota_{Z} g \wedge \mathrm{d} \zeta\right)$ gives

$$
\begin{aligned}
&\left|\int_{Y}\left\langle\alpha, \mathrm{d}^{*}\left(\iota_{Z} g \wedge \mathrm{d} \zeta\right)\right\rangle\right| \\
& \leq c\|\iota Z g \wedge \mathrm{d} \zeta\|_{L^{p^{*}(Y)}}+\left|\int_{Y}\left\langle\varphi, \mathrm{d}_{A}(\iota Z g \wedge \mathrm{d} \zeta)\right\rangle\right|+\left|\int_{Y}\langle\alpha, *[A \wedge *(\iota Z g \wedge \mathrm{d} \zeta)]\rangle\right| \\
&(24) \leq\left(c+C_{Z}\|\varphi\|_{L^{p}(Y)}+\|A\|_{L^{\infty}(Y)}\|(\alpha, \varphi)\|_{L^{p}(Y)}\right)\|\zeta\|_{W^{1, p^{*}}(Y)}
\end{aligned}
$$

for every $\zeta \in \Omega^{0}(Y, \mathfrak{g})$ with $\left.\zeta\right|_{\partial Y}=0$, where $C_{Z}:=\left\|\mathrm{d} \iota_{Z} g\right\|_{L^{\infty}(Y)}$. Here we have used (20) with $\left.* \beta\right|_{\partial Y}=0$ and $\left.\beta\right|_{\partial Y}=0$. Combining (23) and (24) we obtain the estimate

$$
\left|\int_{Y}\langle\alpha(Z), \Delta \zeta\rangle\right| \leq\left(2 c+C^{\prime}\|(\alpha, \varphi)\|_{L^{p}(Y)}\right)\|\zeta\|_{W^{1, p^{*}}(Y)}
$$

for every $\zeta \in \Omega^{0}(Y, \mathfrak{g})$ with $\left.\zeta\right|_{\partial Y}=0$ and a suitable constant $C^{\prime}=C^{\prime}(A, Z)$ (see [34, Theorem 5.3 (ii)]). This implies $\alpha(Z) \in W^{1, p}(Y, \mathfrak{g})$ and

$$
\|\alpha(Z)\|_{W^{1, p}(Y)} \leq C\left(c+\|(\alpha, \varphi)\|_{L^{p}(Y)}\right),
$$


where the constant $C$ depends on $A$ and the vector field $Z$. This proves the interior regularity of $\alpha$ as well as the regularity of its normal component. Moreover, partial integration now shows that, for every $\zeta \in \Omega^{0}(Y, \mathfrak{g})$ with $\left.\zeta\right|_{\partial Y}=0$, we have

$$
\left|\int_{\partial Y}\left\langle\alpha(Z), \frac{\partial \zeta}{\partial \nu}\right\rangle\right| \leq\left(2 c+C^{\prime}\|(\alpha, \varphi)\|_{L^{p}(Y)}+\|\mathrm{d}(\alpha(Z))\|_{L^{p}(Y)}\right)\|\zeta\|_{W^{1, p^{*}}(Y)} .
$$

In particular, we can fix any normal derivative $\frac{\partial \zeta}{\partial v}=g \in \Omega^{0}(\partial Y, \mathfrak{g})$ and find an admissible function $\zeta \in \Omega^{0}(Y, \mathfrak{g})$ with $\left.\zeta\right|_{\partial Y}=0$ and $\|\zeta\|_{W^{1, p^{*}}(Y)}$ arbitrarily small. Thus we have $\int_{\partial Y}\langle\alpha(Z), g\rangle=0$ for all $g \in \Omega^{0}(\partial Y, \mathfrak{g})$, and hence $\alpha(Z)=0$ for normal vector fields $Z$, ie $\left.* \alpha\right|_{\partial Y}=0$.

To deal with the tangential components near the boundary $\partial Y=\Sigma$ we use normal geodesics to identify a neighbourhood of the boundary with $[0, \varepsilon) \times \Sigma$ with the split metric $\mathrm{d} t^{2}+g_{t}$, where $\left(g_{t}\right)_{t \in[0, \varepsilon)}$ is a smooth family of metrics on $\Sigma$. In this splitting we write

$$
\alpha=\alpha_{\Sigma}+a \mathrm{~d} t
$$

for $\alpha_{\Sigma} \in L^{p}\left([0, \varepsilon) \times \Sigma, \mathrm{T}^{*} \Sigma \otimes \mathfrak{g}\right)$ and $a \in W^{1, p}([0, \varepsilon) \times \Sigma, \mathfrak{g})$. Then

$$
\left.a\right|_{t=0}=0, \quad\|a\|_{W^{1, p}} \leq C\left(c+\|(\alpha, \varphi)\|_{L^{p}(Y)}\right)
$$

by (25). From now on $*, \mathrm{~d}$, and $\mathrm{d}^{*}$ will denote the Hodge operator, the exterior derivative, and its adjoint on $\Sigma$. We abbreviate $I:=[0, \varepsilon)$ and denote by $\mathcal{C}_{0}^{\infty}(I \times \Sigma)$ the space of functions with compact support in $(0, \varepsilon) \times \Sigma$. Then the inequality $(20)$ can be rewritten as

$$
\begin{aligned}
& \mid \int_{I \times \Sigma}\left\langle\alpha_{\Sigma},\left(* \partial_{t} \beta_{\Sigma}-* \mathrm{~d} b+\mathrm{d} \psi\right)\right\rangle \\
& \quad-\int_{I \times \Sigma}\left\langle a,\left(\partial_{t} \psi-* \mathrm{~d} \beta_{\Sigma}\right)\right\rangle+\int_{I \times \Sigma}\left\langle\varphi,\left(\partial_{t} b-\mathrm{d}^{*} \beta_{\Sigma}\right)\right\rangle \mid \leq c\left\|\left(\beta_{\Sigma}, b, \psi\right)\right\|_{L^{p^{*}}(I \times \Sigma)}
\end{aligned}
$$

for all $\beta_{\Sigma} \in \mathcal{C}_{0}^{\infty}\left(I \times \Sigma, \mathrm{T}^{*} \Sigma \otimes \mathfrak{g}\right)$ and $b, \psi \in \mathcal{C}_{0}^{\infty}(I \times \Sigma, \mathfrak{g})$. Partial integration in the terms involving $a$ and $\varphi$ then yields

$$
\left|\int_{I \times \Sigma}\left\langle\alpha_{\Sigma},\left(\partial_{t} \beta_{\Sigma}-\mathrm{d} b-* \mathrm{~d} \psi\right)\right\rangle\right| \leq\left(c+\|a\|_{W^{1, p}}+\|\varphi\|_{W^{1, p}}\right)\left\|\left(\beta_{\Sigma}, b, \psi\right)\right\|_{L^{p^{*}}} .
$$

Since $\mathcal{C}_{0}^{\infty}(I \times \Sigma)$ is dense in $L^{p^{*}}(I \times \Sigma)$ we obtain $\partial_{t} \alpha_{\Sigma} \in L^{p}\left(I \times \Sigma, \mathrm{T}^{*} \Sigma \otimes \mathfrak{g}\right)$ and $* \mathrm{~d} \alpha_{\Sigma}, \mathrm{d}^{*} \alpha_{\Sigma} \in L^{p}(I \times \Sigma, \mathfrak{g})$ with corresponding estimates. Hence $\nabla_{\Sigma} \alpha_{\Sigma}$ is of class $L^{p}$ (see eg [35, Lemma 2.9]); so $\alpha_{\Sigma}$ is of class $W^{1, p}$ and satisfies the estimate

$$
\left\|\alpha_{\Sigma}\right\|_{W^{1, p}} \leq C\left(c+\|a\|_{W^{1, p}}+\|\varphi\|_{W^{1, p}}+\left\|\alpha_{\Sigma}\right\|_{L^{p}}\right)
$$


with yet another constant $C$. In combination with (22) and (25) this proves the regularity claimed in (i) and the estimate

$$
\|(\alpha, \varphi)\|_{W^{1, p}(Y)} \leq C\left(c+\|(\alpha, \varphi)\|_{L^{p}(Y)}\right) .
$$

To prove the second boundary condition on $\left.\alpha\right|_{\partial Y}$ we use partial integration in (20) to obtain

$$
\left|\int_{\Sigma}\langle\alpha \wedge \beta\rangle\right| \leq\left(c+\left\|\mathrm{d}_{A} \alpha\right\|_{L^{p}(Y)}+\left\|\mathrm{d}_{A} \varphi\right\|_{L^{p}(Y)}\right)\|\beta\|_{L^{p^{*}}(Y)}
$$

for every $\beta \in \Omega^{1}(Y, \mathfrak{g})$ with $\left.* \beta\right|_{\Sigma}=0$ and $\left.\beta\right|_{\Sigma} \in \mathrm{d}_{\left.A\right|_{\Sigma}} \Omega^{0}(\Sigma, \mathfrak{g})$. In particular, we can fix $\left.\beta\right|_{\Sigma}=\mathrm{d}_{\left.A\right|_{\Sigma}} \xi$ for any $\xi \in \mathcal{C}^{\infty}(\Sigma, \mathfrak{g})$ and find admissible $\beta \in \Omega^{1}(Y, \mathfrak{g})$ with $\left.* \beta\right|_{\Sigma}=0$ and $\|\beta\|_{L^{p^{*}}(Y)}$ arbitrarily small. Thus we have $\int_{\Sigma}\left\langle\alpha \wedge \mathrm{d}_{\left.A\right|_{\Sigma}} \xi\right\rangle=0$ for all $\xi \in \Omega^{0}(\Sigma, \mathfrak{g})$, that is $\mathrm{d}_{\left.A\right|_{\Sigma}}\left(\left.\alpha\right|_{\Sigma}\right)=0$ in the weak sense. This proves (i).

To prove (ii) let $(\alpha, \varphi) \in \Omega^{1}(Y, \mathfrak{g}) \times \Omega^{0}(Y, \mathfrak{g})$ be given and choose $\gamma \in \Omega^{1}(Y, \mathfrak{g})$ such that

$$
\left.* \gamma\right|_{\Sigma}=\left.* \alpha\right|_{\Sigma},\left.\quad \gamma\right|_{\Sigma}=0, \quad\|\gamma\|_{W^{1, p}(Y)} \leq 2\left\|\left.* \alpha\right|_{\Sigma}\right\|_{b W^{1-1 / p, p}(\Sigma)},
$$

and denote $\alpha^{\prime}:=\alpha-\gamma$. There is a constant $C_{0}=C_{0}(A)>0$ such that $\left\|\mathcal{H}_{A}(\gamma, 0)\right\|_{L^{p}(Y)} \leq$ $C_{0}\left\|\left.* \alpha\right|_{\Sigma}\right\|_{b W^{1-1 / p, p}(\Sigma)}$ and hence

$$
\left\|\mathcal{H}_{A}\left(\alpha^{\prime}, \varphi\right)\right\|_{L^{p}(Y)} \leq\left\|\mathcal{H}_{A}(\alpha, \varphi)\right\|_{L^{p}(Y)}+C_{0}\left\|\left.* \alpha\right|_{\Sigma}\right\|_{b W^{1-1 / p, p}(\Sigma)}=: c .
$$

Then it follows from (18) that, for every pair $(\beta, \psi) \in \Omega^{1}(Y, \mathfrak{g}) \times \Omega^{0}(Y, \mathfrak{g})$ with $\left.* \beta\right|_{\Sigma}=0$, we have

$$
\left|\left\langle\left(\alpha^{\prime}, \varphi\right), \mathcal{H}_{A}(\beta, \psi)\right\rangle\right| \leq c\|(\beta, \psi)\|_{L^{p^{*}}(Y)}+\left|\int_{\Sigma}\langle\alpha \wedge \beta\rangle\right|
$$

Let $\zeta \in \Omega^{0}(Y, \mathfrak{g})$ with $\left.\frac{\partial \zeta}{\partial \nu}\right|_{\partial Y}=0$ and choose $(\beta, \psi)=\left(\mathrm{d}_{A} \zeta, 0\right)$. Then, by Lemma 3.4, we have

$$
\left|\int_{\Sigma}\left\langle\alpha \wedge \mathrm{d}_{A} \zeta\right\rangle\right| \leq\left\|\mathrm{d}_{\left.A\right|_{\Sigma}}\left(\left.\alpha\right|_{\Sigma}\right)\right\|_{b W^{-1 / p, p}(\Sigma)}\|\zeta\|_{W^{1, p^{*}}(Y)}
$$

Geometry $\&$ Topology, Volume 12 (2008) 
and hence, by (26),

$$
\begin{aligned}
& \left|\int_{Y}\left\langle\varphi, \Delta_{A} \zeta\right\rangle\right|=\left|\left\langle\left(\alpha^{\prime}, \varphi\right), \mathcal{H}_{A}\left(\mathrm{~d}_{A} \zeta, 0\right)\right\rangle-\int_{Y}\left\langle\alpha^{\prime}, *\left[F_{A}, \zeta\right]\right\rangle\right| \\
& \leq c\left\|\mathrm{~d}_{A} \zeta\right\|_{L^{p^{*}}(Y)}+\left|\int_{\Sigma}\left\langle\alpha \wedge \mathrm{d}_{A} \zeta\right\rangle\right|+\left\|\alpha^{\prime}\right\|_{L^{p}(Y)}\left\|F_{A}\right\|_{L^{\infty}(Y)}\|\zeta\|_{L^{p^{*}}(Y)} \\
& \leq C\left(\left\|\mathcal{H}_{A}(\alpha, \varphi)\right\|_{L^{p}(Y)}+\left\|\left.* \alpha\right|_{\Sigma}\right\|_{b W^{1-1 / p, p}(\Sigma)}\right. \\
& \left.\quad+\left\|\mathrm{d}_{\left.A\right|_{\Sigma}}\left(\left.\alpha\right|_{\Sigma}\right)\right\|_{b W^{-1 / p, p}(\Sigma)}+\|\alpha\|_{L^{p}(Y)}\right)\|\zeta\|_{W^{1, p^{*}}(Y)}
\end{aligned}
$$

for a suitable constant $C=C(A)$. (Compare this with (21).) As in the proof of (i) this implies

$$
\begin{aligned}
\|\varphi\|_{W^{1, p}(Y)} \leq C( & \left\|\mathcal{H}_{A}(\alpha, \varphi)\right\|_{L^{p}(Y)}+\left\|\left.* \alpha\right|_{\Sigma}\right\|_{b W^{1-1 / p, p}(\Sigma)} \\
& \left.+\left\|\mathrm{d}_{\left.A\right|_{\Sigma}}\left(\left.\alpha\right|_{\Sigma}\right)\right\|_{b W^{-1 / p, p}(\Sigma)}+\|(\alpha, \varphi)\|_{L^{p}(Y)}\right)
\end{aligned}
$$

with a possibly larger constant $C$. (Compare this with (22).) To prove the same estimate for $\alpha^{\prime}$ (and hence for $\alpha$ ) one can repeat the argument in the proof of (i), because in this part of the argument the inequality (26) is only needed for $(\beta, \psi)$ with $\left.* \beta\right|_{\Sigma}=0$ and $\left.\beta\right|_{\Sigma}=0$. This proves (ii) and the lemma.

Proof of Proposition 3.1 We prove (ii) by induction. Observe that

$$
\left\|\mathrm{d} X_{f}(A) \alpha\right\|_{W^{k, p}(Y)} \leq C\|\alpha\|_{W^{k, p}(Y)}
$$

for all $\alpha \in \Omega^{1}(Y, \mathfrak{g})$ and a constant $C=C(A, f)$, by Proposition D.1 (iv). Hence it suffices to prove the estimate with $f=0$. For $k=0$ regularity holds by assumption and the estimate follows from Lemma 3.5 (ii), using the fact that $\mathrm{d}_{\left.A\right|_{\Sigma}} \Omega^{0}(\Sigma, \mathfrak{g}) \subset \mathrm{T}_{A} \mathcal{L}$, so $\mathrm{d}_{\left.A\right|_{\Sigma}}\left(\left.\alpha\right|_{\Sigma}\right)=0$. (For $p=2$ an elementary proof of the estimate was given at the beginning of the section.) Thus we have proved (ii) for $k=0$. It follows that $\mathcal{H}_{A}$ has a finite dimensional kernel and a closed image.

Now let $k \geq 1$ and suppose that (ii) has been established for $k-1$. Let $(\alpha, \varphi) \in \operatorname{dom} \mathcal{H}_{A}$ and assume that $\mathcal{H}_{A}(\alpha, \varphi)$ is of class $W^{k, p}$. By the induction hypothesis $(\alpha, \varphi)$ is of class $W^{k, p}$ and

$$
\|(\alpha, \varphi)\|_{W^{k, p}(Y)} \leq C\left(\left\|\mathcal{H}_{A}(\alpha, \varphi)\right\|_{W^{k-1, p}(Y)}+\|(\alpha, \varphi)\|_{L^{p}(Y)}\right) .
$$

Let $X_{1}, \ldots, X_{k} \in \operatorname{Vect}(Y)$. Then, using the symmetry of $\mathcal{H}_{A}$ and integration by parts, we obtain for every smooth pair $(\beta, \psi) \in \Omega^{1}(Y) \times \Omega^{0}(Y)$ with compact support in 
the interior of $Y$, we have

$$
\begin{aligned}
\mid\left\langle\mathcal{L}_{X_{1}} \cdots\right. & \left.\mathcal{L}_{X_{k}}(\alpha, \varphi), \mathcal{H}_{A}(\beta, \psi)\right\rangle \mid \\
& =\left|\left\langle(\alpha, \varphi), \mathcal{L}_{X_{k}}^{*} \cdots \mathcal{L}_{X_{1}}^{*} \mathcal{H}_{A}(\beta, \psi)\right\rangle\right| \\
& \leq\left|\left\langle(\alpha, \varphi), \mathcal{H}_{A} \mathcal{L}_{X_{k}}^{*} \cdots \mathcal{L}_{X_{1}}^{*}(\beta, \psi)\right\rangle\right|+C_{1}\|(\alpha, \varphi)\|_{W^{k, p}(Y)}\|(\beta, \psi)\|_{L^{p^{*}}(Y)} \\
& =\left|\left\langle\mathcal{L}_{X_{1}} \cdots \mathcal{L}_{X_{k}} \mathcal{H}_{A}(\alpha, \varphi),(\beta, \psi)\right\rangle\right|+C_{1}\|(\alpha, \varphi)\|_{W^{k, p}(Y)}\|(\beta, \psi)\|_{L^{p^{*}}(Y)} \\
& \leq C_{2}\left(\left\|\mathcal{H}_{A}(\alpha, \varphi)\right\|_{W^{k, p}(Y)}+\|(\alpha, \varphi)\|_{W^{k, p}(Y)}\right)\|(\beta, \psi)\|_{L^{p^{*}}(Y)}
\end{aligned}
$$

with uniform constants $C_{i}$. This estimate extends to the $W^{1, p^{*}}$-closure, so it holds for all $(\beta, \psi)$ with zero boundary conditions. However, in order to apply Lemma 3.5 (i) to the pair $\mathcal{L}_{X_{1}} \cdots \mathcal{L}_{X_{k}}(\alpha, \varphi)$ we would have to allow for more general test functions $(\beta, \psi)$. Unfortunately, this weak equation does not extend directly, but we can still use the arguments of Lemma 3.5. For that purpose let the vector fields $X_{1}, \ldots, X_{k} \in \operatorname{Vect}(Y)$ be tangential to the boundary. Then the boundary condition $\left.* \alpha\right|_{\partial Y}=0$ will be preserved, and the Lie derivatives $\mathcal{L}_{X_{i}}$ in the following all have a dual $\mathcal{L}_{X_{i}}^{*}$ which does not include a boundary term. To adapt the proof of Lemma 3.5 (i) to $\mathcal{L}_{X_{1}} \cdots \mathcal{L}_{X_{k}}(\alpha, \varphi)$ instead of $(\alpha, \varphi)$ we replace (21) and (23), which use test functions with nonzero boundary values.

Instead of (21) we calculate for all $\zeta \in \Omega^{0}(Y, \mathfrak{g})$ with $\left.\frac{\partial \zeta}{\partial \nu}\right|_{\partial Y}=0$ and with a $W^{k, p_{-}}$ approximation $\Omega^{0}(Y, \mathfrak{g}) \ni \varphi_{j} \rightarrow \varphi$

$$
\begin{aligned}
&\left|\left\langle\mathcal{L}_{X_{1}} \ldots \mathcal{L}_{X_{k}} \varphi, \Delta_{A} \zeta\right\rangle\right|=\lim _{j \rightarrow \infty}\left|\left\langle\mathrm{d}_{A} \mathcal{L}_{X_{1}} \ldots \mathcal{L}_{X_{k}} \varphi_{j}, \mathrm{~d}_{A} \zeta\right\rangle\right| \\
& \leq \lim _{j \rightarrow \infty}\left(\left|\left\langle\mathcal{L}_{X_{1}} \ldots \mathcal{L}_{X_{k}} \mathrm{~d}_{A} \varphi_{j}, \mathrm{~d}_{A} \zeta\right\rangle\right|+C_{1}\left\|\varphi_{j}\right\|_{W^{k, p}}\|\zeta\|_{W^{1, p^{*}}}\right) \\
&=\left|\left\langle\mathcal{L}_{X_{2}} \ldots \mathcal{L}_{X_{k}} \mathrm{~d}_{A} \varphi, \mathcal{L}_{X_{1}}^{*} \mathrm{~d}_{A} \zeta\right\rangle\right|+C_{1}\|\varphi\|_{W^{k, p}}\|\zeta\|_{W^{1, p}} \\
& \leq\left|\left\langle\mathcal{L}_{X_{2}} \ldots \mathcal{L}_{X_{k}} * \mathrm{~d}_{A} \alpha, \mathrm{d}_{A} \mathcal{L}_{X_{1}}^{*} \zeta\right\rangle\right|+\left|\left\langle\mathcal{L}_{X_{1}} \ldots \mathcal{L}_{X_{k}}\left(* \mathrm{~d}_{A} \alpha-\mathrm{d}_{A} \varphi\right), \mathrm{d}_{A} \zeta\right\rangle\right| \\
&+C_{2}\|\varphi\|_{W^{k, p}}\|\zeta\|_{W^{1, p^{*}}} \\
& \leq\left|\left\langle * \mathrm{~d}_{A} \alpha, \mathrm{d}_{A} \mathcal{L}_{X_{k}}^{*} \ldots \mathcal{L}_{X_{1}}^{*} \zeta\right\rangle\right|+\left|\left\langle * \mathrm{~d}_{A} \alpha,\left[\mathcal{L}_{X_{k}}^{*} \ldots \mathcal{L}_{X_{2}}^{*}, \mathrm{~d}_{A}\right] \mathcal{L}_{X_{1}}^{*} \zeta\right\rangle\right| \\
&+C_{3}\left(\left\|* \mathrm{~d}_{A} \alpha-\mathrm{d}_{A} \varphi\right\|_{W^{k, p}}+\|\varphi\|_{W^{k, p}}\right)\|\zeta\|_{W^{1, p}} \\
& \leq C_{4}\left(\left\|\mathcal{H}_{A}(\alpha, \varphi)\right\|_{W^{k, p}}+\|(\alpha, \varphi)\|_{W^{k, p}}\right)\|\zeta\|_{W^{1, p^{*}}}
\end{aligned}
$$

with uniform constants $C_{i}$. Here the components of $\left[\mathcal{L}_{X_{k}}^{*} \ldots \mathcal{L}_{X_{2}}^{*}, \mathrm{~d}_{A}\right] \mathcal{L}_{X_{1}}^{*} \zeta$ are sums of derivatives of $\zeta$ including at most one normal derivative, so all but one derivative can be moved to the left hand side $* \mathrm{~d}_{A} \alpha$ by partial integration. Moreover, we have 
used the fact that $\left.\mathrm{d}_{A} \mathcal{L}_{X_{k}}^{*} \ldots \mathcal{L}_{X_{1}}^{*} \zeta\right|_{\partial Y} \in \mathrm{T}_{A} \mathcal{L}$ to obtain

$$
\begin{aligned}
\left\langle * \mathrm{~d}_{A} \alpha, \mathrm{d}_{A} \mathcal{L}_{X_{k}}^{*} \ldots \mathcal{L}_{X_{1}}^{*} \zeta\right\rangle & =\left\langle\alpha, *\left[F_{A}, \mathcal{L}_{X_{k}}^{*} \ldots \mathcal{L}_{X_{1}}^{*} \zeta\right\rangle\right. \\
& =\left\langle\mathcal{L}_{X_{1}} \ldots \mathcal{L}_{X_{k}} *\left[F_{A} \wedge \alpha\right], \zeta\right\rangle .
\end{aligned}
$$

The last term can be estimated by $\|\alpha\|_{W^{k, p}}\|\zeta\|_{L^{p^{*}}}$.

Instead of (23) we pick a $W^{k, p}$-approximation $\Omega^{1}(Y, \mathfrak{g}) \ni \alpha_{j} \rightarrow \alpha$ satisfying the boundary condition $\left.* \alpha_{j}\right|_{\partial Y}=0$ and hence $\left.* \mathcal{L}_{X_{1}} \ldots \mathcal{L}_{X_{k}} \alpha_{j}\right|_{\partial Y}=0$. Then we obtain for all $\zeta \in \Omega^{0}(Y, \mathfrak{g})$

$$
\begin{aligned}
\left|\int_{Y}\left\langle\mathcal{L}_{X_{1}} \ldots \mathcal{L}_{X_{k}} \alpha, \mathrm{d}\left(\mathcal{L}_{Z} \zeta\right)\right\rangle\right| \\
\quad \leq \lim _{j \rightarrow \infty}\left(\left|\int_{Y}\left\langle\mathcal{L}_{X_{1}} \ldots \mathcal{L}_{X_{k}} \alpha_{j}, \mathrm{~d}_{A}\left(\mathcal{L}_{Z} \zeta\right)\right\rangle\right|+C_{1}\left\|\alpha_{j}\right\|_{W^{k, p}}\|\zeta\|_{W^{1, p^{*}}}\right) \\
\leq \lim _{j \rightarrow \infty}\left(\left|\int_{Y}\left\langle\mathcal{L}_{X_{1}} \ldots \mathcal{L}_{X_{k}} \mathrm{~d}_{A}^{*} \alpha_{j}, \mathcal{L}_{Z} \zeta\right\rangle\right|+C_{2}\left\|\alpha_{j}\right\|_{W^{k, p}}\|\zeta\|_{W^{1, p^{*}}}\right) \\
\quad=\left|\int_{Y}\left\langle\mathcal{L}_{X_{2}} \ldots \mathcal{L}_{X_{k}} \mathrm{~d}_{A}^{*} \alpha, \mathcal{L}_{X_{1}}^{*} \mathcal{L}_{Z} \zeta\right\rangle\right|+C_{2}\|\alpha\|_{W^{k, p}}\|\zeta\|_{W^{1, p^{*}}} \\
\leq\left\|\mathcal{L}_{X_{1}} \ldots \mathcal{L}_{X_{k}} \mathrm{~d}_{A}^{*} \alpha\right\|_{L^{p}}\left\|\mathcal{L}_{Z} \zeta\right\|_{L^{p^{*}}}+C_{2}\|\alpha\|_{W^{k, p}}\|\zeta\|_{W^{1, p^{*}}} \\
\leq C_{3}\left(\left\|\mathcal{H}_{A}(\alpha, \varphi)\right\|_{W^{k, p}}+\|(\alpha, \varphi)\|_{W^{k, p}}\right)\|\zeta\|_{W^{1, p^{*}}(Y)}
\end{aligned}
$$

with uniform constants $C_{i}$. Now the remaining arguments of Lemma 3.5 (i) go through to prove the regularity $\mathcal{L}_{X_{1}} \ldots \mathcal{L}_{X_{k}}(\alpha, \varphi) \in W^{1, p}$ and the estimate

$$
\left\|\mathcal{L}_{X_{1}} \ldots \mathcal{L}_{X_{k}}(\alpha, \varphi)\right\|_{W^{1, p}} \leq C\left(\left\|\mathcal{H}_{A}(\alpha, \varphi)\right\|_{W^{k, p}}+\|(\alpha, \varphi)\|_{W^{k, p}}\right)
$$

for the tangential derivatives and in the interior. To control the normal derivatives near the boundary we use the same splitting as in Lemma 3.5 (i). If $\mathcal{H}_{A}(\alpha, \varphi) \in W^{k, p}$ then this argument shows that

$$
\begin{aligned}
\partial_{t} \alpha_{\Sigma} & \in \mathrm{d} a-* \mathrm{~d} \varphi+W^{k, p}\left(I \times \Sigma, \mathrm{T}^{*} \Sigma \otimes \mathfrak{g}\right), \\
\partial_{t} a & \in \mathrm{d}^{*} \alpha_{\Sigma}+W^{k, p}(I \times \Sigma, \mathfrak{g}), \\
\partial_{t} \varphi & \in * \mathrm{~d} \beta_{\Sigma}+W^{k, p}(I \times \Sigma, \mathfrak{g}) .
\end{aligned}
$$

This can be used iteratively to replace the derivatives in (28) by normal derivatives. It then follows from the assumption $\mathcal{H}_{A}(\alpha, \varphi) \in W^{k, p}$ and the induction hypothesis $(\alpha, \varphi) \in W^{k, p}$ that $(\alpha, \varphi) \in W^{k+1, p}$ and

$$
\|(\alpha, \varphi)\|_{W^{k+1, p}} \leq C\left(\left\|\mathcal{H}_{A}(\alpha, \varphi)\right\|_{W^{k, p}}+\|(\alpha, \varphi)\|_{L^{p}}\right) .
$$

This finishes the proof of (ii). 
We prove (iii). If $F_{A}+X_{f}(A)=0$ and $(\alpha, \varphi) \in \operatorname{ker} \mathcal{H}_{A}$, then the pair $(\alpha, \varphi)$ is smooth by (ii). Integration by parts shows that $* \mathrm{~d}_{A} \alpha+* \mathrm{~d} X_{f}(A) \alpha$ is orthogonal to $\mathrm{d}_{A} \varphi$, hence both vanish, so the kernel has the required form.

To prove (i) we first show that the cokernel of $\mathcal{H}_{A}$ agrees with its kernel. Let $(\alpha, \varphi) \in$ $L^{2}\left(Y, \mathrm{~T}^{*} Y\right) \times L^{2}(Y)$ be orthogonal to the image of $\mathcal{H}_{A}$. Denote by $\mathcal{H}$ the operator of Lemma 3.5 for the perturbation $f=0$. Then

$$
\langle(\alpha, \varphi), \mathcal{H}(\beta, \psi)\rangle_{L^{2}}=-\left\langle\alpha, * \mathrm{~d} X_{f}(A) \beta\right\rangle_{L^{2}} \leq c\|(\beta, \psi)\|_{L^{2}}
$$

for some constant $c$ and every pair $(\beta, \psi) \in \Omega^{1}(Y, \mathfrak{g}) \times \Omega^{0}(Y, \mathfrak{g})$ satisfying the boundary conditions $\left.* \beta\right|_{\partial Y}=0$ and $\left.\beta\right|_{\partial Y} \in \mathrm{T}_{A} \mathcal{L}$. Hence it follows from Lemma 3.5 (i) that $\alpha \in W^{1,2}\left(Y, \mathrm{~T}^{*} Y\right)$ and $\varphi \in W^{1,2}(Y)$. So by (18)

$$
\begin{aligned}
0= & \int_{Y}\left\langle\left(\mathrm{~d}_{A} \alpha+\mathrm{d} X_{f}(A) \alpha-* \mathrm{~d}_{A} \varphi\right) \wedge \beta\right\rangle+\int_{Y}\left\langle\left(\mathrm{~d}_{A} * \alpha\right) \wedge \psi\right\rangle \\
& -\int_{\partial Y}\langle\alpha \wedge \beta\rangle+\int_{\partial Y}\langle\varphi, * \beta\rangle-\int_{\partial Y}\langle * \alpha, \psi\rangle
\end{aligned}
$$

for all $\beta \in \Omega_{A}^{1}(Y, \mathfrak{g})$ and $\psi \in \Omega^{0}(Y, \mathfrak{g})$. (See (19) for the definition of $\Omega_{A}^{1}(Y, \mathfrak{g})$.) Taking $\left.* \beta\right|_{\partial Y}=0,\left.\beta\right|_{\partial Y}=0$, and $\left.\psi\right|_{\partial Y}=0$ this implies

$$
* \mathrm{~d}_{A} \alpha+* \mathrm{~d} X_{f}(A) \alpha-\mathrm{d}_{A} \varphi=0, \quad \mathrm{~d}_{A}^{*} \alpha=0 .
$$

Taking $(\beta, \psi) \in \operatorname{dom} \mathcal{H}_{A}$ we then get

$$
\int_{\partial Y}\langle\alpha \wedge \beta\rangle+\int_{\partial Y}\langle * \alpha, \psi\rangle=0
$$

for every $\beta \in \Omega_{A}^{1}(Y, \mathfrak{g})$ and every $\psi \in \Omega^{0}(Y, \mathfrak{g})$. This (re-)proves $\left.* \alpha\right|_{\partial Y}=0$ and, since $\left.\beta\right|_{\partial Y}$ can take any value in the Lagrangian subspace $\mathrm{T}_{A} \mathcal{L}$, it also shows that $\left.\alpha\right|_{\partial Y} \in \mathrm{T}_{A} \mathcal{L}$. Thus we have identified the cokernel of $\mathcal{H}_{A}$ with its kernel. Since the kernel is finite dimensional, this proves that $\mathcal{H}_{A}$ is a Fredholm operator. Furthermore, every symmetric Fredholm operator with this property is self-adjoint. (Let $x \in \operatorname{dom} \mathcal{H}^{*}$, ie $\langle x, \mathcal{H} y\rangle=\langle z, y\rangle$ for all $y \in \operatorname{dom} \mathcal{H}$ and some $z$ in the target space. By assumption we can write $z=z_{0}+\mathcal{H} x_{1}$ with $z_{0} \in(\operatorname{im} \mathcal{H})^{\perp}$ and $x_{1} \in \operatorname{dom} \mathcal{H}$. Then, using symmetry, we have $\left\langle x-x_{1}, \mathcal{H} y\right\rangle=\left\langle z_{0}, y\right\rangle=0$ for all $y \in \operatorname{im} \mathcal{H} \cap \operatorname{dom} \mathcal{H}$. The latter is a complement of $\operatorname{ker} \mathcal{H} \subset \operatorname{dom} \mathcal{H}$ so we obtain $x-x_{1} \in(\operatorname{im} \mathcal{H})^{\perp}=\operatorname{ker} \mathcal{H} \subset \operatorname{dom} \mathcal{H}$ and hence $x \in \operatorname{dom} \mathcal{H}$.) This proves the proposition.

\section{The set of critical points}

Using the properties of the Hessian we can now show finiteness of the set of gauge equivalence classes of critical points of the Chern-Simons functional, where the critical 
points are assumed to be nondegenerate. More generally, we establish a compactness result that will be needed to achieve nondegeneracy by a transversality construction.

Proposition 3.7 Fix a Lagrangian submanifold $\mathcal{L} \subset \mathcal{A}(\Sigma)$ that satisfies (L1) and an integer $k \geq 1$. Let $f^{v}$ be a sequence of perturbations converging to $f$ in the $\mathcal{C}^{k+1}$ topology and $A^{v} \in \mathcal{A}(Y, \mathcal{L})$ be a sequence of critical points of $\mathcal{C} \mathcal{S}_{\mathcal{L}}+h_{f^{v}}$. Then there is a sequence of gauge transformations $u^{v} \in \mathcal{G}(Y)$ such that $\left(u^{v}\right)^{*} A^{v}$ has a $\mathcal{C}^{k}$ convergent subsequence.

Moreover, if all the critical points of $\mathcal{C S}_{\mathcal{L}}+h_{f}$ are nondegenerate, then $\mathcal{R}_{f}$ is a finite set.

Proof Fix a constant $p>4$. The critical points of $\mathcal{C} \mathcal{S}_{\mathcal{L}}+h_{f^{v}}$ are $S^{1}$-invariant solutions of the perturbed anti-self-duality equation on $S^{1} \times Y$ and, by Proposition D.1 (iii), they satisfy a uniform $L^{\infty}$ bound on the curvature. Hence, by Uhlenbeck's weak compactness theorem (see Uhlenbeck [32] or Wehrheim [34, Theorem A]), there is a sequence of gauge transformations $u^{v} \in \mathcal{G}(Y)$ such that $\left(u^{v}\right)^{*} A^{v}$ is bounded in $W^{1, p}$. Passing to a subsequence, we may assume that $\left(u^{v}\right)^{*} A^{v}$ converges strongly in $\mathcal{C}^{0}$ and weakly in $W^{1, p}$ to a connection $A \in \mathcal{A}^{1, p}(Y, \mathcal{L})$. The limit connection is a (weak) solution of $F_{A}+X_{f}(A)=0$ and hence, by [35, Theorem A], is gauge equivalent to a smooth solution. Applying a further sequence of gauge transformation we may assume that $A$ is smooth and, by the local slice theorem (eg [34, Theorem F]), that

$$
\mathrm{d}_{A}^{*}\left(\left(u^{v}\right)^{*} A^{v}-A\right)=0,\left.\quad *\left(\left(u^{v}\right)^{*} A^{v}-A\right)\right|_{\partial Y}=0 .
$$

It now follows by induction that $\left(u^{v}\right)^{*} A^{v}$ is uniformly bounded in $W^{k+1, p}$. Namely, if $\left(u^{v}\right)^{*} A^{v}$ is uniformly bounded in $W^{j, p}$ for any $j \in\{1, \ldots, k\}$ then the curvature $F_{\left(u^{v}\right)^{*} A^{v}}=-X_{f^{v}}\left(\left(u^{v}\right)^{*} A^{v}\right)$ is uniformly bounded in $W^{j, p}$, by Proposition D.1 (iii), and hence $\left(u^{v}\right)^{*} A^{v}$ is uniformly bounded in $W^{j+1, p}$ by [35, Theorem 2.6]. Since the Sobolev embedding $W^{k+1, p} \hookrightarrow \mathcal{C}^{k}$ is compact, the sequence $\left(u^{v}\right)^{*} A^{v}$ must have a $\mathcal{C}^{k}$ convergent subsequence.

To prove finiteness in the nondegenerate case it remains to show that nondegenerate critical points are isolated in the quotient $\mathcal{A}(Y, \mathcal{L}) / \mathcal{G}(Y)$. Thus let $A$ be a nondegenerate critical point and $A^{v} \in \mathcal{A}(Y, \mathcal{L})$ be a sequence of critical points converging to $A$ in the $W^{1, p}$ topology (for some $p>2$ ). Then, by the local slice theorem, there exists a sequence of gauge transformations $u^{v} \in \mathcal{G}(Y)$, converging to $\mathbb{1}$ in the $W^{2, p}$ topology, such that $\left(u^{v}\right)^{*} A^{v}$ satisfies $(29)$. Since $\mathcal{A}^{1, p}(Y, \mathcal{L})$ is a gauge invariant Banach submanifold of $\mathcal{A}^{1, p}(Y)$ it follows that the intersection with a local slice gives 
rise to a Banach submanifold

$$
\mathcal{X}_{A}:=\left\{\begin{array}{l|l}
\alpha \in W^{1, p}\left(Y, \mathrm{~T}^{*} Y \otimes \mathfrak{g}\right) \mid \begin{array}{c}
\left.* \alpha\right|_{\Sigma}=0, \mathrm{~d}_{A}^{*} \alpha=0,\|\alpha\|_{W^{1, p}}<\varepsilon \\
A+\alpha \in \mathcal{A}^{1, p}(Y, \mathcal{L})
\end{array}
\end{array}\right\}
$$

for $\varepsilon>0$ sufficiently small. The tangent space of $\mathcal{X}_{A}$ at $A$ is

$$
\mathrm{T}_{A} \mathcal{X}_{A}=\left\{\alpha \in W^{1, p}\left(Y, \mathrm{~T}^{*} Y \otimes \mathfrak{g}\right)|* \alpha|_{\Sigma}=0,\left.\alpha\right|_{\Sigma} \in \mathrm{T}_{A} \mathcal{L}, \mathrm{d}_{A}^{*} \alpha=0\right\} .
$$

Define the map $\mathcal{F}_{A}: \mathcal{X}_{A} \times\left\{\varphi \in W^{1, p}(Y, \mathfrak{g}) \mid \varphi \perp \operatorname{ker} \mathrm{d}_{A}\right\} \rightarrow L^{p}\left(Y, \mathrm{~T}^{*} Y \otimes \mathfrak{g}\right)$ by

$$
\mathcal{F}_{A}(\alpha, \varphi):=*\left(F_{A+\alpha}+X_{f}(A+\alpha)\right)-\mathrm{d}_{A} \varphi .
$$

It has a zero at the origin, and we claim that its differential

$$
\mathrm{d} \mathcal{F}_{A}(0,0)(\hat{\alpha}, \hat{\varphi})=*\left(\mathrm{~d}_{A} \hat{\alpha}+\mathrm{d} X_{f}(A) \hat{\alpha}\right)-\mathrm{d}_{A} \hat{\varphi}
$$

is bijective. The injectivity follows from the nondegeneracy of $A$ and the fact that $\operatorname{imd}_{A} \perp \operatorname{im} *\left(\mathrm{~d}_{A}+\mathrm{d} X_{f}(A)\right)$. To check the surjectivity notice that $\mathrm{d} \mathcal{F}_{A}(0,0)$ is the first factor of the Hessian $\mathcal{H}_{A}$. The Hessian is self-adjoint by Proposition 3.1 with cokernel $\left(\operatorname{im} \mathcal{H}_{A}\right)^{\perp}=\operatorname{ker} \mathcal{H}_{A}=H_{A, f}^{1} \times H_{A}^{0}$, so the cokernel of d $\mathcal{F}_{A}(0,0)$ is $H_{A, f}^{1}$, which vanishes by the nondegeneracy assumption. This proves that $\mathrm{d} \mathcal{F}_{A}(0,0)$ is bijective. Since $\left(u^{v}\right)^{*} A^{v}-A \in \mathcal{X}_{A}$ converges to zero in the $W^{1, p}$ norm and $\mathcal{F}_{A}\left(\left(u^{v}\right)^{*} A^{v}-A, 0\right)=0$ for every $v$, it then follows from the inverse function theorem that $\left(u^{v}\right)^{*} A^{v}=A$ for $v$ sufficiently large. This proves the proposition.

For nondegenerate critical points (that is, $H_{A, f}^{1}=0$ ) we have the following control on the kernel of the Hessian, $H_{A}^{0}=\operatorname{kerd}_{A} \subset \Omega^{0}(Y, \mathfrak{g})$, which measures reducibility.

Remark 3.8 The twisted cohomology groups $H_{A}^{0}$ form a vector bundle over the space of pairs $(f, A)$ with $A$ a nondegenerate critical point of $\mathcal{C} \mathcal{S}_{\mathcal{L}}+h_{f}$. In particular, the dimension cannot jump. This follows from the general fact that the cohomology groups $H^{0}$ form a vector bundle over the space of all chain complexes with $H^{1}=0$. To see this consider two chain complexes

$$
C^{0} \stackrel{\mathrm{d}^{0}}{\rightarrow} C^{1} \stackrel{\mathrm{d}^{1}}{\rightarrow} C^{2}, \quad C^{0} \stackrel{\mathrm{d}^{0}+P^{0}}{\longrightarrow} C^{1} \stackrel{\mathrm{d}^{1}+P^{1}}{\longrightarrow} C^{2}
$$

of operators with closed images (between Hilbert spaces) and assume that the first homology of the unperturbed complex vanishes, $H^{1}=\mathrm{kerd}^{1} / \mathrm{im} \mathrm{d}^{0}=0$. (Then the homology of the other complex, $H_{P}^{1}=\operatorname{ker}\left(\mathrm{d}^{1}+P^{1}\right) / \mathrm{im}\left(\mathrm{d}^{0}+P^{0}\right)$ also vanishes for sufficiently small perturbation $P$.) Choose a complement $D^{1} \subset C^{1}$ of im d $\mathrm{d}^{0}=\mathrm{kerd}^{1}$ and let $\Pi: C^{1} \rightarrow C^{1} / D^{1}$ be the projection. Then $\Pi \circ \mathrm{d}^{0}: C^{0} \rightarrow C^{1} / D^{1}$ is surjective and the restriction $\left.\mathrm{d}^{1}\right|_{D^{1}}: D^{1} \rightarrow C^{2}$ is an injective operator with a closed image. If 
$P^{i}: C^{i} \rightarrow C^{i+1}$ are sufficiently small then $\Pi \circ\left(\mathrm{d}^{0}+P^{0}\right): C^{0} \rightarrow C^{1} / D^{1}$ is still surjective and $\left.\left(\mathrm{d}^{1}+P^{1}\right)\right|_{D^{1}}: D^{1} \rightarrow C^{2}$ is still injective. From the latter and the identity $\left(\mathrm{d}^{1}+P^{1}\right) \circ\left(\mathrm{d}^{0}+P^{0}\right)=0$ it follows that $H_{P}^{0}=\operatorname{ker}\left(\mathrm{d}^{0}+P^{0}\right)$ agrees with the kernel of the surjective map $\Pi \circ\left(\mathrm{d}^{0}+P^{0}\right)$. Now let $D^{0} \subset C^{0}$ be a complement of $H^{0}=$ $\operatorname{ker} \mathrm{d}^{0}$, then $\left.\Pi \circ \mathrm{d}^{0}\right|_{D^{0}}: D^{0} \rightarrow C^{1} / D^{1}$ is bijective, and so is $\left.\Pi \circ\left(\mathrm{d}^{0}+P^{0}\right)\right|_{D^{0}}: D^{0} \rightarrow$ $C^{1} / D^{1}$ for sufficiently small $P^{0}$. Its inverse is an injective map $I_{P}: C^{1} / D^{1} \rightarrow C^{0}$ with image $D^{0}$ that depends continuously on $P$ and satisfies $\Pi \circ\left(\mathrm{d}^{0}+P^{0}\right) \circ I_{P}=\mathrm{Id}$. Now $\pi_{P}:=I_{P} \circ \Pi \circ\left(\mathrm{d}^{0}+P^{0}\right): C^{0} \rightarrow C^{0}$ is a projection, $\pi_{P} \circ \pi_{P}=\pi_{P}$, with $\operatorname{ker} \pi_{P}=\operatorname{ker}\left(\Pi \circ\left(\mathrm{d}^{0}+P^{0}\right)\right)=\operatorname{im}\left(1-\pi_{P}\right)$ and $\operatorname{im} \pi_{P}=\operatorname{im} I_{P}=D^{0}=\operatorname{ker}\left(1-\pi_{P}\right)$.

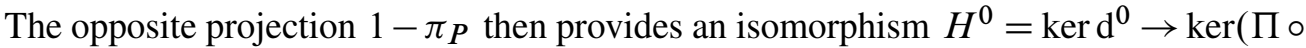
$\left.\left(\mathrm{d}^{0}+P^{0}\right)\right)=H_{P}^{0}$ that depends continuously on $P$.

\section{The linearized operator on $\mathbb{R} \times Y$}

Next, we shall use the above results on the Hessian to establish some basic properties of the linearized operator for (14). Let $I \subset \mathbb{R}$ be an open interval and $\mathbb{A}=A+\Phi \mathrm{d} s \in$ $\mathcal{A}(I \times Y)$ such that $\left.A(s)\right|_{\partial Y} \in \mathcal{L}$ for every $s \in I$. A $\mathfrak{g}$-valued 1 -form on $I \times Y$ has the form $\alpha+\varphi \mathrm{d} s$ with $\alpha(s) \in \Omega^{1}(Y, \mathfrak{g})$ and $\varphi(s) \in \Omega^{0}(Y, \mathfrak{g})$. Thus we shall identify $\Omega^{1}(I \times Y, \mathfrak{g})$ with the space of pairs $(\alpha, \varphi)$ of smooth maps $\alpha: I \rightarrow \Omega^{1}(Y, \mathfrak{g})$ and $\varphi: I \rightarrow \Omega^{0}(Y, \mathfrak{g})$. For any integer $k \geq 1$ and any $p>1$ let $W_{\mathbb{A}}^{k, p}\left(I \times Y, \mathrm{~T}^{*} Y \otimes \mathfrak{g}\right)$ denote the space of $W^{k, p}$-regular 1-forms $\alpha: I \times Y \rightarrow \mathrm{T}^{*} Y \otimes \mathfrak{g}$ that satisfy the boundary conditions

$$
\left.* \alpha(s)\right|_{\partial Y}=0,\left.\quad \alpha(s)\right|_{\partial Y} \in \mathrm{T}_{A(s)} \mathcal{L}
$$

for all $s \in I$. (The first equation arises from a gauge fixing condition.)

Remark 3.9 The boundary conditions (30) are meaningful for every $\alpha$ of class $W^{1, p}$ with $p>1$. In this case we have $\left.\alpha(s)\right|_{\partial Y} \in L^{p}\left(\Sigma, \mathrm{T}^{*} \Sigma \otimes \mathfrak{g}\right)$ for almost all $s \in I$, so there is a Hodge decomposition

$$
\left.\alpha(s)\right|_{\partial Y}=\alpha_{0}+\mathrm{d}_{\left.A(s)\right|_{\Sigma}} \xi+* \mathrm{~d}_{\left.A(s)\right|_{\Sigma}} \eta,
$$

and the second condition in (30) means that $\eta=0$ and $\alpha_{0} \in \mathrm{T}_{A(s) \mid{ }_{\Sigma}} \mathcal{L}$. In other words, $\left.\alpha(s)\right|_{\partial Y}$ lies in the $L^{p}$-closure of $\mathrm{T}_{\left.A(s)\right|_{\Sigma}} \mathcal{L}$. This $L^{p}$-closure is Lagrangian in the following sense: If $\alpha \in L^{p}\left(\Sigma, \mathrm{T}^{*} \Sigma \otimes \mathfrak{g}\right)$, then $\alpha$ lies in the $L^{p}$-closure of $\mathrm{T}_{A} \mathcal{L}$ if and only if $\int_{\Sigma}\langle\alpha \wedge \beta\rangle=0$ for all smooth $\beta \in \mathrm{T}_{A} \mathcal{L}$. (This extends the Lagrangian condition (6) to nonsmooth tangent vectors.)

On a general $4-$ manifold $X$, the linearized operator $\mathcal{D}_{\mathbb{A}}$ for (16) with a gauge fixing condition has the form

$$
\Omega^{1}(X, \mathfrak{g}) \rightarrow \Omega^{2,+}(X, \mathfrak{g}) \times \Omega^{0}(X, \mathfrak{g}): \widetilde{\alpha} \mapsto\left(\left(\mathrm{d}_{\mathbb{A}} \tilde{\alpha}+\mathrm{d} X_{f}(\mathbb{A}) \widetilde{\alpha}\right)^{+},-\mathrm{d}_{\mathbb{A}}^{*} \widetilde{\alpha}\right) .
$$


In the case $X=I \times Y$ we identify $\Omega^{2,+}(X, \mathfrak{g}) \times \Omega^{0}(X, \mathfrak{g})$ with the space of pairs of maps $I \rightarrow \Omega^{1}(Y, \mathfrak{g})$ and $I \rightarrow \Omega^{0}(Y, \mathfrak{g})$, using the formula

$$
\tilde{\alpha}=\frac{1}{2}(* \alpha(s)-\alpha(s) \wedge \mathrm{d} s)
$$

for self-dual 2-forms on $I \times Y$. With this notation the linearized operator

$$
\begin{aligned}
\mathcal{D}_{\mathbb{A}}: W_{\mathbb{A}}^{k, p}\left(I \times Y, \mathrm{~T}^{*} Y \otimes \mathfrak{g}\right) \times W^{k, p} & (I \times Y, \mathfrak{g}) \\
& \rightarrow W^{k-1, p}\left(I \times Y, \mathrm{~T}^{*} Y \otimes \mathfrak{g}\right) \times W^{k-1, p}(I \times Y, \mathfrak{g})
\end{aligned}
$$

for $I \times Y$ is given by

$$
\mathcal{D}_{\mathbb{A}}:=\nabla_{s}+\mathcal{H}_{A(s)},
$$

where $\nabla_{s}:=\partial_{s}+[\Phi, \cdot]$; explicitly,

$$
\mathcal{D}_{\mathbb{A}}\left(\begin{array}{c}
\alpha \\
\varphi
\end{array}\right)=\left(\begin{array}{c}
\nabla_{s} \alpha+* \mathrm{~d}_{A} \alpha+* \mathrm{~d} X_{f}(A) \alpha-\mathrm{d}_{A} \varphi \\
\nabla_{S} \varphi-\mathrm{d}_{A}^{*} \alpha
\end{array}\right) .
$$

Here we have dropped the argument $s$ in the notation, eg $\mathrm{d}_{A} \varphi$ stands for the path $s \mapsto \mathrm{d}_{A(s)} \varphi(s)$ of $\mathfrak{g}$-valued 1-forms on $Y$.

Remark 3.10 The formal adjoint operator has the form

$$
\mathcal{D}_{\mathbb{A}}^{*}=-\nabla_{s}+\mathcal{H}_{A(s)} .
$$

It is isomorphic to an operator of type $\nabla_{S}+\mathcal{H}_{A}$ via time reversal. Namely, if $\sigma:(-I) \times Y \rightarrow I \times Y$ denotes the reflection in the $s$-coordinate, then

$$
\mathcal{D}_{\mathbb{A}}^{*}(\beta, \psi) \circ \sigma=\mathcal{D}_{\sigma^{*} \mathbb{A}}(\beta \circ \sigma, \psi \circ \sigma)
$$

for every pair of smooth maps $\beta: I \rightarrow \Omega^{1}(Y, \mathfrak{g})$ and $\psi: I \rightarrow \Omega^{0}(Y, \mathfrak{g})$.

The following theorem provides the basic regularity (i) and estimate (ii) for the Fredholm theory of $\mathcal{D}_{\mathbb{A}}$ and will also be needed to prove exponential decay. The $L^{p}$-regularity has been established in [35] by techniques that do not extend to $p=2$. Here we prove the $L^{2}$-regularity using the analytic properties of the Hessian. A fundamental problem is that its domain varies with the connection, unlike in the closed case. The variation will be controlled in step 1 of the proof, using a trivialization of the tangent bundle of $\mathcal{L}$ in Appendix E. This control then allows to apply the general theory of Appendix A

Theorem 3.11 For every integer $k \geq 0$, every $p>1$, and every compact subinterval $J \subset I$ there is a constant $C$ such that the following holds.

(i) Assume $k=0$ and define $p^{*}:=p /(p-1)$. Let

$$
(\alpha, \varphi) \in L^{p}\left(I \times Y, \mathrm{~T}^{*} Y \otimes \mathfrak{g}\right) \times L^{p}(I \times Y, \mathfrak{g})
$$


and suppose that there is a constant $c$ such that

$$
\left|\int_{I \times Y}\left\langle\mathcal{D}_{\mathbb{A}}^{*}(\beta, \psi),(\alpha, \varphi)\right\rangle\right| \leq c\|(\beta, \psi)\|_{L^{p^{*}}(I \times Y)}
$$

for every compactly supported smooth map $(\beta, \psi): I \rightarrow \Omega^{1}(Y, \mathfrak{g}) \times \Omega^{0}(Y, \mathfrak{g})$ satisfying (30). Then $\left.(\alpha, \varphi)\right|_{J \times Y}$ is of class $W^{1, p}$ and satisfies the boundary condition (30) and the estimate

$$
\|(\alpha, \varphi)\|_{W^{1, p}(J \times Y)} \leq C\left(\left\|\mathcal{D}_{\mathbb{A}}(\alpha, \varphi)\right\|_{L^{p}(I \times Y)}+\|(\alpha, \varphi)\|_{L^{p}(I \times Y)}\right) .
$$

(ii) Assume $k \geq 1$. If $(\alpha, \varphi) \in W^{1, p}\left(I \times Y, \mathrm{~T}^{*} Y \otimes \mathfrak{g}\right) \times W^{1, p}(I \times Y$, g) satisfies (30) and $\mathcal{D}_{\mathbb{A}}(\alpha, \varphi)$ is of class $W^{k, p}$, then $\left.(\alpha, \varphi)\right|_{J \times Y}$ is of class $W^{k+1, p}$ and

$$
\|(\alpha, \varphi)\|_{W^{k+1, p}(J \times Y)} \leq C\left(\left\|\mathcal{D}_{\mathbb{A}}(\alpha, \varphi)\right\|_{W^{k, p}(I \times Y)}+\|(\alpha, \varphi)\|_{L^{p}(I \times Y)}\right) .
$$

Proof Using the estimates on the perturbation $\mathrm{d} X_{f}(A)$ in Proposition D.1 (iv) we may assume without loss of generality that $f=0$. Fix $s_{0} \in J$. We prove the result for a neighbourhood of $s_{0}$ in four steps.

Step 1 After shrinking I, there exists a family of bijective linear operators

$$
Q(s): \Omega^{1}(Y, \mathfrak{g}) \times \Omega^{0}(Y, \mathfrak{g}) \rightarrow \Omega^{1}(Y, \mathfrak{g}) \times \Omega^{0}(Y, \mathfrak{g}),
$$

parametrized by $s \in I$, such that the following holds.

(a) For every $s \in I$ and every $(\alpha, \varphi) \in \Omega^{1}(Y, \mathfrak{g}) \times \Omega^{0}(Y, \mathfrak{g})$

$$
(\alpha, \varphi) \in \operatorname{dom} \mathcal{H}_{A\left(s_{0}\right)} \quad \Longleftrightarrow \quad Q(s)(\alpha, \varphi) \in \operatorname{dom} \mathcal{H}_{A(s)} .
$$

(b) For every integer $k \geq 0$ and every $p>1$ the operator family $Q$ induces a continuous linear operator from $W_{\mathrm{loc}}^{k, p}\left(I \times Y, \mathrm{~T}^{*} Y \otimes \mathfrak{g}\right) \times W_{\mathrm{loc}}^{k, p}(I \times Y, \mathfrak{g})$ to itself.

Let $\mathcal{U} \subset \mathcal{A}(Y, \mathcal{L})$ be a neighbourhood of $A\left(s_{0}\right)$ that is open in the $\mathcal{C}^{0}$-topology and $\left\{Q_{A}\right\}_{A \in \mathcal{U}}$ be an operator family which satisfies the requirements of Theorem E.2. Shrink $I$ so that $A(s) \in \mathcal{U}$ for every $s \in I$. Then the operators $Q(s):=Q_{A(s)} \times \operatorname{Id}$ satisfy the requirements of Step 1.

Step 2 We prove (i) for $p=2$.

Abbreviate

$$
H:=L^{2}\left(Y, \mathrm{~T}^{*} Y \otimes \mathfrak{g}\right) \times L^{2}(Y, \mathfrak{g})
$$


and let $W(s) \subset H$ be the subspace of $(\alpha, \varphi) \in W^{1,2}\left(Y, \mathrm{~T}^{*} Y \otimes \mathfrak{g}\right) \times W^{1,2}(Y, \mathfrak{g})$ that satisfy the boundary conditions

$$
\left.* \alpha\right|_{\partial Y}=0,\left.\quad \alpha\right|_{\partial Y} \in \mathrm{T}_{A(s)} \mathcal{L}
$$

Let $Q$ be as in Step 1, so each $Q(s)$ induces an operator on $H$ that descends to a Hilbert space isomorphism from $W\left(s_{0}\right)$ to $W(s)$. Then, by Proposition 3.1 with $p=2$, the operator family $\mathcal{H}_{A(s)}: W(s) \rightarrow H$ satisfies the conditions (W1)-(W2) and (A1)-(A2) in Appendix A for every compact subinterval of $I$. Hence the estimate in (i) with $p=2$ follows from Lemma A.2 and a cutoff function argument, and the regularity statement follows from Theorem A.3.

Step 3 We prove (i) for $p \neq 2$.

The result follows from [35, Theorem C]. The intervals $I$ and $J$ can be replaced by $S^{1}$ by using cutoff functions, and one can interchange $\mathcal{D}_{\mathbb{A}}^{*}$ and $\mathcal{D}_{\sigma^{*} \mathbb{A}}$ in (32) by reversing time as in Remark 3.10. Then [35, Theorem C (iii)] implies that $(\alpha, \varphi) \circ \sigma$ is of class $W^{1, p}$ (with corresponding estimate). The same holds for $(\alpha, \varphi)$, and partial integration as in (18) implies that

$$
\begin{aligned}
C\|(\beta, \psi)\|_{L^{p^{*}}} & \geq\left|\int_{I \times Y}\left\langle\mathcal{D}_{\mathbb{A}}^{*}(\beta, \psi),(\alpha, \varphi)\right\rangle-\left\langle(\beta, \psi), \mathcal{D}_{\mathbb{A}}(\alpha, \varphi)\right\rangle\right| \\
& =\left|\int_{I \times \partial Y}\langle\alpha \wedge \beta\rangle+\langle * \alpha, \psi\rangle\right|
\end{aligned}
$$

Here we can choose any compactly supported $\left.\beta\right|_{I \times \partial Y}: I \rightarrow \mathrm{T}_{A} \mathcal{L} \subset \Omega^{1}(\partial Y, \mathfrak{g})$ and $\left.\psi\right|_{I \times \partial Y}: I \rightarrow \Omega^{0}(\partial Y, \mathfrak{g})$ and extend them to $I \times Y$ with $\|(\beta, \psi)\|_{L^{p^{*}}}$ arbitrarily small. Thus the above estimate implies that $\alpha$ satisfies the boundary conditions $\left.\alpha(s)\right|_{\partial Y} \in$ $\mathrm{T}_{A(s)} \mathcal{L}$ and $\left.* \alpha(s)\right|_{\partial Y}=0$.

\section{Step 4 We prove (ii).}

The assertion of (ii) continues to be meaningful for $k=0$; we prove it by induction on $k$. For $k=0$ the regularity statement holds by assumption and the estimate follows from (i). Fix an integer $k \geq 1$ and assume, by induction, that (ii) has been established with $k$ replaced by $k-1$. Let

$$
(\alpha, \varphi) \in W^{1, p}\left(I \times Y, \mathrm{~T}^{*} Y \otimes \mathfrak{g}\right) \times W^{1, p}(I \times Y, \mathfrak{g})
$$


such that (30) holds and

$$
(\beta, \psi):=\mathcal{D}_{\mathbb{A}}(\alpha, \varphi) \in W^{k, p}\left(I \times Y, \mathrm{~T}^{*} Y \otimes \mathfrak{g}\right) \times W^{k, p}(I \times Y, \mathfrak{g}) .
$$

Denote $\quad\left(\alpha^{\prime}, \varphi^{\prime}\right):=Q \partial_{s}\left(Q^{-1}(\alpha, \varphi)\right)$,

$$
\text { and } \quad\left(\beta^{\prime}, \psi^{\prime}\right):=Q\left(\partial_{s}\left(Q^{-1}(\beta, \psi)\right)-\left(\partial_{s}\left(Q^{-1} \mathcal{D}_{\mathbb{A}} Q\right)\right) Q^{-1}(\alpha, \varphi)\right) \text {. }
$$

Then $\left(\alpha^{\prime}, \varphi^{\prime}\right)$ satisfies the hypotheses of (i) and hence is of class $W^{1, p}$ and satisfies the boundary conditions (30). Thus

$$
\mathcal{D}_{\mathbb{A}}\left(\alpha^{\prime}, \varphi^{\prime}\right)=\left(\beta^{\prime}, \psi^{\prime}\right)
$$

is of class $W^{k-1, p}$. Hence, by the induction hypothesis, $\left(\alpha^{\prime}, \varphi^{\prime}\right)$ is of class $W^{k, p}$ and

$$
\begin{aligned}
\left\|\left(\alpha^{\prime}, \varphi^{\prime}\right)\right\|_{W^{k, p}(J \times Y)} & \leq C_{1}\left(\left\|\left(\beta^{\prime}, \psi^{\prime}\right)\right\|_{W^{k-1, p}(I \times Y)}+\left\|\left(\alpha^{\prime}, \varphi^{\prime}\right)\right\|_{L^{p}(I \times Y)}\right) \\
& \leq C_{2}\left(\|(\beta, \psi)\|_{W^{k, p}(I \times Y)}+\|(\alpha, \varphi)\|_{W^{k, p}(I \times Y)}\right) .
\end{aligned}
$$

Since $\left(\alpha^{\prime}, \varphi^{\prime}\right)=\left(\partial_{s} \alpha, \partial_{s} \varphi\right)-\left(\partial_{s} Q\right) Q^{-1}(\alpha, \varphi)$, this implies that $\left(\partial_{s} \alpha, \partial_{s} \varphi\right)$ is of class $W^{k, p}$ and

$$
\begin{aligned}
\left\|\left(\partial_{s} \alpha, \partial_{S} \varphi\right)\right\|_{W^{k, p}(J \times Y)} & \leq C_{3}\left(\left\|\mathcal{D}_{\mathbb{A}}(\alpha, \varphi)\right\|_{W^{k, p}(I \times Y)}+\|(\alpha, \varphi)\|_{W^{k, p}(I \times Y)}\right) \\
& \leq C_{4}\left(\left\|\mathcal{D}_{\mathbb{A}}(\alpha, \varphi)\right\|_{W^{k, p}(I \times Y)}+\|(\alpha, \varphi)\|_{L^{p}(I \times Y)}\right) .
\end{aligned}
$$

It remains to establish regularity and estimates for $(\alpha, \varphi)$ in $L^{p}\left(J, W^{k+1, p}(Y)\right)$. To see it note that $\mathcal{H}_{A}(\alpha, \varphi)=\mathcal{D}_{\mathbb{A}}(\alpha, \varphi)-\nabla_{s}(\alpha, \varphi)$ is of class $L^{p}\left(J, W^{k, p}(Y)\right)$. By Proposition 3.1, $(\alpha(s), \varphi(s)) \in W^{k+1, p}(Y)$ for almost every $s \in J$ and

$$
\begin{aligned}
\|(\alpha, \varphi)\|_{L^{p}\left(J, W^{k+1, p}(Y)\right)}^{p} & \\
& =\int_{J}\|(\alpha(s), \varphi(s))\|_{W^{k+1, p}(Y)}^{p} \mathrm{~d} s \\
& \leq C_{4} \int_{J}\left(\left\|\mathcal{H}_{A(s)}(\alpha(s), \varphi(s))\right\|_{W^{k, p}(Y)}^{p}+\|(\alpha(s), \varphi(s))\|_{L^{p}(Y)}^{p}\right) \mathrm{d} s \\
& \leq C_{5}\left(\left\|\mathcal{D}_{\mathbb{A}}(\alpha, \varphi)\right\|_{W^{k, p}(I \times Y)}^{p}+\|(\alpha, \varphi)\|_{L^{p}(I \times Y)}^{p}\right) .
\end{aligned}
$$

This completes the proof.

Remark 3.12 The proof of Theorem 3.11 carries over word for word to the case where the metric and perturbation on $Y$ depend smoothly on $s \in I$.

We finish this section with a complete description of the linearized operator for the trivial gradient flow line at an irreducible, nondegenerate critical point. 
Theorem 3.13 Let $A \in \mathcal{A}(Y, \mathcal{L})$ be a critical point of the perturbed Chern-Simons functional $\mathcal{C S}_{\mathcal{L}}+h_{f}$ such that $H_{A}^{0}=0$ and $H_{A, f}^{1}=0$. Then the operator

$$
\mathcal{D}_{A}:=\frac{\partial}{\partial s}+\mathcal{H}_{A}
$$

on $L^{p}\left(\mathbb{R} \times Y, \mathrm{~T}^{*} Y \otimes \mathfrak{g}\right) \times L^{p}(\mathbb{R} \times Y, \mathfrak{g})$ with domain

$$
\begin{aligned}
\operatorname{dom} \mathcal{D}_{A}:=\left\{(\alpha, \varphi) \in W^{1, p}\left(\mathbb{R} \times Y, \mathrm{~T}^{*} Y \otimes \mathfrak{g}\right)\right. & \times W^{1, p}(\mathbb{R} \times Y, \mathfrak{g}) \mid \\
& \left.\left.* \alpha(s)\right|_{\partial Y}=0,\left.\alpha(s)\right|_{\partial Y} \in \mathrm{T}_{A} \mathcal{L} \forall s \in \mathbb{R}\right\}
\end{aligned}
$$

is a Banach space isomorphism for every $p>1$.

Proof For $p=2$ it follows from [26, Theorem A] and Proposition 3.1 that $\mathcal{D}_{A}$ is a Fredholm operator of index zero; that it is bijective follows from the inequality (8) in [26]. Another argument is given in [10, Proposition 3.4]; it is based on the fact that $\mathcal{H}_{A}$ is a bijective self-adjoint Fredholm operator, and on the local $L^{2}$-regularity (Theorem 3.11). The case $p \neq 2$ can be reduced to the case $p=2$ by Donaldson's argument in [10, Proposition 3.21]; it uses in addition the local $L^{p}$-regularity in Theorem 3.11. (For an adaptation of Donaldson's argument to the symplectic case see [29, Lemma 2.4].)

\section{Operators on the product $S^{1} \times Y$}

In this section we study the anti-self-duality operator on $\mathrm{SU}(2)$-bundles over the product $S^{1} \times Y$ with Lagrangian boundary conditions. Our goal is, first, to establish a formula for the Fredholm index and, second, to prove that the relevant determinant line bundle is orientable. Both results are proved with the same technique. The problem can be reduced to the case of a suitable closed 3-manifold $Y \cup_{\Sigma} Y^{\prime}$ by means of an abstract argument involving the Gelfand-Robbin quotient.

Throughout we fix a compact connected oriented 3-manifold $Y$ with nonempty boundary $\partial Y=\Sigma$ and a gauge invariant, monotone Lagrangian submanifold $\mathcal{L} \subset \mathcal{A}(\Sigma)$ satisfying (L1)-(L2). We identify $S^{1} \cong \mathbb{R} / \mathbb{Z}$. Every gauge transformation $v: Y \rightarrow$ $\mathrm{G}=\mathrm{SU}(2)$ determines a principal SU(2)-bundle $P_{v} \rightarrow S^{1} \times Y$ defined by

$$
P_{v}:=\frac{\mathbb{R} \times Y \times \mathrm{G}}{\mathbb{Z}}, \quad[s, y, u] \equiv[s+1, y, v(y) u] .
$$

A connection on $P_{v}$ with Lagrangian boundary conditions is a pair of smooth maps $A: \mathbb{R} \rightarrow \mathcal{A}(Y, \mathcal{L})$ and $\Phi: \mathbb{R} \rightarrow \Omega^{0}(Y, \mathfrak{g})$ satisfying

$$
A(s+1)=v^{*} A(s), \quad \Phi(s+1)=v^{-1} \Phi(s) v .
$$


The space of such connections will be denoted by $\mathcal{A}\left(P_{v}, \mathcal{L}\right)$ and we write $\mathbb{A}=\Phi \mathrm{d} s+A$ or $(A, \Phi)$ for the elements of $\mathcal{A}\left(P_{v}, \mathcal{L}\right)$. The space

$$
\mathcal{A}\left(S^{1} \times Y, \mathcal{L}\right):=\left\{(v, \mathbb{A}) \mid \mathbb{A} \in \mathcal{A}\left(P_{v}, \mathcal{L}\right)\right\}
$$

is a groupoid. We will see that it has several connected components, corresponding to $\pi_{1}\left(\mathcal{L} / \mathcal{G}_{z}(\Sigma)\right)$ respectively the degree of $v:(Y, \partial Y) \rightarrow(\mathrm{G}, 11)$. A morphism from $\left(v_{0}, \mathbb{A}_{0}\right)$ to $\left(v_{1}, \mathbb{A}_{1}\right)$ is a smooth gauge transformation $u$ : $\mathbb{R} \rightarrow \mathcal{G}(Y)$ on $\mathbb{R} \times Y$ satisfying

$$
\begin{aligned}
v_{1} & =u(s)^{-1} v_{0} u(s+1), \\
\mathbb{A}_{1} & =u^{*} \mathbb{A}_{0} .
\end{aligned}
$$

We abbreviate (34) by $\left(v_{1}, \mathbb{A}_{1}\right)=: u^{*}\left(v_{0}, \mathbb{A}_{0}\right)$. In the case $v_{0}=v_{1}=v$ a map $u$ that satisfies the first equation in (34) is a gauge transformation on $P_{v}$. Since the gauge group $\mathcal{G}(Y)$ is connected there is, for every pair $v_{0}, v_{1} \in \mathcal{G}(Y)$, a gauge transformation $u: \mathbb{R} \rightarrow \mathcal{G}(Y)$ that satisfies the first equation in (34).

Fix a perturbation $X_{f}$. Then every pair $(v, \mathbb{A})=(v, A, \Phi) \in \mathcal{A}\left(S^{1} \times Y, \mathcal{L}\right)$ determines Sobolev spaces

$$
\begin{aligned}
W_{v}^{k, p}\left(S^{1} \times Y, \mathfrak{g}\right) & :=\left\{\varphi \in W_{\mathrm{loc}}{ }^{k, p}(\mathbb{R} \times Y, \mathfrak{g}) \mid \varphi(s+1)=v^{-1} \varphi(s) v\right\}, \\
W_{v}^{k, p}\left(S^{1} \times Y, \mathrm{~T}^{*} Y \otimes \mathfrak{g}\right) & :=\left\{\alpha \in W_{\mathrm{loc}^{k, p}}(\mathbb{R} \times Y, \mathfrak{g}) \mid \alpha(s+1)=v^{-1} \alpha(s) v\right\}, \\
W_{v, \mathbb{A}}^{k, p}\left(S^{1} \times Y, \mathrm{~T}^{*} Y \otimes \mathfrak{g}\right) & :=\left\{\alpha \in W_{v}^{k, p}\left(S^{1} \times Y, \mathrm{~T}^{*} Y \otimes \mathfrak{g}\right) \mid(30)\right\}
\end{aligned}
$$

and an anti-self-duality operator

$$
\begin{aligned}
\mathcal{D}_{v, \mathbb{A}}: W_{v, \mathbb{A}}^{k, p}\left(S^{1} \times Y, \mathrm{~T}^{*} Y \otimes \mathfrak{g}\right) & \times W_{v}^{k, p}\left(S^{1} \times Y, \mathfrak{g}\right) \\
& \rightarrow W_{v}^{k-1, p}\left(S^{1} \times Y, \mathrm{~T}^{*} Y \otimes \mathfrak{g}\right) \times W_{v}^{k-1, p}\left(S^{1} \times Y, \mathfrak{g}\right)
\end{aligned}
$$

given by $\mathcal{D}_{v, \mathbb{A}}:=\nabla_{s}+\mathcal{H}_{A(s)}$ respectively by (31) as in Section 3 .

Definition 4.1 The degree of a pair $(v, \mathbb{A})=(v, A, \Phi) \in \mathcal{A}\left(S^{1} \times Y, \mathcal{L}\right)$ is the integer

$$
\operatorname{deg}(v, \mathbb{A}):=-\frac{1}{4 \pi^{2}} \int_{0}^{1} \int_{Y}\left\langle F_{A} \wedge \partial_{S} A\right\rangle \mathrm{d} s .
$$


Remark 4.2 (i) The degree is an integer because it is the difference of the ChernSimons functionals. Explicitly,

$$
\begin{aligned}
\operatorname{deg}(v, \mathbb{A})= & -\frac{1}{8 \pi^{2}} \int_{0}^{1} \int_{\Sigma}\left\langle A \wedge \partial_{S} A\right\rangle \mathrm{d} s \\
& -\frac{1}{8 \pi^{2}}\left[\int_{Y}\left(\langle A \wedge \mathrm{d} A\rangle+\frac{1}{3}\langle A \wedge[A \wedge A]\rangle\right)\right]_{s=0}^{s=1} \\
= & \frac{1}{4 \pi^{2}}\left(\mathcal{C S}\left(A(0),\left.A\right|_{\Sigma} \# B\right)-\mathcal{C S}(A(1), B)\right) \\
\equiv & \frac{1}{4 \pi^{2}}\left(\mathcal{C S}_{\mathcal{L}}([A(0)])-\mathcal{C} \mathcal{S}_{\mathcal{L}}([A(0)])\right)=0 \in \mathbb{R} / \mathbb{Z} .
\end{aligned}
$$

Here $B:[0,1] \rightarrow \mathcal{L}$ is a smooth path from $B(0)=\left.A(1)\right|_{\Sigma}$ to $B(1)=0$ and $\left.A\right|_{\Sigma} \# B$ is the catenation of $\left.A\right|_{\Sigma}:[0,1] \rightarrow \mathcal{L}$ with $B$.

(ii) If $\left.v\right|_{\Sigma} \equiv \mathbb{1 l}$ then $\left.A(s+1)\right|_{\Sigma}=\left.A(s)\right|_{\Sigma}$ and, by (5),

$$
\operatorname{deg}(v, \mathbb{A})=\operatorname{deg}(v)-\frac{1}{8 \pi^{2}} \int_{0}^{1} \int_{\Sigma}\left\langle A \wedge \partial_{s} A\right\rangle \mathrm{d} s .
$$

The last term is the symplectic action of the loop $\mathbb{R} / \mathbb{Z} \rightarrow \mathcal{L}:\left.s \mapsto A(s)\right|_{\Sigma}$, multiplied by the factor $1 / 4 \pi^{2}$.

(iii) If $v \equiv \mathbb{1 l}$ and $\left.A(s)\right|_{\Sigma}=\left.u(s)^{*} A(0)\right|_{\Sigma}$ with $u(s+1)=u(s) \in \mathcal{G}(\Sigma)$ then $\operatorname{deg}(v, \mathbb{A})$ is minus the degree of the map $u: S^{1} \times \Sigma \rightarrow \mathrm{G}$; see Corollary 2.5.

Theorem 4.3 Fix $p>1$ and an integer $k \geq 1$. Then the following holds.

(i) Two pairs $(v, \mathbb{A}),\left(v^{\prime}, \mathbb{A}^{\prime}\right) \in \mathcal{A}\left(S^{1} \times Y, \mathcal{L}\right)$ belong to the same component of $\mathcal{A}\left(S^{1} \times Y, \mathcal{L}\right)$ if and only if they have the same degree.

(ii) For every pair $(v, \mathbb{A}) \in \mathcal{A}\left(S^{1} \times Y, \mathcal{L}\right)$ the operator $\mathcal{D}_{v, \mathbb{A}}$ is Fredholm and

$$
\operatorname{index}\left(\mathcal{D}_{v, \mathbb{A}}\right)=8 \operatorname{deg}(v, \mathbb{A}) .
$$

(iii) The determinant line bundle $\operatorname{det} \rightarrow \mathcal{A}\left(S^{1} \times Y, \mathcal{L}\right)$ with fibers $\operatorname{det}\left(\mathcal{D}_{v, \mathbb{A}}\right)$ is orientable.

(iv) Let $u: \mathbb{R} \rightarrow \mathcal{G}(Y)$ be a morphism from $(v, \mathbb{A})$ to $\left(v^{\prime}, \mathbb{A}^{\prime}\right)=\left(u^{*} v, u^{*} \mathbb{A}\right)$. Then $(v, \mathbb{A})$ and $\left(u^{*} v, u^{*} \mathbb{A}\right)$ have the same degree and the induced isomorphism

$$
u^{*}: \operatorname{det}\left(\mathcal{D}_{v, \mathbb{A}}\right) \rightarrow \operatorname{det}\left(\mathcal{D}_{\left(u^{*} v, u^{*} \mathbb{A}\right)}\right)
$$

is orientation preserving (ie the map on orientations agrees with the one induced by a homotopy). 
The proof of (ii) will be based on an identification of the index with the spectral flow of the Hessian. Both the index and orientation results in (ii)-(iv) require a description of the space of self-adjoint boundary conditions for the Hessian on a pair of domains with matching boundary. We will use it to homotop from Lagrangian boundary conditions to the diagonal (representing the closed case). More precisely, we will use the abstract setting of Appendix B.

We think of the div-grad-curl operator on $Y$ as an unbounded operator

$$
D:=\left(\begin{array}{cc}
* \mathrm{~d} & -\mathrm{d} \\
-\mathrm{d}^{*} & 0
\end{array}\right): W_{0} \rightarrow H
$$

on the Hilbert space

$$
H:=L^{2}\left(Y, \mathrm{~T}^{*} Y \otimes \mathfrak{g}\right) \oplus L^{2}(Y, \mathfrak{g})
$$

with the dense domain

$$
\operatorname{dom} D:=W_{0}:=W_{0}^{1,2}\left(Y, \mathrm{~T}^{*} Y \otimes \mathfrak{g}\right) \oplus W_{0}^{1,2}(Y, \mathfrak{g}) .
$$

With this domain $D$ is symmetric and injective and has a closed image, see Lemma 4.4 below. Hence $D$ satisfies the assumptions of Appendix B and thus defines a symplectic Hilbert space, the Gelfand-Robbin quotient

$$
V:=\operatorname{dom} D^{*} / \operatorname{dom} D=W / W_{0}, \quad \omega(\xi, \eta):=\left\langle D^{*} \xi, \eta\right\rangle-\left\langle\xi, D^{*} \eta\right\rangle,
$$

where $W:=\operatorname{dom} D^{*}$ is the domain of the adjoint operator $D^{*}$. The crucial property of the Gelfand-Robbin quotient is the fact that self-adjoint extensions of $D$ are in one-to-one correspondence with Lagrangian subspaces of $V$.

If $A \in \mathcal{A}(Y)$ is a smooth connection on $Y$ then the restricted (unperturbed) Hessian $\left.\mathcal{H}_{A}\right|_{W_{0}}: W_{0} \rightarrow H$ is an unbounded operator on $H$ with domain $W_{0}$. It is a compact perturbation of the div-grad-curl operator $D$. The next lemma shows how these operators fit into the setting of Appendix B.

Lemma 4.4 (i) For every smooth connection $A \in \mathcal{A}(Y)$ on $Y$ the operator

$$
\left.\mathcal{H}_{A}\right|_{W_{0}}: W_{0} \rightarrow H
$$

is symmetric, injective, and has a closed image. Its domain $W_{0}$ is dense in $H$, the graph norm of $\mathcal{H}_{A}$ on $W_{0}$ is equivalent to the $W^{1,2}$-norm, and the inclusion $W_{0} \rightarrow H$ is compact.

(ii) For every $A \in \mathcal{A}(Y)$ the domain of the dual operator $\left(\left.\mathcal{H}_{A}\right|_{W_{0}}\right)^{*}$ is equal to $W$ and the symplectic form on the quotient $W / W_{0}$ is given by

$$
\omega(\xi, \eta)=\int_{\partial Y}\langle\alpha \wedge \beta\rangle-\int_{\partial Y}\langle\varphi, * \beta\rangle+\int_{\partial Y}\langle * \alpha, \psi\rangle .
$$


for smooth elements $\xi=(\alpha, \varphi)$ and $\eta=(\beta, \psi)$ in $W$.

(iii) The kernel of $\left(\left.\mathcal{H}_{A}\right|_{W_{0}}\right)^{*}$ determines a Lagrangian subspace

$$
\Lambda_{0}(A):=\frac{\operatorname{ker}\left(\left.\mathcal{H}_{A}\right|_{W_{0}}\right)^{*}+W_{0}}{W_{0}} \subset V .
$$

If two connections $A, A^{\prime} \in \mathcal{A}(Y)$ coincide in a neighbourhood of the boundary $\partial Y$ then $\Lambda_{0}\left(A^{\prime}\right)$ is a compact perturbation of $\Lambda_{0}(A)$.

Proof The operator $\left.\mathcal{H}_{A}\right|_{W_{0}}$ is symmetric by (18) and it has a closed image by Lemma 3.5 (ii). To prove that it is injective let $(\alpha, \varphi) \in \operatorname{ker} \mathcal{H}_{A} \cap W_{0}$. Extend $A$ to an $S^{1}-$ invariant connection $\Xi$ on $S^{1} \times Y$ and $(\alpha, \varphi)$ to an $S^{1}$-invariant 1 -form $\xi=\alpha+\varphi \mathrm{d} s$ on $S^{1} \times Y$. Then $\mathrm{d}_{\Xi}^{+} \xi=0, \mathrm{~d}_{\Xi}^{*} \xi=0$, and $\xi$ vanishes on the (nonempty) boundary. Near the boundary we choose coordinates $(s, t, z) \in S^{1} \times[0, \varepsilon) \times \Sigma$ so that $(t, z)$ are normal geodesic coordinates on $Y$. Interchanging $s$ and $t$ we can first bring $\Xi$ into temporal gauge with respect to $t$ and then use Lemma 8.7 (ii) to deduce that $\xi$ vanishes near the boundary. Since $Y$ is connected it follows from an open and closed argument that $\xi$ vanishes identically. The graph norm of $\mathcal{H}_{A}$ on $W_{0}$ is given by (35) below. The boundary term vanishes on $W_{0}$ and hence this norm is equivalent to the $W^{1,2}$ norm. The compactness of the inclusion $W_{0} \rightarrow H$ follows from Rellich's theorem. This proves (i).

The domain of the dual operator and the symplectic form are independent of $A$ because the difference $\left.\mathcal{H}_{A}\right|_{W_{0}}-D=\left.\left(\mathcal{H}_{A}-\mathcal{H}_{0}\right)\right|_{W_{0}}: W_{0} \rightarrow H$ extends to a bounded self-adjoint operator from $H$ to itself. The formula for the symplectic form follows from (18).

Assertion (iii) follows from Lemma B.11. This uses the fact that the difference operator $\Delta:=\left(\mathcal{H}_{A} \mid W_{0}\right)^{*}-\left(\left.\mathcal{H}_{A^{\prime}}\right|_{W_{0}}\right)^{*}: W \rightarrow H$ is compact since it coincides with $\Delta \circ \iota \circ \Psi$. Here $\Psi: W \rightarrow W_{0}$ is a bounded map, given by multiplication with a cutoff function $\psi \in \mathcal{C}_{0}^{\infty}(Y,[0,1]),\left.\psi\right|_{\operatorname{supp}\left(A-A^{\prime}\right)} \equiv 1$, the inclusion $\iota: W_{0} \rightarrow H$ is compact by (i), and $\Delta: H \rightarrow H$ is bounded. This proves the lemma.

Remark 4.5 (i) The symplectic Hilbert space $(V, \omega)$ can be viewed as a space of boundary data for the Hessian, containing the space

$$
\frac{\Omega^{1}(Y, \mathfrak{g}) \times \Omega^{0}(Y, \mathfrak{g})}{W_{0} \cap\left(\Omega^{1}(Y, \mathfrak{g}) \times \Omega^{0}(Y, \mathfrak{g})\right)} \cong \Omega^{1}(\Sigma, \mathfrak{g}) \times \Omega^{0}(\Sigma, \mathfrak{g}) \times \Omega^{0}(\Sigma, \mathfrak{g})
$$

of smooth boundary data as a dense subspace; see Lemma 4.6 below. The isomorphism is by $[(\alpha, \varphi)] \mapsto\left(\left.\alpha\right|_{\partial Y},\left.\varphi\right|_{\partial Y}, *_{\Sigma}\left(\left.* \alpha\right|_{\partial Y}\right)\right)$. In this notation, an explicit formula for the symplectic form is given in Lemma 4.4 (ii). 
(ii) The space $\Omega^{1}(Y, \mathfrak{g}) \times \Omega^{0}(Y, \mathfrak{g})$ of smooth pairs $(\alpha, \varphi)$ is contained in the domain of the dual operator, and the restriction of $\left(\left.\mathcal{H}_{A}\right|_{W_{0}}\right)^{*}$ to this subspace agrees with $\mathcal{H}_{A}$. The graph norm on $\Omega^{1}(Y, \mathfrak{g}) \times \Omega^{0}(Y, \mathfrak{g}) \subset \operatorname{dom}\left(\left.\mathcal{H}_{A}\right|_{W_{0}}\right)^{*}$ is

$$
\begin{aligned}
\|(\alpha, \varphi)\|_{\left(\mathcal{H}_{A} \mid W_{0}\right)^{*}}^{2}= & \|(\alpha, \varphi)\|_{L^{2}(Y)}^{2}+\left\|\mathrm{d}_{A} \alpha\right\|_{L^{2}(Y)}^{2}+\left\|\mathrm{d}_{A}^{*} \alpha\right\|_{L^{2}(Y)}^{2} \\
& +\left\|\mathrm{d}_{A} \varphi\right\|_{L^{2}(Y)}^{2}+2 \int_{Y}\left\langle\varphi,\left[F_{A}, \alpha\right]\right\rangle-2 \int_{\partial Y}\left\langle\varphi, \mathrm{d}_{A} \alpha\right\rangle .
\end{aligned}
$$

The dual domain $W=\operatorname{dom}\left(\left.\mathcal{H}_{A}\right|_{W_{0}}\right)^{*}$ is the completion of $\Omega^{1}(Y, \mathfrak{g}) \times \Omega^{0}(Y, \mathfrak{g})$ with respect to this norm. It is bounded by the $W^{1,2}$-norm and hence

$$
W_{Y}^{1,2}:=W^{1,2}\left(Y, \mathrm{~T}^{*} Y \otimes \mathfrak{g}\right) \oplus W^{1,2}(Y, \mathfrak{g}) \subset W .
$$

Moreover, it follows from interior elliptic regularity that every element of the dual domain $W$ is of class $W^{1,2}$ on every compact subset of the interior of $Y$. However, $W$ is not contained in $W_{Y}^{1,2}$; see Lemma 4.6 below.

The next Lemma gives a precise description for the spaces $W$ and $V$, including some parts of weak regularity. However, our theory does not depend on the explicit description of these spaces. In our applications we only use the fact that the GelfandRobbin quotient is independent of the connection, see Lemma 4.4. In the following we slightly abuse notation and identify the Gelfand-Robbin quotient $V=W / W_{0}$ with the orthogonal complement of $W_{0}$ in $W$ in the graph norm of $D^{*}$. Remark B.1 (ii) shows that it is given by

$$
V=\left\{\xi \in \operatorname{dom} D^{*} \mid D^{*} \xi \in \operatorname{dom} D^{*}, D^{*} D^{*} \xi+\xi=0\right\} .
$$

Lemma 4.6 (i) The space $V$ admits an orthogonal Lagrangian splitting

$$
V=\Lambda_{0} \oplus \Lambda_{1}, \quad \Lambda_{0}:=D^{*} \Lambda_{1}, \quad \Lambda_{1}:=V \cap \operatorname{im} D,
$$

where $\Lambda_{0}$ is the orthogonal projection of the kernel of $D^{*}$ onto $V$.

(ii) The space $W$ admits an orthogonal splitting $W=W_{0} \oplus \Lambda_{0} \oplus \Lambda_{1}$, where $W_{0}$ and $\Lambda_{1}$ are closed subspaces of $W_{Y}^{1,2}$ and $\Lambda_{0}$ is a closed subspace of $H=: L_{Y}^{2}$.

(iii) The spaces of smooth elements are dense in $\Lambda_{0}, \Lambda_{1}, V$, and $W$ (with respect to the graph norm of $D^{*}$ ). The restriction map

$$
\xi=\left.(\alpha, \varphi) \mapsto \xi\right|_{\Sigma}:=\left(\left.\alpha\right|_{\Sigma},\left.\varphi\right|_{\Sigma}, *_{\Sigma}\left(\left.* \alpha\right|_{\Sigma}\right)\right)
$$

on the smooth elements extends continuously to $\Lambda_{0}$ and $\Lambda_{1}$. This gives rise to injective operators

$$
\Lambda_{0} \rightarrow W_{\Sigma}^{-1 / 2,2}, \quad \Lambda_{1} \rightarrow W_{\Sigma}^{1 / 2,2}
$$


with closed images. Here we denote $W_{\Sigma}^{-1 / 2,2}:=\left(W_{\Sigma}^{1 / 2,2}\right)^{*}$ and

$$
W_{\Sigma}^{1 / 2,2}:=W^{1 / 2,2}\left(\Sigma, T^{*} \Sigma \otimes \mathfrak{g}\right) \oplus W^{1 / 2,2}(\Sigma, \mathfrak{g}) \oplus W^{1 / 2,2}(\Sigma, \mathfrak{g}) .
$$

Proof The splitting in (i) is the one in Remark B.1 (iii) with $\Lambda_{1}=\Lambda_{0}^{\perp}$. To prove (ii) we examine the operator $D^{*} D$ of Lemma B.4. On smooth elements this is the LaplaceBeltrami operator. Hence its domain is

$$
\operatorname{dom}\left(D^{*} D\right)=\left\{\xi \in W_{0} \mid \sup _{\eta \in W_{0}} \frac{\langle D \xi, D \eta\rangle_{L^{2}}}{\|\eta\|_{L^{2}}}<\infty\right\}=W_{0} \cap W_{Y}^{2,2}
$$

by elliptic regularity. This implies that $\operatorname{dom} D^{*} \cap \operatorname{im} D=D\left(W_{0} \cap W_{Y}^{2,2}\right)$ is a closed subspace of $W_{Y}^{1,2}$. One can also think of $D^{*}$ as a bounded linear operator from $L_{Y}^{2}$ to $W_{Y}^{-1,2}:=\left(W_{0}\right)^{*}$, see the proof of Lemma B.4. Then the operator

$$
W_{Y}^{1,2} \rightarrow W_{Y}^{-1,2} \times W_{\Sigma}^{1 / 2,2}: \xi \mapsto\left(D^{*} D^{*} \xi+\xi,\left.\xi\right|_{\Sigma}\right)
$$

is bijective, by elliptic regularity and the Sobolev trace theorem, and $V \cap W_{Y}^{1,2}$ is the preimage of $\{0\} \times W_{\Sigma}^{1 / 2,2}$ under this operator. Hence $V \cap W_{Y}^{1,2}$ is also a closed subspace of $W_{Y}^{1,2}$ and so is the space

$$
\Lambda_{1}=\left(V \cap W_{Y}^{1,2}\right) \cap\left(\operatorname{dom} D^{*} \cap \operatorname{im} D\right) .
$$

Next, the kernel of $D^{*}$ is a closed subspace of $L_{Y}^{2}$ and hence, so is the space

$$
\Lambda_{0}=\left\{\xi-\left(11+D^{*} D\right)^{-1} \xi \mid \xi \in \operatorname{ker} D^{*}\right\} .
$$

See Remark B.1 (ii) for the projection $W \rightarrow V$; the formula simplifies for $\xi \in \operatorname{ker} D^{*}$. This proves (ii).

We prove that the spaces of smooth elements are dense in $\Lambda_{0}, \Lambda_{1}, V$, and $W$. Any element in $\Lambda_{1}$ can be approximated by a smooth sequence in $\Lambda_{1}$ : The $W^{1,2}-$ approximation by any smooth sequence converges in the graph norm of $D^{*}$ and projects under the map $\Pi_{0}$ in Remark B.5 to a convergent smooth sequence in $\Lambda_{1}$. Since $\Lambda_{0}=D^{*} \Lambda_{1}$, this shows that the smooth elements are dense in $\Lambda_{0}$ as well as in $W=W_{0} \oplus \Lambda_{0} \oplus \Lambda_{1}$.

That the restriction map (36) extends to an injective bounded linear operator from $\Lambda_{1}$ onto a closed subspace of $W_{\Sigma}^{1 / 2,2}$ follows by restricting the isomorphism (37) to the closed subspace $\Lambda_{1}$ of $V \cap W_{Y}^{1,2}$. Next we prove that the map (36) sends $\Lambda_{0}$ to a closed subspace of $W^{-1 / 2,2}(\Sigma)$. For this it is convenient to use the following norms 
for $\xi \in W$ :

$$
\left\|\left.\xi\right|_{\Sigma}\right\|_{W_{\Sigma}^{-1 / 2,2}}:=\sup _{\eta \in W_{Y}^{1,2}} \frac{\omega(\xi, \eta)}{\|\eta\|_{W_{Y}^{1,2}}}, \quad\|\xi\|_{D^{*}}:=\sqrt{\|\xi\|_{L_{Y}^{2}}^{2}+\left\|D^{*} \xi\right\|_{L_{Y}^{2}}^{2}}
$$

By definition there is a constant $c>0$ such that

$$
\left\|\left.\xi\right|_{\Sigma}\right\|_{W_{\Sigma}^{-1 / 2,2}} \leq c\|\xi\|_{D^{*}}
$$

for every $\xi \in W$. Thus (36) is a bounded linear operator from $W$ to $W_{\Sigma}^{-1 / 2,2}$. Moreover, $\Lambda_{1}$ is complete both with respect to the graph norm of $D^{*}$ and the $W^{1,2}$ norm, and the former is bounded above by the latter. Hence, by the open mapping theorem, there is a constant $\delta>0$ such that

$$
\|\eta\|_{D^{*}} \geq \delta\|\eta\|_{W_{Y}^{1,2}} \quad \forall \eta \in \Lambda_{1}
$$

Now let $\xi \in \Lambda_{0}$ be given. Then $D^{*} \xi \in \Lambda_{1} \subset W_{Y}^{1,2}$ and hence

$$
\left\|\left.\xi\right|_{\Sigma}\right\|_{W_{\Sigma}^{-1 / 2,2} \geq \delta} \sup _{\eta \in W_{Y}^{1,2}} \frac{\omega(\xi, \eta)}{\|\eta\|_{D^{*}}} \geq \delta \frac{\omega\left(\xi, D^{*} \xi\right)}{\left\|D^{*} \xi\right\|_{D^{*}}}=\delta\|\xi\|_{D^{*}}
$$

Since $\Lambda_{0}$ is a closed subspace of $W$, the operator $\Lambda_{0} \rightarrow W_{\Sigma}^{-1 / 2,2}:\left.\xi \mapsto \xi\right|_{\Sigma}$ is injective and has a closed image. This proves the lemma.

Remark 4.7 The dual domain $W$ admits another orthogonal splitting

$$
W=\left(\operatorname{dom} D^{*} \cap \operatorname{im} D\right) \oplus \operatorname{ker} D^{*}
$$

where $\operatorname{dom} D^{*} \cap \operatorname{im} D$ is a closed subspace of $W_{Y}^{1,2}$ and the kernel of $D^{*}$ is a closed subspace of $L_{Y}^{2}$. It can be described as the image under $D^{*}$ of the space of harmonic pairs $\xi=(\alpha, \beta) \in W_{Y}^{1,2}$ :

$$
\text { ker } D^{*}=\left\{\left(* \mathrm{~d} \alpha-\mathrm{d} \varphi,-\mathrm{d}^{*} \alpha\right) \mid(\alpha, \varphi) \in W_{Y}^{1,2}, \mathrm{~d}^{*} \mathrm{~d} \alpha+\mathrm{dd}^{*} \alpha=0, \mathrm{~d}^{*} \mathrm{~d} \varphi=0\right\} .
$$

This can also be used to prove that the restriction map (36) maps the kernel of $D^{*}$ to $W_{\Sigma}^{-1 / 2,2}$ : If $\varphi$ is a $W^{1,2}$ harmonic function on $Y$ then its restriction to the boundary is of class $W^{1 / 2,2}$ and its normal derivative on the boundary is of class $W^{-1 / 2,2}$.

Yet another splitting of $W$ can be obtained from eigenspace decompositions along the lines of Atiyah-Patodi-Singer [5]. The operator $D$ has the form $J\left(\partial_{t}+B\right)$ near the boundary, where $J^{2}=-11$ and $B$ is a self-adjoint first order Fredholm operator over $\Sigma$. The decomposition involves the eigenspaces of $B$ [8]. 
Proof of Theorem 4.3 It suffices to prove the theorem for $X_{f}=0$ because any two perturbations are homotopic and result in compact perturbations of the operators $\mathcal{D}_{v, \mathbb{A}}$ and hence in isomorphic determinant line bundles.

We prove (i). By Lemma 2.4 the degree depends only on the homotopy class of $(v, \mathbb{A})$. Given such a pair, there is a smooth path $[0,1] \rightarrow \mathcal{G}(Y): \tau \mapsto v^{\tau}$ with $v^{0}=v$ and $v^{1}=11$, because $\mathcal{G}(Y)$ is connected. Let $u^{\tau}: \mathbb{R} \rightarrow \mathcal{G}(Y)$ be the smooth path of gauge transformations constructed in Lemma 4.8 below with $X=$ pt and define

$$
\mathbb{A}^{\tau}:=\left(u^{\tau}\right)^{*} \mathbb{A} \text {. }
$$

Then $\tau \mapsto\left(v^{\tau}, \mathbb{A}^{\tau}\right)$ is a smooth path in $\mathcal{A}\left(S^{1} \times Y, \mathcal{L}\right)$ connecting $\left(v^{0}, \mathbb{A}^{0}\right)=(v, \mathbb{A})$ to a pair of the form $\left(\mathbb{1 1}, A^{1}\right)$. Hence we may assume without loss of generality that $v=v^{\prime}=\mathbb{1}$ and $\mathbb{A}, \mathbb{A}^{\prime} \in \mathcal{A}(P, \mathcal{L})$ where $P=P_{\mathbb{1}}=S^{1} \times Y \times \mathrm{G}$. Now the map

$$
\mathcal{A}(P, \mathcal{L}) \rightarrow C^{\infty}\left(S^{1}, \mathcal{L}\right):\left.\mathbb{A} \mapsto A\right|_{S^{1} \times \Sigma}
$$

is a homotopy equivalence. Hence (i) follows from the fact that, by (L2), every loop in $\mathcal{L}$ is homotopic to a loop of the form $\mathbb{R} / \mathbb{Z} \rightarrow \mathcal{L}: s \mapsto u(s)^{*} A_{0}$ with $u(s+1)=u(s) \in \mathcal{G}(\Sigma)$, and that the homotopy class of such a loop is characterized by the degree of the map $u: S^{1} \times \Sigma \rightarrow$ G.

We prove (ii). That the operator $\mathcal{D}_{v, \mathbb{A}}$ has a finite dimensional kernel and a closed image follows immediately from the estimate in Theorem 3.11 (ii) and Rellich's theorem (see [21, Lemma A.1.1]). That it has a finite dimensional cokernel follows from the regularity results in Theorem 3.11 and Remark 3.10. (The dual operator has a finite dimensional kernel.) Thus we have proved that $\mathcal{D}_{v, \mathbb{A}}$ is a Fredholm operator for every pair $(v, \mathbb{A}) \in \mathcal{A}\left(S^{1} \times Y, \mathcal{L}\right)$. The regularity theory in Theorem 3.11 also shows that its kernel and cokernel, and hence also the Fredholm index, are independent of $k$ and $p$. Moreover, the Fredholm index depends only on the homotopy class of $(v, \mathbb{A})$; to see this one can use the argument in the proof of Step 1 in Theorem 3.11 to reduce the problem to small deformations with constant domain and then use the stability properties of the Fredholm index. So by (i) it suffices to consider one pair $(v, \mathbb{A})$ in each degree. Hence we can assume

$$
\left.v\right|_{N}=1, \quad \Phi=0,\left.\quad A(s)\right|_{N}=0
$$

for all $s$ and an open neighbourhood $N \subset Y$ of $\partial Y$. Then $\operatorname{deg}(v, \mathbb{A})=\operatorname{deg}(v)$. Choose a handle body $Y^{\prime}$ with $\partial Y^{\prime}=\bar{\Sigma}$ and extend $A(s)$ smoothly by the trivial connection on $Y^{\prime}$ to obtain a smooth connection $\widetilde{A}(s)$ on the closed 3-manifold

$$
\tilde{Y}:=Y \cup_{\Sigma} Y^{\prime}
$$


for every $s$. Note that $\widetilde{A}(s+1)=\widetilde{v}^{*} \widetilde{A}(s)$, where $\tilde{v} \in \mathcal{G}(\tilde{Y})$ agrees with $v$ on $Y$ and is equal to 11 on $Y^{\prime}$. Let $\mathcal{H}_{0}^{\prime}$ denote the Hessian on $Y^{\prime}$ (at the trivial connection) and $\mathcal{H}_{A(s)}$ the Hessian on $Y$, both with the same boundary Lagrangian $T_{0} \mathcal{L}$. These are self-adjoint Fredholm operators, by Proposition 3.1. The Hessian (17) over the closed manifold $\tilde{Y}$ will be denoted by $\widetilde{\mathcal{H}} \widetilde{A}(s)$. Choose $\varepsilon>0$ such that the operators $\mathcal{H}_{0}^{\prime}+\varepsilon \mathrm{Id}$ $\mathcal{H}_{A(0)}+\varepsilon \mathrm{Id}$, and $\widetilde{\mathcal{H}} \widetilde{A}(0)+\varepsilon \mathrm{Id}$ are all bijective. We shall introduce the spectral flow $\mu_{\text {spec }}$ (as defined in Appendix A) and prove that

$$
\begin{aligned}
\operatorname{index}\left(\mathcal{D}_{v, \mathbb{A}}\right) & =\mu_{\text {spec }}\left(\left\{\left(\mathcal{H}_{A(s)}+\varepsilon \mathrm{Id}\right) \oplus\left(\mathcal{H}_{0}^{\prime}+\varepsilon \mathrm{Id}\right)\right\}_{s \in[0,1]}\right) \\
& =\mu_{\text {spec }}\left(\{\widetilde{\mathcal{H}} \widetilde{A}(s)+\varepsilon \operatorname{Id}\}_{s \in[0,1]}\right) \\
& =\operatorname{index}\left(\mathcal{D}_{\widetilde{v}, \widetilde{\mathbb{A}}}\right)=8 \operatorname{deg}(\widetilde{v})=8 \operatorname{deg}(v, \mathbb{A}) .
\end{aligned}
$$

Here $\mathcal{D}_{\widetilde{v}, \widetilde{\mathbb{A}}}=\nabla_{\widetilde{S}}+\widetilde{\mathcal{H}} \widetilde{A}(s)$ denotes the anti-self-duality operator on the twisted bundle $P_{\widetilde{v}}$ over $S^{1} \times \tilde{Y}$.

To prove (38) we may assume $k=1$ and $p=2$. In this case the first and third equations follow from Theorem A.5, the fourth equation follows from the Atiyah-Singer index theorem (the second Chern class of the principal bundle $P_{\widetilde{v}} \rightarrow S^{1} \times \widetilde{Y}$ is the degree of $\widetilde{v}$ ), and the last equation is obvious from the definitions. To prove the second equation in (38) consider the operator family

$$
D(s):=\left(\mathcal{H}_{A(s)}+\varepsilon \mathrm{Id}\right) \oplus\left(\mathcal{H}_{0}^{\prime}+\varepsilon \mathrm{Id}\right)
$$

on the Hilbert space

$$
H:=L^{2}\left(Y, \mathrm{~T}^{*} Y \otimes \mathfrak{g}\right) \oplus L^{2}(Y, \mathfrak{g}) \oplus L^{2}\left(Y^{\prime}, \mathrm{T}^{*} Y^{\prime} \otimes \mathfrak{g}\right) \oplus L^{2}\left(Y^{\prime}, \mathfrak{g}\right)
$$

with the constant dense domain dom $D(s)=W_{0}$, where

$$
W_{0}:=W_{0}^{1,2}\left(Y, \mathrm{~T}^{*} Y \otimes \mathfrak{g}\right) \oplus W_{0}^{1,2}(Y, \mathfrak{g}) \oplus W_{0}^{1,2}\left(Y^{\prime}, \mathrm{T}^{*} Y^{\prime} \otimes \mathfrak{g}\right) \oplus W_{0}^{1,2}\left(Y^{\prime}, \mathfrak{g}\right) .
$$

As in Remark 4.5, this choice of domain makes $D(s)$ closed, symmetric, and injective. Moreover, the Gelfand-Robbin quotient and its symplectic structure

$$
V:=\operatorname{dom} D(s)^{*} / \operatorname{dom} D(s)=W / W_{0}
$$

are independent of $s$. Now, by Appendix B, self-adjoint extensions of $D(s)$ are in one-to-one correspondence with Lagrangian subspaces of $V$. The operators in the first row of (38) all correspond to the Lagrangian subspace

$$
\Lambda_{1}:=\left\{\begin{array}{l|l}
\left(\alpha, \varphi, \alpha^{\prime}, \varphi^{\prime}\right) \in W^{1,2} & \begin{array}{c}
\left.* \alpha\right|_{\partial Y},\left.* \alpha^{\prime}\right|_{\partial Y^{\prime}}=0, \\
\left.\alpha\right|_{\partial Y},\left.\alpha^{\prime}\right|_{\partial Y^{\prime}} \in \mathrm{T}_{0} \mathcal{L}
\end{array}
\end{array}\right\} / W_{0} \subset V,
$$


where $W^{1,2}:=W^{1,2}\left(Y, \mathrm{~T}^{*} Y \otimes \mathfrak{g} \oplus \mathfrak{g}\right) \times W^{1,2}\left(Y^{\prime}, \mathrm{T}^{*} Y^{\prime} \otimes \mathfrak{g} \oplus \mathfrak{g}\right) \subset W$. The operators in the second row of (38) all correspond to the "diagonal"

$$
\Lambda_{2}:=\left\{\begin{array}{l|l}
\left(\alpha, \varphi, \alpha^{\prime}, \varphi^{\prime}\right) \in W^{1,2} & \begin{array}{c}
\left.\varphi\right|_{\partial Y}=\left.\varphi^{\prime}\right|_{\partial Y^{\prime}}, \\
\left.\alpha\right|_{\partial Y}=\left.\alpha^{\prime}\right|_{\partial Y^{\prime}}, \\
\left.* \alpha\right|_{\partial Y}=\left.* \alpha^{\prime}\right|_{\partial Y^{\prime}}
\end{array}
\end{array}\right\} / W_{0} \subset V .
$$

For $i=1,2$ and $s \in \mathbb{R}$ let $D(s)_{\Lambda_{i}}: \operatorname{dom} D(s)_{\Lambda_{i}} \rightarrow H$ denote the restriction of $D(s)^{*}$ to the preimage of $\Lambda_{i}$ under the projection $W \rightarrow W / W_{0}$. Then $D(s)_{\Lambda_{i}}$ is self-adjoint. Moreover, we have $D(s+1)=Q^{-1} D(s) Q$, where $Q: H \rightarrow H$ is given by conjugation with the gauge transformation $v$ and satisfies $\xi-Q \xi \in W_{0}$ for all $\xi \in W$ since $v \equiv \mathbb{1}$ near $\partial Y$. This implies that

$$
\Lambda_{0}:=\left(\operatorname{ker} D(0)^{*} \oplus W_{0}\right) / W_{0}=\left(\operatorname{ker} D(1)^{*} \oplus W_{0}\right) / W_{0} .
$$

Then, by the choice of $\varepsilon$, the Lagrangian subspaces $\Lambda_{1}$ and $\Lambda_{2}$ are transverse to $\Lambda_{0}$. Moreover, they are compact perturbations of $\Lambda_{0}^{\perp}$ by Lemma B.10, since the graph norm on dom $D(s)_{\Lambda_{i}}$ is equivalent to the $W^{1,2}$-norm; see (35). The second identity in (38) follows from Remark B.14, which asserts that the spectral flow of $\left\{D(s)_{\Lambda}\right\}_{s \in[0,1]}$ is independent of the Lagrangian subspace $\Lambda \subset V$ that is transverse to $\Lambda_{0}$ and a compact perturbation of $\Lambda_{0}^{\perp}$. This proves (38) and thus (ii).

We prove (iii) and (iv). That two isomorphic pairs $\left(v_{0}, \mathbb{A}_{0}\right)$ and $\left(v_{1}, \mathbb{A}_{1}\right)=u^{*}\left(v_{0}, \mathbb{A}_{0}\right)$ have the same degree follows from (ii) and the fact that conjugation by $u$ identifies kernel and cokernel of the operator $\mathcal{D}_{v_{0}, \mathbb{A}_{0}}$ with kernel and cokernel of $\mathcal{D}_{v_{1}, \mathbb{A}_{1}}$. For every $(v, \mathbb{A}) \in \mathcal{A}\left(S^{1} \times Y, \mathcal{L}\right)$ denote by $\operatorname{Or}\left(\mathcal{D}_{v, \mathbb{A}}\right)$ the two element set of orientations of $\operatorname{det}\left(\mathcal{D}_{v, \mathbb{A}}\right)$. Then the remaining assertions in (iii) and (iv) can be rephrased as follows.

Claim Let $\left\{\left(v_{\lambda}, \mathbb{A}_{\lambda}\right)\right\}_{0 \leq \lambda \leq 1}$ be a smooth path in $\mathcal{A}\left(S^{1} \times Y, \mathcal{L}\right)$ and $u$ : $\mathbb{R} \rightarrow \mathcal{G}(Y)$ be a morphism from $\left(v_{0}, \mathbb{A}_{0}\right)$ to $\left(v_{1}, \mathbb{A}_{1}\right)$. Then the isomorphism

$$
u^{*}: \operatorname{Or}\left(\mathcal{D}_{v_{0}, \mathbb{A}_{0}}\right) \rightarrow \operatorname{Or}\left(\mathcal{D}_{v_{1}, \mathbb{A}_{1}}\right)
$$

agrees with the isomorphism induced by the path $\lambda \mapsto\left(v_{\lambda}, \mathbb{A}_{\lambda}\right)$.

When $u \equiv \mathbb{1}$, the claim asserts that the automorphism of $\operatorname{det}\left(\mathcal{D}_{v_{0}, \mathbb{A}_{0}}\right)$ induced by a loop in $\mathcal{A}\left(S^{1} \times Y, \mathcal{L}\right)$ is orientation preserving and hence the determinant bundle over $\mathcal{A}\left(S^{1} \times Y, \mathcal{L}\right)$ is orientable. Throughout we write $\mathbb{A}_{\lambda}=\Phi_{\lambda}(s) \mathrm{d} s+A_{\lambda}(s)$ We prove the claim in five steps.

Step 1 It suffices to assume that $v_{\lambda}=11$ for every $\lambda$. 
Since $\mathcal{G}(Y)$ is connected, there exists a smooth homotopy $[0,1] \times[0,1] \rightarrow \mathcal{G}(Y)$ : $(\tau, \lambda) \mapsto v_{\lambda}^{\tau}$ from $v_{\lambda}^{0}=v_{\lambda}$ to $v_{\lambda}^{1}=11$. By Lemma 4.8 below with $X=[0,1]$, there exists a smooth map $[0,1] \times[0,1] \times \mathbb{R} \rightarrow \mathcal{G}(Y):(\tau, \lambda, s) \mapsto u_{\lambda}^{\tau}(s)$ such that

$$
v_{\lambda}^{\tau}=u_{\lambda}^{\tau}(s)^{-1} v_{\lambda} u_{\lambda}^{\tau}(s+1), \quad u_{\lambda}^{0}(s)=\mathbb{1} .
$$

Define

$$
\mathbb{A}_{\lambda}^{\tau}:=\left(u_{\lambda}^{\tau}\right)^{*} \mathbb{A}_{\lambda}, \quad u^{\tau}:=\left(u_{0}^{\tau}\right)^{-1} u u_{1}^{\tau} .
$$

Then $\left(v_{\lambda}^{\tau}\right)^{*} A_{\lambda}^{\tau}(s)=A_{\lambda}^{\tau}(s+1), \mathbb{A}_{1}^{\tau}=\left(u^{\tau}\right)^{*} \mathbb{A}_{0}^{\tau}$, and $v_{1}^{\tau}=u^{\tau}(s)^{-1} v_{0}^{\tau} u^{\tau}(s+1)$. Hence $\left(v_{\lambda}^{\tau}, \mathbb{A}_{\lambda}^{\tau}\right) \in \mathcal{A}\left(S^{1} \times Y, \mathcal{L}\right)$ for all $\tau$ and $\lambda$, and $u^{\tau}$ is a morphism from $\left(v_{0}^{\tau}, \mathbb{A}_{0}^{\tau}\right)$ to $\left(v_{1}^{\tau}, \mathbb{A}_{1}^{\tau}\right)$ for every $\tau$. By continuity, the claim holds for $\tau=0$ if and only if it holds for $\tau=1$. Since $v_{\lambda}^{1}=11$ for every $\lambda$, this proves Step 1 .

Step 2 It suffices to assume that $v_{\lambda}=\mathbb{1}$ and $\left.u\right|_{S^{1} \times \Sigma}=\mathbb{1}$.

By Step 1 we can assume $v_{\lambda}=11$. The restriction of the map $u: S^{1} \times Y \rightarrow \mathrm{G}$ to the boundary has degree zero (see eg [22, Section 5,Lemma 1]). Hence there exists a smooth path $[0,1] \rightarrow \mathcal{G}(P): \tau \mapsto u^{\tau}$ such that $u^{0}=u$ and $\left.u^{1}\right|_{S^{1} \times \Sigma}=\mathbb{1 1}$. Composing the paths $\left\{\mathbb{A}_{\lambda}\right\}_{0 \leq \lambda \leq 1}$ and $\left\{\left(u^{\lambda \tau}\right)^{*} \mathbb{A}_{0}\right\}_{0 \leq \lambda \leq 1}$ we obtain a homotopy of homotopies $\tau \mapsto\left\{\mathbb{A}_{\lambda}^{\tau}\right\}_{0 \leq \lambda \leq 1}$ with $\mathbb{A}_{\lambda}^{0}=\mathbb{A}_{\lambda}$ and $\mathbb{A}_{1}^{\tau}=\left(u^{\tau}\right)^{*} \mathbb{A}_{0}^{\tau}$. Hence Step 2 follows as in Step 1 by continuity.

Step 3 Using (L2) we see that it suffices to assume that $v_{\lambda}=\mathbb{1},\left.u\right|_{S^{1} \times \Sigma}=11$, and there exists a smooth map $[0,1] \times S^{1} \rightarrow \mathcal{G}_{z}(\Sigma):(\lambda, s) \mapsto w_{\lambda}(s)$ satisfying $\left.A_{\lambda}(s)\right|_{\Sigma}=$ $w_{\lambda}(s)^{-1} \mathrm{~d} w_{\lambda}(s)$ and $w_{\lambda}(s+1)=w_{\lambda}(s), w_{0}(s)=w_{1}(s), w_{0}(0)=11$.

By Step 2 we can assume $v_{\lambda}=\mathbb{1 1}$ and $\left.u\right|_{S^{1} \times \partial Y}=\mathbb{1}$. Then $A_{\lambda}(s+1)=A_{\lambda}(s)$ and $A_{0}(s)=A_{1}(s)$ for all $s$ and $\lambda$. Since $\mathcal{L} / \mathcal{G}_{z}(\Sigma)$ is connected and simply connected, the loops $[0,1] \rightarrow \mathcal{L}:\left.\lambda \mapsto A_{\lambda}(0)\right|_{\Sigma}$ and $S^{1} \rightarrow \mathcal{L}:\left.s \mapsto A_{0}(s)\right|_{\Sigma}$ are homotopic to loops in the based gauge equivalence class of the zero connection in $\mathcal{L}$. This implies that there is a smooth homotopy $[0,1]^{2} \times S^{1} \rightarrow \mathcal{L}:(\tau, \lambda, s) \mapsto B_{\lambda}^{\tau}(s)$ of homotopies of loops, satisfying

$$
B_{\lambda}^{\tau}(s+1)=B_{\lambda}^{\tau}(s), \quad B_{0}^{\tau}(s)=B_{1}^{\tau}(s),
$$

starting at $B_{\lambda}^{0}(s)=\left.A_{\lambda}(s)\right|_{\Sigma}$ and ending at a homotopy of loops satisfying

$$
B_{\lambda}^{1}(0), B_{0}^{1}(s) \in\left\{w^{-1} \mathrm{~d} w \mid w \in \mathcal{G}_{z}(\Sigma)\right\} .
$$

The composition of the map $[0,1]^{2} \rightarrow \mathcal{L}:(\lambda, s) \mapsto B_{\lambda}^{1}(s)$ with the projection $\mathcal{L} \rightarrow$ $\mathcal{L} / \mathcal{G}_{z}(\Sigma)$ maps the boundary to a point. Since $\pi_{2}\left(\mathcal{L} / \mathcal{G}_{z}(\Sigma)\right)=0$ the homotopy $\tau \mapsto$ $B^{\tau}$ can be extended to the interval $0 \leq \tau \leq 2$ so that $B_{\lambda}^{2}(s)=w_{\lambda}(s)^{-1} \mathrm{~d} w_{\lambda}(s)$. This 
determines the map $[0,1] \times \mathbb{R} \rightarrow \mathcal{G}_{z}(\Sigma):(\lambda, s) \mapsto w_{\lambda}(s)$ uniquely, hence $w$ satisfies the requirements of Step 3. Since the restriction map $\mathcal{A}(Y, \mathcal{L}) \rightarrow \mathcal{L}$ is a homotopy equivalence, there exists a smooth homotopy $[0,2] \times[0,1] \rightarrow \mathcal{A}\left(P_{\mathbb{1}}, \mathcal{L}\right):(\tau, \lambda) \mapsto \mathbb{A}_{\lambda}^{\tau}$ with $\mathbb{A}_{1}^{\tau}=u^{*} \mathbb{A}_{0}^{\tau}$ from $\mathbb{A}_{\lambda}^{0}=\mathbb{A}_{\lambda}$ to $\mathbb{A}_{\lambda}^{2}$ satisfying $\left.A_{\lambda}^{2}(s)\right|_{\Sigma}=B_{\lambda}^{2}(s)$. Step 3 follows since, by continuity, the claim holds for $\tau=0$ if and only if it holds for $\tau=2$.

Step 4 It suffices to assume that $v_{\lambda}=v$ is independent of $\lambda$ and there exists a neighbourhood $N \subset Y$ of $\partial Y$ such that $\left.v\right|_{N}=11,\left.A_{\lambda}(s)\right|_{N}=0,\left.\Phi_{\lambda}(s)\right|_{N}=0$, and $\left.u(s)\right|_{N}=\mathbb{1}$.

By Step 3 we can assume $v_{\lambda}=11,\left.u\right|_{S^{1} \times \Sigma}=1$, and $\left.A_{\lambda}(s)\right|_{\Sigma}=w_{\lambda}(s)^{-1} \mathrm{~d} w_{\lambda}(s)$ for a smooth map $w:[0,1] \times S^{1} \rightarrow \mathcal{G}_{z}(\Sigma)$. By a further homotopy argument we may assume that $w$ is transversally constant near the edges of the square, $\partial_{\lambda} w_{\lambda}(s)=0$ for $\lambda \simeq 0$ and $\lambda \simeq 1$, and $\partial_{s} w_{\lambda}(s)=0$ for $s \simeq 0$ and $s \simeq 1$. Since every gauge transformation on $\Sigma$ extends to a gauge transformation on $Y$ and the same holds for families parametrized by contractible domains, there is a smooth map $[0,1]^{2} \rightarrow \mathcal{G}(Y):(\lambda, s) \mapsto u_{\lambda}(s)$ such that

$$
\left.u_{\lambda}(s)\right|_{\Sigma}=w_{\lambda}(s)^{-1} \text {. }
$$

This map can be chosen such that $\partial_{\lambda} u_{\lambda}(s)=0$ for $\lambda \simeq 0$ and $\lambda \simeq 1$, and $\partial_{s} u_{\lambda}(s)=0$ for $s \simeq 0$ and $s \simeq 1$. Moreover, we can achieve $\lambda$-independence of $v_{\lambda}^{\prime}:=u_{\lambda}(0)^{-1} u_{\lambda}(1)$. To see this, note that $\left.v_{\lambda}^{\prime}\right|_{\Sigma}=\mathbb{1}$ and there is a $\delta>0$ such that $\partial_{\lambda} v_{\lambda}^{\prime}=0$ for $\lambda \notin(\delta, 1-\delta)$. Let $\beta:[0,1] \rightarrow[0,1]$ be a smooth monotone cutoff function such that $\beta(\lambda)=\lambda$ for $\lambda \in[\delta, 1-\delta], \beta \equiv 0$ for $\lambda \simeq 0$, and $\beta \equiv 1$ for $\lambda \simeq 1$. Now we can replace $u_{\lambda}(s)$ by $u_{\lambda}(s)\left(v_{\beta(s) \beta(\lambda)}^{\prime}\right)^{-1}$. The resulting map $(\lambda, s) \mapsto u_{\lambda}(s)$ satisfies $u_{\lambda}(1)=u_{\lambda}(0) v^{\prime}$ with $v^{\prime}$ independent of $\lambda$, as claimed. Hence it extends to $[0,1] \times \mathbb{R}$ such that $v^{\prime}=$ $u_{\lambda}(s)^{-1} u_{\lambda}(s+1)$ for all $\lambda$ and $s$. Define

$$
\mathbb{A}_{\lambda}^{\prime}:=u_{\lambda}^{*} \mathbb{A}_{\lambda} \in \mathcal{A}\left(P_{v^{\prime}}, \mathcal{L}\right), \quad u^{\prime}:=u_{0}^{-1} u u_{1} .
$$

Then $\left.v^{\prime}\right|_{\Sigma}=11,\left.A_{\lambda}^{\prime}\right|_{\Sigma} \equiv 0,\left.u^{\prime}\right|_{\Sigma} \equiv \mathbb{1}$, and $u^{\prime *}\left(v^{\prime}, \mathbb{A}_{0}^{\prime}\right)=\left(v^{\prime}, \mathbb{A}_{1}^{\prime}\right)$. Moreover $u_{\lambda}$ is a morphism from $\left(11, \mathbb{A}_{\lambda}\right)$ to $\left(v^{\prime}, \mathbb{A}_{\lambda}^{\prime}\right)$ for every $\lambda$. This gives a commuting diagram



There is a second diagram where the horizontal arrows are induced by the paths $\lambda \mapsto$ $\left(\mathbb{1 l}, \mathbb{A}_{\lambda}\right)$ and $\lambda \mapsto\left(v^{\prime}, \mathbb{A}_{\lambda}^{\prime}\right)=u_{\lambda}^{*}\left(\mathbb{1 l}, \mathbb{A}_{\lambda}\right)$. That this second diagram commutes as well follows from a homotopy argument; namely the space of smooth maps $[0,1]^{2} \rightarrow \mathcal{G}(Y)$ : $(s, \lambda) \mapsto u_{\lambda}(s)$ is connected and the diagram obviously commutes when $u_{\lambda}(s) \equiv \mathbb{1}$. 
This shows that the claim holds for $\left(u, \mathbb{1}, \mathbb{A}_{\lambda}\right)$ if and only if it holds for $\left(u^{\prime}, v^{\prime}, \mathbb{A}_{\lambda}^{\prime}\right)$. Hence Step 4 follows from a further homotopy argument (to achieve the relevant boundary conditions and vanishing of $\Phi$ in a neighbourhood of $\partial Y$ ).

Step 5 We prove the claim.

By Step 4, we may assume that $v_{\lambda}=v$ and there exists a neighbourhood $N \subset Y$ of $\partial Y$ such that $\left.v\right|_{N}=\mathbb{1},\left.A_{\lambda}(s)\right|_{N}=0,\left.\Phi_{\lambda}(s)\right|_{N}=0$, and $\left.u(s)\right|_{N}=\mathbb{1}$. We shall argue as in the proof of (ii), namely choose a handle body $Y^{\prime}$ with $\partial Y^{\prime}=\bar{\Sigma}$ and transfer the problem to the closed 3-manifold $\tilde{Y}:=Y \cup_{\Sigma} Y^{\prime}$.

Since the map on orientations induced by the path $\lambda \mapsto \mathbb{A}_{\lambda}$ is invariant under homotopy we may assume that the path is the straight line

$$
\mathbb{A}_{\lambda}=(1-\lambda) \mathbb{A}+\lambda u^{*} \mathbb{A},
$$

where $\mathbb{A} \in \mathcal{A}\left(P_{v}\right)$ vanishes near the boundary and $u \in \mathcal{G}\left(P_{v}\right)$ is equal to the identity near the boundary. Since $v \in \mathcal{G}(Y)$ is the identity near the boundary we can extend it to a gauge transformation $\widetilde{v} \in \mathcal{G}(\tilde{Y})$ via $\left.\widetilde{v}\right|_{Y^{\prime}}:=v^{\prime}:=\mathbb{1}$. Then $u \in \mathcal{G}\left(P_{v}\right)$ extends to a gauge transformation $\tilde{u} \in \mathcal{G}\left(P_{\widetilde{v}}\right)$ via $\left.\widetilde{u}(s)\right|_{Y^{\prime}}:=\mathbb{1}$ and $\mathbb{A}$ extends to a connection $\widetilde{\mathbb{A}} \in \mathcal{A}\left(P_{\widetilde{v}}\right)$ via $\left.\widetilde{\mathbb{A}}\right|_{S^{1} \times Y^{\prime}}:=\mathbb{A}^{\prime}=0$. As in the proof of (ii) we have three Fredholm operators $\mathcal{D}_{v, \mathbb{A}}$ on $S^{1} \times Y, \mathcal{D}_{v^{\prime}, \mathbb{A}^{\prime}}$ on $S^{1} \times Y^{\prime}$ (both with boundary conditions $\left.* \alpha\right|_{\partial Y}=0$ and $\left.\alpha\right|_{\partial Y} \in \mathrm{T}_{0} \mathcal{L}$ ), and $\mathcal{D}_{\widetilde{v}, \widetilde{\mathbb{A}}}$ on $S^{1} \times \tilde{Y}$ (without boundary conditions). We must prove that the isomorphism

$$
u^{*}: \operatorname{Or}\left(\mathcal{D}_{v, \mathbb{A}}\right) \rightarrow \operatorname{Or}\left(\mathcal{D}_{v, u^{*} \mathbb{A}}\right)
$$

agrees with the isomorphism determined by the homotopy. Since both the gauge transformation and the homotopy act trivially on $\operatorname{det}\left(\mathcal{D}_{v^{\prime}, \mathbb{A}^{\prime}}\right)$ this means that the isomorphism

$$
u^{*} \otimes \operatorname{Id}: \operatorname{Or}\left(\mathcal{D}_{v, \mathbb{A}} \times \mathcal{D}_{v^{\prime}, \mathbb{A}^{\prime}}\right) \rightarrow \operatorname{Or}\left(\mathcal{D}_{v, u^{*} \mathbb{A}} \times \mathcal{D}_{v^{\prime}, \mathbb{A}^{\prime}}\right)
$$

agrees with the homotopy isomorphism. As in the proof of (ii) we choose a family of Lagrangian subspaces connecting $\Lambda_{1}$ to $\Lambda_{2}$ to obtain two continuous families of isomorphisms (see Lemma B.16; we use the fact that the Lagrangian subspaces can be chosen as compact perturbations of $\Lambda_{0}^{\perp}$ ). For $\Lambda_{1}$ the gauge transformation induces the isomorphism (39) and for $\Lambda_{2}$ the isomorphism

$$
\tilde{u}^{*}: \operatorname{Or}\left(\mathcal{D}_{\widetilde{v}, \widetilde{\mathbb{A}}}\right) \rightarrow \operatorname{Or}\left(\mathcal{D}_{\widetilde{v}, \widetilde{u}^{*} \widetilde{\mathbb{A}}}\right)
$$

and similarly for the homotopy induced isomorphisms. For $\Lambda_{2}$ both isomorphisms agree by the standard theory for self-duality operators on closed 4-manifolds (see Donaldson [9]). Hence they agree for $\Lambda_{1}$. This proves the claim and the theorem. 
Lemma 4.8 Let $X$ be a manifold and $[0,1] \times X \rightarrow \mathcal{G}(Y):(\tau, x) \mapsto v_{x}^{\tau}$ be a smooth map. Then there is a smooth map

$$
[0,1] \times X \times \mathbb{R} \rightarrow \mathcal{G}(Y):(\tau, x, s) \mapsto u_{x}^{\tau}(s)
$$

such that

$$
v_{x}^{\tau}=u_{x}^{\tau}(s)^{-1} v_{x}^{0} u_{x}^{\tau}(s+1), \quad u_{x}^{\tau}(0)=11
$$

Proof Choose a cutoff function $\beta:[0,1] \rightarrow[0,1]$ such that $\beta(s)=0$ for $s \simeq 0$ and $\beta(s)=1$ for $s \simeq 1$. Define

$$
u_{x}^{\tau}(s):=\left(v_{x}^{0}\right)^{-1} v_{x}^{\beta(s) \tau}, \quad 0 \leq s \leq 1 .
$$

Then $u_{x}^{\tau}(s)=\mathbb{1 l}$ for $s \simeq 0$ and $u_{x}^{\tau}(s)=\left(v_{x}^{0}\right)^{-1} v_{x}^{\tau}$ for $s \simeq 1$. Hence $u_{x}^{\tau}$ extends uniquely to a smooth map from $\mathbb{R}$ to $\mathcal{G}(Y)$ that satisfies $(41)$; the extension to $(1, \infty)$ is given by $u_{x}^{\tau}(s+1):=\left(v_{x}^{0}\right)^{-1} u_{x}^{\tau}(s) v_{x}^{\tau}$ and the extension to $(-\infty, 0)$ by $u_{x}^{\tau}(s-1):=$ $v_{x}^{0} u_{x}^{\tau}(s)\left(v_{x}^{\tau}\right)^{-1}$, in both cases for $s>0$. Moreover, the resulting map $[0,1] \times X \times \mathbb{R} \rightarrow$ $\mathcal{G}(Y)$ is smooth in all variables.

\section{Exponential decay}

Let $Y$ be a compact oriented 3-manifold with boundary $\partial Y=\Sigma$ and let $\mathcal{L} \subset \mathcal{A}(\Sigma)$ be a gauge invariant, monotone Lagrangian submanifold satisfying (L1)-(L2). (Actually this section only requires the compactness of $\mathcal{L} / \mathcal{G}_{z}(\Sigma)$ from (L2).) We fix a perturbation $X_{f}: \mathcal{A}(Y) \rightarrow \Omega^{2}(Y, \mathfrak{g})$ as in Section 2. The purpose of this section is to establish the exponential decay for finite energy solutions in the following two Theorems. The unperturbed Yang-Mills energy of a connection $\mathbb{A} \in \mathcal{A}(\mathbb{R} \times Y)$ is $\frac{1}{2} \int\left|F_{\mathbb{A}}\right|^{2}$. In the presence of a holonomy perturbation the gauge invariant energy of $\mathbb{A}=A+\Phi \mathrm{d} s$ is

$$
E_{f}(\mathbb{A})=\frac{1}{2} \int_{\mathbb{R} \times Y}\left|F_{\mathbb{A}}+X_{f}(\mathbb{A})\right|^{2}=\frac{1}{2} \int_{\mathbb{R} \times Y}\left(\left|\partial_{s} A-\mathrm{d}_{A} \Phi\right|^{2}+\left|F_{A}+X_{f}(A)\right|^{2}\right) .
$$

An anti-self-dual connection in temporal gauge satisfies $\partial_{s} A+*\left(F_{A}+X_{f}(A)\right)=0$ and $\Phi=0$ and the energy simplifies to $E_{f}(\mathbb{A})=\int_{\mathbb{R} \times Y}\left|\partial_{S} A\right|^{2}$.

Theorem 5.1 Suppose that every critical point of the perturbed Chern-Simons functional $\mathcal{C S}_{\mathcal{L}}+h_{f}$ is nondegenerate. Then there is a constant $\delta>0$ such that the following holds. If $A:[0, \infty) \rightarrow \mathcal{A}(Y)$ is a smooth solution of

$$
\partial_{s} A+*\left(F_{A}+X_{f}(A)\right)=0,\left.\quad A(s)\right|_{\partial Y} \in \mathcal{L},
$$


satisfying

$$
\int_{0}^{\infty} \int_{Y}\left|\partial_{S} A\right|^{p} \operatorname{dvol}_{Y} \mathrm{~d} s<\infty, \quad p \geq 2,
$$

then there is a connection $A_{\infty} \in \mathcal{A}(Y, \mathcal{L})$ such that $F_{A_{\infty}}+X_{f}\left(A_{\infty}\right)=0$ and $A(s)$ converges to $A$ as $s \rightarrow \infty$. Moreover, there are constants $C_{0}, C_{1}, C_{2}, \ldots$ such that

$$
\left\|A-A_{\infty}\right\|_{\mathcal{C}^{k}([s-1, s+1] \times Y)} \leq C_{k} e^{-\delta s}
$$

for every $s \geq 1$ and every integer $k \geq 0$.

Remark 5.2 Let $X$ be a compact Riemannian manifold with boundary. We shall need gauge invariant Sobolev norms on the spaces $\Omega^{\ell}(X, \mathfrak{g})$ depending on a connection $\mathbb{A} \in \mathcal{A}(X)$. For $p \geq 1$ and an integer $k \geq 0$ we define

$$
\|\alpha\|_{W^{k, p}, \mathbb{A}}:=\left(\sum_{j=0}^{k} \int_{X}\left|\nabla_{\mathbb{A}}^{j} \alpha\right|^{p}\right)^{1 / p}
$$

for $\alpha \in \Omega^{\ell}(Y, \mathfrak{g})$, where $\nabla_{\mathbb{A}}^{j} \alpha$ denotes the $j$-th covariant derivative of $\alpha$ twisted by $\mathbb{A}$. For $p=\infty$ we define

$$
\|\alpha\|_{W^{k, \infty, \mathbb{A}}}:=\|\alpha\|_{\mathcal{C}^{k}, \mathbb{A}}:=\max _{0 \leq j \leq k} \sup _{X}\left|\nabla_{\mathbb{A}}^{j} \alpha\right| .
$$

These norms are gauge invariant in the sense that

$$
\left\|u^{-1} \alpha u\right\|_{W^{k, p}, u^{*} \mathbb{A}}=\|\alpha\|_{W^{k, p}, \mathbb{A}}
$$

for every gauge transformation $u \in \mathcal{G}(X)$. In particular, for $k=0$ the $L^{p}$-norms are gauge invariant and do not depend on the connection $\mathbb{A}$.

Theorem 5.3 Suppose that every critical point of the perturbed Chern-Simons functional is nondegenerate. Then, for every $p>1$, there are positive constants $\varepsilon, \delta$, $C_{0}, C_{1}, \ldots$ such that the following holds for every $T \geq 1$. If $A:[-T, T] \rightarrow \mathcal{A}(Y)$ is a smooth solution of (42) satisfying

$$
\int_{-T}^{T} \int_{Y}\left|\partial_{S} A\right|^{2} \mathrm{dvol}_{Y} \mathrm{~d} s<\varepsilon
$$

then, for every $s \in[0, T-1]$ and every integer $k \geq 0$,

$$
\left\|\partial_{s} A\right\|_{\mathcal{C}^{k}([-s, s] \times Y), \mathbb{A}} \leq C_{k} e^{-\delta(T-s)}\left\|\partial_{s} A\right\|_{L^{2}(([-T, 1-T] \cup[T-1, T]) \times Y)},
$$


where $\mathbb{A} \in \mathcal{A}([-T, T] \times Y)$ is the connection associated to the path $A$. Moreover, there is a connection $A_{0} \in \mathcal{A}(Y, \mathcal{L})$ with $F_{A_{0}}+X_{f}\left(A_{0}\right)=0$ such that

$$
\begin{aligned}
& \left\|A-A_{0}\right\|_{\mathcal{C}^{0}([-s, s] \times Y)}+\left\|A-A_{0}\right\|_{W^{1, p}([-s, s] \times Y), A_{0}} \\
& \quad \leq C_{0} e^{-\delta(T-s)}\left\|\partial_{S} A\right\|_{L^{2}(([-T, 1-T] \cup[T-1, T]) \times Y)}
\end{aligned}
$$

for every $s \in[0, T-1]$.

The proofs of these results will be given below. Theorem 5.1 guarantees the existence of a limit for each finite energy solution of (42), however, the constants in the exponential decay estimate depend on the solution. With the help of Theorem 5.3 one can show that these constants can be chosen independent of the solution of (42) and depend only on the limit $A_{\infty}$. This will be important for the gluing analysis.

Corollary 5.4 Let $A_{\infty}$ be a nondegenerate critical point of the perturbed ChernSimons functional $\mathcal{C} \mathcal{S}_{\mathcal{L}}+h_{f}$. Then there are positive constant $\delta, \varepsilon, C_{0}, C_{1}, \ldots$ such that the following holds. If $A:[0, \infty) \rightarrow \mathcal{A}(Y)$ is a smooth solution of (42) satisfying

$$
\int_{0}^{\infty} \int_{Y}\left|\partial_{s} A\right|^{2} \operatorname{dvol}_{Y} \mathrm{~d} s<\varepsilon, \quad \lim _{s \rightarrow \infty} A(s)=A_{\infty},
$$

then $\quad\left\|A-A_{\infty}\right\|_{\mathcal{C}^{k}([s, \infty) \times Y)} \leq C_{k} e^{-\delta s}\left\|\partial_{s} A\right\|_{L^{2}([0, \infty) \times Y)}$

for every $s \geq 1$ and every integer $k \geq 0$.

Proof Let $\delta, \varepsilon, C_{k}^{\prime}$ be the constants of Theorem 5.3. Then

$$
\left\|\partial_{s} A\right\|_{\mathcal{C}^{k}([s, \infty) \times Y), \mathbb{A}} \leq C_{k}^{\prime} e^{-\delta s}\left\|\partial_{s} A\right\|_{L^{2}([0, \infty) \times Y)}
$$

for $k=0,1,2, \ldots$ and $s \geq 1$. For $k=0$ the desired estimate follows by integrating from $s$ to $\infty$ because the $\mathcal{C}^{0}$-norm is independent of the reference connection $\mathbb{A}$. Now argue by induction. If the result has been established for any $k$ then there is a constant $c_{k}$, depending on $C_{k}$, such that

$$
\|\alpha\|_{\mathcal{C}^{k+1}([s, \infty) \times Y)} \leq c_{k}\|\alpha\|_{\mathcal{C}^{k+1}([s, \infty) \times Y), \mathbb{A}}
$$

for every $\alpha$ : $[1, \infty) \rightarrow \Omega^{1}(Y, \mathfrak{g})$. Applying this to $\alpha=\partial_{s} A$ we obtain

$$
\left\|\partial_{s} A\right\|_{\mathcal{C}^{k+1}([s, \infty) \times Y)} \leq c_{k} C_{k+1}^{\prime} e^{-\delta s}\left\|\partial_{s} A\right\|_{L^{2}([0, \infty) \times Y)}
$$

and the required $\mathcal{C}^{k+1}$-estimate follows again by integrating from $s$ to $\infty$. This proves the corollary. 
The proof of Theorem 5.1 and Theorem 5.3 is based on the following three lemmas concerning solutions on a long cylinder with little energy. We show that such solutions are uniformly close to a critical point and establish uniform estimates for the Hessian and the linearized operator.

Lemma 5.5 For every $\kappa>0, \rho>0$, and $p>1$ there is an $\varepsilon>0$ such that the following holds. If $A:[-\rho, \rho] \rightarrow \mathcal{A}(Y)$ is a solution of (42) that satisfies

$$
\int_{-\rho}^{\rho} \int_{Y}\left|\partial_{S} A\right|^{2} \mathrm{dvol}_{Y} \mathrm{~d} s<\varepsilon
$$

then there is a connection $A_{\infty} \in \mathcal{A}(Y, \mathcal{L})$ with $F_{A_{\infty}}+X_{f}\left(A_{\infty}\right)=0$ such that

$$
\left\|A(0)-A_{\infty}\right\|_{W^{1, p}(Y), A_{\infty}}+\left\|A(0)-A_{\infty}\right\|_{L^{\infty}(Y)}+\left\|\partial_{s} A(0)\right\|_{L^{\infty}(Y)}<\kappa .
$$

Proof Assume by contradiction that this is wrong. Then there exist constants $\kappa>0$, $\rho>0$, and $p>1$ and a sequence $A_{v}:[-\rho, \rho] \rightarrow \mathcal{A}(Y)$ of solutions of (42) such that

$$
\lim _{\nu \rightarrow \infty} \int_{-\rho}^{\rho} \int_{Y}\left|\partial_{s} A_{\nu}\right|^{2} \mathrm{dvol}_{Y} \mathrm{~d} s=0
$$

but (46) fails. Let $\mathbb{A}_{v} \in \mathcal{A}([-\rho, \rho] \times Y)$ denote the connection in temporal gauge associated to the path $A_{v}$. Then $F_{\mathbb{A}_{v}}+X_{f}\left(\mathbb{A}_{v}\right)$ converges to zero in the $L^{2}$-norm, by (47) and (42). Now it follows from the energy quantization of Wehrheim [36, Theorems 1.2, 2.1] (for general Lagrangians see Mrowka and Wehrheim [23], and for the perturbed version see Theorem D.4) that $\mathbb{A}_{v}$ satisfies an $L^{\infty}$-bound on the curvature. Hence, by [35, Theorem B] and Theorem D.4, there is a subsequence (still denoted by $\left.\mathbb{A}_{v}\right)$ and a sequence of gauge transformations $u_{v} \in \mathcal{G}([-\rho / 2, \rho / 2] \times Y)$ such that $u_{v}^{*} \mathbb{A}_{v}$ converges to $\mathbb{A}_{\infty}=A_{\infty}(s)+\Phi_{\infty}(s) \mathrm{d} s \in \mathcal{A}([-\rho / 2, \rho / 2] \times Y)$ in the $\mathcal{C}^{\infty}$-topology. By (42) and (47) the limit connection satisfies

$$
\partial_{s} A_{\infty}(s)-\mathrm{d}_{A_{\infty}(s)} \Phi_{\infty}(s)=0, \quad F_{A_{\infty}(s)}+X_{f}\left(A_{\infty}(s)\right)=0,\left.\quad A_{\infty}(s)\right|_{\Sigma} \in \mathcal{L}
$$

for every $s \in[-\rho / 2, \rho / 2]$. After modifying the gauge transformations $u_{v}$ we may assume in addition that $\Phi_{\infty}(s)=0$ and $A_{\infty}(s)=A_{\infty}$ is independent of $s$. It then follows that $u_{v}^{-1} \partial_{s} u_{v}$ converges to zero in the $\mathcal{C}^{\infty}$-topology. So after a further modification we can assume that the $u_{v}(s)=u_{v}$ is independent of $s$, and so the convergent connections $u_{v}^{*} \mathbb{A}_{v}$ are in temporal gauge, given by the paths $[-\rho / 2, \rho / 2] \rightarrow \mathcal{A}(Y): s \mapsto u_{v}^{*} A_{v}(s)$. Hence

$$
\begin{aligned}
& \lim _{\nu \rightarrow \infty}\left\|A_{\nu}(0)-\left(u_{v}^{-1}\right)^{*} A_{\infty}\right\|_{W^{1, p}(Y), u_{\nu}^{-1 *} A_{\infty}}=\lim _{\nu \rightarrow \infty}\left\|\left(u_{\nu}^{*} \mathbb{A}_{\nu}-\mathbb{A}_{\infty}\right)(0)\right\|_{W^{1, p}, A_{\infty}}=0, \\
& \lim _{\nu \rightarrow \infty}\left\|A_{\nu}(0)-\left(u_{\nu}^{-1}\right)^{*} A_{\infty}\right\|_{L^{\infty}(Y)}=\lim _{\nu \rightarrow \infty}\left\|\left(u_{\nu}^{*} \mathbb{A}_{\nu}-\mathbb{A}_{\infty}\right)(0)\right\|_{L^{\infty}(Y)}=0, \\
& \lim _{\nu \rightarrow \infty}\left\|\partial_{S} A_{\nu}(0)\right\|_{L^{\infty}(Y)}=\lim _{\nu \rightarrow \infty}\left\|\partial_{S}\left(u_{v}^{*} \mathbb{A}_{\nu}\right)(0)\right\|_{L^{\infty}(Y)}=\left\|\partial_{s} A_{\infty}\right\|_{L^{\infty}(Y)}=0 .
\end{aligned}
$$


This contradicts the assumption that (46) fails, and thus proves the lemma.

Lemma 5.6 Suppose that every critical point of the perturbed Chern-Simons functional $\mathcal{C} \mathcal{S}_{\mathcal{L}}+h_{f}$ is nondegenerate. Then, for every $\rho>0$, there are positive constants $c_{0}$ and $\varepsilon$ with the following significance. If $A:[-\rho, \rho] \rightarrow \mathcal{A}(Y)$ is a solution of (42) such that

$$
\int_{-\rho}^{\rho} \int_{Y}\left|\partial_{s} A\right|^{2} \mathrm{dvol}_{Y} \mathrm{~d} s<\varepsilon
$$

then for every $\alpha \in \Omega_{A(0)}^{1}(Y, \mathfrak{g})$

$$
\|\alpha\|_{L^{6}(Y)}+\|\alpha\|_{L^{4}(\partial Y)} \leq c_{0}\left(\left\|\mathrm{~d}_{A(0)} \alpha+\mathrm{d} X_{f}(A(0)) \alpha\right\|_{L^{2}(Y)}+\left\|\mathrm{d}_{A(0)}^{*} \alpha\right\|_{L^{2}(Y)}\right) .
$$

Proof Assume by contradiction that this is wrong. Then there is a constant $\rho>0$, a sequence $A_{v}:[-\rho, \rho] \rightarrow \mathcal{A}(Y)$ of solutions of (42) with (47), and a sequence $\alpha_{v} \in$ $\Omega_{A_{\nu}(0)}^{1}(Y, \mathfrak{g})$ such that

$$
\frac{\left\|\alpha_{v}\right\|_{L^{6}(Y)}+\left\|\alpha_{\nu}\right\|_{L^{4}(\partial Y)}}{\left\|\mathrm{d}_{A_{\nu}(0)} \alpha_{v}+\mathrm{d} X_{f}\left(A_{v}(0)\right) \alpha_{v}\right\|_{L^{2}(Y)}+\left\|\mathrm{d}_{A_{\nu}(0)}^{*} \alpha_{\nu}\right\|_{L^{2}(Y)}} \underset{\nu \rightarrow \infty}{\longrightarrow} \infty .
$$

Arguing as in the proof of Lemma 5.5 we find a subsequence, still denoted by $A_{v}$, and a sequence of gauge transformations $u_{v} \in \mathcal{G}(Y)$ such that $u_{v}^{*} A_{v}(0)$ converges in the $\mathcal{C}^{\infty}$-topology to a connection $A_{\infty} \in \mathcal{A}(Y, \mathcal{L})$ that satisfies $F_{A_{\infty}}+X_{f}\left(A_{\infty}\right)=0$. By assumption $A_{\infty}$ is nondegenerate, so by Corollary 3.6 there is a constant $C$ such that

$$
\|(\alpha, 0)\|_{W^{1,2}(Y)} \leq C\left\|\mathcal{H}_{A_{\infty}}(\alpha, 0)\right\|_{L^{2}(Y)}
$$

for every $(\alpha, 0) \in \operatorname{dom} \mathcal{H}_{A_{\infty}}$. By Theorem E.2 this estimate is stable under $\mathcal{C}^{1}-$ small perturbations of $A_{\infty}$, and by gauge invariance it continues to hold with $A_{\infty}$ replaced by $A_{v}(0)$. Precisely, let $\mathcal{U} \subset \mathcal{A}(Y, \mathcal{L})$ be a neighbourhood of $A_{\infty}$ and $\left\{Q_{A}\right\}_{A \in \mathcal{U}}$ be an operator family that satisfies the requirements of Theorem E.2. Then $u_{v}^{*} A_{v}(0) \in \mathcal{U}$ for large $v$ add the isomorphisms $Q_{v}:=Q_{u_{v}^{*} A_{v}(0)} \times \operatorname{Id}$ from dom $\mathcal{H}_{A_{\infty}}$ to dom $\mathcal{H}_{u_{\nu}^{*} A_{\nu}(0)}$ converge to $Q_{A_{\infty}} \times \operatorname{Id}=\operatorname{Id}$ in both $\mathcal{L}\left(W^{1,2}\right)$ and $\mathcal{L}\left(L^{2}\right)$; so the sequence $Q_{v}^{-1} \mathcal{H}_{u_{v}^{*} A_{v}(0)} Q_{v}$ has the constant domain dom $\mathcal{H}_{A_{\infty}}$, and it converges to $\mathcal{H}_{A_{\infty}}$ in the operator norm on $\mathcal{L}\left(W^{1,2}, L^{2}\right)$. Hence, for large $v$, we can replace $\mathcal{H}_{A_{\infty}}$ by $Q_{v}^{-1} \mathcal{H}_{u_{v}^{*} A_{v}(0)} Q_{v}$ in (49) to obtain estimates with a uniform constant $C$. Since $Q_{v}$ converges to the identity in the relevant operator norms we obtain the following estimate with uniform constants $C_{i}$ but varying domain:

$$
\|\alpha\|_{L^{6}(Y)}+\|\alpha\|_{L^{4}(\partial Y)} \leq C_{1}\|(\alpha, 0)\|_{W^{1,2}(Y)} \leq C_{2}\left\|\mathcal{H}_{u_{v}^{*} A_{v}(0)}(\alpha, 0)\right\|_{L^{2}(Y)}
$$

for every $(\alpha, 0) \in \operatorname{dom} \mathcal{H}_{u_{\nu}^{*}} A_{\nu}(0)$. Here we used the Sobolev embedding $W^{1,2}(Y) \hookrightarrow$ $L^{6}(Y)$ and the trace theorem $W^{1,2}(Y) \hookrightarrow L^{4}(\partial Y)$. Since $\mathrm{T}_{u^{*}} \mathcal{L}=u^{-1}\left(\mathrm{~T}_{A} \mathcal{L}\right) u$ we 
can apply the last estimate to $\left(u_{v}^{-1} \alpha_{v} u_{v}, 0\right) \in \operatorname{dom} \mathcal{H}_{u_{\nu}^{*} A_{v}(0)}$. Since the norms on the left and right hand side are all gauge invariant the resulting inequality contradicts (48). This proves the lemma.

Lemma 5.7 Suppose that every critical point of the perturbed Chern-Simons functional $\mathcal{C S}_{\mathcal{L}}+h_{f}$ is nondegenerate. Then, for every $\rho>\rho^{\prime}>0$, there are positive constants $c_{0}, c_{1}, \ldots$ and $\varepsilon$ with the following significance. If $A:[-\rho, \rho] \rightarrow \mathcal{A}(Y)$ is a solution of (42) such that

$$
\int_{-\rho}^{\rho} \int_{Y}\left|\partial_{S} A\right|^{2} \mathrm{dvol}_{Y} \mathrm{~d} s<\varepsilon
$$

then, for every smooth path $[-\rho, \rho] \rightarrow \Omega^{1}(Y, \mathfrak{g}) \times \Omega^{0}(Y, \mathfrak{g}): s \mapsto(\alpha(s), \varphi(s))$ satisfying $\alpha(s) \in \Omega_{A(s)}^{1}(Y, \mathfrak{g})$ and every integer $k \geq 0$, we have

$$
\begin{aligned}
\|(\alpha, \varphi)\|_{\mathcal{C}^{k}\left(\left[-\rho^{\prime}, \rho^{\prime}\right] \times Y\right), \mathbb{A}} & \\
& \leq c_{k}\left(\left\|\mathcal{D}_{\mathbb{A}}(\alpha, \varphi)\right\|_{W^{k+2,2}([-\rho, \rho] \times Y), \mathbb{A}}+\|(\alpha, \varphi)\|_{L^{2}([-\rho, \rho] \times Y)}\right) .
\end{aligned}
$$

Proof If this is wrong, then there exist constants $k \geq 0, \rho>\rho^{\prime}>0$ and a sequence $A_{v}:[-\rho, \rho] \rightarrow \mathcal{A}(Y)$ of solutions of (42) with (47), for which the constant in the estimate blows up. As in the proof of Lemma 5.5 we find a subsequence of the connections on $[-\rho, \rho] \times Y$, still denoted by $\mathbb{A}_{v}$, and gauge transformations $u_{v} \in \mathcal{G}(Y)$ such that $u_{v}^{*} \mathbb{A}_{v}$ converges in the $\mathcal{C}^{\infty}$-topology on $[-\rho / 2, \rho / 2] \times Y$ to a constant connection $\mathbb{A}_{\infty}=A_{\infty} \in \mathcal{A}(Y, \mathcal{L})$. Now by Theorem 3.11 and the Sobolev embedding theorem, and with the norms of Remark 5.2, there is a constant $C$ such that for every $(\alpha, \varphi)$ satisfying $\alpha(s) \in \Omega_{A_{\infty}}^{1}(Y, \mathfrak{g})$,

$$
\begin{aligned}
& \|(\alpha, \varphi)\|_{\mathcal{C}^{k}\left(\left[-\rho^{\prime}, \rho^{\prime}\right] \times Y\right), \mathbb{A}_{\infty}} \\
& \quad \leq C\left(\left\|\mathcal{D}_{\mathbb{A}_{\infty}}(\alpha, \varphi)\right\|_{W^{k+2,2}([-\rho, \rho] \times Y), \mathbb{A}_{\infty}}+\|(\alpha, \varphi)\|_{L^{2}([-\rho, \rho] \times Y), \mathbb{A}_{\infty}}\right) .
\end{aligned}
$$

The same argument as in the proof of Lemma 5.6 (with the sequence of operators $\left.Q_{\nu}(s):=Q_{u_{\nu}^{*} A_{\nu}(s)} \times \mathrm{Id}\right)$ shows that this estimate continues to hold with $\mathbb{A}_{\infty}$ replaced by $u_{v}^{*} \mathbb{A}_{v}$. Note that $\mathcal{D}_{u_{v}^{*} \mathbb{A}_{v}} u_{v}^{-1}\left(\alpha_{v}, \varphi_{v}\right) u_{v}=u_{v}^{-1}\left(\mathcal{D}_{\mathbb{A}_{v}}\left(\alpha_{v}, \varphi_{v}\right)\right) u_{v}$. So since the norms are gauge invariant, the above estimate also holds with $\mathbb{A}_{\infty}$ replaced by $\mathbb{A}_{v}$, which contradicts the choice of $A_{v}$ and thus proves the lemma.

Proof of Theorem 5.1 The proof has three steps. 
Step 1 There is a uniform constant $\delta>0$ (independent of the solution $A$ ) and a constant $C$ (which depends on $A$ ) such that

$$
\left\|\partial_{s} A(s)\right\|_{L^{2}(Y)} \leq C e^{-\delta s} \quad \text { for } s \geq 0 .
$$

Define $\quad g(s):=\frac{1}{2} \int_{Y}\left|\partial_{s} A\right|^{2}=\frac{1}{2} \int_{Y}\left|F_{A}+X_{f}(A)\right|^{2}$.

Then $\quad g^{\prime}(s)=\int_{Y}\left\langle\left(\mathrm{~d}_{A} \partial_{s} A+\mathrm{d} X_{f}(A) \partial_{s} A\right) \wedge *\left(F_{A}+X_{f}(A)\right)\right\rangle$,

and hence

$$
\begin{aligned}
& g^{\prime \prime}(s)=\int_{Y}\left|\mathrm{~d}_{A} \partial_{S} A+\mathrm{d} X_{f}(A) \partial_{S} A\right|^{2}-\int_{Y}\left\langle\left(\left[\partial_{S} A \wedge \partial_{S} A\right]+\mathrm{d}_{A} \partial_{S}^{2} A\right) \wedge \partial_{S} A\right\rangle \\
& -\int_{Y}\left\langle\left(\mathrm{~d}^{2} X_{f}(A)\left(\partial_{s} A, \partial_{s} A\right)+\mathrm{d} X_{f}(A) \partial_{s}^{2} A\right) \wedge \partial_{s} A\right\rangle \\
& =\int_{Y}\left|\mathrm{~d}_{A} \partial_{S} A+\mathrm{d} X_{f}(A) \partial_{S} A\right|^{2}-\int_{Y}\left\langle\partial_{S}^{2} A \wedge\left(\mathrm{d}_{A} \partial_{S} A+\mathrm{d} X_{f}(A) \partial_{S} A\right)\right\rangle \\
& -\int_{Y}\left\langle\left(\left[\partial_{s} A \wedge \partial_{s} A\right]+\mathrm{d}^{2} X_{f}(A)\left(\partial_{s} A, \partial_{s} A\right)\right) \wedge \partial_{s} A\right\rangle-\int_{\Sigma}\left\langle\partial_{s}^{2} A \wedge \partial_{s} A\right\rangle \\
& \geq 2\left\|\mathrm{~d}_{A} \partial_{s} A+\mathrm{d} X_{f}(A) \partial_{s} A\right\|_{L^{2}(Y)}^{2} \\
& -c_{1}\left\|\partial_{S} A\right\|_{L^{\infty}(Y)}\left\|\partial_{S} A\right\|_{L^{2}(Y)}^{2}-c_{1}\left\|\partial_{S} A\right\|_{L^{3}(\partial Y)}^{3} \\
& \geq\left(4 \delta^{2}-c_{2}\left\|\partial_{S} A\right\|_{L^{\infty}(Y)}\right)\left(\left\|\partial_{S} A\right\|_{L^{2}(Y)}^{2}+\left\|\partial_{S} A\right\|_{L^{3}(\partial Y)}^{2}\right) \\
& \geq 2 \delta^{2}\left\|\partial_{S} A\right\|_{L^{2}(Y)}^{2}
\end{aligned}
$$

for uniform constants $c_{i}$ and $\delta>0$ and $s$ sufficiently large. Here we used (42). In the first inequality the term $\int_{\Sigma}\left\langle\partial_{s}^{2} A \wedge \partial_{s} A\right\rangle$ is controlled by $\left\|\partial_{s} A\right\|_{L^{3}(\partial Y)}^{3}$; see Wehrheim [36, Lemma 2.3; 23] for general Lagrangian submanifolds. The first inequality also uses the estimate on $\mathrm{d}^{2} X_{f}(A)$ from Proposition D.1 (v). For the second inequality note that every solution of (42) satisfies $\partial_{s} A(s) \in \mathrm{T}_{A(s)} \mathcal{L}$ and

$$
\begin{gathered}
\left.* \partial_{S} A\right|_{\partial Y}=-\left.\left(F_{A}+X_{f}(A)\right)\right|_{\partial Y}=0, \\
\mathrm{~d}_{A}^{*} \partial_{S} A=* \mathrm{~d}_{A}\left(F_{A}+X_{f}(A)\right)=0 .
\end{gathered}
$$

These identities use (10) and the Bianchi identity as well as the facts that the perturbation vanishes near $\partial Y$ and that the Lagrangian submanifold $\mathcal{L}$ is contained in the flat connections on $\partial Y$. Now we can apply Lemma 5.6 to the paths $[-1,1] \rightarrow \mathcal{A}(Y): \sigma \mapsto$ 
$A(s+\sigma)$ (whose derivative is $L^{2}$-small due to the finite $L^{p}$-energy of the path) and to the 1 -forms $\alpha=\partial_{s} A(s) \in \Omega_{A(s)}^{1}(Y, \mathfrak{g})$, for sufficiently large $s \geq 0$ to obtain

$$
\left\|\partial_{S} A\right\|_{L^{2}(Y)}^{2}+\left\|\partial_{S} A\right\|_{L^{3}(\partial Y)}^{2} \leq\left(2 \delta^{2}\right)^{-1}\left\|\mathrm{~d}_{A} \partial_{S} A+\mathrm{d} X_{f}(A) \partial_{S} A\right\|_{L^{2}(Y)}^{2} .
$$

Here we have chosen $\left(2 \delta^{2}\right)^{-1}=\left(c_{0} c\right)^{2}$ with the constant $c_{0}$ from Lemma 5.6 and a further Sobolev constant $c$, so $\delta>0$ is independent of the solution $A$. The last inequality in the estimate of $g^{\prime \prime}$ is due to $\left\|\partial_{s} A(s)\right\|_{L^{\infty}(Y)} \leq 2 \delta^{2} c_{2}^{-1}$ for $s$ sufficiently large. This follows from Lemma 5.5 applied to the paths $[-1,1] \rightarrow \mathcal{A}(Y): \sigma \mapsto A(s+\sigma)$. So we have $g^{\prime \prime}(s) \geq 4 \delta^{2} g(s)$ for $s$ sufficiently large. This implies the assertion of Step 1, ie $g(s) \leq C^{2} e^{-2 \delta s}$, by a standard argument (see eg the proof of [29, Lemma 2.11]).

Step 2 Let $\delta>0$ be the constant of Step 1 and $\mathbb{A} \in \mathcal{A}([0, \infty) \times Y)$ be the connection associated to the path $A$. For every integer $k \geq 0$ there is a constant $C_{k}$ such that for every $s \geq 1$

$$
\left\|\partial_{s} A\right\|_{\mathcal{C}^{k}([s-1, s+1] \times Y), \mathbb{A}} \leq C_{k} e^{-\delta s} .
$$

Fix $k \geq 0$ and consider the connections $\mathbb{A}_{\sigma} \in \mathcal{A}([-2,2] \times Y)$ given by the paths $A_{\sigma}(s):=A(\sigma+s)$. Due to the finite $L^{p}$-energy of $A$ on $[0, \infty)$ for some $p \geq 2$ these paths on $[-2,2]$ satisfy $\left\|\partial_{S} A_{\sigma}\right\|_{L^{2}([-2,2] \times Y)} \rightarrow 0$ as $\sigma \rightarrow \infty$. So by Lemma 5.7 there is a constant $c_{k}$ such that for all sufficiently large $\sigma$

$$
\begin{aligned}
\|(\alpha, \varphi)\|_{\mathcal{C}^{k}([-1,1] \times Y), \mathbb{A}_{\sigma}} & \\
& \leq c_{k}\left(\left\|\mathcal{D}_{\mathbb{A}_{\sigma}}(\alpha, \varphi)\right\|_{W^{k+2,2}([-2,2] \times Y), \mathbb{A}_{\sigma}}+\|(\alpha, \varphi)\|_{L^{2}([-2,2] \times Y)}\right)
\end{aligned}
$$

for every smooth $\varphi:[-2,2] \rightarrow \Omega^{0}(Y, \mathfrak{g})$ and $\alpha:[-2,2] \rightarrow \Omega^{1}(Y, \mathfrak{g})$ satisfying $\alpha(s) \in$ $\Omega_{A_{\sigma}(s)}^{1}(Y, \mathfrak{g})$. Now apply the estimate to the pair

$$
\alpha(s):=\partial_{s} A(\sigma+s), \quad \varphi(s):=0 .
$$

Differentiate (42) and recall (51) to see that $(\alpha, \varphi) \in \operatorname{ker} \mathcal{D}_{\mathbb{A}_{\sigma}}$ and hence

$$
\left\|\partial_{S} A\right\|_{\mathcal{C}^{k}([\sigma-1, \sigma+1] \times Y), \mathbb{A}} \leq c_{k}\left\|\partial_{S} A\right\|_{L^{2}([\sigma-2, \sigma+2] \times Y)} \leq c_{k} C(2 \delta)^{-\frac{1}{2}} e^{2 \delta} e^{-\delta \sigma} .
$$

The last inequality follows from Step 1 and proves Step 2.

Step 3 Let $\delta>0$ be the constant of Step 1. Then there is a connection $A_{\infty} \in \mathcal{A}(Y, \mathcal{L})$ such that $F_{A_{\infty}}+X_{f}\left(A_{\infty}\right)=0$ and a sequence of constants $C_{0}, C_{1}, C_{2}, \ldots$ such that

$$
\left\|A-A_{\infty}\right\|_{\mathcal{C}^{k}([s-1, s+1] \times Y)} \leq C_{k} e^{-\delta s}
$$

for every integer $k \geq 0$ and every $s \geq 1$. 
By Step 2 we have $\left\|\partial_{s} A(s)\right\|_{L^{\infty}(Y)} \leq C_{0} e^{-\delta s}$ for every $s \geq 0$. Hence the integral

$$
A_{\infty}:=A(0)+\int_{0}^{\infty} \partial_{s} A(s) \mathrm{d} s=\lim _{s \rightarrow \infty} A(s)
$$

converges in $L^{\infty}\left(Y, \mathrm{~T}^{*} Y \otimes \mathfrak{g}\right)$ and defines a $\mathcal{C}^{0}$-connection on $Y$. This directly implies $A_{\infty} \mid \Sigma \in \mathcal{L}$. Moreover, (52) holds with $k=0$. We prove by induction on $k$ that $A_{\infty}$ is a $\mathcal{C}^{k}$ connection that satisfies (52). For $k=0$ this is what we have just proved. Fix an integer $k \geq 1$ and suppose that $A_{\infty}$ is a $\mathcal{C}^{k-1}$ connection that satisfies (52) with $k$ replaced by $k-1$. Then $\mathbb{A}$ is bounded in $\mathcal{C}^{k-1}$ and so there is a constant $C$ such that

$$
\|\alpha\|_{\mathcal{C}^{\ell}([s-1, s+1] \times Y)} \leq C\|\alpha\|_{\mathcal{C}^{\ell}([s-1, s+1] \times Y), \mathbb{A}}
$$

for every $\ell \leq k, s \geq 1$, and every $\alpha \in \Omega^{1}\left([s-1, s+1] \times Y, \mathrm{~T}^{*} Y \otimes \mathfrak{g}\right)$. So it follows from Step 2 that

$$
\left\|\partial_{s} A\right\|_{\mathcal{C}^{k}([s-1, s+1] \times Y)} \leq C C_{k} e^{-\delta s} .
$$

Hence for $s_{1} \geq s_{0} \geq 0$

$$
\left\|A\left(s_{0}\right)-A\left(s_{1}\right)\right\|_{\mathcal{C}^{k}(Y)} \leq \int_{s_{0}}^{s_{1}}\left\|\partial_{s} A\right\|_{\mathcal{C}^{k}(Y)} \mathrm{d} s \leq \frac{C C_{k}}{\delta} e^{-\delta s_{0}} .
$$

This shows that $A_{\infty}$ is a $\mathcal{C}^{k}$ connection with

$$
\left\|A(s)-A_{\infty}\right\|_{\mathcal{C}^{k}(Y)} \leq \frac{C C_{k}}{\delta} e^{-\delta s}
$$

The exponential decay of $\partial_{s}^{\ell}\left(A(s)-A_{\infty}\right)=\partial_{s}^{\ell} A(s)$ in $\mathcal{C}^{k-\ell}(Y)$ for $\ell=1, \ldots, k$ follows from Step 2 and (53), so this implies (52). Moreover,

$$
F_{A_{\infty}}+X_{f}\left(A_{\infty}\right)=\lim _{s \rightarrow \infty}\left(F_{A(s)}+X_{f}(A(s))\right)=-\lim _{s \rightarrow \infty} * \partial_{s} A(s)=0 .
$$

This proves Step 3 and the lemma.

Proof of Theorem 5.3 Let $\delta>0$ be the constant of Step 1 in the proof of Theorem 5.1. We prove that there are constants $C$ and $\varepsilon>0$ such that the following holds for every $T \geq 1$. If $A:[-T, T] \rightarrow \mathcal{A}(Y)$ is a solution of (42) that satisfies (43), then it also satisfies

$$
\left\|\partial_{S} A(s)\right\|_{L^{2}(Y)} \leq C e^{-\delta(T-|s|)}\left\|\partial_{S} A\right\|_{L^{2}(([-T, 1-T] \cup[T-1, T]) \times Y)}
$$

for $|s| \leq T-1 / 2$. Let $\varepsilon>0$ be the constant of Lemma 5.6 with $\rho=\frac{1}{4}$ and assume that (43) holds with this constant $\varepsilon$. Define $f:[-T, T] \rightarrow \mathbb{R}$ by

$$
f(s):=\frac{1}{2}\left\|\partial_{s} A(s)\right\|_{L^{2}(Y)}^{2} .
$$


Then the same argument as in Step 1 in the proof of Theorem 5.1 shows that there is a constants $c_{2}$, independent of $A$, such that for $|s| \leq T-1 / 4$

$$
f^{\prime \prime}(s) \geq 2\left(4 \delta^{2}-c_{2}\left\|\partial_{s} A(s)\right\|_{L^{\infty}(Y)}\right)\left(f(s)+\left\|\partial_{s} A(s)\right\|_{L^{3}(\partial Y)}^{2}\right) .
$$

Shrinking $\varepsilon$ if necessary we may assume that $\left\|\partial_{s} A(s)\right\|_{L^{\infty}(Y)} \leq 2 \delta^{2} / c_{2}$ by Lemma 5.5 with $\rho=1 / 4$, and hence

$$
f^{\prime \prime}(s) \geq 4 \delta^{2} f(s) \quad \text { for }|s| \leq T-1 / 4 .
$$

Now (54) follows from Lemma 5.8 below with $\rho=1 / 4, \delta$ replaced by $2 \delta$, and $T$ replaced by $T-1 / 4$.

Integration of (54) yields

$$
\left\|\partial_{S} A\right\|_{L^{2}([\sigma-3 / 2, \sigma+3 / 2] \times Y)} \leq C^{\prime \prime} e^{-\delta(T-|\sigma|)}\left\|\partial_{S} A\right\|_{L^{2}(([-T, 1-T] \cup[T-1, T]) \times Y)}
$$

for every $\sigma \in[-T+2, T-2]$ with $C^{\prime \prime}=C e^{3 \delta / 2} \delta^{-1 / 2}$. Now, shrinking $\varepsilon$ if necessary, we can apply Lemma 5.7 with $\rho=3 / 2$ and $\rho^{\prime}=1$ to the paths shifted by $\sigma$. Since $\left(\partial_{s} A, 0\right) \in \operatorname{ker} \mathcal{D}_{\mathbb{A}}$ (as in Step 2 of the proof of Theorem 5.1) we obtain constants $C_{k}$ and $C_{k}^{\prime}$ for every $k \geq 0$ such that

$$
\begin{aligned}
\left\|\partial_{S} A\right\|_{\mathcal{C}^{k}([\sigma-1, \sigma+1] \times Y), \mathbb{A}} & \leq C_{k}^{\prime}\left\|\partial_{S} A\right\|_{L^{2}([\sigma-3 / 2, \sigma+3 / 2] \times Y)} \\
& \leq C_{k} e^{-\delta(T-|\sigma|)}\left\|\partial_{S} A\right\|_{L^{2}(([-T, 1-T] \cup[T-1, T]) \times Y)} .
\end{aligned}
$$

for every $\sigma \in[-T+2, T-2]$. Taking the supremum over $\sigma \in[-S+1, s-1]$ then proves the assertion (44) on $\partial_{s} A$.

To prove (45) it remains to estimate the derivatives tangent to $Y$. We fix any two constants $\kappa>0$ and $p>1$ and then, by Lemma 5.5, find a connection $A_{0} \in \mathcal{A}(Y, \mathcal{L})$ such that $F_{A_{0}}+X_{f}\left(A_{0}\right)=0$ and

$$
\left\|A(0)-A_{0}\right\|_{W^{1, p}(Y), A_{0}}+\left\|A(0)-A_{0}\right\|_{L^{\infty}(Y)} \leq \kappa .
$$

After a gauge transformation on $A_{0}$ we can assume that $A(0)$ lies in the local slice $S_{A(0)}$ of $A(0)$, that is $\mathrm{d}_{A_{0}}^{*}\left(A(0)-A_{0}\right)=0$ and $\left.*\left(A(0)-A_{0}\right)\right|_{\partial Y}=0$. Since all critical points are nondegenerate, Corollary 3.6 provides a universal constant $c_{0}$ depending on $q>\max \{3, p\}$ such that for all $\alpha \in \Omega^{1}(Y, \mathfrak{g})$ with $\left.* \alpha\right|_{\partial Y}=0$,

$$
\begin{aligned}
& \|\alpha\|_{L^{\infty}(Y)}+\|\alpha\|_{W^{1, p}(Y), A_{0}} \\
& \quad \leq c_{0}\left(\left\|\mathrm{~d}_{A_{0}} \alpha+\mathrm{d} X_{f}\left(A_{0}\right) \alpha\right\|_{L^{q}(Y)}+\left\|\mathrm{d}_{A_{0}}^{*} \alpha\right\|_{L^{q}(Y)}+\left\|\Pi_{T_{A} \mathcal{L}}^{\perp}\left(\left.\alpha\right|_{\partial Y}\right)\right\|_{L^{q}(\partial Y)}\right) .
\end{aligned}
$$


When applying this to $\alpha=A(0)-A_{0}$ we can use the estimate

$$
\left\|\Pi_{T_{A} \mathcal{L}}^{\perp}\left(\left.\alpha\right|_{\partial Y}\right)\right\|_{L^{q}(\partial Y)} \leq c_{1}\left\|\left.\left(A(0)-A_{0}\right)\right|_{\partial Y}\right\|_{L^{q}(\partial Y)}^{2}
$$

with a uniform constant $c_{1}$ since $\left.A(0)\right|_{\partial Y}$ and $\left.A_{0}\right|_{\partial Y}$ both lie in the submanifold $\mathcal{L} \subset \mathcal{A}^{0, q}(\Sigma)$. More precisely, we abbreviate $A_{0}^{\prime}:=\left.A_{0}\right|_{\partial Y}$, then we can use the exponential map in Lemma E.3 to write

$$
\left.A(0)\right|_{\partial Y}=\Theta_{A_{0}^{\prime}}(\beta)=A_{0}^{\prime}+\beta+\int_{0}^{1}\left(D \Theta_{A_{0}^{\prime}}(\tau \beta)-D \Theta_{A_{0}^{\prime}}(0)\right) \beta \mathrm{d} \tau
$$

for some $\beta \in \mathrm{T}_{A_{0}} \mathcal{L}$, using the identities $\Theta_{A}(0)=A$ and $D \Theta_{A}=\mathrm{Id}$. The map $\Theta$ is smooth and gauge invariant, and $\mathcal{L} / \mathcal{G}(\Sigma)$ is compact, so by the choice of $\kappa>0$ we obtain arbitrarily small bounds on $\|\beta\|_{L^{q}(\Sigma)}$ and a uniform linear bound $\| D \Theta_{A_{0}^{\prime}}(\tau \beta)-$ $D \Theta_{A_{0}^{\prime}}(0)\left\|\leq c^{\prime}\right\| \beta \|_{L^{q}(\Sigma)}$. This implies the uniform estimate

$$
\left\|\left(\left.A(0)\right|_{\partial Y}-A_{0}^{\prime}\right)-\beta\right\|_{L^{q}(\Sigma)} \leq c^{\prime}\|\beta\|_{L^{q}(\Sigma)}^{2} \leq c_{1}\left\|\left.\left(A(0)-A_{0}\right)\right|_{\partial Y}\right\|_{L^{q}(\partial Y)}^{2} .
$$

We also use the identity $\mathrm{d}_{A_{0}} \alpha=F_{A(0)}-F_{A_{0}}-\frac{1}{2}[\alpha \wedge \alpha]$ to obtain

$$
\begin{aligned}
& \left\|A(0)-A_{0}\right\|_{L^{\infty}(Y)}+\left\|A(0)-A_{0}\right\|_{W^{1, p}(Y), A_{0}} \\
& \leq c_{0}\left(\left\|F_{A(0)}+X_{f}(A(0))\right\|_{L^{q}(Y)}+\left\|\frac{1}{2}[\alpha \wedge \alpha]\right\|_{L^{q}(Y)}\right. \\
& \left.\quad+\left\|X_{f}\left(A_{0}+\alpha\right)-X_{f}\left(A_{0}\right)-\mathrm{d} X_{f}\left(A_{0}\right) \alpha\right\|_{L^{q}(Y)}+c_{1}\left\|\left.\alpha\right|_{\partial Y}\right\|_{L^{q}(\partial Y)}^{2}\right) \\
& \leq c_{0}\left\|\partial_{s} A(0)\right\|_{L^{q}(Y)}+c_{2} \kappa\left\|A(0)-A_{0}\right\|_{L^{\infty}(Y)} .
\end{aligned}
$$

Here $c_{2}$ is another uniform constant and we have used Proposition D.1 (v) for the perturbation term. If we choose $\kappa=\left(2 c_{2}\right)^{-1}$ and the corresponding $\varepsilon>0$ from Lemma 5.5 , then this proves

$$
\left\|A(0)-A_{0}\right\|_{L^{\infty}(Y)}+\left\|A(0)-A_{0}\right\|_{W^{1, p}(Y), A_{0}} \leq 2 c_{0}\left\|\partial_{S} A(0)\right\|_{L^{q}(Y)} .
$$

Now (45) follows by integrating over the estimate (44) for $\partial_{s} A$.

Lemma 5.8 For every $\delta>0$ and every $\rho>0$ there exists a constant $C$ such that the following holds. If $T \geq \rho$ and $f:[-T, T] \rightarrow \mathbb{R}$ is a $\mathcal{C}^{2}$-function satisfying

$$
f^{\prime \prime}(s) \geq \delta^{2} f(s), \quad f(s) \geq 0
$$

for all $s \in[-T, T]$, then

$$
f(s) \leq C e^{-\delta(T-|s|)} E_{\rho}(f)
$$


for all $|s| \leq T-\rho$, where

$$
E_{\rho}(f):=\int_{-T}^{\rho-T} f(s) \mathrm{d} s+\int_{T-\rho}^{T} f(s) \mathrm{d} s .
$$

Proof We claim that there is a constant $C_{0}=C_{0}(\delta, \rho)>0$ such that every $\mathcal{C}^{2}$-function $f:[-T, T] \rightarrow \mathbb{R}$ with $T \geq \rho$ that satisfies (55) also satisfies

$$
f^{\prime}(s)-\delta f(s) \geq-C_{0} e^{-\delta T} E_{\rho}(f)
$$

for all $0 \leq s \leq T$. To see this note that, for every $s \in[-T, T]$, we have

$$
\frac{\mathrm{d}}{\mathrm{d} s} e^{\delta s}\left(f^{\prime}(s)-\delta f(s)\right)=e^{\delta s}\left(f^{\prime \prime}(s)-\delta^{2} f(s)\right) \geq 0 .
$$

Hence

$$
f^{\prime}(s)-\delta f(s) \geq e^{\delta(r-s)}\left(f^{\prime}(r)-\delta f(r)\right)
$$

for all $-T \leq r \leq s \leq T$. Integrating this over the interval $t \leq r \leq t+\rho / 2$ for $-T \leq t \leq-\rho / 2-T \leq t \leq \rho / 2-T$ and $s \geq 0$ gives

$$
\begin{aligned}
f^{\prime}(s)-\delta f(s) & \geq \frac{2 e^{-\delta s}}{\rho} \int_{t}^{t+\rho / 2} e^{\delta r}\left(f^{\prime}(r)-\delta f(r)\right) \mathrm{d} r \\
& =\frac{2 e^{-\delta s}}{\rho} \int_{t}^{t+\rho / 2}\left(\frac{\mathrm{d}}{\mathrm{d} r}\left(e^{\delta r} f(r)\right)-2 \delta e^{\delta r} f(r)\right) \mathrm{d} r \\
& \geq-\frac{2}{\rho} e^{\delta t} f(t)-\frac{4 \delta e^{\delta \rho / 2}}{\rho} e^{\delta t} E_{\rho}(f) .
\end{aligned}
$$

Integration over the interval $-T \leq t \leq \rho / 2-T$ yields (57) with $C_{0}:=12 \rho^{-2} e^{\delta \rho}$. By (57), we have

$$
\frac{\mathrm{d}}{\mathrm{d} s} e^{-\delta s} f(s)=e^{-\delta s}\left(f^{\prime}(s)-\delta f(s)\right) \geq-C_{0} e^{-\delta(s+T)} E_{\rho}(f)
$$

for $0 \leq s \leq T$ and hence

$$
e^{-\delta t} f(t)-e^{-\delta s} f(s) \geq-C_{1} e^{-\delta T} E_{\rho}(f)
$$

for $0 \leq s \leq t \leq T$, where $C_{1}:=C_{0} / \delta$. For $s \leq T-\rho \leq t \leq T$ this implies

$$
f(s) \leq e^{\delta(s-t)} f(t)+C_{1} e^{\delta(s-T)} E_{\rho}(f) \leq e^{\delta(s-T)}\left(e^{\delta \rho} f(t)+C_{1} E_{\rho}(f)\right) .
$$

Integrating this inequality over the interval $T-\rho \leq t \leq T$ gives (56) for $0 \leq s \leq T-\rho$ with $C:=C_{1}+\rho^{-1} e^{\delta \rho}$. To prove the estimate for $-T+\rho \leq s \leq 0$ replace $f$ by the function $s \mapsto f(-s)$. 
We close this section with a useful exponential estimate for the solutions of the linearized equation.

Theorem 5.9 Let $A:[0, \infty) \rightarrow \mathcal{A}(Y, \mathcal{L})$ be a finite energy solution of $(42)$ that converges to a nondegenerate critical point $A^{+} \in \mathcal{A}(Y, \mathcal{L})$ of $\mathcal{C} \mathcal{S}_{\mathcal{L}}+h_{f}$. Then there exists a constant $\delta>0$ with the following significance. If $\alpha:[0, \infty) \rightarrow \Omega^{1}(Y, \mathfrak{g})$ is a smooth solution of the equation

$$
\partial_{s} \alpha(s)=*\left(\mathrm{~d}_{A(s)} \alpha+\mathrm{d} X_{f}(A(s)) \alpha(s)\right), \quad \mathrm{d}_{A(s)}^{*} \alpha(s)=0
$$

satisfying the boundary conditions $\left.\alpha(s)\right|_{\Sigma} \in \mathrm{T}_{A(s)} \mathcal{L}$ and $\left.* \alpha(s)\right|_{\Sigma}=0$, and

$$
\int_{0}^{\infty} e^{-\delta s}\|\alpha(s)\|_{L^{2}(Y)}^{2} \mathrm{~d} s<\infty
$$

then there are constants $C_{k}$ such that, for every $s \geq 1$ and every integer $k \geq 0$,

$$
\|\alpha\|_{\mathcal{C}^{k}([s-1, s+1] \times Y)} \leq C_{k} e^{-\delta s} .
$$

Proof We prove first that

$$
\|\alpha(s)\|_{L^{2}(Y)}^{2} \leq C e^{-\delta s} .
$$

Since the limit connection is nondegenerate, Corollary 3.6 provides an estimate

$$
\|\alpha(s)\|_{W^{1,2}(Y)} \leq c\left\|\mathrm{~d}_{A(s)} \alpha+\mathrm{d} X_{f}(A(s)) \alpha(s)\right\|_{L^{2}(Y)}
$$

for $s$ sufficiently large. This implies that the function

$$
g(s):=\frac{1}{2}\|\alpha(s)\|_{L^{2}(Y)}^{2}
$$

satisfies

$$
\begin{aligned}
g^{\prime \prime}(s) & =\left\|\partial_{s} \alpha\right\|_{L^{2}}^{2}+\left\langle\left(\mathrm{d}_{A}+\mathrm{d} X_{f}(A)\right) \partial_{s} \alpha, \alpha\right\rangle+\left\langle\left(\left[\partial_{s} A, \alpha\right]+\mathrm{d}^{2} X_{f}\left(\partial_{s} A, \alpha\right)\right), \alpha\right\rangle \\
& \geq 2\left\|\mathrm{~d}_{A} \alpha+\mathrm{d} X_{f}(A) \alpha\right\|_{L^{2}(Y)}^{2}+\int_{\partial Y}\left\langle\left.\left.\partial_{s} \alpha\right|_{\partial Y} \wedge \alpha\right|_{\partial Y}\right\rangle-C\left\|\partial_{s} A\right\|_{\infty}\|\alpha\|_{L^{2}(Y)}^{2} \\
& \geq 2 c^{-2}\|\alpha\|_{W^{1,2}(Y)}^{2}-C\left\|\partial_{S} A\right\|_{\infty}\left\|\left.\alpha\right|_{\partial Y}\right\|_{L^{2}(\partial Y)}^{2}-C\left\|\partial_{s} A\right\|_{\infty}\|\alpha\|_{L^{2}(Y)}^{2} \\
(59) \quad & \geq \delta^{2} g(s)
\end{aligned}
$$

for some $\delta>0$ and all $s \geq s_{0}$. Here we used Proposition D.1 (v) to estimate $\left\|\mathrm{d}^{2} X_{f}\left(\partial_{s} A, \alpha\right)\right\|_{L^{2}(Y)}$ and Theorem E.1 to write $\left.\alpha(s)\right|_{\partial Y}=P_{\left.A(s)\right|_{\partial Y}} \beta(s)$ for tangent vectors $\beta(s) \in \mathrm{T}_{A_{0}} \mathcal{L}$ at the limit connection $A_{0}:=\left.\lim _{s \rightarrow \infty} A(s)\right|_{\partial Y}$. This gives the estimate

$$
\int_{\partial Y}\left\langle\left.\left.\partial_{s} \alpha\right|_{\partial Y} \wedge \alpha\right|_{\partial Y}\right\rangle=\int_{\partial Y}\left\langle\left.\left(\partial_{S} P_{\left.A(s)\right|_{\partial Y}}\right) \beta \wedge \alpha\right|_{\partial Y}\right\rangle \leq C\left\|\partial_{S} A\right\|_{\infty}\left\|\left.\alpha\right|_{\partial Y}\right\|_{L^{2}(\partial Y)}^{2} .
$$


The final inequality in (59) follows from the exponential decay of $\partial_{s} A$ (see Theorem 5.1) with any $\delta<2 c^{-1}$ and sufficiently large $s_{0}$. This shows that the function $h(s):=$ $e^{-\delta s}\left(g^{\prime}(s)+\delta g(s)\right)$ is monotonically increasing for $s \geq s_{0}$. We claim that $h(s) \leq 0$ for all $s \geq s_{0}$. Suppose otherwise that there is an $s_{1} \geq s_{0}$ such that $c_{1}:=h\left(s_{1}\right)>0$. Then $h(s) \geq c_{1}$ for all $s \geq s_{1}$, hence

$$
\frac{d}{d s}\left(e^{\delta s} g(s)\right)=e^{2 \delta s} h(s) \geq e^{2 \delta s} c_{1}, \quad s \geq s_{1},
$$

and hence, by integration,

$$
e^{\delta s} g(s) \geq \frac{c_{1}}{2 \delta} e^{2 \delta s}-\left(\frac{c_{1}}{2 \delta} e^{2 \delta s_{1}}-e^{\delta s_{1}} g\left(s_{1}\right)\right) .
$$

But this means that the function $s \mapsto e^{-\delta s} g(s)$ is not integrable, in contradiction to our assumption. Thus we have proved that $h(s) \leq 0$ and hence $g^{\prime}(s) \leq-\delta g(s)$ for every $s \geq s_{0}$. Hence either $g$ vanishes identically for all sufficiently large $s$ or $g>0$ for all $s \geq s_{0}$ and $(\log g)^{\prime} \leq-\delta$. This proves (58).

To obtain bounds on the derivatives of $\alpha$ we use Theorem 3.11 (ii) with $\mathcal{D}_{\mathbb{A}}$ replaced by the adjoint $-\mathcal{D}_{\mathbb{A}}^{*}=\nabla_{s}-\mathcal{H}_{A}$. Since $A(s)$ converges in the $C^{\infty}$ topology for $s \rightarrow \infty$ we obtain $\|\alpha\|_{W^{k+1,2}([s-1, s+1] \times Y)} \leq C_{k}\|\alpha\|_{L^{2}([s-2, s+2] \times Y)}$ with a uniform constant $C_{k}$ for each integer $k$ and all $s \geq 2$. The result then follows from the Sobolev embeddings $W^{k+3,2}([-1,1] \times Y) \hookrightarrow \mathcal{C}^{k}([-1,1] \times Y)$.

\section{Moduli spaces and Fredholm theory}

In this section we set up the Fredholm theory for the boundary value problem (16). For the purpose of this paper we could restrict the discussion to the case of a tube $\mathbb{R} \times Y$ as base manifold. In view of a future definition of product structures however, we take some time to introduce a more general class of base manifolds and develop the basic Fredholm theory for these. For the index computations we then restrict to the case of a tube. We begin by introducing the basic setup followed by a discussion of the relevant moduli spaces. The main part of this section then discusses the properties of the linearized operators.

\section{Instanton data}

Definition 6.1 A 4-manifold with boundary space-time splitting and tubular ends is a triple $(X, \tau, \iota)$ consisting of

- an oriented smooth 4-manifold $X$ with boundary, 
- a tuple $\tau=\left(\tau_{1}, \ldots, \tau_{m}\right)$ of orientation preserving embeddings

$$
\tau_{i}: \mathcal{S}_{i} \times \Sigma_{i} \rightarrow \partial X, \quad i=1, \ldots m,
$$

where each $\Sigma_{i}$ is a compact oriented 2-manifold and each $\mathcal{S}_{i}$ is either $\mathbb{R}$ or $S^{1} \cong \mathbb{R} / \mathbb{Z}$,

- a tuple $\iota=\left(\iota_{1}, \ldots, \iota_{n}\right)$ of orientation preserving embeddings

$$
\iota_{j}:(0, \infty) \times Y_{j} \rightarrow X, \quad j=1, \ldots, n,
$$

where $Y_{j}$ is a compact oriented 3-manifold with boundary,

satisfying the following conditions.

(i) The images of the embeddings $\tau_{1}, \ldots, \tau_{m}$ have disjoint closures and

$$
\partial X=\bigcup_{i=1}^{m} \tau_{i}\left(\mathcal{S}_{i} \times \Sigma_{i}\right) .
$$

(ii) For $j=1, \ldots, n$ the image $U_{j}:=\iota_{j}\left((0, \infty) \times Y_{j}\right)$ of $\iota_{j}$ is an open subset of $X$, the closures of the sets $U_{j}$ are pairwise disjoint, and the set $X \backslash \bigcup_{j=1}^{n} U_{j}$ is compact.

(iii) For every $j \in\{1, \ldots, n\}$ there is a subset $I_{j} \subset\{1, \ldots, m\}$ and a map $\varepsilon_{j}: I_{j} \rightarrow$ $\{ \pm 1\}$ such that

$$
\partial Y_{j}=\bigsqcup_{i \in I_{j}} \Sigma_{i}, \quad \iota_{j}(s, z)=\tau_{i}\left(\varepsilon_{j}(i)(s+1), z\right)
$$

for $s>0, i \in I_{j}$, and $z \in \Sigma_{i}$. The orientation of $\Sigma_{i}$ coincides with the boundary orientation of $Y_{j}$ iff $\varepsilon_{j}(i)=-1$.

Definition 6.2 Let $(X, \tau, \iota)$ be a 4-manifold with boundary space-time splitting and tubular ends. A Riemannian metric $g$ on $X$ is called compatible with the boundary space-time splitting and the tubular ends if

(i) on each tubular end the metric is of split form

$$
\iota_{j}^{*} g=\mathrm{d} s^{2}+g_{j},
$$

where $g_{j}$ is a metric on $Y_{j}$ independent of $s \in(0, \infty)$,

(ii) each $\tau_{i}$ can be extended to an embedding $\bar{\tau}_{i}: \mathcal{S}_{i} \times\left[0, \varepsilon_{i}\right) \times \Sigma_{i} \rightarrow X$ for some $\varepsilon_{i}>0$ such that

$$
\bar{\tau}_{i}^{*} g=\mathrm{d} s^{2}+\mathrm{d} t^{2}+g_{i, s, t},
$$

where $g_{i, s, t}$ is a smooth family of metrics on $\Sigma_{i}$. 
A quadruple $(X, \tau, \iota, g)$ with these properties is called a Riemannian 4-manifold with boundary space-time splitting and tubular ends.

Remark 6.3 (i) On the tubular ends condition (ii) in Definition 6.2 follows from (i). Indeed, on $U_{j}$ the extension $\bar{\tau}_{i}$ for $i \in I_{j}$ is obtained by composing $\iota_{j}$ with the embedding $[0, \varepsilon) \times \Sigma_{i} \rightarrow Y_{j}$ associated to geodesic normal coordinates.

(ii) Let $(X, \tau, \iota, g)$ be a Riemannian 4-manifold with boundary space-time splitting and tubular ends. Then $X$ can be exhausted by compact deformation retracts. Hence the triple $(X, \tau, g)$ is a Riemannian 4-manifold with a boundary space-time splitting in the sense of [35, Definition 1.2].

Example 6.4 Let $Y$ be a compact oriented 3-manifold with nonempty boundary $\partial Y=\Sigma$. Then $X:=\mathbb{R} \times Y$ satisfies the requirements of Definition 6.1 with the obvious inclusion $\tau: \mathbb{R} \times \Sigma \rightarrow \partial X, Y_{1}:=Y, Y_{2}:=\bar{Y}$ (which has the reversed orientation), $\iota_{1}(s, y):=(s+1, y), \iota_{2}(s, y):=(-s-1, y)$. For any metric $g_{Y}$ on $Y$ the metric $\mathrm{d} s^{2}+g_{Y}$ on $\mathbb{R} \times Y$ satisfies the conditions of Definition 6.2. If $g_{ \pm}$are two metrics on $Y$ then, by [35, Example 1.4], there is a metric $g$ on $\mathbb{R} \times Y$ that satisfies the conditions of Definition 6.2 and has the form $g=\mathrm{d} s^{2}+g_{ \pm}$for $\pm s \geq 1$.

The following result will be needed in the proof of independence of the Floer homology from the choice of a metric.

Lemma 6.5 Let $(X, \tau, \iota)$ be a 4-manifold with boundary space-time splitting and tubular ends and, for $j=1, \ldots, n$, let $g_{j}$ be a metric on $Y_{j}$. Then there is a metric $g$ on $X$, compatible with the boundary space-time splitting and the tubular ends, such that (i) in Definition 6.2 holds with the given metrics $g_{j}$.

Moreover, the space of such metrics $g$ is contractible if we restrict the consideration to those metrics with $\varepsilon_{i} \geq \varepsilon$ in (ii) for any fixed $\varepsilon>0$.

Proof The construction of a metric with given ends works as in [35, Example 1.4]. Denote by $\operatorname{Met}(X, \tau, \iota)$ the set of metrics on $X$ that satisfy (i) in Definition 6.2 and $\tau_{i}^{*} g=\mathrm{d} s^{2}+g_{i, s}$ for $i=1, \ldots, m$ and some families of metrics $\left(g_{i, s}\right)_{s \in \mathcal{S}_{i}}$ on $\Sigma_{i}$. Then $\operatorname{Met}(X, \tau, \iota)$ is convex and hence contractible. Fix $\varepsilon>0$ and let $\operatorname{Met}_{\varepsilon}(X, \tau, \iota) \subset$ $\operatorname{Met}(X, \tau, \iota)$ denote the subset of all metrics that are compatible with the boundary space-time splitting and the tubular ends as in Definition 6.2 with $\varepsilon_{i} \geq \varepsilon$ in (ii). To prove that $\operatorname{Met}_{\varepsilon}(X, \tau, \iota)$ is contractible it suffices to construct a continuous left inverse of the inclusion $\operatorname{Met}_{\varepsilon}(X, \tau, \iota) \hookrightarrow \operatorname{Met}(X, \tau, \iota)$. 
Every metric $g \in \operatorname{Met}(X, \tau, \iota)$ determines embeddings

defined by

$$
\bar{\tau}_{g, i}: \mathcal{S}_{i} \times[0, \delta) \times \Sigma_{i} \rightarrow X
$$

$$
\bar{\tau}_{g, i}(s, t, z):=\exp _{\tau_{i}(s, z)}\left(t v_{i}(s, z)\right)
$$

where $v_{i}: \mathcal{S}_{i} \times \Sigma_{i} \rightarrow \tau_{i}^{*} \mathrm{~T} X$ denotes the inward unit normal. The constant $\delta>0$ for which the $\bar{\tau}_{g, i}$ are embeddings can be chosen uniform on a $\mathcal{C}^{1}$-neighbourhood of the metric. Taking a locally finite refinement of the cover of $\operatorname{Met}(X, \tau, \iota)$ by these neighbourhoods and using a partition of unity one can construct a function

$$
\bar{\delta}: \operatorname{Met}(X, \tau, \iota) \rightarrow(0, \varepsilon],
$$

continuous with respect to the $\mathcal{C}^{\infty}$-topology, such that the maps $\bar{\tau}_{g, i}$ are embeddings for $0<\delta \leq \bar{\delta}(g)$.

For $g \in \operatorname{Met}(X, \tau, \iota)$ and $i=1, \ldots, m$ let us define the metrics $h_{g, i}$ on the strips $\mathcal{S}_{i} \times[0, \bar{\delta}(g)) \times \Sigma_{i}$ by

$$
h_{g, i}:=\mathrm{d} s^{2}+\mathrm{d} t^{2}+g_{i, s, t},
$$

where the metric $g_{i, s, t}$ on $\Sigma_{i}$ is the pullback of the metric on $X$ under the embedding $z \mapsto \bar{\tau}_{g, i}(s, t, z)$. We fix a smooth cutoff function $\lambda:[0,1] \rightarrow[0,1]$ such that $\lambda(t)=0$ for $t$ near 0 and $\lambda(t)=1$ for $t$ near 1 . Then for $\delta>0$ we define $\lambda_{\delta}: \mathcal{S}_{i} \times[0, \delta) \times \Sigma_{i} \rightarrow[0,1]$ by

$$
\lambda_{\delta}(s, t, z):=\lambda(t / \delta)
$$

Now we can define the map $\operatorname{Met}(X, \iota, \tau) \rightarrow \operatorname{Met}_{\varepsilon}(X, \iota, \tau): g \mapsto \tilde{g}$ by

$$
\widetilde{g}:=\left(\bar{\tau}_{g, i}\right)_{*}\left(\lambda_{\bar{\delta}(g)} \bar{\tau}_{g, i}^{*} g+(1-\lambda \bar{\delta}(g)) h_{g, i}\right)
$$

on the image of $\bar{\tau}_{g, i}$ for $i=1, \ldots, m$ and by $\tilde{g}:=g$ on the complement. This map is the identity on $\operatorname{Met}_{\varepsilon}(X, \tau, \iota)$ since $\varepsilon_{i} \geq \varepsilon \geq \bar{\delta}(g)$. So we have constructed the required left inverse of the inclusion $\operatorname{Met}_{\varepsilon}(X, \tau, \iota) \hookrightarrow \operatorname{Met}(X, \tau, \iota)$.

Definition 6.6 Let $(X, \tau, \iota)$ be a 4-manifold with boundary space-time splitting and tubular ends. Instanton data on $X$ are given by a triple $(g, \mathcal{L}, f)$ with the following properties.

- $g$ is a Riemannian metric on $X$ compatible with the boundary space-time splitting and the tubular ends.

- $\mathcal{L}=\left(\mathcal{L}_{1}, \ldots, \mathcal{L}_{m}\right)$ is an $m$-tuple of gauge invariant, monotone Lagrangian submanifolds $\mathcal{L}_{i} \subset \mathcal{A}\left(\Sigma_{i}\right)$, satisfying (L1)-(L2). 
- $X_{f}: \mathcal{A}(X) \rightarrow \Omega^{2}(X, \mathfrak{g})$ is a holonomy perturbation as in the introduction such that, on every tubular end and for every $\mathbb{A} \in \mathcal{A}(X)$, the 2 -form $\iota_{j}^{*} X_{f}(\mathbb{A}) \in$ $\Omega^{2}\left((0, \infty) \times Y_{j}, \mathfrak{g}\right)$ is induced by the path $s \mapsto X_{f_{j}}\left(A_{j}(s)\right)$, where $\iota_{j}^{*} \mathbb{A}=$ : $A_{j}(s)+\Phi_{j}(s) \mathrm{d} s$. Here $X_{f_{j}}: \mathcal{A}\left(Y_{j}\right) \rightarrow \Omega^{2}\left(Y_{j}, \mathfrak{g}\right)$ is as in (9). The perturbation $f$ involves a choice of thickened loops, ie embeddings $\gamma_{i}: S^{1} \times \Omega \rightarrow \operatorname{int}(X)$, where $\Omega \subset \mathbb{R}^{3}$ is a contractible open set.

\section{The moduli space}

Let $(X, \tau, \iota)$ be a 4 -manifold with boundary space-time splitting and tubular ends and let $(g, \mathcal{L}, f)$ be instanton data on $X$. The perturbed anti-self-duality equation with Lagrangian boundary conditions has the form

$$
F_{\mathbb{A}}+X_{f}(\mathbb{A})+*\left(F_{\mathbb{A}}+X_{f}(\mathbb{A})\right)=0, \quad \tau_{i, s}^{*} \mathbb{A} \in \mathcal{L}_{i} \quad \forall s \in \mathcal{S}_{i} .
$$

Here the embedding $\tau_{i, s}: \Sigma_{i} \rightarrow X$ is defined by $\tau_{i, s}(z):=\tau_{i}(s, z)$. The energy of a solution is

$$
E_{f}(\mathbb{A}):=\frac{1}{2} \int_{X}\left|F_{\mathbb{A}}+X_{f}(\mathbb{A})\right|^{2} .
$$

By Theorem 5.1 every finite energy solution of (60) that is in temporal gauge on the tubular ends converges to critical points $A_{j}$ of the perturbed Chern-Simons functionals, ie

$$
\lim _{s \rightarrow \infty}\left\|\iota_{j}^{*} \mathbb{A}-A_{j}\right\|_{\mathcal{C}^{k}\left([s-1, s+1] \times Y_{j}\right)}=0
$$

for every $j \in\{1, \ldots, n\}$ and every integer $k \geq 0$. This equation is understood as follows. We denote by $\mathcal{A}(X, \mathcal{L})$ the set of smooth connections $\mathbb{A} \in \mathcal{A}(X)$ that satisfy the Lagrangian boundary conditions $\tau_{i, s}^{*} \mathbb{A} \in \mathcal{L}_{i}$ for all $i \in\{1, \ldots, m\}$ and $s \in \mathcal{S}_{i}$. On a tubular end, any such connection decomposes as

$$
\iota_{j}^{*} \mathbb{A}=B_{j}+\Phi_{j} \mathrm{~d} s
$$

with $\Phi_{j}:(0, \infty) \rightarrow \Omega^{0}\left(Y_{j}, \mathfrak{g}\right)$ and $B_{j}:(0, \infty) \rightarrow \mathcal{A}\left(Y_{j}, \mathcal{L}\right)$. Here $\mathcal{A}\left(Y_{j}, \mathcal{L}\right)$ denotes the set of smooth connections $B \in \mathcal{A}\left(Y_{j}\right)$ that satisfy the Lagrangian boundary conditions $\left.B\right|_{\Sigma_{i}} \in \mathcal{L}_{i}$ for all $i \in I_{j}$. The temporal gauge condition means that $\Phi_{j} \equiv 0$. For $j=1, \ldots, n$ the connection $A_{j} \in \mathcal{A}\left(Y_{j}, \mathcal{L}\right)$ in (61) is a critical point of the perturbed Chern-Simons functional for $Y_{j}$, ie

$$
F_{A_{j}}+X_{f_{j}}\left(A_{j}\right)=0 .
$$

The space of solutions of (60) and (61) that are in temporal gauge on the tubular ends will be denoted by

$$
\widetilde{\mathcal{M}}\left(A_{1}, \ldots, A_{n} ; X_{f}\right) \subset \mathcal{A}(X, \mathcal{L}) .
$$


Let us denote by $\mathcal{G}_{A_{j}} \subset \mathcal{G}\left(Y_{j}\right)$ the isotropy subgroup of $A_{j}$. So the group $\mathcal{G}\left(A_{1}, \ldots, A_{n}\right)$ of all gauge transformations $u \in \mathcal{G}(X)$ that satisfy $u \circ \iota_{j} \equiv u_{j} \in \mathcal{G}_{A_{j}}$ for $j=1, \ldots, n$, acts on the space $\widetilde{\mathcal{M}}\left(A_{1}, \ldots, A_{n} ; X_{f}\right)$. The quotient will be denoted by

$$
\mathcal{M}\left(A_{1}, \ldots, A_{n} ; X_{f}\right):=\widetilde{\mathcal{M}}\left(A_{1}, \ldots, A_{n} ; X_{f}\right) / \mathcal{G}\left(A_{1}, \ldots, A_{n}\right) .
$$

In the case of the tube $X=\mathbb{R} \times Y$, this moduli space can easily be identified with the one that is mentioned in the introduction. Similarly, the moduli space $\mathcal{M}\left(A_{1}^{\prime}, \ldots, A_{n}^{\prime} ; X_{f}\right)$ for gauge equivalent limits $A_{i}^{\prime} \in\left[A_{i}\right]$ can be identified with $\mathcal{M}\left(A_{1}, \ldots, A_{n} ; X_{f}\right)$.

\section{The linearized operator}

Fix critical points $A_{j} \in \mathcal{A}\left(Y_{j}, \mathcal{L}\right), j=1, \ldots, n$, of the perturbed Chern-Simons functionals and let $\mathbb{A} \in \mathcal{A}(X, \mathcal{L})$ be a connection satisfying (61). Denote by $\Omega_{\mathbb{A}}^{1}(X, \mathfrak{g})$ the space of smooth $1-$ forms that satisfy the boundary conditions

$$
\left.* \alpha\right|_{\partial X}=0, \quad \tau_{i, s}^{*} \alpha \in \mathrm{T}_{\tau_{i, s}^{*} \mathbb{A}} \mathcal{L}_{i}
$$

for $i \in\{1, \ldots, m\}$ and $s \in \mathcal{S}_{i}$. Then $\mathbb{A}$ determines a differential operator

$$
\begin{aligned}
\mathcal{D}_{\mathbb{A}} & : \Omega_{\mathbb{A}}^{1}(X, \mathfrak{g}) \rightarrow \Omega^{2,+}(X, \mathfrak{g}) \times \Omega^{0}(X, \mathfrak{g}), \\
\mathcal{D}_{\mathbb{A}} \alpha & :=\left(\left(\mathrm{d}_{\mathbb{A}} \alpha+\mathrm{d} X_{f}(\mathbb{A}) \alpha\right)^{+},-\mathrm{d}_{\mathbb{A}}^{*} \alpha\right),
\end{aligned}
$$

where $\omega^{+}:=\frac{1}{2}(\omega+* \omega)$ denotes the self-dual part of a 2 -form $\omega \in \Omega^{2}(X, \mathfrak{g})$. This is a generalization of the linearized operator on $\mathbb{R} \times Y$ in (31). The formal adjoint operator

$$
\mathcal{D}_{\mathbb{A}}^{*}: \Omega_{\mathbb{A}}^{2,+}(X, \mathfrak{g}) \times \Omega^{0}(X, \mathfrak{g}) \rightarrow \Omega^{1}(X, \mathfrak{g})
$$

is given by $\quad \mathcal{D}_{\mathbb{A}}^{*}(\omega, \varphi)=\mathrm{d}_{\mathbb{A}}^{*} \omega+\mathrm{d} X_{f}(\mathbb{A})^{*} \omega-\mathrm{d}_{\mathbb{A}} \varphi$.

Here $\Omega_{\mathbb{A}}^{2,+}(X, \mathfrak{g})$ denotes the space of self-dual 2-forms $\omega$ on $X$ that satisfy the boundary condition

$$
\tau_{i, s}^{*} \omega=0,\left.\quad \iota(\partial / \partial s) \tau_{i}^{*} \omega\right|_{\{s\} \times \Sigma} \in \mathrm{T}_{\tau_{i, s}^{*} \mathbb{A}} \mathcal{L}_{i}
$$

for $i \in\{1, \ldots, m\}$ and $s \in \mathcal{S}_{i}$.

To obtain a Fredholm operator we must impose decay conditions on $\alpha$ at the tubular ends and extend the operator to suitable Sobolev completions. For any integer $k \geq 1$ and any $p>1$ denote by $W_{\mathbb{A}}^{k, p}\left(X, \mathrm{~T}^{*} X \otimes \mathfrak{g}\right)$ the space of 1 -forms on $X$ of class $W^{k, p}$ with values in $\mathfrak{g}$ that satisfy the boundary conditions $(63)^{4}$ and by $W_{\mathbb{A}}^{k, p}\left(X, \Lambda^{2,+} \mathrm{T}^{*} X \otimes \mathfrak{g}\right)$

\footnotetext{
${ }^{4}$ Note that the subscript $\mathbb{A}$ in $W_{\mathbb{A}}^{k, p}$ indicates boundary conditions for the 1 -forms in this space. This is not to be confused with the norms $\|\cdot\|_{W^{k, p}, \mathbb{A}}$ in Remark 5.2, where the subscript indicates that the covariant derivatives are twisted by $\mathbb{A}$.
} 
the space of self-dual 2-forms on $X$ of class $W^{k, p}$ with values in $\mathfrak{g}$ that satisfy the boundary conditions (65). The following theorem summarizes the Fredholm properties of $\mathcal{D}_{\mathbb{A}}$ and $\mathcal{D}_{\mathbb{A}}^{*}$. The regularity results (ii) and (iii) are steps towards the proof of (i).

Theorem 6.7 Suppose the limit connections $A_{j}$ are nondegenerate and irreducible, ie $H_{A_{j}}^{0}=0$ and $H_{A_{j}}^{1}, f_{j}=0$ for $j=1, \ldots, n$. Then the following holds for every connection $\mathbb{A} \in \mathcal{A}(X, \mathcal{L})$ that satisfies (61).

(i) The operators

$$
\begin{aligned}
& \mathcal{D}_{\mathbb{A}}: W_{\mathbb{A}}^{k, p}\left(X, \mathrm{~T}^{*} X \otimes \mathfrak{g}\right) \rightarrow W^{k-1, p}\left(X, \Lambda^{2,+} \mathrm{T}^{*} X \otimes \mathfrak{g}\right) \times W^{k-1, p}(X, \mathfrak{g}), \\
& \mathcal{D}_{\mathbb{A}}^{*}: W_{\mathbb{A}}^{k, p}\left(X, \Lambda^{2,+} \mathrm{T}^{*} X \otimes \mathfrak{g}\right) \times W^{k, p}(X, \mathfrak{g}) \rightarrow W^{k-1, p}\left(X, \mathrm{~T}^{*} X \otimes \mathfrak{g}\right)
\end{aligned}
$$

are Fredholm for every integer $k \geq 1$ and every $p>1$. Their Fredholm indices

$$
\delta_{f}(\mathbb{A}):=\operatorname{index} \mathcal{D}_{\mathbb{A}}=-\operatorname{index} \mathcal{D}_{\mathbb{A}}^{*}
$$

are independent of $k$ and $p$ and depend only on the homotopy class of $\mathbb{A}$ subject to $(61)$.

(ii) If $\alpha \in L^{p}\left(X, \mathrm{~T}^{*} X \otimes \mathfrak{g}\right), \omega \in W^{k-1, p}\left(X, \Lambda^{2,+} \mathrm{T}^{*} X \otimes \mathfrak{g}\right), \varphi \in W^{k-1, p}(X, \mathfrak{g})$ satisfy the equation

$$
\int_{X}\left\langle\mathcal{D}_{\mathbb{A}}^{*}\left(\omega^{\prime}, \varphi^{\prime}\right), \alpha\right\rangle=\int_{X}\left(\left\langle\omega^{\prime}, \omega\right\rangle+\left\langle\varphi^{\prime}, \varphi\right\rangle\right)
$$

for every compactly supported smooth $\left(\omega^{\prime}, \varphi^{\prime}\right) \in \Omega_{\mathbb{A}}^{2,+}(X, \mathfrak{g}) \times \Omega^{0}(X, \mathfrak{g})$, then

$$
\alpha \in W_{\mathbb{A}}^{k, p}\left(X, \mathrm{~T}^{*} X \otimes \mathfrak{g}\right) \quad \text { and } \quad \mathcal{D}_{\mathbb{A}} \alpha=(\omega, \varphi) .
$$

(iii) If $\omega \in L^{p}\left(X, \Lambda^{2,+} \mathrm{T}^{*} X \otimes \mathfrak{g}\right), \varphi \in L^{p}(X, \mathfrak{g}), \alpha \in W^{k-1, p}\left(X, \mathrm{~T}^{*} X \otimes \mathfrak{g}\right)$ satisfy the equation

$$
\int_{X}\left\langle(\omega, \varphi), \mathcal{D}_{\mathbb{A}} \alpha^{\prime}\right\rangle=\int_{X}\left\langle\alpha, \alpha^{\prime}\right\rangle
$$

for every compactly supported smooth 1 -form $\alpha^{\prime} \in \Omega_{\mathbb{A}}^{1}(X, \mathfrak{g})$, then we have

$$
\omega \in W_{\mathbb{A}}^{k, p}\left(X, \Lambda^{2,+} \mathrm{T}^{*} X \otimes \mathfrak{g}\right), \quad \varphi \in W^{k, p}(X, \mathfrak{g}) \quad \text { and } \quad \mathcal{D}_{\mathbb{A}}^{*}(\omega, \varphi)=\alpha .
$$

Proof Assertions (ii) and (iii) follow from Theorem 3.11 and Remark 3.10. (To obtain global $W^{k, p}$-regularity one sums up estimates on compact domains - with and without boundary - exhausting $X$.) To prove (i) we combine Theorems 3.11 and 3.13 with a cutoff function argument to obtain the estimate

$$
\|\alpha\|_{W^{k, p}(X)} \leq c\left(\left\|\mathcal{D}_{\mathbb{A}} \alpha\right\|_{W^{k-1, p}(X)}+\|\alpha\|_{W^{k-1, p}(K)}\right)
$$


for a sufficiently large compact subset $K \subset X$. (See Donaldson [10, p 50] or Robbin and Salamon [26] for the case $X=\mathbb{R} \times Y, k=0$, and $p=2$ ). This estimate shows that $\mathcal{D}_{\mathbb{A}}$ has a finite dimensional kernel and a closed image. (See for example [21, Lemma A.1.1].) By (iii) the cokernel of $\mathcal{D}_{\mathbb{A}}$ agrees with the kernel of $\mathcal{D}_{\mathbb{A}}^{*}$. Since $\mathcal{D}_{\mathbb{A}}^{*}$ satisfies a similar estimate as (68), it follows that the cokernel is finite dimensional as well. Hence $\mathcal{D}_{\mathbb{A}}$ and $\mathcal{D}_{\mathbb{A}}^{*}$ are Fredholm operators. By (ii) and (iii), their Fredholm indices add up to zero and are independent of $k$ and $p$. That they depend only on the homotopy class of $\mathbb{A}$ follows from the stability properties of the Fredholm index.

In the case $\partial X=\varnothing$ the space of connections satisfying (61) is convex and so the index of $\mathcal{D}_{\mathbb{A}}$ depends only on the limit connections $A_{j}$. The change of the index under gauge transformations on $Y_{j}$ depends on the degrees of the gauge transformations. By contrast, in the case $\partial X \neq \varnothing$ and $\partial Y_{j} \neq \varnothing$ the space of gauge transformations on $Y_{j}$ is connected, but the Lagrangian submanifolds $\mathcal{L}_{i}$ have nontrivial fundamental groups. So the index of $\mathcal{D}_{\mathbb{A}}$ also depends on the homotopy classes of the paths in $\mathcal{L}_{i}$ that are given by $\left.\mathbb{A}\right|_{\partial X}$.

\section{Weighted theory}

In order to deal with reducible critical points we set up a refined Fredholm theory on weighted Sobolev spaces. Fix small nonzero real numbers $\delta_{1}, \ldots, \delta_{n}$ and choose a smooth function $w: X \rightarrow(0, \infty)$ such that on all tubular ends

$$
w\left(\iota_{j}(s, y)\right)=e^{\delta_{j} s} \quad \text { for } s \geq 1,
$$

$w$ is independent of $y \in Y_{j}$ for $s \in[0,1]$, and $w \equiv 1$ on the complement. We introduce the weighted spaces

$$
W_{\mathbb{A}, \delta}^{k, p}\left(X, \mathrm{~T}^{*} X \otimes \mathfrak{g}\right):=\left\{\alpha: X \rightarrow \mathrm{T}^{*} X \otimes \mathfrak{g} \mid w \alpha \in W_{\mathbb{A}}^{k, p}\left(X, \mathrm{~T}^{*} X \otimes \mathfrak{g}\right)\right\},
$$

and similarly for $W_{\delta}^{k, p}(X, \mathfrak{g})$ and $W_{\delta}^{k, p}\left(X, \Lambda^{2,+} \mathrm{T}^{*} X \otimes \mathfrak{g}\right)$. The function $w$ does not appear in the notation because the spaces only depend on the choice of the $\delta_{j}$. The weighted inner product on $L_{\delta}^{2}\left(X, \mathrm{~T}^{*} X \otimes \mathfrak{g}\right)$ is

$$
\langle\alpha, \beta\rangle_{L_{\delta}^{2}}:=\int_{X} w^{2}\langle\alpha \wedge * \beta\rangle,
$$

and similarly for $L_{\delta}^{2}(X, \mathfrak{g})$. The adjoint operator of $\mathrm{d}_{\mathbb{A}}$ with respect to these two inner products is given by

$$
\mathrm{d}_{\mathbb{A}}^{*, \delta}:=w^{-2} \mathrm{~d}_{\mathbb{A}}^{*} w^{2}: W_{\mathbb{A}, \delta}^{k, p}\left(X, \mathrm{~T}^{*} X \otimes \mathfrak{g}\right) \rightarrow W_{\delta}^{k-1, p}(X, \mathfrak{g}) .
$$

It has the form $(\alpha, \varphi) \mapsto \mathrm{d}_{A}^{*} \alpha-\nabla_{s} \varphi-2 \delta_{j} \varphi$ on the tubular ends. We will be using the following generalized Hodge decomposition. 
Lemma 6.8 Let $k$ be a positive integer and $p>1$ and suppose $\mathbb{A} \in \mathcal{A}(X, \mathcal{L})$ satisfies (61). Then the operator

$$
\mathrm{d}_{\mathbb{A}}^{*, \delta} \mathrm{d}_{\mathbb{A}}: W_{\mathbb{A}, \delta}^{k+1, p}(X, \mathfrak{g}) \rightarrow W_{\delta}^{k-1, p}(X, \mathfrak{g})
$$

with domain $W_{\mathbb{A}, \delta}^{k+1, p}(X, \mathfrak{g}):=\left\{\xi \in W_{\delta}^{k+1, p}(X, \mathfrak{g})\left|* \mathrm{~d}_{\mathbb{A}} \xi\right|_{\partial X}=0\right\}$ is bijective and there is a Hodge decomposition

$$
W_{\mathbb{A}, \delta}^{k, p}\left(X, \mathrm{~T}^{*} X \otimes \mathfrak{g}\right)=\operatorname{kerd}_{\mathbb{A}}^{*, \delta} \oplus \mathrm{d}_{\mathbb{A}} W_{\mathbb{A}, \delta}^{k+1, p}(X, \mathfrak{g}),
$$

Proof This Hodge decomposition is standard (see eg [10, Section 4.3]) except for the boundary conditions. The two subspaces do not intersect since

$$
\left\langle\mathrm{d}_{\mathbb{A}} \xi, \alpha\right\rangle_{L_{\delta}^{2}}-\left\langle\xi, \mathrm{d}_{\mathbb{A}}^{*, \delta} \alpha\right\rangle_{L_{\delta}^{2}}=\int_{\partial X} w^{2}\langle\xi, * \alpha\rangle=0
$$

for all $\alpha \in W_{\mathbb{A}, \delta}^{k, p}\left(X, \mathrm{~T}^{*} X \otimes \mathfrak{g}\right)$. Assuming the operator $\mathrm{d}_{\mathbb{A}}^{*, \delta} \mathrm{d}_{\mathbb{A}}$ is bijective we obtain the Hodge decomposition of $\beta \in W_{\mathbb{A}, \delta}^{k, p}\left(X, \mathrm{~T}^{*} X \otimes \mathfrak{g}\right)$ by solving the Neumann problem

$$
\mathrm{d}_{\mathbb{A}}^{*, \delta} \mathrm{d}_{\mathbb{A}} \xi=\mathrm{d}_{\mathbb{A}}^{*, \delta} \beta,\left.\quad * \mathrm{~d}_{\mathbb{A}} \xi\right|_{\partial X}=0
$$

for $\xi \in W_{\delta}^{k+1, p}(X, \mathfrak{g})$. Since $\mathrm{d}_{\mathbb{A}} \xi$ satisfies the Lagrangian boundary condition we have $\alpha:=\beta-\mathrm{d}_{\mathbb{A}} \xi \in W_{\mathbb{A}, \delta}^{k, p}\left(X, \mathrm{~T}^{*} X \otimes \mathfrak{g}\right)$.

To prove that the operator $\mathrm{d}_{\mathbb{A}}^{*, \delta} \mathrm{d}_{\mathbb{A}}$ is bijective we work with the weight function $w=e^{V}: X \rightarrow(0, \infty)$ given by $V(s)=\delta_{j} s$ on the tubular ends. Since $w$ has normal derivative zero the function $\xi^{\prime}:=w \xi \in W^{k+1, p}(X, \mathfrak{g})$ satisfies the boundary condition $\left.* \mathrm{~d}_{\mathbb{A}} \xi^{\prime}\right|_{\partial X}=0$ whenever $\xi$ does. On the tubular ends we have

$$
w \mathrm{~d}_{\mathbb{A}}^{*, \delta} \mathrm{d}_{\mathbb{A}} w^{-1}=\mathrm{d}_{A_{j}}^{*} \mathrm{~d}_{A_{j}}-\nabla_{s} \nabla_{s}+\delta_{j}^{2} .
$$

This operator is bijective on $W_{\mathbb{A}}^{k+1, p}\left(\mathbb{R} \times Y_{j}, \mathfrak{g}\right)$ since it is Fredholm, symmetric, and positive definite. So, as in the proof of Theorem 6.7, one can use a cutoff function argument to show that $\mathrm{d}_{\mathbb{A}}^{*, \delta} \mathrm{d}_{\mathbb{A}}$ is a Fredholm operator. Partial integration then shows that its kernel and cokernel are equal to the kernel of $\mathrm{d}_{\mathbb{A}}$. To prove that the kernel is zero let $\xi \in W_{\mathbb{A}, \delta}^{k+1, p}(X, \mathfrak{g})$ with $\mathrm{d}_{\mathbb{A}} \xi=0$ and assume w.l.o.g. that $\mathbb{A}$ is in temporal gauge on the tubular ends. Then on each tubular end we have $\partial_{s} \xi_{j} \equiv 0$, hence $\xi_{j} \equiv 0$ by the decay condition, and hence $\xi \equiv 0$. This proves the lemma.

Every connection $\mathbb{A} \in \mathcal{A}(X, \mathcal{L})$ that satisfies (61) determines a differential operator

$$
\mathcal{D}_{\mathbb{A}, \delta}: W_{\mathbb{A}, \delta}^{k, p}\left(X, \mathrm{~T}^{*} X \otimes \mathfrak{g}\right) \rightarrow W_{\delta}^{k-1, p}\left(X, \Lambda^{2,+} \mathrm{T}^{*} X \otimes \mathfrak{g}\right) \times W_{\delta}^{k-1, p}(X, \mathfrak{g})
$$

given by

$$
\mathcal{D}_{\mathbb{A}, \delta} \alpha:=\left(\left(\mathrm{d}_{\mathbb{A}} \alpha+\mathrm{d} X_{f}(\mathbb{A}) \alpha\right)^{+},-\mathrm{d}_{\mathbb{A}}^{*, \delta} \alpha\right) .
$$

Different choices of $w$ with the same $\delta_{j}$ give rise to compact perturbations of $\mathcal{D}_{\mathbb{A}, \delta}$. 
Theorem 6.9 For $j=1, \ldots, n$ let $A_{j} \in \mathcal{A}\left(Y_{j}, \mathcal{L}\right)$ and $\mathbb{A} \in \mathcal{A}(X, \mathcal{L})$ such that $F_{A_{j}}+X_{f_{j}}\left(A_{j}\right)=0$ and $\mathbb{A} \in \mathcal{A}(X, \mathcal{L})$ satisfies (61). Then the following holds.

(i) The operator $\mathcal{D}_{\mathbb{A}, \delta}$ is Fredholm for every integer $k \geq 1$, every $p>1$, and every $n$-tuple of sufficiently small nonzero real numbers $\delta_{1}, \ldots, \delta_{n}$.

(ii) The Fredholm index of $\mathcal{D}_{\mathbb{A}, \delta}$ is independent of $k$ and $p$; it depends only on the signs of the $\delta_{j}$ and on the homotopy class of $\mathbb{A}$ subject to (61).

(iii) If the limit connections $A_{j}$ are all nondegenerate and irreducible, then we have index $\mathcal{D}_{\mathbb{A}, \delta}=\operatorname{index} \mathcal{D}_{\mathbb{A}}$.

(iv) If the limit connections $A_{j}$ are all nondegenerate and $\mathbb{A}$ satisfies (60) then the cokernel of $\mathcal{D}_{\mathbb{A}, \delta}$ is independent of the weight function (up to natural isomorphisms) as long as the $\left|\delta_{j}\right|$ are sufficiently small.

Proof The operator $w \mathcal{D}_{\mathbb{A}, \delta} w^{-1}$ differs from $\mathcal{D}_{\mathbb{A}}$ by a zeroth order perturbation which makes the operators on the tubular ends invertible. Hence assertions (i-iii) follow by adapting the proof of Theorem 6.7 to the present case. To prove (iv) we observe that the restriction of the second component $\mathrm{d}_{\mathbb{A}}^{*, \delta}$ of $\mathcal{D}_{\mathbb{A}, \delta}$ to the image of $\mathrm{d}_{\mathbb{A}}$ is surjective and, when $\mathbb{A}$ satisfies (60), the image of $d_{\mathbb{A}}$ is contained in the kernel of the first component $\left(\mathrm{d}_{\mathbb{A}}+\mathrm{d} X_{f}(\mathbb{A})\right)^{+}$of $\mathcal{D}_{\mathbb{A}, \delta}$. Hence every element in the cokernel of $\mathcal{D}_{\mathbb{A}, \delta}$ has the form $(\eta, 0)$. Moreover, $(\eta, 0)$ belongs to the kernel of the adjoint operator $\mathcal{D}_{\mathbb{A}, \delta}^{*, \delta}$ (with respect to the $L^{2}$-inner product determined by $w$ ) if and only if $\eta=w^{-2} \zeta$, where

$$
w^{-1} \zeta \in W_{\mathbb{A}}^{k-1, p}\left(X, \Lambda^{2,+} \mathrm{T}^{*} X \otimes \mathfrak{g}\right), \quad\left(\mathrm{d}_{\mathbb{A}}+\mathrm{d} X_{f}(\mathbb{A})\right)^{*} \zeta=0 .
$$

The subscript in $W_{\mathbb{A}}^{k-1, p}$ indicates the dual boundary condition. It follows from linear exponential decay in Theorem 5.9 that every solution $\zeta$ of (69) decays exponentially. Hence the space of solutions of (69) is independent of the choice of the weight function $w$ as long as the $\left|\delta_{j}\right|$ are sufficiently small. This proves the theorem.

Remark 6.10 (i) The linearized operator is gauge equivariant in the sense that $\mathcal{D}_{u^{*} \mathbb{A}, \delta}\left(u^{-1} \alpha u\right)=u^{-1}\left(\mathcal{D}_{\mathbb{A}, \delta} \alpha\right) u$ for all $\alpha \in W_{\mathbb{A}, \delta}^{k, p}\left(X, \mathrm{~T}^{*} X \otimes \mathfrak{g}\right)$ and all gauge transformations $u \in \mathcal{G}(X)$ that satisfy $u \circ \iota_{j} \equiv u_{j} \in \mathcal{G}\left(Y_{j}\right)$.

(ii) In contrast to Theorem 6.9 (iv), the kernel of $\mathcal{D}_{\mathbb{A}, \delta}$ is not independent of the sign of the $\delta_{j}$ unless the $A_{j}$ are also irreducible.

(iii) On a tube $X=\mathbb{R} \times Y$ we will use weight functions of the form

$$
w(s, y)=\exp (V(s))
$$


with $V \in \mathcal{C}^{\infty}(\mathbb{R})$ such that $V(s)= \pm \delta s$ for $\pm s \geq 1$ (ie $\delta_{1}=\delta_{2}=: \delta>0$ ). Then $\mathcal{D}_{\mathbb{A}, \delta}$ can - as in Section 3 - be identified with the operator

$$
\begin{aligned}
\mathcal{D}_{\mathbb{A}, \delta}: W_{\mathbb{A}, \delta}^{k, p}\left(\mathbb{R} \times Y, \mathrm{~T}^{*} Y \otimes\right. & \mathfrak{g}) \times W_{\delta}^{k, p}(\mathbb{R} \times Y, \mathfrak{g}) \\
\rightarrow & W_{\delta}^{k-1, p}\left(\mathbb{R} \times Y, \mathrm{~T}^{*} Y \otimes \mathfrak{g}\right) \times W_{\delta}^{k-1, p}(\mathbb{R} \times Y, \mathfrak{g})
\end{aligned}
$$

given by $\quad \mathcal{D}_{\mathbb{A}, \delta}:=\nabla_{s}+\mathcal{H}_{A(s)}+\left(\begin{array}{cc}0 & 0 \\ 0 & 2 \lambda\end{array}\right), \quad \lambda:=\partial_{s} V$.

The formal $L_{\delta}^{2}$-adjoint operator of $\mathcal{D}_{\mathbb{A}, \delta}$ has the form

$$
\mathcal{D}_{\mathbb{A}, \delta}^{*}(\alpha, \varphi):=-\nabla_{s}+\mathcal{H}_{A(s)}-\left(\begin{array}{cc}
2 \lambda & 0 \\
0 & 0
\end{array}\right) .
$$

(iv) The operator (71) is conjugate to the operator

$$
w \mathcal{D}_{\mathbb{A}, \delta} w^{-1}=\nabla_{s}+\mathcal{H}_{A(s)}-I_{\lambda(s)}, \quad I_{\lambda}:=\left(\begin{array}{cc}
\lambda & 0 \\
0 & -\lambda
\end{array}\right),
$$

on the unweighted Sobolev spaces. By Theorem 6.9 (iv) and its proof, this operator is surjective if and only if the operator $\nabla_{S}+\mathcal{H}_{A(s)}-I_{\delta}$ is surjective, provided $\delta \in \mathbb{R} \backslash\{0\}$ is sufficiently small and $\mathbb{A} \in \widetilde{\mathcal{M}}\left(A^{-}, A^{+} ; X_{f}\right)$ is a Floer connecting trajectory with nondegenerate ends.

\section{The nonlinear setup}

In the remainder of this section we fix the constants $\delta_{1}=\cdots=\delta_{n}=\delta>0$. Then the operators $\mathcal{D}_{\mathbb{A}, \delta}$ have the following significance for the study of the moduli space $\mathcal{M}\left(A_{1}, \ldots, A_{n} ; X_{f}\right)$. Let $\mathbb{A} \in \widetilde{\mathcal{M}}\left(A_{1}, \ldots, A_{n} ; X_{f}\right)$ and suppose that $\mathcal{D}_{\mathbb{A}, \delta}$ is surjective. If the $A_{j}$ are all nondegenerate and irreducible and $\delta=0$, then $\mathcal{M}\left(A_{1}, \ldots, A_{n} ; X_{f}\right)$ is a smooth manifold near $[\mathbb{A}]$ whose tangent space is the kernel of $\mathcal{D}_{\mathbb{A}}=\mathcal{D}_{\mathbb{A}, \delta}$. In general, the kernel of $\mathcal{D}_{\mathbb{A}, \delta}$ is the tangent space of the quotient

$$
\mathcal{M}_{0}\left(A_{1}, \ldots, A_{n} ; X_{f}\right):=\widetilde{\mathcal{M}}\left(A_{1}, \ldots, A_{n} ; X_{f}\right) / \mathcal{G}_{0}(X),
$$

where $\mathcal{G}_{0}(X)$ denotes the group of gauge transformations $u \in \mathcal{G}(X)$ that satisfy $u \circ \iota_{j} \equiv \mathbb{1}$ for every $j$. Hence the dimension of $\mathcal{M}\left(A_{1}, \ldots, A_{n} ; X_{f}\right)$ is equal to

$$
\delta_{f}(\mathbb{A}):=\operatorname{index} \mathcal{D}_{\mathbb{A}, \delta}-\sum_{j=1}^{n} \operatorname{dim} H_{A_{j}}^{0} .
$$

(This agrees with the notation in Theorem 6.7.) To prove these assertions one can set up the nonlinear theory as follows. Fix an integer $k \geq 1$ and a real number $p>2$. 
Associated to a tuple $A_{j} \in \mathcal{A}\left(Y_{j}, \mathcal{L}\right), j=1, \ldots, n$, of critical points of the perturbed Chern-Simons functionals is a Banach manifold

$$
\mathcal{A}_{\delta}^{k, p}\left(X, \mathcal{L} ; A_{1}, \ldots, A_{n}\right):=\left\{\begin{array}{l|l}
\mathbb{A}=\mathbb{A}_{0}+\alpha & \begin{array}{l}
\alpha \in W_{\delta}^{k, p}\left(X, \mathrm{~T}^{*} X \otimes \mathfrak{g}\right) \\
\tau_{i, s}^{*} \mathbb{A} \in \mathcal{L}_{i} \forall i \forall s \in \mathcal{S}_{i}
\end{array}
\end{array}\right\}
$$

where $\mathbb{A}_{0} \in \mathcal{A}(X, \mathcal{L})$ is a reference connection satisfying $\iota_{j}^{*} \mathbb{A}_{0} \equiv A_{j}$ for all $j$. The tangent space of $\mathcal{A}_{\delta}^{k, p}\left(X, \mathcal{L} ; A_{1}, \ldots, A_{n}\right)$ is

$$
\mathrm{T}_{\mathbb{A}} \mathcal{A}_{\delta}^{k, p}\left(X, \mathcal{L} ; A_{1}, \ldots, A_{n}\right)=\left\{\alpha \in W_{\delta}^{k, p}\left(X, \mathrm{~T}^{*} X \otimes \mathfrak{g}\right) \mid \tau_{i, s}^{*} \alpha \in \mathrm{T}_{\tau_{i, s}^{*} \mathbb{A}} \mathcal{L}_{i}\right\} .
$$

Banach submanifold charts for $\mathcal{A}_{\delta}^{k, p}\left(X, \mathcal{L} ; A_{1} \ldots A_{n}\right) \subset \mathbb{A}_{0}+W_{\delta}^{k, p}\left(X, \mathrm{~T}^{*} X \otimes \mathfrak{g}\right)$ can be constructed with the help of the Banach submanifold coordinates for $\mathcal{L}_{i} \subset$ $\mathcal{A}^{0, p}\left(\Sigma_{i}\right)$ in [33, Lemma 4.3] (see Appendix E). The gauge group

$$
\mathcal{G}_{\delta}^{k+1, p}(X):=\left\{u: X \rightarrow \mathrm{G} \mid u^{-1} d u \in W_{\delta}^{k, p}(X, \mathfrak{g}), \lim _{s \rightarrow \infty} u \circ \iota_{j}=\mathbb{1}\right\}
$$

acts freely on $\mathcal{A}_{\delta}^{k, p}\left(X, \mathcal{L} ; A_{1}, \ldots, A_{n}\right)$. Its Lie algebra is the Banach space $W_{\delta}^{k+1, p}(X, \mathfrak{g})$ and the quotient $\mathcal{A}_{\delta}^{k, p}\left(X, \mathcal{L} ; A_{1}, \ldots, A_{n}\right) / \mathcal{G}_{\delta}^{k+1, p}(X)$ is a Banach manifold. There is a gauge equivariant smooth map

$$
\mathcal{A}_{\delta}^{k, p}\left(X, \mathcal{L} ; A_{1}, \ldots, A_{n}\right) \rightarrow W_{\delta}^{k, p}\left(X, \Lambda^{2,+} \mathrm{T}^{*} X \otimes \mathfrak{g}\right): \mathbb{A} \mapsto\left(F_{\mathbb{A}}+X_{f}(\mathbb{A})\right)^{+}
$$

and the moduli space $\mathcal{M}_{0}\left(A_{1}, \ldots, A_{n} ; X_{f}\right)$ can be identified with the quotient of the zero set of this map by the action of $\mathcal{G}_{\delta}^{k+1, p}(X)$. The operator $\mathcal{D}_{\mathbb{A}, \delta}$ arises from linearizing this setup in a local slice of the gauge group action and hence, if this operator is surjective, it follows from the implicit function theorem that $\mathcal{M}_{0}\left(A_{1}, \ldots, A_{n} ; X_{f}\right)$ is a smooth manifold near $\mathbb{A}$, whose tangent space can be identified with the kernel of $\mathcal{D}_{\mathbb{A}, \delta}$. The isotropy group $\mathcal{G}_{A_{1}} \times \cdots \times \mathcal{G}_{A_{n}}$ still acts on $\mathcal{M}_{0}\left(A_{1}, \ldots, A_{n} ; X_{f}\right)$ and the quotient by this action is the moduli space $\mathcal{M}\left(A_{1}, \ldots, A_{n} ; X_{f}\right)$. If all limit connections $A_{j}$ are irreducible then the action is free, so the moduli space is smooth.

\section{The spectral flow}

We now specialize to the case $X:=\mathbb{R} \times Y$ and establish index identities for the linearized operator. The main results are Theorem 6.11 and Corollary 6.14 below. They will be proven by identifying the index with a spectral flow.

We fix a gauge invariant, monotone Lagrangian submanifold $\mathcal{L} \subset \mathcal{A}(\partial Y)$ satisfying (L1)(L2) such that the zero connection is contained in $\mathcal{L}$ and is nondegenerate. Choose a perturbation $h_{f}: \mathcal{A}(Y) \rightarrow \mathbb{R}$ as in the introduction with a conjugation invariant function $f: \mathbb{D} \times \mathrm{G}^{N} \rightarrow \mathbb{R}$. Then the zero connection is a (nondegenerate) critical point of the perturbed Chern-Simons functional. For $A \in \operatorname{Crit}\left(\mathcal{C S}_{\mathcal{L}}+h_{f}\right)$ and a path $B:[0,1] \rightarrow \mathcal{L}$ 
from $B(0)=\left.A\right|_{\Sigma}$ to $B(1)=0$ we define an integer $\mu_{f}(A, B)$ as follows. Choose a smooth path $A:[0,1] \rightarrow \mathcal{A}(Y, \mathcal{L})$ such that $A(0)=A, A(1)=0$, and $\left.A(s)\right|_{\Sigma}=B(s)$. Define

$$
\mu_{f}(A, B):=\mu_{\text {spec }}\left(\left\{\mathcal{H}_{A(s)}+I_{\varepsilon}\right\}_{s \in[0,1]}\right), \quad I_{\varepsilon}:=\left(\begin{array}{cc}
\varepsilon & 0 \\
0 & -\varepsilon
\end{array}\right),
$$

where $\mu_{\text {spec }}$ denotes the upward spectral flow (see eg Robbin and Salamon [26] and Appendix A) and $\varepsilon>0$ is sufficiently small. This integer is independent of the choice of the path $A$ and the constant $\varepsilon$ used to define it. (The space of paths $A$ with fixed endpoints and boundary values is in fact convex. Moreover, the kernel ker $\mathcal{H}_{A}=H_{A, f}^{1} \times H_{A}^{0}$ splits at the endpoints $A=A(0), A(1)$ by Proposition 3.1.)

The significance of the following theorem is that the index resp. local dimension of the moduli space $\mathcal{M}\left(A^{-}, A^{+}\right)$is determined modulo 8 by the limit connections $A^{-}, A^{+}$.

Theorem 6.11 (i) Let $A^{ \pm} \in \mathcal{A}(Y, \mathcal{L})$ be critical points of $\mathcal{C} \mathcal{S}_{\mathcal{L}}+h_{f}$ and $\mathbb{A} \in \mathcal{A}(\mathbb{R} \times Y)$ be the connection associated to a smooth path $A: \mathbb{R} \rightarrow \mathcal{A}(Y, \mathcal{L})$ with limits

$$
\lim _{s \rightarrow \pm \infty}\left\|A-A^{ \pm}\right\|_{\mathcal{C}^{1}([s-1, s+1] \times Y)}=0 .
$$

Choose paths $B^{ \pm}:[0,1] \rightarrow \mathcal{L}$ from $B^{ \pm}(0)=\left.A^{ \pm}\right|_{\Sigma}$ to $B^{ \pm}(1)=0$ such that $B^{-}$is homotopic to the catenation of the path $\mathbb{R} \rightarrow \mathcal{L}:\left.s \mapsto A(s)\right|_{\Sigma}$ with $B^{+}$. Then

$$
\text { index } \mathcal{D}_{\mathbb{A}, \delta}=\mu_{\text {spec }}\left(\left\{\mathcal{H}_{A(s)}-I_{\lambda(s)}\right\}_{s \in \mathbb{R}}\right)
$$

and moreover

$$
\begin{aligned}
\delta_{f}(\mathbb{A}) & :=\operatorname{index} \mathcal{D}_{\mathbb{A}, \delta}-\operatorname{dim} H_{A^{-}}^{0}-\operatorname{dim} H_{A^{+}}^{0} \\
& =\mu_{f}\left(A^{-}, B^{-}\right)-\mu_{f}\left(A^{+}, B^{+}\right)-\operatorname{dim} H_{A^{-}}^{0}-\operatorname{dim} H_{A^{+}, f}^{1} .
\end{aligned}
$$

(ii) If $A \in \mathcal{A}(Y, \mathcal{L})$ is a critical point of $\mathcal{C S}_{\mathcal{L}}+h_{f}$ and $B:[0,1] \rightarrow \mathcal{L}$ is a path from $B(0)=\left.A\right|_{\Sigma}$ to $B(1)=0$, then for every loop $u:[0,1] \rightarrow \mathcal{G}(\Sigma)$ with $u(0)=u(1)=\mathbb{1}$

$$
\mu_{f}(A, B)-\mu_{f}\left(A, u^{*} B\right)=8 \operatorname{deg} u .
$$

Proof Multiplication by $w$ defines an isomorphism $W_{\delta}^{k, p} \rightarrow W^{k, p}$, so $\mathcal{D}_{\mathbb{A}, \delta}$ has the same index as the operator $w \mathcal{D}_{\mathbb{A}, \delta} w^{-1}$ on $W_{\mathbb{A}}^{k, p}\left(\mathbb{R} \times Y, \mathrm{~T}^{*}(\mathbb{R} \times Y) \otimes \mathfrak{g}\right)$. Hence, by (72) and Theorem A.4, the index of the operator $\mathcal{D}_{\mathbb{A}, \delta}$ is given by

$$
\begin{aligned}
\operatorname{index}\left(\mathcal{D}_{\mathbb{A}, \delta}\right) & =\mu_{\text {spec }}\left(\left\{\mathcal{H}_{A(s)}-I_{\lambda(s)}\right\}_{s \in \mathbb{R}}\right) \\
& =\mu_{\text {spec }}\left(\left\{\mathcal{H}_{A(s)}+I_{\delta}\right\}_{s \in \mathbb{R}}\right)-\operatorname{dim} H_{A^{+}, f}^{1}+\operatorname{dim} H_{A^{+}}^{0} .
\end{aligned}
$$


Here $\lambda:=\partial_{s} V: \mathbb{R} \rightarrow \mathbb{R}$ satisfies $\lambda(s)=-\delta$ for $s \leq-1$ and $\lambda(s)=\delta$ for $s \geq 1$. The second equation follows from a homotopy argument. Namely, the path $\mathcal{H}_{A(s)}-I_{\lambda(s)}$ is homotopic to the catenation of the path $\mathcal{H}_{A(s)}+I_{\delta}$ with $\mathcal{H}_{A^{+}}-I_{\lambda(s)}$. Now the catenation of the path $\mathcal{H}_{A(s)}+I_{\varepsilon}$ with the path in the definition of $\mu_{f}\left(A^{+}, B^{+}\right)$yields a path homotopic to the one in the definition of $\mu_{f}\left(A^{-}, B^{-}\right)$. (By assumption the paths are homotopic over the boundary $\partial Y$, and this homotopy can be extended to the interior.) Hence

$$
\mu_{f}\left(A^{-}, B^{-}\right)=\mu_{\text {spec }}\left(\left\{\mathcal{H}_{A(s)}+I_{\varepsilon}\right\}_{s \in \mathbb{R}}\right)+\mu_{f}\left(A^{+}, B^{+}\right) .
$$

For $\delta>0$ sufficiently small we can choose $\varepsilon=\delta$ and obtain

$$
\mu_{f}\left(A^{-}, B^{-}\right)-\mu_{f}\left(A^{+}, B^{+}\right)=\operatorname{index}\left(\mathcal{D}_{\mathbb{A}, \delta}\right)-\operatorname{dim} H_{A^{+}}^{0}+\operatorname{dim} H_{A^{+}, f}^{1} .
$$

This proves (i).

To prove (ii) choose a path $A(s):[0,1] \rightarrow \mathcal{A}(Y, \mathcal{L})$ with $A(0)=A, A(1)=0$, and $B(s)=\left.A(s)\right|_{\Sigma}$. By homotopy invariance we may assume that $A(s)=0$ for $s \geq$ $1 / 2$. Now let $u:[0,1] \rightarrow \mathcal{G}(\Sigma)$ be a loop with $u(0)=u(1)=\mathbb{1}$ and choose a path $A^{\prime}:[0,1] \rightarrow \mathcal{A}(Y, \mathcal{L})$ such that $A^{\prime}(0)=A, A^{\prime}(1)=0$ and $\left.A^{\prime}(s)\right|_{\Sigma}=u(s)^{*} B(s)$. Assume w.l.o.g. that $u(s)=11$ and $A^{\prime}(s)=A(s)$ for $s \leq 1 / 2$. Then the spectral flow of the path $\mathcal{H}_{A^{\prime}(s)}+I_{\varepsilon}$ on the interval $0 \leq s \leq 1 / 2$ is equal to $\mu_{f}(A, B)$. On the other hand, by Theorem A.5 and a homotopy from $\mathcal{H}_{A^{\prime}}+I_{\varepsilon}$ to $\mathcal{H}_{A^{\prime}}$, the spectral flow on the interval $1 / 2 \leq s \leq 1$ is equal to index $\left(\mathcal{D}_{\mathbb{1}, \mathbb{A}}\right)$ for a connection $\mathbb{A}=\tilde{u}^{-1} \mathrm{~d} \tilde{u} \in \mathcal{A}\left(S^{1} \times Y, \mathcal{L}\right)$ on the bundle $P_{\mathbb{1}}$ in the notation of Section 4. Here $\tilde{u} \in \mathcal{G}\left(S^{1} \times Y\right)$ is homotopic to $u$ on $[1 / 2,1] \times Y$ and identically $\mathbb{1 l}$ on the complement. Hence

$$
\begin{aligned}
\mu_{f}(A, B)-\mu_{f}\left(A, u^{*} B\right) & =-\mu_{\text {spec }}\left(\left\{\mathcal{H}_{A^{\prime}(s)}+I_{\varepsilon}\right\}_{1 / 2 \leq s \leq 1}\right) \\
& =-\operatorname{index}\left(\mathcal{D}_{\mathbb{1}, \mathbb{A}}\right)=8 \operatorname{deg}(\mathbb{1 1}, \mathbb{A})=8 \operatorname{deg}(u) .
\end{aligned}
$$

Here the third identity follows from Theorem 4.3 (ii) and the last from Remark 4.2 (iii). This proves the theorem.

For every critical point $A \in \mathcal{A}(Y, \mathcal{L})$ of the perturbed Chern-Simons functional we define the real number $\eta_{f}(A)$ by

$$
\eta_{f}(A):=\mu_{f}(A, B)-\frac{2}{\pi^{2}}\left(\mathcal{C S}(A, B)+h_{f}(A)\right),
$$

where $B:[0,1] \rightarrow \mathcal{L}$ is a path from $B(0)=\left.A\right|_{\Sigma}$ to $B(1)=0$, and $\mathcal{C S}(A, B)$ denotes the value of the Chern-Simons functional for the connection given by $A$ and $B$.

Corollary 6.12 (i) The spectral flow $(A, B) \mapsto \mu_{f}(A, B)$ descends to a circle valued function $\mu_{f}: \mathcal{R}_{f} \rightarrow \mathbb{Z} / 8 \mathbb{Z}$. 
(ii) The function $\eta_{f}$ : $\operatorname{Crit}\left(\mathcal{C S}_{\mathcal{L}}+h_{f}\right) \rightarrow \mathbb{R}$ is well defined and descends to a real valued function on $\mathcal{R}_{f}$.

Proof Lemma 2.4 (iii), the homotopy invariance of the spectral flow, and Theorem 6.11 (ii) imply that $\mu_{f}(A, B) \in \mathbb{Z} / 8 \mathbb{Z}$ is independent of the choice of $B$. Given a gauge transformation $u \in \mathcal{G}(Y)$ we can connect it to the identity by a smooth path $\tilde{u}:[0,1] \rightarrow \mathcal{G}(Y)$ from $\tilde{u}(0)=u$ to $\tilde{u}(1)=\mathbb{1}$. Let $A:[0,1] \rightarrow \mathcal{A}(Y, \mathcal{L})$ be the path in the definition of $\mu_{f}(A, B)$, then $\mu_{f}\left(u^{*} A,\left(\left.\tilde{u}\right|_{\partial Y}\right)^{*} B\right)$ is defined as the spectral flow along the path $s \mapsto \widetilde{u}(s)^{*} A(s)$ and hence, by the gauge equivariance of the Hessian,

$$
\mu_{f}(A, B)=\mu_{f}\left(u^{*} A,\left(\left.\widetilde{u}\right|_{\partial Y}\right)^{*} B\right) .
$$

This proves (i). That $\eta_{f}$ is well defined (ie independent of the choice of $B$ ) follows from Lemma 2.4 and Theorem 6.11 (ii). To see that $\eta_{f}$ is gauge invariant it remains to check that

$$
\mathcal{C S}(A, B)=\mathcal{C S}\left(u^{*} A,\left(\left.\widetilde{u}\right|_{\partial Y}\right)^{*} B\right) .
$$

This follows from the same argument as Lemma 2.4 (iv). Namely, $\mathcal{C S}(A, B)$ is the Chern-Simons functional on $\tilde{Y}=Y \cup([0,1] \times \Sigma)$ of a connection $\tilde{A}$ given by $A$ and $B$. The connection given by $u^{*} A$ and $\left(\left.\widetilde{u}\right|_{\partial Y}\right)^{*} B$ is $\hat{u}^{*} \widetilde{A}$, where the gauge transformation $\hat{u} \in \mathcal{G}(\tilde{Y})$ is given by $u$ and $\left.\tilde{u}\right|_{\partial Y}$. It satisfies $\left.\hat{u}\right|_{\partial Y}=11$ and has degree zero since a homotopy to $\mathbb{1}$ is given by combining $\widetilde{u}(\sigma)$ on $Y$ with $\left.s \mapsto \widetilde{u}(s+(1-s) \sigma)\right|_{\partial Y}$ on $[0,1] \times \Sigma$. Hence the equality of the Chern-Simons functionals follows from the analogue of (5) for manifolds with boundary and gauge transformations that are trivial on the boundary.

Remark 6.13 The function $(f, A) \mapsto \eta_{f}(A)$ is continuous on the space of nondegenerate pairs $(f, A)$. To see this note that the dimension of $H_{A}^{0}$ cannot jump, by Remark 3.8 , and hence one can locally work with the same constant $\varepsilon>0$ for the definition of $\mu_{f}$ in a neighbourhood of a pair $(f, A)$.

We can now state further index identities. The monotonicity formula in (i) below - a linear relationship between index and energy - will be central for excluding bubbling effects.

Corollary 6.14 (i) Let $\mathbb{A} \in \mathcal{A}(\mathbb{R} \times Y)$ be the connection associated to a smooth solution $A: \mathbb{R} \rightarrow \mathcal{A}(Y, \mathcal{L})$ of (14). Suppose that it satisfies (76) with the critical points $A^{ \pm} \in \mathcal{A}(Y, \mathcal{L})$ of $\mathcal{C} \mathcal{S}_{\mathcal{L}}+h_{f}$. Then

$$
\delta_{f}(\mathbb{A})=\frac{2}{\pi^{2}} E_{f}(\mathbb{A})+\eta_{f}\left(A^{-}\right)-\eta_{f}\left(A^{+}\right)-\operatorname{dim} H_{A^{-}}^{0}-\operatorname{dim} H_{A^{+}, f}^{1} .
$$


(ii) If $A, A^{\prime}: \mathbb{R} \rightarrow \mathcal{A}(Y, \mathcal{L})$ are paths connecting $A^{-}$to $B$, respectively $B$ to $A^{+}$, then the index of their catenation is given by

$$
\delta_{f}\left(\mathbb{A} \# \mathbb{A}^{\prime}\right)=\delta_{f}(\mathbb{A})+\delta_{f}\left(\mathbb{A}^{\prime}\right)+\operatorname{dim} H_{B}^{0}+\operatorname{dim} H_{B, f}^{1} .
$$

(iii) If $A: \mathbb{R} \rightarrow \mathcal{A}(Y, \mathcal{L})$ is a self-connecting path with limits $A^{-}=A^{+}=: A_{0}$ and $\left.s \mapsto A(s)\right|_{\Sigma}$ is homotopic to $\left.s \mapsto u(s)^{*} A_{0}\right|_{\Sigma}$ for $u: \mathbb{R} \rightarrow \mathcal{G}(\Sigma)$ with $u( \pm \infty)=\mathbb{1}$, then

$$
\delta_{f}(\mathbb{A})=8 \operatorname{deg}(u)-\operatorname{dim} H_{A_{0}}^{0}-\operatorname{dim} H_{A_{0}, f}^{1}
$$

Proof Assertions (ii) and (iii) follow immediately from Theorem 6.11. Assertion (i) follows from the definition of $\eta_{f}$, Theorem 6.11, and the following energy identity. For a path $A: \mathbb{R} \rightarrow \mathcal{A}(Y, \mathcal{L})$ satisfying

$$
\partial_{s} A=-*\left(F_{A}+X_{f}(A)\right)
$$

choose paths $B^{ \pm}:[0,1] \rightarrow \mathcal{L}$ from $B^{ \pm}(0)=\left.A^{ \pm}\right|_{\Sigma}$ to $B^{ \pm}(1)=0$ such that $B^{-}$is homotopic to the catenation of $\left.A(s)\right|_{\Sigma}$ with $B^{+}$. Then

$$
\begin{aligned}
-E_{f}(\mathbb{A})= & \int_{\mathbb{R}} \int_{Y}\left\langle\partial_{s} A \wedge\left(F_{A}+X_{f}(A)\right)\right\rangle \mathrm{d} s \\
= & \int_{\mathbb{R}}\left(\frac{1}{2} \frac{\partial}{\partial s} \int_{Y}\left(\langle A \wedge \mathrm{d} A\rangle+\frac{1}{3}\langle A \wedge[A \wedge A]\rangle\right)\right. \\
& \left.\quad+\frac{1}{2} \int_{\Sigma}\left\langle A \wedge \partial_{s} A\right\rangle+\frac{\partial}{\partial s} h_{f}(A)\right) \mathrm{d} s \\
= & \mathcal{C S}\left(A^{+}, B^{+}\right)+h_{f}\left(A^{+}\right)-\mathcal{C S}\left(A^{-}, B^{-}\right)-h_{f}\left(A^{-}\right) .
\end{aligned}
$$

Here the second equation follows from (9) and the fact that

$$
2 \int_{Y}\left\langle F_{A} \wedge \partial_{S} A\right\rangle=\int_{Y} \frac{\partial}{\partial s}\left(\langle A \wedge \mathrm{d} A\rangle+\frac{1}{3}\langle A \wedge[A \wedge A]\rangle\right)+\int_{\partial Y}\left\langle A \wedge \partial_{S} A\right\rangle .
$$

The last identity follows from the $\mathcal{C}^{1}$-convergence of $A$ for $s \rightarrow \pm \infty$. Since $B^{-}$is homotopic (with fixed endpoints) to the catenation of $\left.A\right|_{\Sigma}$ with $B^{+}$, we have

$$
\int_{\mathbb{R}} \int_{\Sigma}\left\langle A \wedge \partial_{S} A\right\rangle \mathrm{d} s=\int_{0}^{1} \int_{\Sigma}\left\langle B^{-} \wedge \partial_{s} B^{-}\right\rangle \mathrm{d} s-\int_{0}^{1} \int_{\Sigma}\left\langle B^{+} \wedge \partial_{s} B^{+}\right\rangle \mathrm{d} s .
$$

(See the proof of Lemma 2.4 above for the invariance of this integral under homotopy.) This proves the corollary.

Remark 6.15 Our notation for the indices is motivated by the following finite dimensional model. Let $M$ be a Riemannian $n$-manifold, $\mathrm{G}$ be a compact Lie group that 
acts on $M$ by isometries, and $f: M \rightarrow \mathbb{R}$ be a G-invariant Morse-Bott function. Associated to every critical point $x \in M$ is a chain complex

$$
0 \longrightarrow \mathfrak{g} \stackrel{L_{x}}{\longrightarrow} \mathrm{T}_{x} M \stackrel{\nabla^{2} f(x)}{\longrightarrow} \mathrm{T}_{x} M \stackrel{L_{x}^{*}}{\longrightarrow} \mathfrak{g} \longrightarrow 0,
$$

where $L_{x}$ is the infinitesimal action of $\mathfrak{g}$ and $\nabla^{2} f(x)$ is the Hessian of $f$ (see (12)). We denote

$$
v_{0}(x):=\operatorname{dim} \operatorname{ker} L_{x}, \quad v_{1}(x):=\operatorname{dim} \frac{\operatorname{ker} \nabla^{2} f(x)}{\operatorname{im} L_{x}}, \quad \mu(x):=\operatorname{ind}_{f}(x),
$$

that is $\mu(x)$ is the number of negative eigenvalues of the Hessian and $v_{0}(x)$ is the dimension of the isotropy subgroup. Now the kernel of the Hessian has dimension $v_{1}(x)+\operatorname{dim} \mathrm{G}-v_{0}(x)$, the unstable manifold $W^{u}(x)$ of the orbit $\mathrm{G} x$ has dimension $\mu(x)+\operatorname{dim} \mathrm{G}-v_{0}(x)$, the stable manifold $W^{s}(x)$ of $\mathrm{G} x$ has dimension $n-\mu(x)-v_{1}(x)$, and, in the transverse case, the moduli space

$$
\mathcal{M}\left(x^{-}, x^{+}\right):=W^{u}\left(x^{-}\right) \cap W^{s}\left(x^{+}\right) / \mathrm{G}
$$

of connecting trajectories has dimension (compare with (77))

$$
\delta\left(x^{-}, x^{+}\right):=\operatorname{dim} \mathcal{M}\left(x^{-}, x^{+}\right)=\mu\left(x^{-}\right)-\mu\left(x^{+}\right)-v_{0}\left(x^{-}\right)-v_{1}\left(x^{+}\right) .
$$

\section{Compactness}

Let $Y$ be a compact oriented Riemannian 3-manifold with boundary $\partial Y=\Sigma$ and $\mathcal{L} \subset$ $\mathcal{A}(\Sigma)$ be a gauge invariant, monotone, irreducible Lagrangian submanifold satisfying (L1)-(L2). Fix a collection of embeddings $\gamma_{i}: S^{1} \times \mathbb{D} \rightarrow \operatorname{int}(Y), i=1, \ldots, m$, as in Section 2. We use the notation

$$
\widetilde{\mathcal{M}}\left(A^{-}, A^{+} ; X_{f}\right):=\left\{\begin{array}{l|l}
\mathbb{A} \in \mathcal{A}^{\tau}(\mathbb{R} \times Y) & \begin{array}{l}
\partial_{s} A-\mathrm{d}_{A} \Phi+*\left(F_{A}+X_{f}(A)\right)=0, \\
\left.A(s)\right|_{\Sigma} \in \mathcal{L} \quad \forall s \in \mathbb{R}, \\
E_{f}(\mathbb{A})<\infty, \lim _{s \rightarrow \pm \infty} A(s)=A^{ \pm}
\end{array}
\end{array}\right\}
$$

for the space of Floer connecting trajectories associated to a perturbation $f \in \mathcal{C}^{\infty}(\mathbb{D} \times$ $\left.\mathrm{G}^{m}\right)^{\mathrm{G}}$ and two critical points $A^{ \pm} \in \mathcal{A}(Y, \mathcal{L})$ of $\mathcal{C} \mathcal{S}_{\mathcal{L}}+h_{f}$. Here $\mathcal{A}^{\tau}(\mathbb{R} \times Y)$ denotes the space of connections $\Xi=\Phi \mathrm{d} s+A$ on $\mathbb{R} \times Y$ that are in temporal gauge outside of $[-1,1] \times Y$, ie $\Phi(s)=0$ for $|s| \geq 1$. The corresponding gauge group $\mathcal{G}\left(A^{-}, A^{+}\right)$ consists of all gauge transformations $u: \mathbb{R} \rightarrow \mathcal{G}(Y)$ that satisfy $u(s)=u^{ \pm} \in \mathcal{G}_{A^{ \pm}}$for $\pm s \geq 1$ and the quotient space will be denoted by

$$
\mathcal{M}\left(A^{-}, A^{+} ; X_{f}\right):=\widetilde{\mathcal{M}}\left(A^{-}, A^{+} ; X_{f}\right) / \mathcal{G}\left(A^{-}, A^{+}\right) .
$$


The goal of this section is to establish compactness theorems for these moduli spaces. The proofs will be heavily based on the basic compactness results in [35; 36]. We start with a summary of the compactness for uniformly bounded curvature.

Proposition 7.1 Let $f^{\nu} \in \mathcal{C}^{\infty}\left(\mathbb{D} \times \mathrm{G}^{m}\right)^{\mathrm{G}}$ be a sequence that converges to $f^{\infty} \in$ $\mathcal{C}^{\infty}\left(\mathbb{D} \times \mathrm{G}^{m}\right)^{\mathrm{G}}$ in the $C^{k+1}$-topology for some $k \geq 1$. Let $I^{v} \subset \mathbb{R}$ be a sequence of open intervals such that $I^{v} \subset I^{v+1}$ for all $v$ and denote $I:=\bigcup_{v} I^{v}$. Let $\Xi^{v}=$ $\Phi^{v} \mathrm{~d} s+A^{v} \in \mathcal{A}\left(I^{v} \times Y\right)$ be a sequence of solutions of the Floer equation

$$
\partial_{s} A^{v}-\mathrm{d}_{A^{v}} \Phi^{v}+*\left(F_{A^{v}}+X_{f^{v}}\left(A^{v}\right)\right)=0,\left.\quad A^{v}(s)\right|_{\Sigma} \in \mathcal{L},
$$

such that the curvature $\left|F_{\Xi^{v}}\right|$ is locally uniformly bounded. Then the following holds.

(i) There exists a subsequence, still denoted by $\Xi^{v}$, and a sequence of gauge transformations $u^{v} \in \mathcal{G}\left(I^{v} \times Y\right)$ such that $\left(u^{v}\right)^{*} \Xi^{v}$ converges in the $\mathcal{C}^{k}$ topology on every compact subset of $I \times Y$.

(ii) There exists a subsequence, still denoted by $\Xi^{v}$, and a sequence of gauge transformations $u^{v} \in \mathcal{G}\left(I^{v} \times Y\right)$ such that $\left(u^{v}\right)^{*} \Xi^{v}$ is in temporal gauge and converges in the $\mathcal{C}^{k-1}$ topology on every compact subset of $I \times Y$.

(iii) In both cases, the limit $\Xi^{\infty} \in \mathcal{A}(I \times Y)$ of the subsequence can be chosen smooth and it satisfies (78) with $f^{\nu}$ replaced by $f^{\infty}$.

Proof In a neighbourhood of the boundary $I \times \partial Y$, where the perturbations vanish, compactness for anti-self-dual connections with Lagrangian boundary conditions was established in [35, Theorem B]. The interior compactness follows from standard techniques (eg Donaldson and Kronheimer [11] and Wehrheim [34]) and Remark D.2. The crucial point in the bootstrapping argument is that a $W^{k, p}$-bound on $\left(u^{v}\right)^{*} \Xi^{v}$ implies a $W^{k, p}$-bound on $X_{f^{v}}\left(\left(u^{v}\right)^{*} \Xi^{v}\right)$ and hence on $F_{\left(u^{v}\right)^{*} \Xi^{v}}^{+}$(The constant in the $W^{k, p}$-estimate of Proposition D.1 (iii) depends continuously on $f \in \mathcal{C}^{k+1}$.) Combining these two compactness results via a general patching procedure as in [11, Lemma 4.4.5] or [34, Proposition 7.6] we deduce that, for a suitable subsequence and choice of $u^{v}$, the sequence $\left(u^{v}\right)^{*} \Xi^{v}$ is bounded in $W^{k+1, p}(K)$ for every compact subset $K \subset I \times Y$ and a fixed $p>4$, and hence has a $\mathcal{C}^{k}$ convergent subsequence. A diagonal argument then proves (i).

To prove (ii) we write $\widetilde{\Xi}^{v}:=\left(u^{v}\right)^{*} \Xi^{v}=: \widetilde{\Phi}^{v} \mathrm{~d} s+\widetilde{A}^{v}$ where $u^{v}$ is as in (i). Then $\widetilde{\Xi}^{v}$ is bounded in $W^{k+1, p}$ on every compact subset of $I \times Y$. Define $v^{v}: I^{v} \times Y \rightarrow \mathrm{G}$ as the unique solution of the differential equation

$$
\partial_{s} v^{v}+\tilde{\Phi}^{v} v^{v}=0, \quad v^{v}(0)=1 .
$$


Then $v^{v}$ is bounded in $W^{k+1, p}$ on every compact subset of $I \times Y$. (To check this use the identity $\partial_{s}\left(v^{-1} \mathrm{~d} v\right)=-v^{-1} \Phi v$.) Hence $\left(v^{v}\right)^{*} \widetilde{\Xi}^{v}=\left(u^{v} v^{v}\right)^{*} \Xi^{v}$ is in temporal gauge and is bounded in $W^{k, p}$ on every compact subset of $I \times Y$. The compact embeddings $W^{k, p}(K) \hookrightarrow \mathcal{C}^{k-1}(K)$ together with a diagonal argument then prove (ii).

The regularity of the limit $\Xi^{\infty}$ can be achieved by a further gauge transformation. That $\Xi^{\infty}$ solves (78) follows from the fact that these equations are gauge invariant and preserved under weak $W^{k, p}$ convergence.

The following is the most general compactness result for bounded energy.

Theorem 7.2 Let $f \in \mathcal{C}^{\infty}\left(\mathbb{D} \times \mathrm{G}^{m}\right)^{\mathrm{G}}$ be a perturbation such that every critical point of $\mathcal{C} \mathcal{S}_{\mathcal{L}}+h_{f}$ is nondegenerate. Let $f^{v} \in \mathcal{C}^{\infty}\left(\mathbb{D} \times \mathrm{G}^{m}\right)^{\mathrm{G}}$ be a sequence that converges to $f$ in the $C^{k+1}$-topology and let $\Xi^{v}=\Phi^{v} \mathrm{~d} s+A^{v} \in \widetilde{\mathcal{M}}\left(A_{-}^{v}, A_{+}^{v} ; X_{f^{v}}\right)$ be a sequence of Floer connecting trajectories with bounded energy

$$
\sup _{\nu} E_{f^{v}}\left(\Xi^{v}\right)=\sup _{\nu} \int_{\mathbb{R} \times Y}\left|\partial_{s} A^{v}-\mathrm{d}_{A^{v}} \Phi^{v}\right|^{2}<\infty .
$$

Fix $p>1$ and suppose that $A_{ \pm}^{v}$ converges to $A^{ \pm} \in \operatorname{Crit}\left(\mathcal{C S}_{\mathcal{L}}+h_{f}\right)$ in the $\mathcal{C}^{k}$ topology. Then there is a subsequence, still denoted by $\Xi^{v}$, critical points $B_{0}, \ldots, B_{\ell} \in$ $\operatorname{Crit}\left(\mathcal{C S}_{\mathcal{L}}+h_{f}\right)$ with $B_{0}=A^{-}, B_{\ell}=A^{+}$, and Floer connecting trajectories $\Xi_{i} \in$ $\widetilde{\mathcal{M}}\left(B_{i-1}, B_{i} ; X_{f}\right)$ for $i=1, \ldots, \ell$, such that $\Xi^{v}$ converges to the broken trajectory $\left(\Xi_{1}, \ldots, \Xi_{\ell}\right)$ in the following sense.

For every $i \in\{1, \ldots, \ell\}$ there is a sequence $s_{i}^{v} \in \mathbb{R}$ and a sequence of gauge transformations $u_{i}^{v} \in \mathcal{G}(\mathbb{R} \times Y)$ such that the sequence $s \mapsto\left(\left(u_{i}^{v}\right)^{*} \Xi^{v}\right)\left(s+s_{i}^{v}\right)$ converges to $\Xi_{i}$ in the $W^{1, p}$-norm on every compact subset of $\mathbb{R} \times Y \backslash Z_{i}$. Here $Z_{i} \subset \mathbb{R} \times Y$ is the bubbling locus consisting of finitely many interior points and finitely many boundary slices; it is nonempty whenever $\Xi_{i}$ has zero energy.

The broken trajectory $\left(\Xi_{1}, \ldots, \Xi_{\ell}\right)$ has energy and index

$$
\begin{aligned}
& \sum_{i=1}^{\ell} E_{f}\left(\Xi_{i}\right) \leq \lim _{\nu \rightarrow \infty} E_{f^{\nu}}\left(\Xi^{\nu}\right), \\
& \sum_{i=1}^{\ell} \delta_{f}\left(\Xi_{i}\right)+\sum_{i=1}^{\ell-1} \operatorname{dim} H_{B_{i}}^{0} \leq \lim _{\nu \rightarrow \infty} \delta_{f^{\nu}}\left(\Xi^{\nu}\right) .
\end{aligned}
$$

If $\sup _{v}\left\|F_{\Xi^{v}}\right\|_{L^{\infty}}<\infty$ then there is no bubbling (ie $Z_{i}=\varnothing$ for all $i$ ), equality holds in (79), and $\left(u^{v}\right)^{*} \Xi^{v}$ converges in the $\mathcal{C}^{k}$ topology on every compact set. If 
$\sup _{v}\left\|F_{\Xi v}\right\|_{L^{\infty}}=\infty$ then there is bubbling (ie $Z_{i} \neq \varnothing$ for some $i$ ) and

$$
\begin{aligned}
& \sum_{i=1}^{\ell} E_{f}\left(\Xi_{i}\right) \leq \lim _{\nu \rightarrow \infty} E_{f^{\nu}}\left(\Xi^{v}\right)-4 \pi^{2} \\
& \sum_{i=1}^{\ell} \delta_{f}\left(\Xi_{i}\right)+\sum_{i=1}^{\ell-1} \operatorname{dim} H_{B_{i}}^{0} \leq \lim _{\nu \rightarrow \infty} \delta_{f^{\nu}}\left(\Xi^{\nu}\right)-8 .
\end{aligned}
$$

Remark 7.3 The assumption that $A_{ \pm}^{\nu}$ converges in the $\mathcal{C}^{k}$ topology always holds for a subsequence in a suitable gauge, by Proposition 3.7.

Proof of Theorem 7.2 Replacing the uniform bound on the curvature in Proposition 7.1 by an energy bound on $\Xi^{v}$ allows for bubbling. For the (unperturbed) antiself-duality equation with Lagrangian boundary conditions this was dealt with in [36, Theorems 1.2,1.5], [23], and [37, Section 3]; for the perturbed equation in the interior the (well known) result is Theorem D.4. Combining these one essentially obtains the same basic compactness theorem as for anti-self-dual connections (see [10, Proposition 2.1]). A minor difference is that - due to the holonomy perturbations - we obtain convergence in the $W^{1, p}$-norm for any $p>1$ rather than in the $\mathcal{C}^{\infty}$-topology; so [10, Proposition 2.1 (1)] is replaced by $W^{1, p}$-convergence. The crucial difference is in the knowledge about the bubbling phenomenon. First, the finite set $\left\{x_{1}, \ldots, x_{\ell}\right\} \subset$ $\mathbb{R} \times Y$ of bubbling points is replaced by a more general bubbling locus $Z \subset \mathbb{R} \times Y$ consisting of finitely many interior points and finitely many boundary slices $\{s\} \times \partial Y$. On the complement of $Z$, one has local $L^{p}$-bounds on the curvature. Second, we do not have a geometric description of the bubbles (after rescaling) or the precise quantum $4 \pi^{2}$ for the energy concentration. There is however a universal constant $\hbar>0$ that is a lower bound for the energy concentration at each component of the bubbling locus $Z$; so [10, Proposition 2.1 (2)] is replaced by $\int_{U}\left|F_{A}+X_{f}(A)\right|^{2} \leq \lim \sup _{\alpha^{\prime} \rightarrow \infty} \int_{U} \mid F_{A_{\alpha^{\prime}}}+$ $\left.X_{f_{\alpha^{\prime}}}\left(A_{\alpha^{\prime}}\right)\right|^{2}-\ell \hbar$, where $\ell$ is the number of points and boundary slices in $Z$.

The second source of noncompactness, the splitting of trajectories, is the same as for the usual Floer theories. With the exponential decay results of Section 5 and the modified basic compactness above, one can adapt the discussion in [10, Chapter 5.1] to prove the convergence to a broken trajectory. In particular, exponential decay holds for sufficiently $\mathcal{C}^{2}$-close perturbations with uniform constants (see Theorem 8.3 for the nondegeneracy and Proposition D.1 (v) for the constants). More precisely we argue as follows.

Throughout we denote the perturbed Yang-Mills energy of $\Xi^{v}$ on $I \times Y$ by

$$
E_{f^{v}}\left(\Xi^{v} ; I\right):=\int_{I} \int_{Y}\left|\partial_{s} A^{v}-\mathrm{d}_{A^{v}} \Phi^{v}\right|^{2}
$$


Passing to a subsequence we may assume that bubbling occurs only for finitely many sequences $t_{j}^{v}, j=1, \ldots, m$, with

$$
\hbar_{j}:=\lim _{\delta \rightarrow 0} \lim _{\nu \rightarrow \infty} E_{f^{v}}\left(\Xi^{v} ;\left[t_{j}^{v}-\delta, t_{j}^{v}+\delta\right]\right) \geq \hbar
$$

In particular, the limits exist. The sequences are chosen such that $t_{j+1}^{v}-t_{j}^{v}>0$ and that these differences converge either to a positive number or to infinity. We may also assume that the curvature of $\Xi^{v}$ is uniformly bounded on the complement of the sets $\left[t_{j}^{v}-\delta, t_{j}^{v}+\delta\right] \times Y$ for every $\delta>0$ and that the following limits exist:

$$
\begin{aligned}
\varepsilon_{0} & :=\lim _{\delta \rightarrow 0} \lim _{\nu \rightarrow \infty} E_{f^{v}}\left(\Xi^{v} ;\left(-\infty, t_{1}^{\nu}-\delta\right]\right), \\
\varepsilon_{j} & :=\lim _{\delta \rightarrow 0} \lim _{\nu \rightarrow \infty} E_{f^{v}}\left(\Xi^{v} ;\left[t_{j}^{v}+\delta, t_{j+1}^{v}-\delta\right]\right), \quad j=1, \ldots, m-1, \\
\varepsilon_{m} & :=\lim _{\delta \rightarrow 0} \lim _{\nu \rightarrow \infty} E_{f^{v}}\left(\Xi^{v} ;\left[t_{m}^{v}+\delta, \infty\right)\right) .
\end{aligned}
$$

Then

$$
\lim _{\nu \rightarrow \infty} E_{f^{v}}\left(\Xi^{\nu}\right)=\varepsilon_{0}+\hbar_{1}+\varepsilon_{1}+\cdots+\hbar_{m}+\varepsilon_{m}
$$

Next we choose a constant $\varepsilon>0$ smaller than the constant in Theorem 5.3 and smaller than $\hbar$. Following $[10,5.1]$ we choose the $s_{i}^{v} \in \mathbb{R}$ inductively such that

$$
E_{f^{v}}\left(\Xi^{v} ;\left(-\infty, s_{1}^{\nu}\right]\right)=\frac{\varepsilon}{2}, \quad E_{f^{v}}\left(\Xi^{v} ;\left[s_{i}^{v}, s_{i+1}^{\nu}\right]\right)=E_{f}\left(\Xi_{i}\right)+\sum_{j \in J_{i}} \hbar_{j},
$$

where $\Xi_{i}$ is the limit of the sequence $\Xi^{v}\left(s_{i}^{v}+\cdot\right)$ modulo gauge and bubbling and $J_{i} \subset\{1, \ldots, m\}$ denotes the set of all $j$ such that the sequence $t_{j}^{v}-s_{i}^{v}$ is bounded. This choice guarantees that $s_{i+1}^{\nu}-s_{i}^{v} \rightarrow \infty$ for all $i$, that $\{1, \ldots, m\}$ is the disjoint union of the $J_{i}$, and that $J_{i} \neq \varnothing$ whenever $\Xi_{i}$ has zero energy. By Theorem 5.3 (applied to a temporal gauge of the $\Xi^{v}$ on intervals $\left[s_{i}^{v}+T, s_{i+1}^{v}\right]$ with energy less than $\varepsilon$ ) the positive end of $\Xi_{i}$ is gauge equivalent (and hence w.l.o.g. equal to) the negative end of $\Xi_{i+1}$, the negative end of $\Xi_{1}$ is $A^{-}$, and the positive end of $\Xi_{\ell}$ is $A^{+}$. The total energy of the broken trajectory is

$$
\sum_{i=1}^{\ell} E_{f}\left(\Xi_{i}\right)=\sum_{j=0}^{m} \varepsilon_{j}=\lim _{\nu \rightarrow \infty} E_{f^{\nu}}\left(\Xi^{v}\right)-\sum_{j=1}^{m} \hbar_{j}
$$

If the curvature is bounded then $m=0$ and all bubbling loci $Z_{i}$ are empty. In this case the energy identity is (81) and the index identity follows from the monotonicity formula in Corollary 6.14 (i). If the curvature blows up then $m \geq 1$, hence $Z_{i} \neq \varnothing$ for 
some $i$, and we obtain the strict inequality

$$
\begin{aligned}
\sum_{i=1}^{\ell} \delta_{f}\left(\Xi_{i}\right)+\sum_{i=1}^{\ell} \operatorname{dim} H_{B_{i-1}}^{0} & =\sum_{i=1}^{\ell}\left(\frac{2}{\pi^{2}} E_{f}\left(\Xi_{i}\right)+\eta_{f}\left(B_{i-1}\right)-\eta_{f}\left(B_{i}\right)\right) \\
& <\lim _{\nu \rightarrow \infty}\left(\frac{2}{\pi^{2}} E_{f^{\nu}}\left(\Xi^{\nu}\right)+\eta_{f^{\nu}}\left(A_{-}^{\nu}\right)-\eta_{f^{\nu}}\left(A_{+}^{\nu}\right)\right) \\
& =\lim _{\nu \rightarrow \infty} \delta_{f^{v}}\left(\Xi^{v}\right)+\operatorname{dim} H_{A^{-}}^{0} .
\end{aligned}
$$

Here the first step follows from Corollary 6.14 (i), the second step uses (81) and the continuity of the function $(f, A) \mapsto \eta_{f}(A)$ (see Remark 6.13), and the last step uses Corollary 6.14 (ii) and $\operatorname{dim} H_{A^{v}}^{0}=\operatorname{dim} H_{A^{-}}^{0}$ for $v$ sufficiently large (see Remark 3.8). Each side of our inequality has the form $\delta_{f}(\Xi)+\operatorname{dim} H_{A^{-}}^{0}$ for a suitable path $\Xi$ running from $A^{-}$to $A^{+}$. For the left hand side, by Corollary 6.14 (ii), $\Xi$ can be chosen as the catenation of the $\Xi_{i}$ and for the right hand side as a small deformation of $\Xi^{v}$ for $v$ sufficiently large. Since the inequality is strict it follows from Theorem 6.11 (i) and Corollary 6.12 that the defect is at least 8 . Using monotonicity again we obtain an energy gap of at least $4 \pi^{2}$. This proves the theorem.

A first consequence of the compactness and index identities is that we can exclude bubbling in certain moduli spaces by transversality.

Corollary 7.4 Suppose that the sequence of solutions in Theorem 7.2 has index

$$
\delta_{f^{v}}\left(\Xi^{v}\right) \leq 7 .
$$

Suppose that either bubbling occurs or one of the limit trajectories $\Xi_{i}$ is a selfconnecting trajectory of $\left[B_{i-1}\right]=\left[B_{i}\right]=[0]$. Then one of the limit trajectories $\Xi_{j}$ must have negative index $\delta_{f}\left(\Xi_{j}\right)<0$ and at least one of its endpoints $B_{j-1}$ or $B_{j}$ is not gauge equivalent to the trivial connection.

Proof Every nontrivial self-connecting trajectory $\Xi_{i}$ of [0] has index $\delta_{f}\left(\Xi_{i}\right) \geq 5$ by Corollary 6.14 with $E_{f}\left(\Xi_{i}\right)=4 \pi^{2} \operatorname{deg}(u)>0$. It also adds $\operatorname{dim} H_{[0]}^{0}=3$ to the sum of indices. So to achieve a sum $\leq 7$, one of the other indices must be negative. A trivial self-connecting trajectory of [0] has index -3 but also adds another $\operatorname{dim} H_{[0]}^{0}=3$ to the sum of indices. Hence there must be a trajectory with negative index and at least one nontrivial end. The same holds in the bubbling case by (80).

We will refine the compactness theorem in two special cases. First we consider the case of no breaking and no bubbling in which we obtain actual compactness of moduli spaces. 
Theorem 7.5 Fix a constant $p>1$. Let $f, f^{\mathcal{v}}$ be as in Theorem 7.2 and $A^{ \pm} \in \mathcal{A}(Y, \mathcal{L})$ such that $F_{A^{ \pm}}+X_{f^{v}}\left(A^{ \pm}\right)=0$ for all $v$. Then there is a $\delta>0$ such that the following holds. If $\Xi \in \widetilde{\mathcal{M}}\left(A^{-}, A^{+} ; X_{f}\right)$ and, for each $v, \Xi^{v}$ is a solution of (78) that is gauge equivalent to an element of $\widetilde{\mathcal{M}}\left(A^{-}, A^{+} ; X_{f}\right)$ such that $\Xi^{v}$ converges to $\Xi$ in the $\mathcal{C}^{k}$ topology on compact sets and

$$
E_{f}(\Xi)=\lim _{\nu \rightarrow \infty} E_{f^{v}}\left(\Xi^{v}\right)
$$

then there exists a sequence of gauge transformations $u^{v} \in \mathcal{G}(\mathbb{R} \times Y)$ such that $\left(u^{v}\right)^{*} \Xi^{v}$ converges to $\Xi$ in $W_{\delta}^{k, p}(\mathbb{R} \times Y)$.

Proof Note that, by contradiction, it suffices to prove the convergence statement for a subsequence. For that purpose we choose $v^{v} \in \mathcal{G}(\mathbb{R} \times Y)$ such that

$$
\widetilde{\Xi}^{v}:=\left(v^{v}\right)^{*} \Xi^{v} \in \widetilde{\mathcal{M}}\left(A^{-}, A^{+} ; X_{f^{v}}\right) \text {. }
$$

In particular, $\widetilde{\Xi}^{v}=: \widetilde{\Phi}^{v} \mathrm{~d} s+\widetilde{A}^{v}$ is in temporal gauge outside of $[-1,1] \times Y$. Fix a constant $\varepsilon>0$ smaller than the constant in Corollary 5.4 and note that the exponential $\mathcal{C}^{k}$ estimate in Corollary 5.4 holds with uniform constants $\delta_{0}:=\delta>0$ and $C_{0}:=C_{k}$ in a sufficiently small $\mathcal{C}^{k+1}$ neighborhood of $f$. We write $\Xi=\Phi \mathrm{d} s+A$ and choose $T_{0}>0$ such that

$$
\int_{-T_{0}}^{T_{0}} \int_{Y}\left|\partial_{s} A-\mathrm{d}_{A} \Phi\right|^{2}>E_{f}(\Xi)-\varepsilon .
$$

Since $\Xi^{v}=\Phi^{v} \mathrm{~d} s+A^{v}$ converges in the $\mathcal{C}^{k}$ norm on compact sets we have

$$
\int_{-T_{0}}^{T_{0}} \int_{Y}\left|\partial_{S} \tilde{A}^{v}-\mathrm{d}_{\tilde{A}^{v}} \tilde{\Phi}^{v}\right|^{2}=\int_{-T_{0}}^{T_{0}} \int_{Y}\left|\partial_{S} A^{v}-\mathrm{d}_{A^{v}} \Phi^{v}\right|^{2}>E_{f^{v}}\left(\Xi^{v}\right)-\varepsilon
$$

and thus $E\left(\Xi^{v} ;\left(-\infty, T_{0}\right]\right)+E\left(\Xi^{v} ;\left[T_{0}, \infty\right)\right)<\varepsilon$ for sufficiently large $v \geq v_{0}$. Hence it follows from Corollary 5.4 that

$$
\begin{aligned}
\left\|\tilde{A}^{v}-A^{+}\right\|_{\mathcal{C}^{k}([s, \infty) \times Y)} & \leq C_{0} e^{-\delta_{0}\left(s-T_{0}\right)} E\left(\Xi^{v} ;\left[T_{0}, \infty\right)\right), \\
\left\|\tilde{A}^{v}-A^{-}\right\|_{\mathcal{C}^{k}((-\infty,-s] \times Y)} & \leq C_{0} e^{-\delta_{0}\left(s-T_{0}\right)} E\left(\Xi^{v} ;\left(-\infty,-T_{0}\right]\right)
\end{aligned}
$$

for $s \geq T_{0}+1$ and $v \geq v_{0}$. The same estimate holds with $\widetilde{A}^{v}$ replaced by $A$. Now fix a constant $0<\delta<\delta_{0}$. Then there exists a constant $C$ (depending on $C_{0}, \delta, \delta_{0}, k$, and $p$ ) such that

$$
\left\|\widetilde{\Xi}^{v}-\Xi\right\|_{W_{\delta}^{k, p}((\mathbb{R} \backslash[-T, T]) \times Y)} \leq C e^{-\left(\delta_{0}-\delta\right)\left(T-T_{0}\right)}
$$

for $T \geq T_{0}+1$ and $v \geq v_{0}$. 
Next, fix a sequence $\rho_{n} \rightarrow 0$ and choose $T_{n} \rightarrow \infty$ so that $T_{n} \geq T_{0}+1$ and

$$
C e^{-\left(\delta_{0}-\delta\right)\left(T_{n}-T_{0}\right)}<\frac{\rho_{n}}{2} .
$$

For fixed $n \in \mathbb{N}$ note that both $\Xi^{v}$ and $\widetilde{\Xi}^{v}=\left(v^{v}\right)^{*} \Xi^{v}$ converge to $\Xi$ in the $\mathcal{C}^{k}$ norm on $\left[T_{n}, T_{n}+1\right] \times Y$ and on $\left[-T_{n}-1,-T_{n}\right] \times Y$. Using the identity

$$
\left(v^{v}\right)^{-1} \mathrm{~d} v^{v}=\widetilde{\Xi}^{v}-\left(v^{v}\right)^{-1} \Xi^{v} v^{v}
$$

we thus inductively obtain bounds on $v^{v}$ in $\mathcal{C}^{k+1}\left(\left( \pm\left[T_{n}, T_{n}+1\right]\right) \times Y\right)$. Then, by a compact Sobolev embedding, we find a subsequence $\lim _{\ell \rightarrow \infty} v_{n}(\ell)=\infty$ such that $\left.v^{v_{n}(\ell)}\right|_{\left( \pm\left[T_{n}, T_{n}+1\right]\right) \times Y} \rightarrow v_{n}^{ \pm}$converges in the $\mathcal{C}^{k}$ norm. Again using (82) we see that this convergence is in fact in the $\mathcal{C}^{k+1}$ norm. On these domains we moreover have

$$
\begin{aligned}
\left\|\left(v_{n}^{ \pm}\right)^{*} \Xi-\Xi\right\|_{\mathcal{C}^{k}} & =\lim _{\nu=v_{n}(\ell) \rightarrow \infty}\left\|\left(v^{\nu}\right)^{*} \Xi-\Xi\right\|_{\mathcal{C}^{k}} \\
& \leq \lim _{\nu \rightarrow \infty}\left(\left\|\left(v^{\nu}\right)^{*} \Xi^{\nu}-\Xi\right\|_{\mathcal{C}^{k}}+\left\|\left(v^{\nu}\right)^{-1}\left(\Xi^{\nu}-\Xi\right) v^{\nu}\right\|_{\mathcal{C}^{k}}\right)=0 .
\end{aligned}
$$

First, this implies that $v_{n}^{ \pm} \in \mathcal{G}(Y)$ is independent of $s \in \pm\left[T_{n}, T_{n}+1\right]$. Secondly, by unique continuation (Proposition 8.6), it implies $\left(v_{n}^{ \pm}\right)^{*} \Xi=\Xi$ and hence the limits $v_{n}^{ \pm} \in \mathcal{G}_{A^{ \pm}}$must lie in the stabilizer of the limit connections. Now we can define the gauge transformations $u_{n}^{\ell} \in \mathcal{G}(\mathbb{R} \times Y)$ by $u_{n}^{\ell}=v^{v_{n}(\ell)}\left(v_{n}^{ \pm}\right)^{-1}$ for $\pm s \geq T_{n}+1$, by $u_{n}^{\ell}=\mathbb{1}$ for $|s| \leq T_{n}$, and, for $s \in \pm\left[T_{n}, T_{n}+1\right]$, by an interpolation which satisfies $d\left(u_{n}^{\ell}, \mathbb{1}\right)_{\mathcal{C}^{k+1}\left(\left( \pm\left[T_{n}, T_{n}+1\right]\right) \times Y\right)} \rightarrow 0$ as $\ell \rightarrow \infty$. With this choice we have

$$
\left\|\left(u_{n}^{\ell}\right)^{*} \Xi^{v_{n}(\ell)}-\Xi\right\|_{W_{\delta}^{k, p}\left(\left(\mathbb{R} \backslash\left[-T_{n}-1, T_{n}+1\right]\right) \times Y\right)} \leq \frac{\rho_{n}}{2}
$$

from the exponential decay, as before for $\left(v^{v}\right)^{*} \Xi^{v}$, and

$$
\left\|\left(u_{n}^{\ell}\right)^{*} \Xi^{v_{n}(\ell)}-\Xi\right\|_{\left.W_{\delta}^{k, p}\left(\left[-T_{n}-1, T_{n}+1\right]\right) \times Y\right)} \leq \frac{\rho_{n}}{2}
$$

for all sufficiently large $\ell \geq L_{n}$, from the convergence of $\Xi^{v}$ and $u_{n}^{\ell}$ on compact subsets. Now we can pick $\ell_{n} \geq L_{n}$ so large that $v_{n}:=v_{n}\left(\ell_{n}\right) \rightarrow \infty$ and $\left\|\left(u^{v_{n}}\right)^{*} \Xi^{v_{n}}-\Xi\right\|_{W_{\delta}^{k, p}(\mathbb{R} \times Y)} \leq \rho_{n} \rightarrow 0$. This proves the theorem.

Corollary 7.6 Let $h_{f}$ be a regular perturbation in the sense of Definition 8.2, and let $A^{+}, A^{-} \in \mathcal{A}(Y, \mathcal{L})$ be nondegenerate and irreducible critical points of $\mathcal{C} \mathcal{S}_{\mathcal{L}}+h_{f}$. Then $\mathcal{M}^{1}\left(A^{-}, A^{+} ; X_{f}\right) / \mathbb{R}$ is compact and hence is a finite set.

Proof Assume by contradiction that there is a sequence of distinct points $\left[\Xi^{\nu}\right] \in$ $\mathcal{M}^{1}\left(A^{-}, A^{+} ; X_{f}\right) / \mathbb{R}$. These solutions have index 1 and hence fixed energy by Corollary 6.14 (i). By Theorem 7.2 we can pick a subsequence and representatives $\Xi^{v_{k}}$ 
that converge to a broken trajectory $\left(\Xi_{1}, \ldots, \Xi_{\ell}\right)$ modulo bubbling. By transversality we do not have solutions of negative index, so Corollary 7.4 implies that there is no bubbling, and the index identity in Theorem 7.2 implies that $\ell=1$. Now Theorem 7.5 implies that $\Xi^{v_{k}}$ converges to $\Xi_{1}$ in the $W_{\delta}^{1, p}$-norm. Since $\mathcal{M}^{1}\left(A^{-}, A^{+} ; X_{f}\right) / \mathbb{R}$ is a 0 -manifold this implies that $\Xi^{v_{k}}$ is gauge equivalent to a time-shift of $\Xi_{1}$ in contradiction to the assumption.

Finally we refine the compactness theorem in the case when bubbling is excluded but breaking can take place. The precise convergence statement here will be important for the gluing theory.

Theorem 7.7 Fix a constant $p>1$. Let $f, f^{v}, \Xi^{v}, s_{i}^{v}, u_{i}^{v}$, and $\Xi_{i}$ be as in the conclusion of Theorem 7.2 and suppose that no bubbling occurs, ie the curvature of $\Xi^{v}$ is uniformly bounded, $\left(\left(u_{i}^{v}\right)^{*} \Xi^{v}\right)\left(s_{i}^{v}+\cdot\right)$ converges to $\Xi_{i}$ in the $\mathcal{C}^{k}$ topology on compact sets, and

$$
\sum_{i=1}^{\ell} E_{f}\left(\Xi_{i}\right)=\lim _{\nu \rightarrow \infty} E_{f^{\nu}}\left(\Xi^{v}\right) .
$$

Then the following holds.

(i) If $\mathcal{D}_{\Xi_{i}, \delta}$ is surjective for $i=1, \ldots, \ell$ then so is $\mathcal{D}_{\Xi^{v}, \delta}$ for $v$ sufficiently large.

(ii) If the set of critical points of $\mathcal{C} \mathcal{S}_{\mathcal{L}}+h_{f} v$ is independent of $v$ then, after replacing the broken trajectory $\left(\Xi_{1}, \ldots, \Xi_{\ell}\right)$ by a gauge equivalent one, and for a subsequence, there exists a sequence of gauge transformations $u^{v} \in \mathcal{G}(\mathbb{R} \times Y)$ such that

$$
\begin{aligned}
& \lim _{\nu \rightarrow \infty}\left\|\left(u^{\nu}\right)^{*} \Xi^{\nu}-\Xi_{i}\left(\cdot-s_{i}^{\nu}\right)\right\|_{W^{1, p}\left(I_{i}^{\nu} \times Y\right)}=0, \quad \text { for } i=1, \ldots, \ell, \\
& I_{i}^{v}:= \begin{cases}\left(-\infty, \frac{3}{4} s_{2}^{\nu}+\frac{1}{4} s_{1}^{\nu}\right], & i=1, \\
{\left[\frac{3}{4} s_{i-1}^{v}+\frac{1}{4} s_{i}^{\nu}, \frac{3}{4} s_{i+1}^{\nu}+\frac{1}{4} s_{i}^{\nu}\right],} & i=2, \ldots, \ell-1, \\
{\left[\frac{3}{4} s_{\ell-1}^{v}+\frac{1}{4} s_{\ell}^{v}, \infty\right),} & i=\ell .\end{cases}
\end{aligned}
$$

Proof Fix a constant $\varepsilon>0$ smaller than the constant of Theorem 5.3 and recall that the sequences $s_{i}^{v}$ in Theorem 7.2 are chosen such that

$$
E_{f^{v}}\left(\Xi^{v} ;\left(-\infty, s_{1}^{v}\right]\right)=\varepsilon / 2, \quad E_{f^{v}}\left(\Xi^{v} ;\left[s_{i}^{v}, s_{i+1}^{v}\right]\right)=E_{f}\left(\Xi_{i}\right)
$$

for $v$ sufficiently large and $i=1, \ldots, \ell-1$. Since $s_{i+1}^{v}-s_{i}^{v} \rightarrow \infty$ we have for any $T>0$

$$
E_{f^{\nu}}\left(\Xi^{\nu} ;\left[s_{i}^{v}, s_{i}^{v}+T\right]\right)+E_{f^{\nu}}\left(\Xi^{v} ;\left[s_{i+1}^{v}-T, s_{i+1}^{\nu}\right]\right) \leq E_{f^{\nu}}\left(\Xi^{v} ;\left[s_{i}^{v}, s_{i+1}^{v}\right]\right)
$$


for large $v$. With $v \rightarrow \infty$ this gives $E_{f}\left(\Xi_{i} ;[0, T]\right)+E_{f}\left(\Xi_{i+1} ;[-T, 0]\right) \leq E_{f}\left(\Xi_{i}\right)$ and, by taking the limit $T \rightarrow \infty, E_{f}\left(\Xi_{i+1} ;(-\infty, 0]\right) \leq E_{f}\left(\Xi_{i} ;(-\infty, 0]\right)$. Hence $E_{f}\left(\Xi_{i} ;(-\infty, 0]\right) \leq \varepsilon / 2$ for all $i$. Choose $\tau_{1}, \ldots, \tau_{\ell}$ such that

$$
E_{f}\left(\Xi_{i} ;\left[-\tau_{i}, \tau_{i}\right]\right)=E_{f}\left(\Xi_{i}\right)-\varepsilon / 4
$$

Then $E_{f}\left(\Xi_{i} ;\left[0, \tau_{i}\right]\right) \geq E_{f}\left(\Xi_{i}\right)-3 \varepsilon / 4$ and hence $E_{f^{v}}\left(\Xi^{v} ;\left[s_{i}^{\nu}, s_{i}^{v}+\tau_{i}\right]\right)>E_{f}\left(\Xi_{i}\right)-\varepsilon$ for $v$ sufficiently large. Moreover, $E_{f^{v}}\left(\Xi^{v} ;\left[s_{\ell}^{v}, \infty\right)\right)$ converges to $E_{f}\left(\Xi_{\ell}\right)-\varepsilon / 2$, by (83) and (84). In summary we have for $i=0, \ldots, \ell$ and $v$ sufficiently large

$$
E_{f^{v}}\left(\Xi^{v} ; J_{i}^{v}\right)<\varepsilon, \quad J_{i}^{v}:= \begin{cases}\left(-\infty, s_{1}^{v}\right], & i=0, \\ {\left[s_{i}^{v}+\tau_{i}, s_{i+1}^{v}\right],} & i=1, \ldots, \ell-1, \\ {\left[s_{\ell}^{v}+\tau_{\ell}, \infty\right),} & i=\ell .\end{cases}
$$

Now choose gauge transformations $v_{i}^{v}$ on $J_{i}^{v} \times Y$ such that $\left(v_{i}^{\nu}\right)^{*} \Xi^{v}$ is in temporal gauge on $J_{i}^{v} \times Y$. Thus each connection $\left(v_{i}^{v}\right)^{*} \Xi^{v}$ is represented by a smooth path $\widetilde{A}_{i}^{v}: J_{i} \rightarrow \mathcal{A}(Y, \mathcal{L})$. Then it follows from Theorem 5.3 that there are critical points $B_{i}^{v} \in \operatorname{Crit}\left(\mathcal{C S}_{\mathcal{L}}+h_{f^{v}}\right)$ and positive constants $C_{0}$ and $\delta_{0}$ such that, for $i=0, \ldots, \ell$, $\tau \geq \tau_{i}+1$, and $v$ sufficiently large, we have

$$
\left\|\widetilde{A}_{i}^{v}-B_{i}^{v}\right\|_{\mathcal{C}^{0}\left(J_{i}^{\nu}(\tau) \times Y\right)}+\left\|\widetilde{A}_{i}^{v}-B_{i}^{v}\right\|_{W^{1, p}\left(J_{i}^{\nu}(\tau) \times Y\right), B_{i}^{\nu}} \leq C_{0} e^{-\delta_{0}\left(\tau-\tau_{i}\right)} \sqrt{\varepsilon}
$$

Here we abbreviate $\tau_{0}:=0$ and

$$
J_{i}^{v}(\tau):= \begin{cases}\left(-\infty, s_{1}^{v}-\tau\right], & i=0, \\ {\left[s_{i}^{v}+\tau, s_{i+1}^{v}-\tau\right],} & i=1, \ldots, \ell-1, \\ {\left[s_{\ell}^{v}+\tau, \infty\right),} & i=\ell .\end{cases}
$$

Moreover we use the fact that the constants in Theorem 5.3 can be chosen uniform for all $f^{v}$. Since the estimate is gauge invariant we may modify the gauge transformations $v_{i}^{v}$ so that the sequence $B_{i}^{v}$ converges in the $\mathcal{C}^{k}$-norm to the critical point $B_{i}$ in the assertion of Theorem 7.2 for every $i$ (see Proposition 3.7). Then (86) continues to hold if we drop the subscript $B_{i}^{v}$ in the $W^{1, p}$-norm and replace $C_{0}$ with a possibly larger constant, still denoted by $C_{0}$.

Under the assumption of (ii) we may choose $v_{i}^{v}$ so that $B_{i}^{v}=B_{i}$ is independent of $v$. Now we can argue as in the proof of Theorem 7.5. Combining (86) with $B_{i}^{v}=B_{i}$ and 
the exponential decay of $\Xi_{i}$ and $\Xi_{i+1}$ we obtain the estimates

$$
\begin{aligned}
\left\|\left(v_{0}^{\nu}\right)^{*} \Xi^{v}-\Xi_{1}\left(\cdot-s_{1}^{\nu}\right)\right\|_{W^{1, p}\left(\left(-\infty, s_{1}^{\nu}-\tau\right] \times Y\right)} & \leq C_{1} e^{-\delta_{0} \tau}, \\
\left\|\left(v_{i}^{\nu}\right)^{*} \Xi^{v}-\Xi_{i}\left(\cdot-s_{i}^{\nu}\right)\right\|_{W^{1, p}\left(\left[s_{i}^{\nu}+\tau, \frac{3}{4} s_{i+1}^{\nu}+\frac{1}{4} s_{i}^{\nu}\right] \times Y\right)} & \leq C_{1} e^{-\delta_{0}\left(\tau-\tau_{i}\right)}, \\
\left\|\left(v_{i}^{\nu}\right)^{*} \Xi^{v}-\Xi_{i+1}\left(\cdot-s_{i+1}^{\nu}\right)\right\|_{W^{1, p}\left(\left[\frac{3}{4} s_{i}^{\nu}+\frac{1}{4} s_{i+1}^{\nu}, s_{i+1}^{\nu}-\tau\right] \times Y\right)} & \leq C_{1} e^{-\delta_{0}\left(\tau-\tau_{i}\right)}, \\
\left\|\left(v_{\ell}^{\nu}\right)^{*} \Xi^{v}-\Xi_{\ell}\left(\cdot-s_{\ell}^{\nu}\right)\right\|_{W^{1, p}\left(\left[s_{\ell}^{v}+\tau, \infty\right) \times Y\right)} & \leq C_{1} e^{-\delta_{0}\left(\tau-\tau_{\ell}\right)}
\end{aligned}
$$

for $v$ sufficiently large, some constant $C_{1}$, and $i=1, \ldots, \ell-1$. Fix a constant $\rho>0$ and choose $\tau$ so large that

$$
C_{1} e^{-\delta_{0}\left(\tau-\tau_{i}\right)} \leq \rho / 4 \quad \text { for } i=1, \ldots, \ell .
$$

Then, on the interval $\left[s_{i}^{v}-\tau-1, s_{i}^{v}-\tau\right] \subset J_{i-1}^{v}(\tau)$ the connections $\left(v_{i-1}^{v}\right)^{*} \Xi^{v}$ and $\left(u_{i}^{\nu}\right)^{*} \Xi^{\nu}$ are both $W^{1, p}$ close to $\Xi_{i}\left(\cdot-s_{i}^{\nu}\right)$ Thus $\left(\left(v_{i-1}^{\nu}\right)^{-1} u_{i}^{\nu}\right)\left(\cdot+s_{i}^{\nu}\right)$ is bounded in $W^{2, p}([-\tau-1,-\tau] \times Y)$ and thus, for a subsequence, converges to a gauge transformation $g_{i}^{-} \in \mathcal{G}^{2, p}([-\tau-1,-\tau] \times Y)$. For the limit we obtain $\left(g_{i}^{-}\right)^{*} \Xi_{i}=\Xi_{i}$ on $(-\infty,-1]$ as in Theorem 7.5, and we deduce that $g_{i}^{-} \in \mathcal{G}_{B_{i}}$. Similarly, we can pick the subsequence such that $\left(\left(v_{i}^{\nu}\right)^{-1} u_{i}^{v}\right)\left(\cdot+s_{i}^{\nu}\right) \rightarrow g_{i}^{+} \in \mathcal{G}_{B_{i+1}}$ in $W^{2, p}([\tau, \tau+1] \times Y)$ with $\left(g_{i}^{+}\right)^{*} \Xi_{i}=\Xi_{i}$ on $[1, \infty)$. With this we can now construct a sequence $u^{v} \in \mathcal{G}(\mathbb{R} \times Y)$ that satisfies

- $u^{v}(s)=v_{0}^{v}(s) g_{1}^{-}$for $s \in\left(-\infty, s_{1}^{v}-\tau-1\right]$,

- $u^{v}(s)=u_{1}^{v}(s)$ for $s \in\left[s_{1}^{v}-\tau, s_{1}^{v}+\tau\right]$,

- $u^{v}(s)=v_{1}^{v}(s) g_{i}^{+}$for $s \in\left[s_{1}^{v}+\tau+1, s_{2}^{v}-\tau-1\right]$,

- $u^{\nu}(s)=u_{i}^{\nu}(s)\left(g_{i}^{-}\right)^{-1} g_{i-1}^{+}\left(g_{i-1}^{-}\right)^{-1} \ldots\left(g_{2}^{-}\right)^{-1} g_{1}^{+}$for $s \in\left[s_{i}^{\nu}-\tau, s_{i}^{\nu}+\tau\right]$ and $i=2, \ldots, \ell$,

- $u^{v}(s)=v_{i}^{v}(s) g_{i}^{+}\left(g_{i}^{-}\right)^{-1} g_{i-1}^{+} \ldots\left(g_{2}^{-}\right)^{-1} g_{1}^{+}$for $s \in\left[s_{i}^{v}+\tau+1, s_{i+1}^{v}-\tau-1\right]$ and $i=2, \ldots, \ell$,

- $\left(\left(u_{i}^{\nu}\right)^{-1} u^{v}\right)\left(\cdot+s_{i}^{\nu}\right) \rightarrow\left(g_{i}^{-}\right)^{-1} g_{i-1}^{+}\left(g_{i-1}^{-}\right)^{-1} \ldots\left(g_{2}^{-}\right)^{-1} g_{1}^{+}$as $v \rightarrow \infty$ in $W^{2, p}([-\tau-1, \tau+1] \times Y, \mathrm{G})$ for $i=1, \ldots, \ell$,

- $\operatorname{dist}_{W^{2, p}\left(\left[s_{i}^{v}+\tau, s_{i+1}^{v}-\tau\right]\right)}\left(\left(v_{i}^{v}\right)^{-1} u^{v}, g_{i}^{+}\left(g_{i}^{-}\right)^{-1} g_{i-1}^{+}\left(g_{i-1}^{-}\right)^{-1} \ldots\left(g_{2}^{-}\right)^{-1} g_{1}^{+}\right) \rightarrow 0$ as $v \rightarrow \infty$ for $i \stackrel{=}{=} 0, \ldots, \ell$.

At the same time we replace the broken trajectory $\left(\Xi_{1}, \ldots, \Xi_{\ell}\right)$ with $\Xi_{1}^{\prime}:=\Xi_{1}$ and $\Xi_{i}^{\prime}:=\left(\left(g_{i}^{-}\right)^{-1} g_{i-1}^{+}\left(g_{i-1}^{-}\right)^{-1} \ldots\left(g_{2}^{-}\right)^{-1} g_{1}^{+}\right)^{*} \Xi_{i}$ for $i=2, \ldots, \ell$. Note that this again defines a broken trajectory $\left(\Xi_{1}^{\prime}, \ldots, \Xi_{\ell}^{\prime}\right)$ between the critical points

$$
\begin{aligned}
\lim _{s \rightarrow \infty} \Xi_{i}^{\prime} & =\left(\left(g_{i}^{-}\right)^{-1} g_{i-1}^{+} \ldots\left(g_{2}^{-}\right)^{-1} g_{1}^{+}\right)^{*} B_{i+1} \\
& =\left(\left(g_{i}^{-}\right)^{-1} g_{i-1}^{+} \ldots\left(g_{2}^{-}\right)^{-1} g_{1}^{+}\right)^{*}\left(\left(g_{i+1}^{-}\right)^{-1} g_{i}^{+}\right)^{*} B_{i+1}=\lim _{s \rightarrow-\infty} \Xi_{i+1}^{\prime} .
\end{aligned}
$$


Here we used the fact that $g_{i+1}^{-}, g_{i}^{+} \in \mathcal{G}_{B_{i+1}}$. The convergence of $\left(u_{i}^{v}\right)^{*} \Xi^{v}$ then implies

$$
\left\|\left(u^{\nu}\right)^{*} \Xi^{v}-\Xi_{i}^{\prime}\left(\cdot-s_{i}^{\nu}\right)\right\|_{W^{1, p}\left(\left[s_{i}^{\nu}-\tau, s_{i}^{\nu}+\tau\right] \times Y\right)} \leq \rho / 3
$$

for large $v$ and $i=1, \ldots, \ell$, and from the exponential decay (87) we obtain

$$
\begin{gathered}
\left\|\left(u^{v}\right)^{*} \Xi^{v}-\Xi_{i}^{\prime}\left(\cdot-s_{i}^{v}\right)\right\|_{W^{1, p}\left(\left[s_{i}^{v}+\tau, \frac{3}{4} s_{i+1}^{v}+\frac{1}{4} s_{i}^{v}\right] \times Y\right)} \leq \rho / 3, \\
\left\|\left(u^{v}\right)^{*} \Xi^{v}-\Xi_{i}^{\prime}\left(\cdot-s_{i}^{v}\right)\right\|_{W^{1, p}\left(\left[\frac{3}{4} s_{i-1}^{v}+\frac{1}{4} s_{i}^{\nu}, s_{i}^{v}-\tau\right] \times Y\right)} \leq \rho / 3,
\end{gathered}
$$

for large $v$, large $\tau$, and $i=1, \ldots, \ell$. Here we denote $s_{0}^{v}:=-\infty$ and $s_{\ell+1}^{v}:=\infty$, and we use the fact that $\left(g_{i}^{+}\right)^{*} \Xi_{i}=\Xi_{i}$ on $[1, \infty)$ and $\left(g_{i}^{-}\right)^{*} \Xi_{i}=\Xi_{i}$ on $(-\infty,-1]$. Thus, for every $\rho>0$, we have a subsequence $\left(v_{n}\right)_{n \in \mathbb{N}}$ and a sequence of gauge transformations $u_{\rho}^{v_{n}}$ such that

$$
\left\|\left(u_{\rho}^{v_{n}}\right)^{*} \Xi^{v_{n}}-\Xi_{i}^{\prime}\left(\cdot-s_{i}^{v_{n}}\right)\right\|_{W^{1, p}\left(I_{i}^{v_{n}} \times Y\right)} \leq \rho
$$

holds for all sufficiently large $n \geq N_{\rho}$. Assertion (ii) then follows by taking a diagonal subsequence.

To prove (i) we can assume by contradiction that, after passing to a subsequence, none of the $\mathcal{D}_{\Xi^{v}, \delta}$ is surjective. Then we use the $\mathcal{C}^{0}$-estimate of (86) and the same patching construction as for (ii) to find a further subsequence and a sequence of gauge transformations $u^{v} \in \mathcal{G}(\mathbb{R} \times Y)$ such that

$$
\lim _{\nu \rightarrow \infty}\left\|\left(u^{\nu}\right)^{*} \Xi^{\nu}-\Xi_{i}^{\prime}\left(\cdot-s_{i}^{\nu}\right)\right\|_{\mathcal{C}^{0}\left(I_{i}^{\nu} \times Y\right)}=0
$$

for $i=1, \ldots, \ell$. (The $\mathcal{C}^{0}$-estimate holds on increasingly large domains because $B_{i}^{v} \rightarrow B_{i}$ converges in $\mathcal{C}^{0}(\mathbb{R} \times Y)$ - but not in $W^{1, p}(\mathbb{R} \times Y)$.) By Theorem 6.9 (iv) the surjectivity of the linearized operators $\mathcal{D}_{\Xi^{v}, \delta}$ is independent of a timeshift in the weight function, or equivalently in the connection. Hence, applying an overall timeshift to each element of the sequence $\Xi^{v}$, we may assume w.l.o.g. that $s_{1}^{v}=0$ and for each $i \geq 2$ we have $s_{i}^{v} \rightarrow \infty$. By assumption, the linearized operator $w \mathcal{D}_{\Xi_{i}, \delta} w^{-1}=\nabla_{s}+\mathcal{H}_{A_{i}}-I_{\lambda}$ is surjective on the unweighted Sobolev spaces, see Remark 6.10 (iv), and so are the operators $\nabla_{s}+\mathcal{H}_{A_{i}}-I_{\delta}$. (Recall that $\lambda=\partial_{s} V=w^{-1} \partial_{s} w$ denotes the derivative of the weight function.) Equivalently, the adjoint operators $-\nabla_{s}+\mathcal{H}_{A_{i}}-I_{\lambda}$ resp. $-\nabla_{s}+\mathcal{H}_{A_{i}}-I_{\delta}$ are injective. Hence there is a constant $c$ such that

$$
\|\xi\|_{L^{p}} \leq c\left\|-\nabla_{s} \xi+\mathcal{H}_{A_{i}} \xi-I_{\lambda} \xi\right\|_{L^{p}}, \quad\|\xi\|_{L^{p}} \leq c\left\|-\nabla_{s} \xi+\mathcal{H}_{A_{i}} \xi-I_{\delta} \xi\right\|_{L^{p}}
$$

for every $\xi \in W^{1, p}\left(\mathbb{R} \times Y, \mathrm{~T}^{*} Y \otimes \mathfrak{g} \oplus \mathfrak{g}\right)$. This estimate is stable under $\mathcal{C}^{0}$-small perturbations of $\Xi_{i}$ and under the action of the gauge group. Hence, enlarging the 
constant $c$ if necessary, we obtain

$$
\operatorname{supp} \xi \subset I_{i}^{v} \times Y \quad \Longrightarrow \quad\|\xi\|_{L^{p}} \leq c\left\|-\nabla_{s} \xi+\mathcal{H}_{A^{\nu}} \xi-I_{\lambda} \xi\right\|_{L^{p}}
$$

for all $\xi$ and $i$ and for $v$ sufficiently large. For $i \geq 1$ this follows directly from (88) with $s_{1}^{v}=0$. For $i \geq 2$ we use the fact that $\lambda(s)=\delta$ for all $s \in I_{i}^{v}$, so we can estimate $\left\|-\nabla_{S} \xi+\mathcal{H}_{A^{v}} \xi-I_{\lambda} \xi\right\|_{L^{p}}$ by

$$
\left\|-\nabla_{s} \xi+\mathcal{H}_{A_{i}\left(\cdot-s_{i}^{v}\right)} \xi-I_{\delta} \xi\right\|_{L^{p}}-\left\|\left(u^{v}\right)^{*} \Xi^{v}-\Xi_{i}\left(\cdot-s_{i}^{v}\right)\right\|_{\mathcal{C}^{0}\left(I_{i}^{v} \times Y\right)}\|\xi\|_{L^{p}}
$$

and identify the first term of this with

$$
\left\|\left(-\nabla_{s}+\mathcal{H}_{A_{i}}-I_{\delta}\right) \xi\left(\cdot+s_{i}^{\nu}\right)\right\|_{L^{p}} \geq c^{-1}\|\xi\|_{L^{p}} .
$$

Now for each $v$ we can choose a partition of unity $h_{i}^{\nu}: \mathbb{R} \rightarrow[0,1]$ with $\operatorname{supp} h_{i}^{\nu} \subset I_{i}^{\nu}$ and $\sum_{i}\left\|\partial_{s} h_{i}^{v}\right\|_{L^{\infty}} \rightarrow 0$. Then we obtain

$$
\begin{aligned}
\|\xi\|_{L^{p}} \leq \sum_{i=1}^{\ell}\left\|h_{i}^{\nu} \xi\right\|_{L^{p}} & \leq c \sum_{i=1}^{\ell}\left\|h_{i}^{v}\left(-\nabla_{s} \xi+\mathcal{H}_{A^{v}} \xi-I_{\lambda} \xi\right)-\left(\partial_{S} h_{i}^{\nu}\right) \xi\right\|_{L^{p}} \\
& \leq \ell c\left\|-\nabla_{S} \xi+\mathcal{H}_{A^{v}} \xi-I_{\lambda} \xi\right\|_{L^{p}}+\sum_{i=1}^{\ell}\left\|\partial_{S} h_{i}^{v}\right\|_{L^{\infty}}\|\xi\|_{L^{p}} .
\end{aligned}
$$

This shows that the operator $-\nabla_{s}+\mathcal{H}_{A^{v}}-I_{\lambda}$ is injective on the unweighted Sobolev spaces for $v$ sufficiently large, and hence its adjoint $\nabla_{S}+\mathcal{H}_{A^{v}}-I_{\lambda}$ is surjective. Since the latter operator is conjugate to $\mathcal{D}_{\Xi^{\nu}, \delta}$ this is a contradiction to the assumption, and the theorem is proved.

\section{Transversality}

Let $(Y, g)$ be a compact oriented Riemannian 3-manifold with metric $g$ and boundary $\partial Y=\Sigma$, and let $\mathcal{L} \subset \mathcal{A}(\Sigma)$ be a gauge invariant, monotone, irreducible Lagrangian submanifold satisfying (L1)-(L3). Then $\mathbb{R} \times Y$ naturally is a Riemannian 4-manifold with boundary space-time splitting and tubular ends in the sense of Definition 6.2. In order to complete the instanton data we must also choose a perturbation. A detailed construction of holonomy perturbations is given in Appendix D. In this section we concentrate on achieving transversality by the choice of perturbation.

Fix an embedding $\beta:[-1,1] \times \mathbb{D} \rightarrow \operatorname{int}(Y)$ and denote by $\Gamma_{m}$ the set of finite sequences $\gamma=\left(\gamma_{1}, \ldots, \gamma_{m}\right)$ of embeddings $\gamma_{i}: S^{1} \times \mathbb{D} \rightarrow \operatorname{int}(Y)$ that agree with $\beta$ in a neighbourhood of $\{0\} \times \mathbb{D}$. Every $\gamma \in \Gamma_{m}$ gives rise to a map

$$
\rho=\left(\rho_{1}, \ldots, \rho_{m}\right): \mathbb{D} \times \mathcal{A}(Y) \rightarrow \mathrm{G}^{m}
$$


where $\rho_{i}(z, A)$ is the holonomy of the connection $A$ around the loop $\gamma_{i}(\cdot, z)$. Let $\mathcal{F}_{m}:=\mathcal{C}_{0}^{\infty}\left(\mathbb{D} \times \mathrm{G}^{m}\right)^{\mathrm{G}}$ denote the space of conjugation invariant real valued compactly supported smooth functions on $\mathbb{D} \times \mathrm{G}^{m}$. Each pair $(\gamma, f) \in \Gamma_{m} \times \mathcal{F}_{m}$ determines a smooth function $h_{f}: \mathcal{A}(Y) \rightarrow \mathbb{R}$ via

$$
h_{f}(A):=\int_{\mathbb{D}} f(z, \rho(z, A)) \mathrm{d}^{2} z .
$$

The differential $\mathrm{d} h_{f}(A): \mathrm{T}_{A} \mathcal{A}(Y) \rightarrow \mathbb{R}$ has the form

$$
\mathrm{d} h_{f}(A) \alpha=\int_{Y}\left\langle X_{f}(A) \wedge \alpha\right\rangle .
$$

Here $X_{f}: \mathcal{A}(Y) \rightarrow \Omega^{2}(Y, \mathfrak{g})$ is a smooth function satisfying (10). We emphasize that the tuple $(11, \ldots, \mathbb{1})$ is a critical point of every conjugation invariant function $\mathrm{G}^{m} \rightarrow \mathbb{R}$ and hence the trivial connection $A=0$ is always a critical point of the perturbed Chern-Simons functional $\mathcal{C S}_{\mathcal{L}}+h_{f}$; it is nondegenerate by assumption (L3).

Definition 8.1 Fix a perturbation $(\gamma, f) \in \Gamma_{m} \times \mathcal{F}_{m}$ and two nondegenerate critical points $A^{ \pm} \in \operatorname{Crit}\left(\mathcal{C S}_{\mathcal{L}}+h_{f}\right)$. A finite energy solution $A: \mathbb{R} \rightarrow \mathcal{A}(Y, \mathcal{L})$ of the boundary value problem (14) with limits $\lim _{s \rightarrow \pm \infty} A(s)=A^{ \pm}$is called regular if the operator $\mathcal{D}_{\mathbb{A}, \delta}$ defined in (71) is surjective for every sufficiently small constant $\delta>0$. (This condition is independent of $k$ and $p$.)

Definition 8.2 A pair $(\gamma, f) \in \Gamma_{m} \times \mathcal{F}_{m}$ is called regular (for $(Y, g)$ and $\mathcal{L}$ ) if it satisfies the following.

(i) Every nontrivial critical point of the perturbed Chern-Simons functional $\mathcal{C S}_{\mathcal{L}}+h_{f}$ is irreducible and nondegenerate, ie if $A \in \mathcal{A}(Y, \mathcal{L})$ is not gauge equivalent to the trivial connection and satisfies $F_{A}+X_{f}(A)=0$ then $H_{A}^{0}=0$ and $H_{A, f}^{1}=0$.

(ii) Let $A: \mathbb{R} \rightarrow \mathcal{A}(Y, \mathcal{L})$ be a finite energy solution of the boundary value problem (14) with $\delta_{f}(\mathbb{A}) \leq 7$ and suppose that at most one of the limits $A^{ \pm}$is gauge equivalent to the trivial connection. Then the operator $\mathcal{D}_{\mathbb{A}, \delta}$ defined in (71) is surjective for every integer $k \geq 1$, every $p>1$, and every sufficiently small constant $\delta>0$.

For every $\gamma \in \Gamma_{m}$ the set of regular elements $f \in \mathcal{F}_{m}$ will be denoted by $\mathcal{F}_{\text {reg }}(\gamma)$.

If $f \in \mathcal{F}_{\text {reg }}(\gamma)$ and $\left(\left[A^{-}\right],\left[A^{+}\right]\right) \neq(0,0)$ then it follows from the discussion in Section 6 that the moduli space $\mathcal{M}\left(A^{-}, A^{+} ; X_{f}\right)$, introduced in (62) and the beginning of Section 7 , is a smooth manifold of local dimension

$$
\operatorname{dim}_{[\mathbb{A}]} \mathcal{M}\left(A^{-}, A^{+} ; X_{f}\right)=\delta_{f}(\mathbb{A}) .
$$


For every integer $k \geq 1$ we introduce the following seminorm on the space of perturbations

$$
\left|\left\|X_{f} \mid\right\|_{k}:=\sup _{A \in \mathcal{A}(Y, \mathcal{L})}\left(\frac{\left\|X_{f}(A)\right\|_{\mathcal{C}^{k}}}{\left(1+\|A\|_{C^{k}}\right)^{k}}+\sup _{\alpha \in \mathrm{T}_{A} \mathcal{A}(Y, \mathcal{L})} \frac{\left\|\mathrm{d} X_{f}(A) \alpha\right\|_{\mathcal{C}^{k-1}}}{\|\alpha\|_{\mathcal{C}^{k-1}}\left(1+\|A\|_{\mathcal{C}^{k-1}}\right)^{k-1}}\right) .\right.
$$

We will apply this notation to the difference $X_{f}-X_{f^{\prime}}$ associated to two pairs $(\gamma, f) \in$ $\Gamma_{m} \times \mathcal{F}_{m}$ and $\left(\gamma^{\prime}, f^{\prime}\right) \in \Gamma_{m^{\prime}} \times \mathcal{F}_{m^{\prime}}$. This difference can be written as $X_{f-f^{\prime}}$ associated to the union $\gamma \cup \gamma^{\prime}:=\left(\gamma_{1}, \ldots, \gamma_{m}, \gamma_{1}^{\prime}, \ldots, \gamma_{m^{\prime}}\right) \in \Gamma_{m+m^{\prime}}$, where $f$ and $f^{\prime}$ are extended to elements of $\mathcal{F}_{m+m^{\prime}}$ in the obvious way. Then Proposition D.1 implies that $\left\|X_{f_{v}}-X_{f_{0}} \mid\right\|_{k} \rightarrow 0$ for $\left\|f_{v}-f_{0}\right\|_{\mathcal{C}^{k+1}} \rightarrow 0$.

Theorem 8.3 (i) For every $\gamma \in \Gamma_{m}$ the set of all $f \in \mathcal{F}_{m}$ that satisfy condition (i) in Definition 8.2 is open in $\mathcal{F}_{m}$ with respect to the $\mathcal{C}^{2}$-topology.

(ii) Let $\left(\gamma_{0}, f_{0}\right) \in \Gamma_{m_{0}} \times \mathcal{F}_{m_{0}}$ be such that every nontrivial critical point of $\mathcal{C} \mathcal{S}_{\mathcal{L}}+h_{f_{0}}$ is irreducible. Then, for every $\varepsilon>0$ and every $k \in \mathbb{N}$, there exists an $n \in \mathbb{N}$ and a pair $(\gamma, f) \in \Gamma_{n} \times \mathcal{F}_{n}$ that satisfies condition (i) in Definition 8.2 and $\left|\left\|X_{f}-X_{f_{0}} \mid\right\|_{k}<\varepsilon\right.$.

The zero perturbation satisfies the assumptions of Theorem 8.3 (ii) by (L3). Transversality for the critical points near the unperturbed equation was established by Taubes [30]. The extension to large perturbations requires another proof, similar to that of the following transversality result for trajectories.

Theorem 8.4 (i) The set $\mathcal{F}_{\text {reg }}(\gamma)$ is open in $\mathcal{F}_{m}$ with respect to the $C^{2}$-topology for every $m \in \mathbb{N}$ and every $\gamma \in \Gamma_{m}$.

(ii) Assume that $\left(\gamma_{0}, f_{0}\right) \in \Gamma_{m_{0}} \times \mathcal{F}_{m_{0}}$ satisfies condition (i) in Definition 8.2. Then, for every $\varepsilon>0$ and $k \in \mathbb{N}$, there exists an $n \in \mathbb{N}$ and another pair $(\gamma, f) \in \Gamma_{n} \times \mathcal{F}_{n}$ that is regular, ie $f \in \mathcal{F}_{\text {reg }}(\gamma)$, and satisfies

$$
\begin{gathered}
\operatorname{Crit}\left(\mathcal{C S}_{\mathcal{L}}+h_{f_{0}}\right)=\operatorname{Crit}\left(\mathcal{C} \mathcal{S}_{\mathcal{L}}+h_{f}\right), \\
A \in \operatorname{Crit}\left(\mathcal{C} \mathcal{S}_{\mathcal{L}}+h_{f_{0}}\right) \Longrightarrow h_{f}(A)=h_{f_{0}}(A), \\
\left|\left\|X_{f}-X_{f_{0}} \mid\right\|_{k}<\varepsilon\right.
\end{gathered}
$$

Note that we do not construct a Banach space of perturbations in which regular ones are of Baire second category. The main reason for this is that the loops in the interior of $Y$ do not form a Banach space.

Remark 8.5 Fix a point $y_{0} \in \operatorname{int}(Y)$. For every based, embedded loop $\gamma:[0,1] \rightarrow$ $\operatorname{int}(Y)$ with $\gamma(0)=\gamma(1)=y_{0}$ denote by $\rho_{\gamma}: \mathcal{A}(Y) \rightarrow \mathrm{G}$ the holonomy map. For 
later reference we state two facts that follow from the equivalence between connection 1 -forms and parallel transport. (Note that it suffices to use embedded loops in the interior.)

(i) Two connections $A, B \in \mathcal{A}(Y)$ are gauge equivalent if and only if there is a $g_{0} \in \mathrm{G}$ such that

$$
\rho_{\gamma}(B)=g_{0}^{-1} \rho_{\gamma}(A) g_{0}
$$

for every based embedded loop $\gamma$.

(ii) Let $A \in \mathcal{A}(Y)$ and $\alpha \in \Omega^{1}(Y, \mathfrak{g})$. Then $\alpha \in \operatorname{imd}_{A}$ if and only if there is a $\xi_{0} \in \mathfrak{g}$ such that

$$
\mathrm{d} \rho_{\gamma}(A) \alpha=\rho_{\gamma}(A) \xi_{0}-\xi_{0} \rho_{\gamma}(A)
$$

for every based embedded loop $\gamma$.

Proof of Theorem 8.3 Assertion (i) follows from the fact that the conditions $H_{A}^{0}=0$ and $H_{A, f}^{1}=0$ are open with respect to $\mathcal{C}^{2}$-variations of $f$ and $A$. The conditions are moreover gauge invariant, and the set of nontrivial critical points of $\mathcal{C} \mathcal{S}_{\mathcal{L}}+h_{f}$ is compact in $\mathcal{A}(Y, \mathcal{L}) / \mathcal{G}(Y)$ for every perturbation $f$. (This follows from Uhlenbeck compactness $[32 ; 34]$ since $F_{A}=-X_{f}(A)$ is $L^{\infty}$-bounded.) The proof of (ii) has three steps.

Step 1 Let $\left(\gamma_{0}, f_{0}\right) \in \Gamma_{m_{0}} \times \mathcal{F}_{m_{0}}$ be given. Then there is a $\gamma \in \Gamma_{m}$ with $\gamma_{i}=\gamma_{0 i}$ for $i=1, \ldots, m_{0}$ satisfying the following condition. Define $\sigma: \mathcal{A}(Y) \rightarrow \mathrm{G}^{m}$ by

$$
\sigma(A):=\rho(0, A)=\left(\rho_{1}(0, A), \ldots, \rho_{m}(0, A)\right) .
$$

Then, for every critical point $A \in \operatorname{Crit}\left(\mathcal{C S}_{\mathcal{L}}+h_{f_{0}}\right)$ and every nonzero 1 -form $\eta \in$ $\Omega^{1}(Y, \mathfrak{g})$ satisfying

$$
\mathrm{d}_{A} \eta+\mathrm{d} X_{f_{0}}(A) \eta=0, \quad \mathrm{~d}_{A}^{*} \eta=0,\left.\quad \eta\right|_{\partial Y} \in \mathrm{T}_{A} \mathcal{L},\left.\quad * \eta\right|_{\partial Y}=0,
$$

the vector $[\mathrm{d} \sigma(A) \eta] \in \mathrm{T}\left(\mathrm{G}^{m} / \mathrm{G}\right)$ is nonzero.

The trivial connection is nondegenerate by assumption (L3), so for $\eta \neq 0$ we must have $[A] \neq[0]$, and so by assumption $A$ is irreducible. The condition $[\mathrm{d} \sigma(A) \eta] \neq 0$ is open with respect to variations of $(A, \eta)$, and it is invariant under gauge transformations $(A, \eta) \mapsto\left(u^{*} A, u^{-1} \eta u\right)$. Moreover, the set of gauge equivalence classes of pairs $(A, \eta) \in \operatorname{Crit}\left(\mathcal{C S}_{\mathcal{L}}+h_{f_{0}}\right) \times \Omega^{1}(Y, \mathfrak{g})$ that satisfy $\|\eta\|_{L^{2}}=1,[A] \neq[0]$, and (89) is compact. (For $\eta$ this follows from elliptic estimates for the operator $\mathrm{d}_{A} \oplus \mathrm{d}_{A}^{*}$ with boundary condition $\left.* \eta\right|_{\partial Y}=0$; see eg [34, Theorem D].) Hence it suffices to construct $\gamma$ for a single such pair $(A, \eta)$. We shall use Remark 8.5 (ii) to construct $\gamma$. In each 
step it suffices to find the loops $\theta \mapsto \gamma_{i}(\theta, 0)$ (with base point $y_{0}:=\beta(0,0)$ ). Since the condition is open with respect to smooth variations of $\gamma$, these loops can be deformed and extended to the required embeddings of $\mathbb{D} \times S^{1}$ into the interior of $Y$.

Since $A$ is irreducible we can choose the loops $\gamma_{m_{0}+1}$ and $\gamma_{m_{0}+2}$ such that the matrices $g_{1}:=\rho_{m_{0}+1}(0, A)$ and $g_{2}:=\rho_{m_{0}+2}(0, A)$ do not commute. Then $\sigma(A)$ lies in the free part of $\mathrm{G}^{m}$. The tangent space of the $\mathrm{G}$-orbit through $\sigma(A)$ is

$$
V_{0}:=\left\{v=\left(\sigma_{i}(A) \xi-\xi \sigma_{i}(A)\right)_{i=1, \ldots, m} \mid \xi \in \mathfrak{g}\right\} \subset \mathrm{T}_{\sigma(A)} \mathrm{G}^{m} .
$$

We prove that $\gamma$ can be chosen such that $\mathrm{d} \sigma(A) \eta \notin V_{0}$.

Since $\eta \perp \mathrm{im} \mathrm{d}_{A}$, it follows from Remark 8.5 (ii) that for every $\xi \in \mathfrak{g}$ there is a based loop $\gamma$ such that

$$
\mathrm{d} \rho_{\gamma}(A) \eta \neq \rho_{\gamma}(A) \xi-\xi \rho_{\gamma}(A) .
$$

Since the map $\xi \mapsto\left(g_{1} \xi-\xi g_{1}, g_{2} \xi-\xi g_{2}\right)$ is injective there is a constant $C$ such that for $|\xi| \geq C$ condition (90) holds for one of the loops $\gamma_{m+1}(0, \cdot)$ or $\gamma_{m+2}(0, \cdot)$. The compact set $\{|\xi| \leq C\}$ can be covered by finitely many open sets $U_{j}$, on each of which condition (90) holds with the same loop $\gamma_{m+2+j}$. Thus we have proved that for every $\xi \in \mathfrak{g}$ there exists an $i$ such that (90) holds with $\gamma=\gamma_{i}$. This implies that $\mathrm{d} \sigma(A) \eta$ is not contained in $V_{0}$ and hence does not vanish in the tangent space of the quotient $\mathrm{G}^{m} / \mathrm{G}$.

Step 2 Let $\gamma \in \Gamma_{m}$ be as in Step 1 and fix $p>3$. For $k \in \mathbb{N}$ and $\varepsilon>0$ denote

$$
\mathcal{F}_{m}^{k, \varepsilon}:=\left\{f \in \mathcal{C}^{k+1}\left(\mathbb{D} \times \mathrm{G}^{m}\right)^{\mathrm{G}} \mid\left\|f-f_{0}\right\|_{\mathcal{C}^{k+1}}<\varepsilon\right\},
$$

let $\mathcal{A}^{1, p}(Y, \mathcal{L})$ and $\mathcal{G}^{2, p}(Y)$ denote the $W^{1, p}$ - and $W^{2, p}$-closure of $\mathcal{A}(Y, \mathcal{L})$ and $\mathcal{G}(Y)$ respectively and denote

$$
\widetilde{\mathcal{M}}^{*}\left(\mathcal{F}_{m}^{k, \varepsilon}\right):=\left\{(A, f) \in \mathcal{A}^{1, p}(Y, \mathcal{L}) \times \mathcal{F}_{m}^{k, \varepsilon} \mid F_{A}+X_{f}(A)=0,[A] \neq[0]\right\} .
$$

Then for every $k \in \mathbb{N}$ there is an $\varepsilon>0$ such that the moduli space

$$
\mathcal{M}^{*}\left(\mathcal{F}_{m}^{k, \varepsilon}\right):=\widetilde{\mathcal{M}}^{*}\left(\mathcal{F}_{m}^{k, \varepsilon}\right) / \mathcal{G}^{2, p}(Y)
$$

is a separable $\mathcal{C}^{k}$ Banach manifold.

We denote $W_{\mathrm{T}_{A} \mathcal{L}}^{1, p}\left(Y, \mathrm{~T}^{*} Y \otimes \mathfrak{g}\right):=\left\{\alpha \in W^{1, p}\left(Y, \mathrm{~T}^{*} Y \otimes \mathfrak{g}\right)|\alpha|_{\partial Y} \in \mathrm{T}_{A} \mathcal{L}\right\}$ and $\mathcal{F}_{m}^{k}:=$ $\mathcal{C}^{k+1}\left(\mathbb{D} \times \mathrm{G}^{m}\right)^{\mathrm{G}}=\mathrm{T}_{f} \mathcal{F}_{m}^{k, \varepsilon}$, and consider the operator

$$
W_{\mathrm{T}_{A} \mathcal{L}}^{1, p}\left(Y, \mathrm{~T}^{*} Y \otimes \mathfrak{g}\right) \times W^{1, p}(Y, \mathfrak{g}) \times \mathcal{F}_{m}^{k} \rightarrow L^{p}\left(Y, \mathrm{~T}^{*} Y \otimes \mathfrak{g}\right) \times L^{p}(Y, \mathfrak{g})
$$

given by

$$
(\alpha, \varphi, \widehat{f}) \mapsto\left(* \mathrm{~d}_{A} \alpha+* \mathrm{~d} X_{f}(A) \alpha-\mathrm{d}_{A} \varphi+* X_{\widehat{f}}(A),-\mathrm{d}_{A}^{*} \alpha\right) .
$$


This operator is $\mathcal{H}_{A} \times(* X .(A), 0)$ and hence it is the linearized operator of $\widetilde{\mathcal{M}}^{*}\left(\mathcal{F}^{k, \varepsilon}\right)$ together with the local slice condition for the $\mathcal{G}^{2, p}(Y)$-action. (The nonlinear operator is a $\mathcal{C}^{k}$ map since the map $* X_{f}: \mathcal{A}^{1, p}(Y) \rightarrow L^{p}\left(Y, \mathrm{~T}^{*} Y \otimes \mathfrak{g}\right)$ is $\mathcal{C}^{k}$ for $f \in \mathcal{C}^{k+1}$.) We must prove that this operator is surjective for every pair $(A, f) \in \mathcal{M}^{*}\left(\mathcal{F}_{m}^{k, \varepsilon}\right)$ when $\varepsilon$ is sufficiently small. We first prove this for $f=f_{0}$. Suppose, by contradiction that there is a nontrivial critical point $A \in \operatorname{Crit}\left(\mathcal{C S}_{\mathcal{L}}+h_{f_{0}}\right)$ such that the operator (91) is not onto. Then with $q^{-1}=1-p^{-1}$ there is a nonzero element

$$
(\eta, \xi) \in L^{q}\left(Y, \mathrm{~T}^{*} Y \otimes \mathfrak{g}\right) \times L^{q}(Y, \mathfrak{g})
$$

orthogonal to the image of (91). Any such element satisfies

$$
\begin{gathered}
\mathrm{d}_{A} \xi=0, \quad * \mathrm{~d}_{A} \eta+* \mathrm{~d} X_{f_{0}}(A) \eta=0, \quad \mathrm{~d}_{A}^{*} \eta=0, \\
\mathrm{~d} h_{\hat{f}}(A) \eta=\int_{Y}\left\langle X_{\widehat{f}}(A) \wedge \eta\right\rangle=0
\end{gathered}
$$

for every $\hat{f} \in \mathcal{F}_{m, \varepsilon}^{k}$. This implies $\xi=0$ because $A$ was assumed to be irreducible. Since $\eta \neq 0$ it follows from Step 1 that $\mathrm{d} \sigma(A) \eta \neq 0$ and hence the map $\mathbb{R} \rightarrow \mathrm{G}^{m} / \mathrm{G}$ : $r \mapsto[\rho(0, A+r \eta)]$ is an embedding into the free part of the quotient near $r=0$. This implies that there exists a map $\hat{f} \in \mathcal{F}_{m}^{k}$ such that

$$
\widehat{f}(z, \rho(z, A+r \eta))=r \beta(r) \beta(|z|),
$$

where $\beta: \mathbb{R} \rightarrow[0,1]$ is a smooth cutoff function that is supported in a sufficiently small neighbourhood of 0 and is equal to 1 near 0 . Hence

$$
\mathrm{d} h_{\widehat{f}}(A) \eta=\left.\frac{\mathrm{d}}{\mathrm{d} r}\right|_{r=0} \int_{\mathbb{D}} \hat{f}(z, \rho(z, A+r \eta)) \mathrm{d}^{2} z=\int_{\mathbb{D}} \beta(|z|) \mathrm{d}^{2} z>0
$$

in contradiction to (92). This proves that the operator (91) is onto whenever $f=f_{0}$ and $[A] \neq[0]$. That this continues to hold for $\left\|f-f_{0}\right\|_{\mathcal{C}^{k+1}}$ sufficiently small follows from compactness and the fact that the trivial connection is nondegenerate.

\section{Step 3 We prove (ii).}

By Step 2, the projection $\mathcal{M}^{*}\left(\mathcal{F}_{m}^{k, \varepsilon}\right) \rightarrow \mathcal{F}_{m}^{k, \varepsilon}$ is a $C^{k}$ Fredholm map of Fredholm index zero. (Its linearization $\operatorname{ker}\left(\mathcal{H}_{A}+(* X .(A), 0) \rightarrow \mathrm{T} \mathcal{F}_{m}^{k, \varepsilon}\right.$ has the same index as the self-adjoint operator $\mathcal{H}_{A}$.) Hence it follows from the Sard-Smale theorem that the set of regular values of this projection is dense in $\mathcal{F}_{m}^{k, \varepsilon}$. For such a regular value $f \in \mathcal{F}_{m}^{k, \varepsilon}$ we have $\operatorname{im}(* X .(A), 0) \subset \operatorname{im} \mathcal{H}_{A}$, so by the surjectivity in Step 2, the operator $\mathcal{H}_{A}$ itself is surjective and hence injective. This shows that $H_{A, f}^{1}=0$ for all critical points $A \in \operatorname{Crit}\left(\mathcal{C S}_{\mathcal{L}}+h_{f}\right)$. For $\left\|f-f_{0}\right\|_{\mathcal{C}^{2}}$ sufficiently small we also have $H_{A}^{0}=0$ by (i), and hence $f$ is "regular" in the sense that Definition 8.2 (i) is satisfied. So we have 
seen that $f_{0} \in \mathcal{F}_{m}$ can be approximated by a sequence of "regular" $\mathcal{C}^{k+1}$ perturbations $f^{v} \in \mathcal{F}_{m}^{k}$ and due to (i) also by a sequence of "regular" smooth perturbations. This proves the theorem.

Proof of Theorem 8.4 To prove (i) we suppose by contradiction that there is a $\gamma \in \Gamma_{m}$ and a sequence $f^{v} \in \mathcal{F}_{m} \backslash \mathcal{F}_{\text {reg }}(\gamma)$ converging to some $f \in \mathcal{F}_{\text {reg }}(\gamma)$ in the $C^{2}$ topology. By Theorem 8.3 we may assume that each $f^{v}$ satisfies condition (i) in Definition 8.2. Thus there is a sequence $\mathbb{A}^{v} \in \widetilde{\mathcal{M}}\left(A_{-}^{v}, A_{+}^{v} ; X_{f^{v}}\right)$ such that $\delta_{f^{v}}\left(\mathbb{A}^{v}\right) \leq 7$, at most one of the limits $A_{ \pm}^{v}$ is gauge equivalent to the trivial connection, and the operator $\mathcal{D}_{\mathbb{A}^{v}, \delta}$ is not surjective. The sequence $\mathbb{A}^{v}$ has bounded energy by Corollary 6.14 and hence a subsequence converges to a broken Floer trajectory $\left(\mathbb{A}_{1}, \ldots, \mathbb{A}_{\ell}\right)$ by Theorem 7.2. Since $f \in \mathcal{F}_{\text {reg }}(\gamma)$, all moduli spaces with negative index and at least one nontrivial limit connection are empty, and the assertion of Corollary 7.4 is wrong. So neither bubbling nor self-connecting trajectories of [0] can occur in the limit. Hence $\mathcal{D}_{\mathbb{A}_{j}, \delta}$ is surjective for every $j$ and, by gluing (see Theorem 7.7 (i)), the operator $\mathcal{D}_{\mathbb{A}^{\nu}, \delta}$ is surjective for $v$ sufficiently large. This contradiction proves (i).

We prove (ii). By assumption $\mathcal{C S}_{\mathcal{L}}+h_{f_{0}}$ has only finitely many critical points in the configuration space $\mathcal{A}(Y, \mathcal{L}) / \mathcal{G}(Y)$. By Corollary 6.14 the energy of a Floer connecting trajectory is $E=\frac{1}{2} \pi^{2}\left(\eta_{f_{0}}\left(A^{+}\right)-\eta_{f_{0}}\left(A^{-}\right)-\operatorname{dim} H_{A^{-}}^{1}+\operatorname{dim} H_{A^{+}}^{1}+j\right)$, where $j$ is the Fredholm index of the linearized operator. There are finitely many such numbers $E \geq 0$ with $j \leq 7$. We order them as

$$
0 \leq E_{0}<E_{1}<\cdots<E_{\ell} .
$$

Claim Let $j \in\{0, \ldots, \ell-1\}$ and $(\gamma, f) \in \Gamma_{m} \times \mathcal{F}_{m}$ such that

$$
\begin{gathered}
\mathbb{A} \in \widetilde{\mathcal{M}}\left(A^{-}, A^{+} ; X_{f}\right),\left(\left[A^{-}\right],\left[A^{+}\right]\right) \neq(0,0), \\
E_{f}(\mathbb{A}) \leq E_{j}, \delta_{f}(\mathbb{A}) \leq 7 \\
\operatorname{Crit}\left(\mathcal{C} \mathcal{S}_{\mathcal{L}}+h_{f}\right)=\operatorname{Crit}\left(\mathcal{C S}_{\mathcal{L}}+h_{f_{0}}\right) \\
A \in \operatorname{Crit}\left(\mathcal{C S}_{\mathcal{L}}+h_{f_{0}}\right) \Longrightarrow \mathcal{D}_{\mathbb{A}, \delta} \text { is onto } \\
\Longrightarrow \quad h_{f}(A)=h_{f_{0}}(A),
\end{gathered}
$$

Fix an integer $k \in \mathbb{N}$ and a constant $\varepsilon>0$. Then there is a perturbation $\left(\gamma^{\prime}, f^{\prime}\right) \in$ $\Gamma_{m^{\prime}} \times \mathcal{F}_{m^{\prime}}$ satisfying (93) to (95) with $j$ replaced by $j+1$ and

$$
\left\|\left|X_{f^{\prime}}-X_{f}\right|\right\|_{k}<\varepsilon
$$

A connection $\mathbb{A} \in \mathcal{M}\left(A^{-}, A^{+} ; X_{f}\right)$ with energy $E_{f}(\mathbb{A}) \leq 0$ must be gauge equivalent to the constant path $A^{-}=A^{+} \notin[0]$. By assumption these critical points of $\mathcal{C} \mathcal{S}_{\mathcal{L}}+h_{f_{0}}$ are nondegenerate. So by Theorem 3.13 the hypotheses of the claim are satisfied for $j=0$ and $(\gamma, f)=\left(\gamma_{0}, f_{0}\right)$. Therefore assertion (ii) of the theorem follows from the claim by induction on $j$. We prove the claim in four steps. 
Step 1 The quotient of the set

$$
\mathcal{K}:=\bigcup_{\left(\left[A^{-}\right],\left[A^{+}\right] \neq(0,0)\right.}\left\{\begin{array}{l|l}
A: \mathbb{R} \rightarrow \mathcal{A}(Y, \mathcal{L}) & \begin{array}{l}
\partial_{s} A+*\left(F_{A}+X_{f}(A)\right)=0, \\
\lim _{s \rightarrow \pm \infty} A(s) \in\left[A^{ \pm}\right], \\
E_{f}(\mathbb{A}) \leq E_{j+1}, \delta_{f}(\mathbb{A}) \leq 7, \\
\mathcal{D}_{\mathbb{A}, \delta} \text { not onto }
\end{array}
\end{array}\right\}
$$

by the gauge group $\mathcal{G}(Y)$ is compact.

This is proven by the same discussion as in (i). The argument uses in addition the fact that the energy of each limit trajectory $\mathbb{A}_{j}$ is strictly less than the energy of the $\mathbb{A}^{v}$ if bubbling or breaking of trajectories occurs. (So the relevant moduli spaces will be transverse or empty by assumption.)

Step 2 There is a $\gamma^{\prime} \in \Gamma_{m^{\prime}}$ with $\gamma_{i}^{\prime}=\gamma_{i}$ for $i=1, \ldots, m$ satisfying the following conditions. For $z \in \mathbb{D}$ and $A \in \mathcal{A}(Y)$ let $\rho_{i}^{\prime}(z, A)$ be the holonomy of $A$ around the loop $\theta \mapsto \gamma_{i}^{\prime}(\theta, z)$ and define $\sigma: \mathcal{A}(Y) \rightarrow \mathrm{G}^{m^{\prime}}$ by

$$
\sigma(A):=\left(\rho_{1}^{\prime}(0, A), \ldots, \rho_{m^{\prime}}^{\prime}(0, A)\right) .
$$

Then, for every $\mathbb{A} \in \mathcal{K}$, there is an $s_{0} \in \mathbb{R}$ such that the following holds.

(a) The tuple $\sigma\left(A\left(s_{0}\right)\right)$ is not contained in $\sigma\left(\operatorname{Crit}\left(\mathcal{C S}_{\mathcal{L}}+h_{f}\right)\right)$ and belongs to the free part of $\mathrm{G}^{m^{\prime}}$ for the action of $\mathrm{G}$ by simultaneous conjugation. Moreover, $\sigma(A(s)) \not \sigma\left(A\left(s_{0}\right)\right)$ for every $s \in \mathbb{R} \backslash\left\{s_{0}\right\}$.

(b) For every nonzero section $(\eta, 0) \in \operatorname{ker} \mathcal{D}_{\mathbb{A}, \delta}^{*}$ the vectors $\mathrm{d} \sigma\left(A\left(s_{0}\right)\right) \partial_{s} A\left(s_{0}\right)$ and $\mathrm{d} \sigma\left(A\left(s_{0}\right)\right) \eta\left(s_{0}\right)$ are linearly independent in $\mathrm{T}\left(\mathrm{G}^{m^{\prime}} / \mathrm{G}\right)$.

For every $s_{0} \in \mathbb{R}$ and every $\gamma^{\prime}$ the set of all $\mathbb{A} \in \mathcal{K}$ that satisfy conditions (a) and (b) is open. Moreover, (a) and (b) are preserved under gauge transformations and under adding further loops to $\gamma^{\prime}$. So it suffices to establish (a) and (b) for a single element of $\mathcal{K}$. (Then $\mathcal{K}$ is covered by finitely many gauge orbits of small open sets around such elements, and the final $\gamma^{\prime}$ results from taking the union over all loops that are required by these different elements.) Hence from now on we fix an element $\mathbb{A} \in \mathcal{K}$. Since either $A^{+}$or $A^{-}$is irreducible, there is an $s_{0} \in \mathbb{R}$ such that $A\left(s_{0}\right)$ is irreducible. Since the path $s \mapsto\left(\mathrm{d}_{A(s)} \xi, 0\right)$ is a solution of (105) for every $\xi \in \Omega^{1}(Y, \mathfrak{g})$, it follows from Proposition 8.6 (ii) below that

$$
\partial_{s} A\left(s_{0}\right) \notin \operatorname{imd}_{A\left(s_{0}\right)}
$$


otherwise we would have $\partial_{s} A(s)=\mathrm{d}_{A(s)} \xi$ for all $s \in \mathbb{R}$ and, by partial integration, $\left\|\mathrm{d}_{A} \xi\right\|_{L^{2}(Y)}=-\int_{Y}\left\langle\mathrm{~d}_{A} \xi \wedge\left(F_{A}+X_{f}(A)\right)\right\rangle=0$ which would imply $\partial_{s} A \equiv 0$ and hence $E_{f}(\mathbb{A})=0$. By Proposition 8.6 (i) below, we have that

$$
A\left(s_{0}\right) \notin \bigcup_{s \neq s_{0}}[A(s)] \cup \operatorname{Crit}\left(\mathcal{C S}_{\mathcal{L}}+h_{f}\right) ;
$$

otherwise $A: \mathbb{R} \rightarrow \mathcal{A}(Y, \mathcal{L})$ would be constant or periodic modulo gauge, in contradiction to $0<E_{f}(\mathbb{A})<\infty$. Moreover, for $(\eta, 0) \in \operatorname{ker} \mathcal{D}_{\mathbb{A}, \delta}^{*}$, we have

$$
\eta\left(s_{0}\right) \perp \mathbb{R} \partial_{S} A\left(s_{0}\right)+\operatorname{imd}_{A\left(s_{0}\right)} .
$$

To see this, fix an element $\xi \in \Omega^{0}(Y, \mathfrak{g})$. Then $\alpha(s):=\partial_{s} A(s)+\mathrm{d}_{A(s)} \xi$ and $\eta(s)$ satisfy the differential equations

$$
\partial_{s} \alpha+*\left(\mathrm{~d}_{A} \alpha+\mathrm{d} X_{f}(A) \alpha\right)=0, \quad \partial_{s} \eta+2 \partial_{s} V \eta-*\left(\mathrm{~d}_{A} \eta+\mathrm{d} X_{f}(A) \eta\right)=0,
$$

and the Lagrangian boundary condition $\left.\eta(s)\right|_{\partial Y},\left.\alpha(s)\right|_{\partial Y} \in T_{A(s)} \mathcal{L}$. Hence

$$
\frac{\mathrm{d}}{\mathrm{d} s} \exp (2 V) \int_{Y}\langle\eta, \alpha\rangle=\exp (2 V)\left(\int_{Y}\left\langle\partial_{s} \eta+2 \partial_{s} V \eta, * \alpha\right\rangle+\int_{Y}\left\langle\eta, * \partial_{s} \alpha\right\rangle\right)=0 .
$$

The last identity uses the fact that the operator $\alpha \mapsto *\left(\mathrm{~d}_{A} \alpha+\mathrm{d} X_{f}(A) \alpha\right)$ with the Lagrangian boundary condition is self-adjoint for every $s$. Since the inner product $e^{2 V} \int_{Y}\langle\eta, \alpha\rangle$ converges to zero for $s \rightarrow \pm \infty$, this proves (99).

As in the proof of Theorem 8.3 we shall use Remark 8.5 to construct $\gamma^{\prime}$ and it suffices in each step to find the loop $\theta \mapsto \gamma_{i}^{\prime}(\theta, 0)$. Since $A\left(s_{0}\right)$ is irreducible and using (97) we can argue exactly as in the proof of Step 1 in Theorem 8.3, with $(A, \eta)$ replaced by $\left(A\left(s_{0}\right), \partial_{s} A\left(s_{0}\right)\right)$, to prove that $\gamma^{\prime}$ can be chosen such that $\sigma\left(A\left(s_{0}\right)\right)$ belongs to the free part of $\mathrm{G}^{m^{\prime}}$ and

$$
\mathrm{d} \sigma\left(A\left(s_{0}\right)\right) \partial_{s} A\left(s_{0}\right) \notin V_{0},
$$

where $V_{0} \subset \mathrm{T}_{\sigma\left(A\left(s_{0}\right)\right)} \mathrm{G}^{m^{\prime}}$ is the tangent space of the $\mathrm{G}$-orbit through $\sigma\left(A\left(s_{0}\right)\right)$, namely

$$
V_{0}:=\left\{v=\left(\sigma_{i}\left(A\left(s_{0}\right)\right) \xi_{0}-\xi_{0} \sigma_{i}\left(A\left(s_{0}\right)\right)\right)_{i=1, \ldots, m^{\prime}} \mid \xi_{0} \in \mathfrak{g}\right\} .
$$

This implies that $\left[\mathrm{d} \sigma\left(A\left(s_{0}\right)\right) \partial_{s} A\left(s_{0}\right)\right] \neq 0$ in the tangent space of the quotient $\mathrm{G}^{m^{\prime}} / \mathrm{G}$. It follows that the curve $\left[s_{0}-\delta, s_{0}+\delta\right] \rightarrow \mathrm{G}^{m^{\prime}} / \mathrm{G}: s \mapsto[\sigma(A(s))]$ is injective for $\delta>0$ sufficiently small. The set

$$
\mathcal{C}:=\left\{[A(s)]|| s-s_{0} \mid \geq \delta\right\} \cup \operatorname{Crit}\left(\mathcal{C S}_{\mathcal{L}}+h_{f}\right) / \mathcal{G}(Y) \subset \mathcal{A}(Y) / \mathcal{G}(Y)
$$

is compact and, by (98), does not contain $\left[A\left(s_{0}\right)\right]$. Now (i) holds if and only if $\sigma(B) \not \sigma\left(A\left(s_{0}\right)\right)$ for every $[B] \in \mathcal{C}$. Since this condition is open in $B$, and $\mathcal{C}$ is 
compact, it suffices to prove this for a fixed element $[B] \in \mathcal{C}$. Given $[B] \in \mathcal{C}$ it follows from Remark 8.5 (i) that for every $g \in \mathrm{G}$ there is a based loop $\gamma$ such that

$$
\rho_{\gamma}(B) \neq g^{-1} \rho_{\gamma}\left(A\left(s_{0}\right)\right) g .
$$

For every fixed loop $\gamma$ this condition is open in $g$. Since $\mathrm{G}$ is compact there exist finitely many loops $\gamma_{i}^{\prime}$ such that the tuple $\left(\rho_{\gamma_{i}^{\prime}}(B)\right)_{i}$ is not simultaneously conjugate to $\left(\rho_{\gamma_{i}^{\prime}}\left(A\left(s_{0}\right)\right)\right)_{i}$. For this choice of the loops $\gamma_{i}^{\prime}$ we have that $\sigma(B) \nsim \sigma\left(A\left(s_{0}\right)\right)$ as claimed.

To prove (b) it suffices to consider a fixed nonzero element $(\eta, 0) \in \operatorname{ker} \mathcal{D}_{\mathbb{A}, \delta}^{*}$ because this kernel is finite dimensional. Since $\eta\left(s_{0}\right) \neq 0$ (by unique continuation as in Proposition 8.6 (ii)) it follows from (99) that

$$
\eta\left(s_{0}\right)-\lambda \partial_{s} A\left(s_{0}\right) \notin \operatorname{imd}_{A\left(s_{0}\right)} \quad \forall \lambda \in \mathbb{R} .
$$

By (100) we have $\delta:=\inf _{v \in V_{0}} \mid \mathrm{d} \sigma\left(A\left(s_{0}\right) \partial_{s} A\left(s_{0}\right)-v \mid>0\right.$ and

$$
\mathrm{d} \sigma\left(A\left(s_{0}\right)\right)\left(\eta\left(s_{0}\right)-\lambda \partial_{s} A\left(s_{0}\right)\right) \notin V_{0}
$$

for $|\lambda|>\delta^{-1} \| \mathrm{d} \sigma\left(A\left(s_{0}\right) \eta\left(s_{0}\right) \|=: c\right.$. We wish prove that (101) continues to hold for all $\lambda \in[-c, c]$ with a suitable choice of $\gamma^{\prime}$. For each fixed $\lambda$ the proof is the same as that of Step 1 in the proof of Theorem 8.3. Since condition (101) is open in $\lambda$ this proves Step 2.

Step 3 Let $C:=\left\{\left(z, \rho^{\prime}(z, A)\right) \in \mathbb{D} \times \mathrm{G}^{m^{\prime}} \mid A \in \operatorname{Crit}\left(\mathcal{C} \mathcal{S}_{\mathcal{L}}+h_{f}\right)\right\}$. For $\varepsilon^{\prime}>0$ and $k \in \mathbb{N}$ (possibly larger than the constant in the claim) denote

$$
\mathcal{F}_{m^{\prime}}^{k, \varepsilon^{\prime}}:=\left\{f^{\prime} \in \mathcal{C}^{k+1}\left(\mathbb{D} \times \mathrm{G}^{m^{\prime}}\right)^{\mathrm{G}}\left|\left(f^{\prime}-f\right)\right|_{B_{\varepsilon^{\prime}}(C)} \equiv 0,\left\|f^{\prime}-f\right\|_{\mathcal{C}^{k+1}}<\varepsilon^{\prime}\right\}
$$

and for a fixed $p>4$ let

$$
\widetilde{\mathcal{M}}\left(A^{-}, A^{+}, \mathcal{F}_{m^{\prime}}^{k, \varepsilon^{\prime}}\right):=\left\{\begin{array}{l|l}
\left(\mathbb{A}, f^{\prime}\right) \in \mathcal{A}_{\delta}^{1, p} \times \mathcal{F}_{m^{\prime}}^{k, \varepsilon^{\prime}} & \begin{array}{l}
\mathbb{A} \in \widetilde{\mathcal{M}}\left(A^{-}, A^{+} ; X_{f^{\prime}}\right) \\
E_{f^{\prime}}(\mathbb{A}) \leq E_{j+1} \\
\delta_{f^{\prime}}(\mathbb{A}) \leq 7
\end{array}
\end{array}\right\} .
$$

Here we abbreviate $\mathcal{A}_{\delta}^{1, p}:=\mathcal{A}_{\delta}^{1, p}\left(\mathbb{R} \times Y, \mathcal{L} ; A^{-}, A^{+}\right.$) (see Equation (74)). Let $\mathcal{G}_{0}^{2, p}(\mathbb{R} \times Y)$ be the $W^{2, p}$-closure of $\{u: \mathbb{R} \rightarrow \mathcal{G}(Y)|u(s)=1 \quad \forall| s \mid \geq 1\}$. Then for every $k \in \mathbb{N}$ there is an $\varepsilon^{\prime}>0$ such that the following holds.

Every perturbation $f^{\prime} \in \mathcal{F}_{m^{\prime}}^{k, \varepsilon^{\prime}}$ satisfies conditions (94), (95), (96), and for every pair of critical points $\left(\left[A^{-}\right],\left[A^{+}\right]\right) \neq(0,0)$ the universal moduli space

$$
\mathcal{M}\left(A^{-}, A^{+}, \mathcal{F}_{m^{\prime}}^{k, \varepsilon^{\prime}}\right):=\widetilde{\mathcal{M}}\left(A^{-}, A^{+}, \mathcal{F}_{m^{\prime}}^{k, \varepsilon^{\prime}}\right) / \mathcal{G}_{0}^{2, p}(\mathbb{R} \times Y)
$$

is a separable $\mathcal{C}^{k}$-Banach manifold. 
Conditions (94), (95), and (96) are satisfied for every $f^{\prime} \in \mathcal{F}_{m^{\prime}}^{k, \varepsilon^{\prime}}$ for $\varepsilon^{\prime}>0$ sufficiently small. The assertion about the universal moduli space holds whenever the linearized operator

$$
(\alpha, \varphi, \widehat{f}) \mapsto \mathcal{D}_{\mathbb{A}, \delta}(\alpha, \varphi)+\left(X_{\widehat{f}}(\mathbb{A}), 0\right)
$$

is surjective for every pair $\left(\mathbb{A}, f^{\prime}\right) \in \widetilde{\mathcal{M}}\left(A^{-}, A^{+}, \mathcal{F}_{m^{\prime}}^{k, \varepsilon^{\prime}}\right)$. Here $\mathcal{D}_{\mathbb{A}, \delta}$ is the operator (71) with $k=1$. We first prove that this holds for $f^{\prime}=f$. If $\mathbb{A}$ is not gauge equivalent (by $\mathcal{G}\left(A^{-}, A^{+}\right)$) to a connection in $\mathcal{K}$, then the operator $\mathcal{D}_{\mathbb{A}, \delta}$ is surjective by Remark 6.10 (i), and hence so is (102). Let $\mathbb{A} \in \mathcal{K}$ (after a gauge transformation in $\mathcal{G}\left(A^{-}, A^{+}\right)$) and $q^{-1}:=1-p^{-1}$, and suppose, by contradiction, that there is a nonzero pair

$$
(\eta, \varphi) \in L_{\delta}^{q}\left(\mathbb{R} \times Y, \mathrm{~T}^{*} Y \otimes \mathfrak{g}\right) \times L_{\delta}^{q}(\mathbb{R} \times Y, \mathfrak{g})
$$

orthogonal to the image of (102). Then we have $\varphi=0$ (by the proof of Theorem 6.9), $\eta \in W_{\mathbb{A}}^{1, p}\left(\mathbb{R} \times Y, \mathrm{~T}^{*} Y \otimes \mathfrak{g}\right)$ (by Theorem 3.11), $\mathcal{D}_{\mathbb{A}, \delta}^{*}(\eta, 0)=0$, and

$$
\int_{-\infty}^{\infty} \exp (2 V(s)) \mathrm{d} h_{\widehat{f}}(A(s)) \eta(s) \mathrm{d} s=0
$$

for every $\hat{f} \in \mathrm{T}_{f} \mathcal{F}_{m^{\prime}}^{k, \varepsilon^{\prime}}$. By Step 2 there is $s_{0} \in \mathbb{R}$ such that $\sigma(A(s)) \neq \sigma\left(A\left(s_{0}\right)\right)$ for $s \neq s_{0}$ and the tangent vectors $\mathrm{d} \sigma\left(A\left(s_{0}\right)\right) \partial_{s} A\left(s_{0}\right), \mathrm{d} \sigma\left(A\left(s_{0}\right)\right) \eta\left(s_{0}\right)$ are linearly independent. Hence the map

$$
(r, s) \mapsto \rho(z, A(s)+r \eta(s))
$$

is an embedding in a neighbourhood of $\left(0, s_{0}\right) \in \mathbb{R}^{2}$ for every sufficiently small $z \in \mathbb{D}$. It follows that there exists a smooth G-invariant map $\hat{f}: \mathbb{D} \times \mathrm{G}^{m^{\prime}} \rightarrow \mathbb{R}$ vanishing in a neighbourhood of $C$ and satisfying

$$
\widehat{f}(z, \rho(z, A(s)+r \eta(s)))=r \beta(r) \beta\left(s-s_{0}\right) \beta(|z|)
$$

for a suitable cutoff function $\beta: \mathbb{R} \rightarrow[0,1]$ that is supported in a neighbourhood of 0 and is equal to 1 near 0 . This implies

$$
\begin{aligned}
d h_{\hat{f}}(A(s)) \eta(s) & =\left.\int_{\mathbb{D}} \frac{\partial}{\partial r}\right|_{r=0} \hat{f}(z, \rho(z, A(s)+r \eta(s))) \mathrm{d}^{2} z \\
& =\beta\left(s-s_{0}\right) \int_{\mathbb{D}} \beta(|z|) \mathrm{d}^{2} z \geq 0
\end{aligned}
$$

for every $s \in \mathbb{R}$. Hence the integral on the right hand side of (103) does not vanish, contradiction. Thus we have proved that the operator (102) is onto whenever $f^{\prime}=f$. 
We must prove that (102) is onto when $\left\|f^{\prime}-f\right\|_{\mathcal{C}^{k+1}}$ is sufficiently small. Otherwise there are sequences $\mathcal{F}_{m^{\prime}}^{k, \varepsilon^{\prime}} \ni f^{v} \rightarrow f$ and $\mathbb{A}^{v} \in \widetilde{\mathcal{M}}\left(A^{-}, A^{+} ; X_{f^{v}}\right)$ such that the operator $(102)$, with $\left(\mathbb{A}, f^{\prime}\right)$ replaced by $\left(\mathbb{A}^{v}, f^{v}\right)$, is not onto. If $\mathbb{A}^{v}$ converges (modulo gauge) to $\mathbb{A} \in \mathcal{K}$ then (102) is surjective for the pair $(\mathbb{A}, f)$ and hence for $\left(A^{v}, f^{v}\right)$ when $v$ is sufficiently large. Otherwise it follows from the compactness and gluing theorems as in the proof of (i) that $\mathcal{D}_{\mathbb{A}^{v}, \delta}$ is surjective for $v$ sufficiently large. This contradiction finishes the proof of Step 3.

Step 4 We prove the claim.

By Step 3 the projection $\mathcal{M}\left(A^{-}, A^{+}, \mathcal{F}_{m^{\prime}}^{k, \varepsilon^{\prime}}\right) \rightarrow \mathcal{F}_{m^{\prime}}^{k, \varepsilon^{\prime}}$ is a Fredholm map of index at most 7 for every pair $A^{ \pm} \in \operatorname{Crit}\left(\mathcal{C S}_{\mathcal{L}}+h_{f}\right)$ with $\left(\left[A^{-}\right],\left[A^{+}\right]\right) \neq(0,0)$. (The index at $(\mathbb{A}, f)$ is the same as that of the linearized operator $\mathcal{D}_{\mathbb{A}, \delta}$.) Hence it follows from the Sard-Smale theorem that, for $k \geq 8$, the set of regular values is of the second category in the sense of Baire. Any such regular value $f \in \mathcal{F}_{m^{\prime}}^{k, \varepsilon^{\prime}}$ satisfies (93). To prove the claim, pick a regular value of the projection and approximate it by a smooth perturbation $f^{\prime}$. In the last step we use the fact that the set of all perturbations that satisfy the requirements of the claim is open in the $\mathcal{C}^{k+1}$-topology. (The proof is analogous to the proof of (i).) This proves the theorem.

The main difference between our proof of Theorem 8.4 and the argument in Donaldson's book [10, p 144] for the closed case is that we do not have a gluing theorem converse to bubbling on the boundary and hence cannot work on a compact part of the moduli space in the presence of bubbling on the boundary. To circumvent this difficulty we have restricted the discussion to the monotone case and to Floer connecting trajectories of index less than or equal to seven. We also made use of a unique continuation result for perturbed anti-self-dual connections with Lagrangian boundary conditions, which is established next.

\section{Unique continuation}

Proposition 8.6 Let $(\gamma, f) \in \Gamma_{m} \times \mathcal{F}_{m}$ and fix an open interval $I \subset \mathbb{R}$.

(i) Let $A, B: I \rightarrow \mathcal{A}(Y)$ be two solutions of the Floer equation

$$
\partial_{s} A+* F_{A}+* X_{f}(A)=0,\left.\quad A(s)\right|_{\Sigma} \in \mathcal{L} .
$$

If $A\left(s_{0}\right)=B\left(s_{0}\right)$ for some $s_{0} \in I$ then $A(s)=B(s)$ for all $s \in I$.

(ii) Let $A: I \rightarrow \mathcal{A}(Y, \mathcal{L})$ and $\xi=(\alpha, \varphi): I \rightarrow \Omega^{1}(Y, \mathfrak{g}) \times \Omega^{0}(Y, \mathfrak{g})$ be smooth maps satisfying the (augmented) linearized Floer equation

$$
\partial_{s} \xi+\mathcal{H}_{A} \xi=0,\left.\quad \alpha(s)\right|_{\Sigma} \in \mathrm{T}_{A(s)} \mathcal{L},\left.\quad * \alpha(s)\right|_{\Sigma}=0 .
$$


If $\xi\left(s_{0}\right)=0$ for some $s_{0} \in I$ then $\xi(s)=0$ for all $s \in I$.

The proof will use the following local continuation result in the interior. This was proven by Taubes [31] in a slightly different formulation; we include the proof for the sake of completeness.

Lemma 8.7 Let $U$ be a (not necessarily compact) 3-manifold without boundary and $I \subset \mathbb{R}$ be an open interval.

(i) Let $A, B: I \rightarrow \mathcal{A}(U)$ be two solutions of the unperturbed Floer equation (104) with $f=0$. If $A\left(s_{0}\right)=B\left(s_{0}\right)$ for some $s_{0} \in I$ then $A(s)=B(s)$ for all $s \in I$.

(ii) Let $A: I \rightarrow \mathcal{A}(U)$ and $\xi=(\alpha, \varphi): I \rightarrow \Omega^{1}(U, \mathfrak{g}) \times \Omega^{0}(U, \mathfrak{g})$ be smooth maps satisfying the unperturbed linearized Floer equation (105) with $f=0$. If $\xi\left(s_{0}\right)=0$ for some $s_{0} \in I$ then $\xi(s)=0$ for all $s \in I$.

Proof To prove (i) assume by contradiction that $A\left(s^{\prime}, y_{1}\right) \neq B\left(s^{\prime}, y_{1}\right)$ for some $\left(s^{\prime}, y_{1}\right) \in I \times U$. Let $D_{r}\left(y_{1}\right) \subset U$ be a geodesic ball of radius $r>0$ around $y_{1}$ and denote

$$
J:=\left\{s \in I|A(s)|_{D_{r / 2}\left(y_{1}\right)}=\left.B(s)\right|_{D_{r / 2}\left(y_{1}\right)}\right\} \subset I .
$$

This set contains $s_{0}$ by assumption and it is a closed subset of $I$ because $A-B$ is continuous. We claim that $J \subset I$ is open and hence $J=I$ in contradiction to the assumption.

To prove that $J$ is open we fix an element $s_{1} \in J$. Then $A-B$ vanishes to infinite order (ie with all derivatives) at $x_{1}:=\left(s_{1}, y_{1}\right)$. For the derivatives in the direction of $I$ this follows from the Floer equation. Let $D_{r}\left(x_{1}\right) \subset I \times U$ denote the geodesic ball centred at $x_{1}$. We fix gauge transformations $u_{A}, u_{B} \in \mathcal{G}\left(D_{r}\left(x_{1}\right)\right)$ with $u_{A}\left(x_{1}\right)=u_{B}\left(x_{1}\right)=1$ such that $u_{A}^{*} A$ and $u_{B}^{*} B$ are in radial gauge on $D_{r}\left(x_{1}\right)$. Then these can be pulled back to connections in temporal gauge $A^{\prime}, B^{\prime}:(-\infty, \log r) \rightarrow \mathcal{A}\left(S^{3}\right)$ by geodesic polar coordinates $(-\infty, \log r) \times S^{3} \stackrel{\sim}{\rightarrow} D_{r}\left(x_{1}\right) \backslash\left\{x_{1}\right\}$. The fact that $u_{A}^{*} A-u_{B}^{*} B$ vanishes to infinite order at $x_{1}$ translates into superexponential convergence $A^{\prime}(s)-B^{\prime}(s) \rightarrow 0$ as $s \rightarrow-\infty$. In particular, for every $K>0$, we have

$$
\lim _{s \rightarrow-\infty} e^{-K s}\left\|A^{\prime}(s)-B^{\prime}(s)\right\|_{L^{2}\left(S^{3}\right)}=0 .
$$

The pullback metric on $(-\infty, \log r) \times S^{3}$ has the form $e^{2 s}\left(\mathrm{~d} s^{2}+g_{s}\right)$, where $g$ is a smooth family of metrics on $S^{3}$ that converges exponentially to the standard metric on $S^{3}$ as $s \rightarrow-\infty$. Since the anti-self-duality equation is conformally invariant, the connections $A^{\prime}$ and $B^{\prime}$ also satisfy (104) with respect to the metric $\mathrm{d} s^{2}+g_{s}$ on $(-\infty, \log r) \times S^{3}$. We now denote $\alpha:=B^{\prime}-A^{\prime}:(-\infty, \log r) \rightarrow \Omega^{1}\left(S^{3}, \mathfrak{g}\right)$ and use 
the technique of Agmon-Nirenberg in Appendix $\mathrm{C}$ to prove that $\alpha \equiv 0$. The Floer equations (ie the anti-self-duality of $A^{\prime}$ and $B^{\prime}$ w.r.t. the conformally rescaled metric) imply that $\alpha$ satisfies

$$
\partial_{S} \alpha+* \mathrm{~d}_{A^{\prime}+\frac{1}{2} \alpha} \alpha=0 .
$$

We shall use the operator $\mathbb{F}:=-* \mathrm{~d}_{A^{\prime}+\frac{1}{2} \alpha}$ (corresponding to $A(s)$, appropriately shifted, in the notation of Appendix $C$ ) which is self-adjoint with respect to the time dependent inner product

$$
\langle\alpha, \beta\rangle_{s}:=\int_{S^{3}}\left\langle\alpha \wedge *_{s} \beta\right\rangle=\langle Q(s) \alpha, Q(s) \beta\rangle_{E} .
$$

Here $*_{s}$ is the Hodge operator for the metric $g_{s}$ on $S^{3}$, and the subscript $E$ indicates the use of the standard metric on $S^{3}$. The operator $Q(s): \Omega^{1}\left(S^{3}, \mathfrak{g}\right) \rightarrow \Omega^{1}\left(S^{3}, \mathfrak{g}\right)$ is defined as in [11, p 151], as a self-adjoint operator such that $Q(s)^{2}=*_{E} *_{s}$. This square root exists since $*_{E} *_{s}$ is positive definite. These operators satisfy (Q1) in Appendix $\mathrm{C}$ by the exponential convergence of $g_{s}$ as $s \rightarrow-\infty$. Moreover,

$$
\begin{aligned}
-\frac{\mathrm{d}}{\mathrm{d} s}\left\langle\alpha, * \mathrm{~d}_{A^{\prime}+\frac{1}{2} \alpha} \alpha\right\rangle_{S}+2\left\langle\partial_{S} \alpha, * \mathrm{~d}_{A^{\prime}+\frac{1}{2} \alpha} \alpha\right\rangle_{S} & =-\int_{S^{3}}\left\langle\alpha \wedge\left[\partial_{S}\left(A^{\prime}+\frac{1}{2} \alpha\right) \wedge \alpha\right]\right\rangle \\
& \leq\left\|\partial_{S} A^{\prime}+\frac{1}{2} \partial_{S} \alpha\right\|_{L^{\infty}\left(S^{3}\right)}\|\alpha\|_{S}^{2} .
\end{aligned}
$$

Hence the function $x(s):=\alpha\left(s_{2}-s\right)$, with $s_{2} \in(-\infty, \log r)$, satisfies the assumptions of Theorem C.2 with $c_{1}=c_{2}=0$ and $c_{3}(s)=\left\|\partial_{s} A^{\prime}+\frac{1}{2} \partial_{s} \alpha\right\|_{L^{\infty}\left(S^{3}\right)}$. The constant $c$ in Theorem C.2 is finite because

$$
\int_{-\infty}^{s_{2}}\left\|\partial_{S} A^{\prime}+\frac{1}{2} \partial_{s} \alpha\right\|_{L^{\infty}\left(S^{3}\right)}<\infty
$$

by the exponential decay of $A^{\prime}$ and $B^{\prime}$ (see Theorem 5.1). We thus obtain

$$
\|\alpha(s)\|_{s} \geq e^{-c\left(s_{2}-s\right)}\left\|\alpha\left(s_{2}\right)\right\|_{s_{2}}
$$

for all $s \in\left(-\infty, s_{2}\right]$. This estimate contradicts the superexponential convergence in (106) unless $\alpha\left(s_{2}\right)=0$. Since $s_{2}$ is any element of the interval $(-\infty, \log r)$ we have shown that $\alpha \equiv 0$ and hence $u_{A}^{*} A=u_{B}^{*} B$ on the geodesic ball $D_{r}\left(x_{1}\right)$ around $x_{1}=\left(s_{1}, y_{1}\right)$. This ball contains the set $\left[s_{1}-\frac{r}{2}, s_{1}+\frac{r}{2}\right] \times D_{r / 2}\left(y_{1}\right)$. From the construction of the gauge transformations with $A=B$ on $\left\{s_{1}\right\} \times D_{r / 2}\left(y_{1}\right)$ we know that $\left.u_{A}\right|_{s=s_{1}}=\left.u_{B}\right|_{s=s_{1}}$. Now there is a unique gauge transformation $v$ on $\left[s_{1}-\frac{r}{2}, s_{1}+\frac{r}{2}\right] \times D_{r / 2}\left(y_{1}\right)$ with $\left.v\right|_{s=s_{1}}=\left.u_{A}^{-1}\right|_{s=s_{1}}=\left.u_{B}^{-1}\right|_{s=s_{1}}$ that puts $u_{A}^{*} A=u_{B}^{*} B$ back into temporal gauge. By the uniqueness of the temporal gauge with $\left.u_{A} v\right|_{s=s_{1}}=$ $\left.u_{B} v\right|_{s=s_{1}}=11$ this implies

$$
A=\left(u_{A} v\right)^{*} A=\left(u_{B} v\right)^{*} B=B \quad \text { on } \quad\left[s_{1}-\frac{r}{2}, s_{1}+\frac{r}{2}\right] \times D_{r / 2}\left(y_{1}\right)
$$


and hence $\left[s_{1}-\frac{r}{2}, s_{1}+\frac{r}{2}\right] \subset J$. This proves that $J$ is open as claimed.

The proof of (ii) is analogous to (i). In conformal polar coordinates near $x_{1}$ we choose the radial gauge $u_{A}^{*} A$ as before. The pullback $\xi^{\prime}:(-\infty, \log r) \rightarrow \Omega^{1}\left(S^{3}, \mathfrak{g}\right) \times$ $\Omega^{0}\left(S^{3}, \mathfrak{g}\right)$ then satisfies the linearized Floer equation with respect to $A^{\prime}$. Now the Agmon-Nirenberg technique for $x=\xi^{\prime}$ (with the Hessian $\mathcal{H}_{A^{\prime}(s)}$ as self-adjoint operator) shows that $\xi^{\prime} \equiv 0$ and hence $\xi=0$ on $D_{r}\left(x_{1}\right)$. The relevant estimate is

$$
\begin{aligned}
-\frac{\mathrm{d}}{\mathrm{d} s} & \left\langle\xi^{\prime}, \mathcal{H}_{A^{\prime}} \xi^{\prime}\right\rangle_{S}+2\left\langle\partial_{s} \xi^{\prime}, \mathcal{H}_{A^{\prime}} \xi^{\prime}\right\rangle_{S} \\
& =-\frac{\mathrm{d}}{\mathrm{d} s}\left(\int_{S^{3}}\left\langle\alpha^{\prime} \wedge \mathrm{d}_{A^{\prime}} \alpha^{\prime}\right\rangle-2 \int_{S^{3}}\left\langle\alpha^{\prime} \wedge * \mathrm{~d}_{A^{\prime}} \varphi^{\prime}\right\rangle\right)+2\left\langle\partial_{S}\left(\alpha^{\prime}, \varphi^{\prime}\right), \mathcal{H}_{A^{\prime}}\left(\alpha^{\prime}, \varphi^{\prime}\right)\right\rangle_{S} \\
& =-\int_{S^{3}}\left\langle\alpha^{\prime} \wedge\left[\partial_{S} A^{\prime}, \alpha^{\prime}\right]\right\rangle+2 \int_{S^{3}}\left\langle\alpha^{\prime} \wedge *\left[\partial_{S} A^{\prime}, \varphi^{\prime}\right]\right\rangle+2 \int_{S^{3}}\left\langle\alpha^{\prime} \wedge\left(\partial_{S} *\right) \mathrm{d}_{A^{\prime}} \varphi^{\prime}\right\rangle \\
& \leq 2 c_{2}(s)\left\|\mathcal{H}_{A^{\prime}} \xi^{\prime}\right\|_{S}\left\|\xi^{\prime}\right\|_{S}+c_{3}(s)\left\|\xi^{\prime}\right\|_{S}^{2},
\end{aligned}
$$

where $c_{2}(s)=2 \delta^{-1} c_{Q}(s)$ and

$$
c_{3}(s)=2\left\|\partial_{S} A^{\prime}\right\|_{L^{\infty}\left(S^{3}\right)}+8 \delta^{-1} c_{Q}(s)\left\|F_{A^{\prime}}\right\|_{L^{\infty}\left(S^{3}\right)}^{1 / 2}
$$

with $\delta$ and $c_{Q}$ as in (Q1) in Appendix C. We have used the identity $\partial_{s} *=*_{E} \partial_{s} Q^{2}$, which implies $\left\|\partial_{s} *\right\|_{s} \leq 2 \delta^{-3} c_{Q}$, and

$$
\left\|\mathrm{d}_{A^{\prime}} \varphi^{\prime}\right\|_{S}^{2}+\left\|\mathrm{d}_{A^{\prime}} \alpha^{\prime}\right\|_{S}^{2}=\left\|* \mathrm{~d}_{A^{\prime}} \alpha^{\prime}-\mathrm{d}_{A^{\prime}} \varphi^{\prime}\right\|_{S}^{2}+2 \int_{S^{3}}\left\langle\alpha^{\prime} \wedge\left[F_{A^{\prime}}, \varphi^{\prime}\right]\right\rangle
$$

which implies $\left\|\mathrm{d}_{A^{\prime}} \varphi^{\prime}\right\|_{s} \leq\left\|\mathcal{H}_{A^{\prime}} \xi^{\prime}\right\|_{s}+2\left\|F_{A^{\prime}}\right\|_{L^{\infty}\left(S^{3}\right)}^{1 / 2}\left\|\xi^{\prime}\right\|_{s}$.

Proof of Proposition 8.6 The proof of (i) is similar to that of Lemma 8.7 except for the presence of boundary terms. To control these we first use Lemma 8.7 (i) on $U:=N \backslash \partial Y$ for a neighbourhood $N \subset Y$ of $\partial Y$ on which $X_{f} \equiv 0$. It implies that $A$ and $B$ agree on $I \times U$ and hence by continuity on $I \times N$. In particular, the 1-form $\alpha(s):=B(s)-A(s) \in \Omega^{1}(Y, \mathfrak{g})$ vanishes near $\partial Y$ and hence belongs to the space $\Omega_{A(s)}^{1}(Y, \mathfrak{g})$ for every $s$. To establish unique continuation in the interior we assume, by contradiction, that $\alpha\left(s_{1}\right) \neq 0$ for some $s_{1}<s_{0}$. We will apply Theorem C.1 to $x(s)=\alpha\left(s_{1}-s\right)$ and the symmetric operator

$$
\mathbb{F}(s):=* \mathrm{~d}_{A(s)}+* \mathrm{~d} X_{f}(A(s)): \Omega_{A(s)}^{1}(Y, \mathfrak{g}) \rightarrow \Omega_{A(s)}^{1}(Y, \mathfrak{g})
$$

for $s \in I$. We have $\alpha\left(s_{0}\right)=0$ and

$$
\begin{aligned}
& \partial_{s} \alpha+\mathbb{F} \alpha=-\frac{1}{2} *[\alpha \wedge \alpha]-*\left(X_{f}(A+\alpha)-X_{f}(A)-\mathrm{d} X_{f}(A) \alpha\right), \\
& \partial_{s}\langle\alpha, \mathbb{F} \alpha\rangle-2\left\langle\partial_{s} \alpha, \mathbb{F} \alpha\right\rangle=\int_{Y}\left\langle\alpha \wedge\left[\partial_{s} A \wedge \alpha\right]\right\rangle+\int_{Y}\left\langle\alpha, \mathrm{d}^{2} X_{f}(A)\left(\partial_{s} A, \alpha\right)\right\rangle .
\end{aligned}
$$


Hence it follows from Proposition D.1 (v) that

$$
\begin{array}{r}
\left\|\partial_{s} \alpha(s)+\mathbb{F}(s) \alpha(s)\right\|_{L^{2}(Y)} \leq c_{1}\|\alpha(s)\|_{L^{2}(Y)}, \\
\partial_{s}\langle\alpha(s), \mathbb{F}(s) \alpha(s)\rangle-2\left\langle\partial_{s} \alpha(s), \mathbb{F}(s) \alpha(s)\right\rangle \leq c_{3}\|\alpha(s)\|_{L^{2}(Y)}^{2}
\end{array}
$$

for $s_{1} \leq s \leq s_{0}$ and suitable constants $c_{1}$ and $c_{3}$. This shows that the path $s \mapsto \alpha(s)$ and the operator family $\mathbb{F}(s)$ satisfy the hypotheses of Theorem C.1 with $c_{2}=0$. Hence $\alpha(s)=0$ for $s_{1}<s \leq s_{0}$ and $\alpha\left(s_{1}\right)=0$ follows by continuity, in contradiction to the assumption. The argument for $s_{1}>s_{0}$ is similar and this proves (i). Assertion (ii) follows from Lemma 8.7 (ii) and the analogous estimates for the solutions of (104). This proves the proposition.

\section{Gluing}

Let $Y$ be a compact oriented Riemannian 3-manifold with boundary $\partial Y=\Sigma$ and $\mathcal{L} \subset$ $\mathcal{A}(\Sigma)$ be a gauge invariant, monotone, irreducible Lagrangian submanifold satisfying (L1)-(L3). Fix a regular perturbation $h_{f}: \mathcal{A}(Y) \rightarrow \mathbb{R}$ in the sense of Definition 8.2.

Let $B_{0}, B_{1}, B_{2} \in \mathcal{A}(Y, \mathcal{L})$ be nondegenerate and irreducible critical points of $\mathcal{C S}_{\mathcal{L}}+h_{f}$. We denote by $\mathcal{A}\left(\mathbb{R} \times Y, \mathcal{L} ; B_{0}, B_{2}\right)$ the space of smooth connections on $\mathbb{R} \times Y$ with boundary values in $\mathcal{L}$ and $\mathcal{C}^{\infty}$-limits $B_{0}$ and $B_{2}$ as in (61); this is a special case of the notation (74). Also recall the notation $\widetilde{\mathcal{M}}\left(B_{0}, B_{1} ; X_{f}\right)$ from Section 7 for the space of solutions that are in temporal gauge over the ends, and $\mathcal{M}\left(B_{0}, B_{1} ; X_{f}\right)$ for this space modulo gauge equivalence. For $T>1$ we define a pregluing map

$$
\begin{aligned}
\widetilde{\mathcal{M}}\left(B_{0}, B_{1} ; X_{f}\right) \times \widetilde{\mathcal{M}}\left(B_{1}, B_{2} ; X_{f}\right) & \rightarrow \mathcal{A}\left(\mathbb{R} \times Y, \mathcal{L} ; B_{0}, B_{2}\right) \\
\left(\Xi_{1}, \Xi_{2}\right) & \mapsto \Xi_{1} \#_{T} \Xi_{2}
\end{aligned}
$$

as follows. The connections $\Xi_{i}=A_{i}+\Phi_{i} \mathrm{~d} s$ are in temporal gauge outside the compact set $[-1,1] \times Y$ and have limits

$$
\lim _{s \rightarrow-\infty} A_{1}(s)=B_{0}, \quad \lim _{s \rightarrow \infty} A_{1}(s)=B_{1}=\lim _{s \rightarrow-\infty} A_{2}(s), \quad \lim _{s \rightarrow \infty} A_{2}(s)=B_{2} .
$$

Define $\Xi_{1} \#_{T} \Xi_{2}:=A+\Phi \mathrm{d} s$ by

$$
\begin{aligned}
& \Phi(s):= \begin{cases}\Phi_{1}(s+T), s \leq 0, \\
\Phi_{2}(s-T), & s \geq 0,\end{cases} \\
& A(s):= \begin{cases}A_{1}\left(\frac{T}{2}-\varphi\left(-\frac{T}{2}-s\right)\right), & s<-\frac{T}{2}, \\
B_{1}, & s \in\left[-\frac{T}{2}, \frac{T}{2}\right], \\
A_{2}\left(-\frac{T}{2}+\varphi\left(-\frac{T}{2}+s\right)\right), & s>\frac{T}{2},\end{cases}
\end{aligned}
$$

Geometry $\&$ Topology, Volume 12 (2008) 
where $\varphi:(0, \infty) \rightarrow \mathbb{R}$ is a smooth function satisfying

$$
\varphi(s)=\left\{\begin{array}{ll}
s, & s \geq 2, \\
-\frac{1}{s}, & s \leq \frac{1}{2},
\end{array} \quad \partial_{s} \varphi>0 .\right.
$$

This connection is smooth because $A_{1}$ and $A_{2}$ converge exponentially as $s$ tends to $\pm \infty$. It satisfies the limit conditions and the Lagrangian boundary conditions by construction. In fact, this is why we use rescaling in time rather than convex interpolation in space. The map $\left(\Xi_{1}, \Xi_{2}\right) \mapsto \Xi_{1} \#_{T} \Xi_{2}$ is gauge equivariant in the sense that

$$
\left(u_{1}^{*} \Xi_{1}\right) \#_{T}\left(u_{2}^{*} \Xi_{2}\right)=u^{*}\left(\Xi_{1} \#_{T} \Xi_{2}\right), \quad u(s):= \begin{cases}u_{1}(s+T), & s \leq 0 \\ u_{2}(s-T), & s \geq 0\end{cases}
$$

for each pair $\left(u_{1}, u_{2}\right) \in \mathcal{G}\left(B_{0}, B_{1}\right) \times \mathcal{G}\left(B_{1}, B_{2}\right)$. Recall from the beginning of Section 7 that each $u_{1} \in \mathcal{G}\left(B_{0}, B_{1}\right)$ satisfies $\partial_{s} u_{1}(s)=0$ for $|s| \geq 1, u_{1}(s) \in \mathcal{G}_{B_{1}}$ for $s \geq 1$, and $u_{1}(s) \in \mathcal{G}_{B_{0}}$ for $s \leq-1$; similarly for $u_{2}$. Since $B_{1}$ is irreducible we have $u_{1}(s)=u_{2}(-s)=11$ for $s \geq 1$.

Theorem 9.1 Let $B_{0}, B_{1}, B_{2} \in \mathcal{A}(Y, \mathcal{L})$ be nondegenerate and irreducible critical points of $\mathcal{C} \mathcal{S}_{\mathcal{L}}+h_{f}$, and fix $\Xi_{1} \in \widetilde{\mathcal{M}}\left(B_{0}, B_{1} ; X_{f}\right)$ and $\Xi_{2} \in \widetilde{\mathcal{M}}\left(B_{1}, B_{2} ; X_{f}\right)$ with $\delta_{f}\left(\Xi_{1}\right)=\delta_{f}\left(\Xi_{2}\right)=1$. Then, for every $p>2$, there exist positive constants $\kappa, T_{0}$ and a map

$$
\tau:\left(T_{0}, \infty\right) \rightarrow \mathcal{M}^{2}\left(B_{0}, B_{2} ; X_{f}\right) / \mathbb{R}, \quad T \mapsto \tau_{T}\left(\Xi_{1}, \Xi_{2}\right)
$$

with the following properties:

(i) $\tau$ is a diffeomorphism onto its image.

(ii) The connections $\tau_{T}\left(\Xi_{1}, \Xi_{2}\right)$ converge without bubbling (as in Theorem 7.2) to the broken trajectory $\left(\Xi_{1}, \Xi_{2}\right)$ as $T \rightarrow \infty$.

(iii) If $\Xi$ is a solution of the Floer equation (15) and

$$
\left\|\Xi-\left(\Xi_{1} \#_{T} \Xi_{2}\right)\right\|_{W^{1, p}(\mathbb{R} \times Y)} \leq \kappa
$$

for some $T \geq T_{0}+1$, then its gauge and time-shift equivalence class [ $\Xi$ ] lies in the image of $\tau$.

Proof The preglued connection

$$
\Xi_{1} \#_{T} \Xi_{2}=: \Xi_{T}=A_{T}+\Phi_{T} \mathrm{~d} s
$$

is an approximate solution of the Floer equation and $\tau_{T}\left(\Xi_{1}, \Xi_{2}\right)$ will be constructed as a nearby true solution. More precisely, we have

$$
\left\|\partial_{S} A_{T}-\mathrm{d}_{A_{T}} \Phi_{T}+*\left(F_{A_{T}}+X_{f}\left(A_{T}\right)\right)\right\|_{L^{p}(\mathbb{R} \times Y)} \leq C e^{-\delta T}
$$


for some constants $C$ and $\delta>0$ by exponential decay, Theorem 5.1. We will use the inverse function theorem to find near the approximate solution $\Xi_{T}$ a true solution $\widetilde{\Xi}_{T} \in \widetilde{\mathcal{M}}\left(B_{0}, B_{2} ; X_{f}\right)$. For that purpose we use the Banach manifold structure of the space $\mathcal{A}^{1, p}\left(\mathbb{R} \times Y, \mathcal{L} ; B_{0}, B_{2}\right) ;$ see (74). Its tangent space $\mathrm{T}_{\Xi_{T}} \mathcal{A}^{1, p}\left(\mathbb{R} \times Y, \mathcal{L} ; B_{0}, B_{2}\right)$ is the space of all 1 -forms $\xi=\alpha+\varphi \mathrm{d} s$ with $\alpha \in W^{1, p}\left(\mathbb{R} \times Y, \mathrm{~T}^{*} Y \otimes \mathfrak{g}\right)$ and $\varphi \in W^{1, p}(\mathbb{R} \times Y, \mathfrak{g})$ satisfying the boundary condition $\alpha(s) \in \mathrm{T}_{A_{T}(s)} \mathcal{L}$. Using the exponential map of Theorem E. 4 and Corollary E.5 we obtain a continuously differentiable map

$$
\mathrm{T}_{\Xi_{T}} \mathcal{A}^{1, p}\left(\mathbb{R} \times Y, \mathcal{L} ; B_{0}, B_{2}\right) \supset \tilde{\mathcal{U}} \rightarrow \mathcal{A}^{1, p}\left(\mathbb{R} \times Y, \mathcal{L} ; B_{0}, B_{2}\right): \xi \mapsto \widetilde{E}\left(\Xi_{T} ; \xi\right)
$$

defined on a neighbourhood $\tilde{\mathcal{U}}$ of zero by

$$
\widetilde{E}\left(\Xi_{T} ; \xi\right):=E_{A_{T}(s)}(\alpha(s))+\left(\Phi_{T}(s)+\varphi(s)\right) \mathrm{d} s .
$$

We now look for a solution of the form $\widetilde{\Xi}_{T}=\widetilde{A}_{T}+\widetilde{\Phi}_{T} \mathrm{~d} s=\widetilde{E}\left(\Xi_{T} ; \xi\right)$, where $\xi \in \tilde{\mathcal{U}}$ satisfies ${ }^{5}$

$$
\mathrm{d}_{\Xi_{T}}^{*} \xi=0,\left.\quad * \xi\right|_{\mathbb{R} \times \partial Y}=0, \quad \xi \in \operatorname{im} \mathcal{D}_{T}^{*} .
$$

Note that $\widetilde{\Xi}_{T}$ automatically satisfies the boundary conditions $\left.\tilde{A}_{T}(s)\right|_{\partial Y} \in \mathcal{L}$ and has

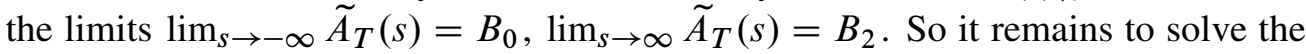
Floer equation

$$
\partial_{s} \tilde{A}_{T}-\mathrm{d}_{\tilde{A}_{T}} \tilde{\Phi}_{T}+*\left(F_{\tilde{A}_{T}}+X_{f}\left(\tilde{A}_{T}\right)\right)=0
$$

for $\xi$ subject to (109). The precise setup for the inverse function theorem is as follows: In order to keep track of the $T$-dependence we use the version [21, Proposition A.3.4.] which provides explicit constants. We apply this version of the inverse function theorem to the $\mathcal{C}^{1}$-map

$$
f_{T}: X_{T} \rightarrow Z, \quad f_{T}(\xi):=\left(F_{\widetilde{E}\left(\Xi_{T} ; \xi\right)}^{+}, \mathrm{d}_{\Xi_{T}}^{*} \xi\right) .
$$

Its domain is a neighbourhood of zero in the Banach space $X_{T}$ consisting of $\xi \in$ $\mathrm{T}_{\Xi_{T}} \mathcal{A}^{1, p}\left(\mathbb{R} \times Y, \mathcal{L} ; B_{0}, B_{2}\right)$ that satisfy the boundary condition $\left.* \xi\right|_{\mathbb{R} \times \partial Y}=0$. (Note that the domain depends on $T$. One could also work with a $T$-independent domain by using simple reparametrizations in $s \in \mathbb{R}$ to identify $X_{T} \cong X_{T_{0}}$ for a fixed $T_{0}$. This gives rise to a continuous family of inverse function problems $\widetilde{f}_{T}: X_{T_{0}} \rightarrow Z$ for

\footnotetext{
${ }^{5}$ Here $*$ denotes the Hodge $*$ operator on the four-manifold $\mathbb{R} \times Y$ unlike in (110) below. The first two conditions fix the gauge whereas the third condition fixes a complement of the kernel of the linearized operator for combined anti-self-duality and gauge fixing.
}

Geometry 8 Topology, Volume 12 (2008) 
$T \in\left[T_{0}, \infty\right)$.) The first component, $F_{\widetilde{E}}^{+}\left(\Xi_{T} ; \xi\right)$, is identified with the left hand side of (110), so the target space of $f_{T}$ is the Banach space

$$
Z=L^{p}\left(\mathbb{R} \times Y, \mathrm{~T}^{*} Y \otimes \mathfrak{g}\right) \times L^{p}(\mathbb{R} \times Y, \mathfrak{g}) .
$$

The differential $\mathrm{d} f_{T}(0)$ at $x_{0}=0$ then is the linearized operator $\mathcal{D}_{T}:=\mathcal{D}_{\Xi_{T}}$. To check that the differential $\mathrm{d} f_{T}$ is uniformly continuous at $0 \in X_{T}$ we calculate for all $\xi, \zeta \in X_{T}$

$$
\begin{aligned}
& \left\|\left(\mathrm{d} f_{T}(\xi)-\mathcal{D}_{T}\right) \zeta\right\|_{L^{p}(\mathbb{R} \times Y)}=\left\|*\left[\left(\widetilde{E}\left(\Xi_{T} ; \xi\right)-\Xi_{T}\right) \wedge * \zeta\right]\right\|_{L^{p}(\mathbb{R} \times Y)} \\
& \quad \leq C \sup _{s \in \mathbb{R}}\left\|E_{A_{T}(s)}(\alpha(s))-A_{T}(s)+\varphi(s) \mathrm{d} s\right\|_{L^{2 p}(Y)}\|\zeta\|_{W^{1, p}(\mathbb{R} \times Y)} .
\end{aligned}
$$

Here $C$ is the constant from the Sobolev embedding $W^{1, p}(Y) \hookrightarrow L^{2 p}(Y)$ and the second factor converges to zero uniformly in $T$ as $\|\xi\|_{W^{1, p}}=\|\alpha+\varphi \mathrm{d} s\|_{W^{1, p}} \rightarrow 0$. Indeed, given $\varepsilon>0$ there is $\delta_{T, s}>0$ such that $\left\|E_{A_{T}(s)}(\alpha)-A_{T}(s)\right\|_{L^{2 p}(Y)} \leq \varepsilon$ for all $\alpha \in \mathrm{T}_{A_{T}(s)} \mathcal{A}(Y, \mathcal{L})$ with $\|\alpha\|_{L^{2 p}(Y)} \leq \delta_{T, s}$. We can choose $\delta_{T, s}=\delta>0$ uniform for all $T>1, s \in \mathbb{R}$ because the image of $A_{T}$ in $\mathcal{A}(Y, \mathcal{L})$ is compact and independent of $T$.

That the linearized operator is surjective for sufficiently large $T$ with a uniform bound for its right inverse $Q_{T}:=\mathcal{D}_{T}^{*}\left(\mathcal{D}_{T} \mathcal{D}_{T}^{*}\right)^{-1}$ follows from the estimates

$$
\begin{aligned}
\|\eta\|_{W^{1, p}(\mathbb{R} \times Y)} & \leq C\left\|\mathcal{D}_{T}^{*} \eta\right\|_{L^{p}(\mathbb{R} \times Y)}, \\
\left\|\mathcal{D}_{T}^{*} \eta\right\|_{W^{1, p}(\mathbb{R} \times Y)} & \leq C\left\|\mathcal{D}_{T} \mathcal{D}_{T}^{*} \eta\right\|_{L^{p}(\mathbb{R} \times Y)} .
\end{aligned}
$$

These estimates hold for $T$ sufficiently large, and the constant $C$ is independent of $T$. The inequality (112) implies that $\mathcal{D}_{T}$ is surjective and $Q_{T}: Y \rightarrow X_{T}$ is defined, and (113) gives a uniform bound for $Q_{T}$. The proof of the estimates is as in [10, Proposition 3.9], [29, Proposition 3.9] or Theorem 7.7. It rests on the fact that the connections $\Xi_{1, T}:=\Xi_{1} \#_{T} B_{1}$ and $\Xi_{2, T}:=B_{1} \#_{T} \Xi_{2}$ (which coincide with $\Xi_{T}$ for $s \leq \frac{T}{2}$ and $s \geq-\frac{T}{2}$ respectively) satisfy exponential estimates of the form $\left\|\Xi_{i, T}-\Xi_{i}(\cdot \pm T)\right\|_{\mathcal{C}^{k}} \leq C_{k} e^{-\delta T}$, and hence their linearized operators are surjective with uniform estimates. Here we use the fact that $\Xi_{1}$ and $\Xi_{2}$ are regular in the sense of Definition 8.1.

We have thus checked that the assumptions of [21, Proposition A.3.4.] are satisfied with uniform constants for all $T \geq T_{0}$, where $T_{0}>1$ is determined by comparing (108) with [21, (A.3.5)]. Hence the inverse function theorem provides unique solutions $\xi_{T} \in \operatorname{im} Q_{T} \subset X_{T}$ of $f_{T}\left(\xi_{T}\right)=0$. In other words, we can define $\tau_{T}\left(\Xi_{1}, \Xi_{2}\right):=\widetilde{\Xi}_{T}=$ $\widetilde{E}\left(\Xi_{T}, \xi_{T}\right)$, where $\widetilde{\Xi}_{T} \in \widetilde{\mathcal{M}}\left(B_{0}, B_{2} ; X_{f}\right)$ is the unique solution of the form (109) with $\Xi_{T}=\Xi_{1} \#_{T} \Xi_{2}$. This map is gauge equivariant and induces a map to the moduli 
space. Note moreover that $\xi_{T}$ will be continuous with respect to $T$ in the $W^{1, p}$-norm and hence $\widetilde{\Xi}_{T}$ as well as $\tau$ will depend continuously on $T \in\left[T_{0}, \infty\right)$. In the following we sketch the proof of properties (i)-(iii).

The convergence in (ii) follows from the fact that the infinitesimal connection $\xi_{T}$ obtained in the inverse function theorem satisfies an estimate of the form $\left\|\xi_{T}\right\|_{W^{1, p}} \leq$ $C\left\|f_{T}(0)\right\|_{L^{p}} \leq C^{\prime} e^{-\delta T}$ for uniform constants $C, C^{\prime}$.

The index of $\tau_{T}\left(\Xi_{1}, \Xi_{2}\right)$ is given by (77), ie

$$
\begin{aligned}
\delta_{f}\left(\tau_{T}\left(\Xi_{1}, \Xi_{2}\right)\right) & =\mu_{f}\left(B_{0}, \widetilde{B}_{0}\right)-\mu_{f}\left(B_{1}, \widetilde{B}_{1}\right)+\mu_{f}\left(B_{1}, \widetilde{B}_{1}\right)-\mu_{f}\left(B_{2}, \widetilde{B}_{2}\right) \\
& =\delta_{f}\left(\Xi_{1}\right)+\delta_{f}\left(\Xi_{2}\right)=2 .
\end{aligned}
$$

Here $\widetilde{B}_{i}:[0,1] \rightarrow \mathcal{L}$ are paths from $\widetilde{B}_{i}(0)=B_{i}$ to $\widetilde{B}_{i}(1)=0$, where we pick any $\widetilde{B}_{1}$ and pick the other paths such that $\widetilde{B}_{0}$ is homotopic to the catenation of $\left.\Xi_{1}\right|_{\mathbb{R} \times \Sigma}$ with $\widetilde{B}_{1}$ and $\widetilde{B}_{1}$ is homotopic to the catenation of $\left.\Xi_{2}\right|_{\mathbb{R} \times \Sigma}$ with $\widetilde{B}_{2}$. Then, by construction, $\widetilde{B}_{0}$ is homotopic to the catenation of $\left.\left(\Xi_{1} \#_{T} \Xi_{2}\right)\right|_{\mathbb{R} \times \Sigma}$ with $\widetilde{B}_{2}$. Moreover, $\left.\tau_{T}\left(\Xi_{1}, \Xi_{2}\right)\right|_{\mathbb{R} \times \Sigma}$ is homotopic to $\left.\left(\Xi_{1} \#_{T} \Xi_{2}\right)\right|_{\mathbb{R} \times \Sigma}$.

To see that $\tau$ is a diffeomorphism note first that both domain and target are 1dimensional manifolds (by the regularity and additivity of the indices). Hence it suffices to show that $\tau$ is an injective immersion by following the argument in [10, $\mathrm{p}$ 96]. In fact, since the domain of $\tau$ is connected, it suffices to show that $\mathrm{d} \tau$ is nonzero for all sufficiently large $T$. We will show below that $\tau$ is $\mathcal{C}^{1}$-close to the pregluing $T \mapsto \Xi_{T}=\Xi_{1} \#_{T} \Xi_{2}$ as a map $\left[T_{0}, \infty\right) \rightarrow \mathcal{A}^{1, p}\left(\mathbb{R} \times Y, \mathcal{L} ; B_{0}, B_{2}\right)$, ie

$$
\left\|\frac{\mathrm{d}}{\mathrm{d} T} \widetilde{\Xi}_{T}-\frac{\mathrm{d}}{\mathrm{d} T} \Xi_{T}\right\|_{W^{1, p}(\mathbb{R} \times Y)} \underset{T \rightarrow \infty}{\longrightarrow} 0 .
$$

With this, the immersion condition $\frac{\mathrm{d}}{\mathrm{d} T} \tau \neq 0 \in \mathrm{T}_{\tau(T)} \mathcal{M}\left(B_{0}, B_{2} ; X_{f}\right) / \mathbb{R}$ follows if we can prove that the pregluing map is an immersion modulo gauge and time-shift with a uniform estimate. Indeed, taking the infimum over all $\psi \in \mathcal{C}^{\infty}(\mathbb{R} \times Y, \mathfrak{g}), \lambda \in \mathbb{R}$ we have

$$
\begin{aligned}
\inf _{\psi, \lambda}\left\|\frac{\mathrm{d}}{\mathrm{d} T} \Xi_{T}-\mathrm{d}_{\Xi_{T}} \psi-\lambda \cdot \partial_{S} \Xi_{T}\right\|_{W^{1, p}(\mathbb{R} \times Y)} & \\
\quad & \quad \inf _{\lambda}\left(\inf _{\psi}\left\|\partial_{S} A_{1}-\mathrm{d}_{A_{1}} \psi-\lambda \cdot \partial_{S} A_{1}\right\|_{W^{1, p}((-\infty,-1] \times Y)}\right. \\
& \left.\quad \quad \quad \inf _{\psi}\left\|-\partial_{S} A_{2}-\mathrm{d}_{A_{2}} \psi-\lambda \cdot \partial_{S} A_{2}\right\|_{W^{1, p}([1, \infty) \times Y)}\right) \geq \Delta>0 .
\end{aligned}
$$

Here we restricted the $W^{1, p}$-norm to the half cylinders $s \leq-T-1$ resp. $s \geq T+1$, where $\Xi_{T}(s)=A_{1}(s+T)$ resp. $\Xi_{T}(s)=A_{2}(s-T)$. We also dropped the d $s$-terms and applied various shifts. The constant $\Delta>0$ is obviously independent of $T$. It is 
positive since otherwise one could pick a minimizing sequence converging to limits $\lambda, \psi_{1}, \psi_{2}$ such that $(1-\lambda) \partial_{s} A_{1}=\mathrm{d}_{A_{1}} \psi_{1}$ and $(1+\lambda) \partial_{s} A_{2}=-\mathrm{d}_{A_{2}} \psi_{2}$. However, from unique continuation (Proposition 8.6 (ii)) we know that $\partial_{s} A_{i}(s) \notin \operatorname{imd}_{A_{i}(s)}$, so $\mathrm{d}_{A_{i}} \psi_{i}$ vanishes on both half cylinders, which leaves the contradiction $1=\lambda=-1$.

It remains to establish (114). We write $(. .$.$) for \frac{\mathrm{d}}{\mathrm{d} T}(\ldots)$ and claim that

$$
\left\|\frac{\mathrm{d}}{\mathrm{d} T} \widetilde{\Xi}_{T}-\dot{\Xi}_{T}\right\|_{W^{1, p}} \leq\left\|\partial_{1} \widetilde{E}\left(\Xi_{T}, \xi_{T}\right)-\mathrm{Id}\right\|\left\|\dot{\Xi}_{T}\right\|+\left\|\partial_{2} \tilde{E}\left(\Xi_{T}, \xi_{T}\right) \dot{\xi}_{T}\right\| \underset{T \rightarrow \infty}{\longrightarrow} 0
$$

due to the identities $\widetilde{E}(\cdot, 0)=\mathrm{Id}$ and $\partial_{2} \widetilde{E}\left(\Xi_{T}, 0\right)=\mathrm{Id}$, the boundedness of $\left\|\dot{\Xi}_{T}\right\|_{W^{1, p}}$ (due to exponential decay), and the convergence $\xi_{T} \rightarrow 0$ and $\left\|\dot{\xi}_{T}\right\|_{W^{1, p}} \rightarrow 0$. To check the latter recall the abstract setup for the inverse function theorem. Taking the $T-$ derivative of $f_{T}\left(\xi_{T}\right)=0$ we obtain

$$
\begin{aligned}
\left\|\mathrm{d} f_{T}\left(\xi_{T}\right) \dot{\xi}_{T}\right\|_{L^{p}} & =\left\|\dot{f}_{T}\left(\xi_{T}\right)\right\|_{L^{p}} \\
& =\left\|\mathrm{d}_{\widetilde{E}\left(\Xi_{T}, \xi_{T}\right)}^{+} \partial_{1} \widetilde{E}\left(\Xi_{T}, \xi_{T}\right) \dot{\Xi}_{T}\right\|_{L^{p}}+\left\|\left[\dot{\Xi}_{T} \wedge * \xi_{T}\right]\right\|_{L^{p}} \underset{T \rightarrow \infty}{\longrightarrow} 0 .
\end{aligned}
$$

This convergence uses the same estimates as before and the fact that $\mathrm{d}_{\Xi_{T}}^{+} \dot{\Xi}_{T}$ vanishes except for near $s= \pm \frac{T}{2}$, where it is exponentially small. Now write $\xi_{T}=Q_{T} \eta_{T}$ with $\eta_{T}=\mathcal{D}_{T} \xi_{T}$, then

$$
\dot{\xi}_{T}=\zeta_{T}+\dot{Q}_{T} \mathcal{D}_{T} \xi_{T} \quad \text { with } \quad \zeta_{T}=Q_{T} \dot{\eta}_{T} \in \operatorname{im} Q_{T} .
$$

We have $\left\|\dot{Q}_{T} \mathcal{D}_{T} \xi_{T}\right\|_{W^{1, p}} \rightarrow 0$ since $\xi_{T} \rightarrow 0$ and the operators $\mathcal{D}_{T}: W^{1, p} \rightarrow L^{p}$ and $\dot{Q}_{T}: L^{p} \rightarrow \operatorname{dom} \mathcal{D}_{T} \subset W^{1, p}$ are uniformly bounded. The first bound is due to $\left\|\mathcal{D}_{T}-\mathcal{D}_{T_{0}}\right\| \leq\left\|\Xi_{T}-\Xi_{T_{0}}\right\|_{\mathcal{C}^{0}} ;$ similarly $\dot{\mathcal{D}}_{T}: W^{1, p} \rightarrow L^{p}$ and $\dot{\mathcal{D}}_{T}^{*}: W^{2, p} \rightarrow W^{1, p}$ are bounded in terms of $\left\|\dot{\Xi}_{T}\right\|_{\mathcal{C}^{0}}$ resp. $\left\|\dot{\Xi}_{T}\right\|_{\mathcal{C}^{1}}$, and we have the identity $\dot{Q}_{T}=$ $\dot{\mathcal{D}}_{T}^{*}\left(\mathcal{D}_{T} \mathcal{D}_{T}^{*}\right)^{-1}-Q_{T}\left(\dot{\mathcal{D}}_{T} \mathcal{D}_{T}^{*}+\mathcal{D}_{T} \dot{\mathcal{D}}_{T}^{*}\right)\left(\mathcal{D}_{T} \mathcal{D}_{T}^{*}\right)^{-1}$. Here the uniform bound on $\left(\mathcal{D}_{T} \mathcal{D}_{T}^{*}\right)^{-1}$, that is $\|\eta\|_{W^{2, p}} \leq C\left\|\mathcal{D}_{T} \mathcal{D}_{T}^{*} \eta\right\|_{L^{p}}$, follows from combining (113) with the $W^{2, p}$-version of (112).

Finally, we can prove that $\left\|\zeta_{T}\right\|_{W^{1, p}} \rightarrow 0$ because, starting from (113),

$$
\begin{aligned}
& \left\|\zeta_{T}\right\|_{W^{1, p}} \leq C\left\|\mathcal{D}_{T} \zeta_{T}\right\|_{L^{p}} \\
& \quad \leq C\left(\left\|\left(\mathrm{~d} f_{T}\left(\xi_{T}\right)-\mathcal{D}_{T}\right) \zeta_{T}\right\|_{L^{p}}+\left\|\mathrm{d} f_{T}\left(\xi_{T}\right) \dot{\xi}_{T}\right\|_{L^{p}}+\left\|\mathrm{d} f_{T}\left(\xi_{T}\right) \dot{Q}_{T} \mathcal{D}_{T} \xi_{T}\right\|_{L^{p}}\right) .
\end{aligned}
$$

Here the first term can be absorbed into the left hand side by (111) for sufficiently large $T>T_{0}$ and the other terms converge to zero as $T \rightarrow \infty$, using a uniform bound on $\mathrm{d} f_{T}\left(\xi_{T}\right)$ from $\left\|\mathrm{d} f_{T}\left(\xi_{T}\right)-\mathcal{D}_{T}\right\| \leq\left\|\widetilde{E}\left(\Xi_{T}, \xi_{T}\right)-\Xi_{T}\right\|_{\mathcal{C}^{0}}$. This finishes the proof that $\dot{\xi}_{T} \rightarrow 0$, hence (114) holds and (i) is proven.

Assertion (iii) follows from the uniqueness statement in the inverse function theorem if we can find $u \in \mathcal{G}(\mathbb{R} \times Y), \sigma \in \mathbb{R}$, and $T^{\prime}>T_{0}$ such that $u^{*} \Xi(\cdot+\sigma)=\widetilde{E}\left(\Xi_{T^{\prime}}, \xi\right)$ 
with $\xi$ satisfying (109) and $W^{1, p}$-small. For each $\left(\sigma, T^{\prime}\right)$ close to $(0, T)$ we can use the local slice theorem to find $u_{\sigma, T^{\prime}}$ and $\xi_{\sigma, T^{\prime}}$ satisfying

$$
u_{\sigma, T^{\prime}}^{*} \Xi(\cdot+\sigma)=\widetilde{E}\left(\Xi_{T^{\prime}}, \xi_{\sigma, T^{\prime}}\right), \quad \mathrm{d}_{\Xi_{T^{\prime}}}^{*} \xi_{\sigma, T^{\prime}}=0,\left.\quad * \xi_{\sigma, T^{\prime}}\right|_{\mathbb{R} \times \partial Y}=0 .
$$

One then finds $\left(\sigma, T^{\prime}\right)$ satisfying $\xi_{\sigma, T^{\prime}} \in \operatorname{im} \mathcal{D}_{T^{\prime}}^{*}=\left(\operatorname{ker} \mathcal{D}_{T^{\prime}}\right)^{\perp}$ by a further implicit function theorem. Namely, there is a basis $\left(\eta_{1, T^{\prime}}, \eta_{2, T^{\prime}}\right)$ of $\operatorname{ker} \mathcal{D}_{T^{\prime}}$ close to $\left(\partial_{s} \Xi_{1} \#_{T^{\prime}} 0,0 \#_{T^{\prime}} \partial_{s} \Xi_{2}\right)$. Then the map $\left(\sigma, T^{\prime}\right) \mapsto\left(\left\langle\xi_{\sigma, T^{\prime}}, \eta_{1, T^{\prime}}\right\rangle,\left\langle\xi_{\sigma, T^{\prime}}, \eta_{2, T^{\prime}}\right\rangle\right)$ is invertible and has a zero close to $(0, T)$.

Remark 9.2 In Theorem 9.1 we can allow $B_{1}$ to be reducible (but still nondegenerate). Then we obtain a gluing map

$$
\tau:\left(T_{0}, \infty\right) \times\left(\mathcal{G}_{B_{1}} /\{ \pm \mathbb{1}\}\right) \rightarrow \mathcal{M}^{2+\operatorname{dim} H_{B_{1}}^{0}}\left(B_{0}, B_{2} ; X_{f}\right) / \mathbb{R}
$$

with the same properties as in Theorem 9.1. This map is constructed by starting from a preglued connection $\Xi_{1} \#_{g, T} \Xi_{2}$ that takes $g \in \mathcal{G}_{B_{1}} /\{ \pm 11\}$ into account by

$$
A(s):= \begin{cases}A_{1}\left(\frac{T}{2}-\varphi\left(-\frac{T}{2}-s\right)\right), & s \leq-\frac{T}{2}, \\ B_{1}=g^{*} B_{1}, & s \in\left[-\frac{T}{2}, \frac{T}{2}\right], \\ g^{*} A_{2}\left(-\frac{T}{2}+\varphi\left(-\frac{T}{2}+s\right)\right), & s \geq \frac{T}{2} .\end{cases}
$$

The index identity again follows from (77) and the uniformly bounded right inverse can be constructed using weighted spaces, as described in [10, 4.4.1].

This shows that the breaking of trajectories at the zero connection can be excluded in low dimensional moduli spaces since the stabilizer $\mathcal{G}_{0} \subset \mathcal{G}(Y)$ adds 3 to the index of the glued connection. However, this argument is not needed for the construction of Floer homology. In the proof of Corollary 9.3 below, we use simpler index bounds to exclude breaking at the zero connection.

Theorem 9.1 gives rise to maps

$$
\tau_{T}: \mathcal{M}^{1}\left(B_{0}, B_{1}\right) / \mathbb{R} \times \mathcal{M}^{1}\left(B_{1}, B_{2}\right) / \mathbb{R} \rightarrow \mathcal{M}^{2}\left(B_{1}, B_{2}\right) / \mathbb{R}
$$

defined by choosing one representative for each gauge and shift equivalence class in each moduli space $\mathcal{M}^{1}\left(A^{-}, A^{+}\right) / \mathbb{R}$ with $\left[A^{+}\right],\left[A^{-}\right] \in \mathcal{R}_{f} \backslash[0]$.

Corollary 9.3 Let $A^{+}, A^{-} \in \operatorname{Crit}\left(\mathcal{C S}_{\mathcal{L}}+h_{f}\right) \backslash[0]$. Then, for $T_{0}$ sufficiently large, the sets $\tau_{\left(T_{0}, \infty\right)}\left(\left[\Xi_{1}\right],\left[\Xi_{2}\right]\right) \subset \mathcal{M}^{2}\left(A^{-}, A^{+}\right) / \mathbb{R}$, indexed by $[B] \in \mathcal{R}_{f} \backslash[0]$ and pairs $\left(\left[\Xi_{1}\right],\left[\Xi_{2}\right]\right) \in \mathcal{M}^{1}\left(A^{-}, B\right) / \mathbb{R} \times \mathcal{M}^{1}\left(B, A^{+}\right) / \mathbb{R}$, are pairwise disjoint. Moreover, their complement

$$
\mathcal{M}^{2}\left(A^{-}, A^{+}\right) / \mathbb{R} \backslash \bigcup_{[0] \neq[B] \in \mathcal{R}_{f}} \bigcup_{T>T_{0}} \tau_{T}\left(\mathcal{M}^{1}\left(A^{-}, B\right) / \mathbb{R} \times \mathcal{M}^{1}\left(B, A^{+}\right) / \mathbb{R}\right)
$$


is compact.

Proof The sets $\tau_{\left(T_{0}, \infty\right)}\left(\left[\Xi_{1}\right],\left[\Xi_{2}\right]\right)$ are disjoint for $T_{0}$ sufficiently large since they converge to different broken trajectories for $T_{0} \rightarrow \infty$; see Theorem 9.1 (ii).

To prove compactness we assume by contradiction that there exists a sequence $\left[\Xi^{v}\right] \epsilon$ $\mathcal{M}^{2}\left(A^{-}, A^{+} ; X_{f}\right) / \mathbb{R}$ in the complement of the image of $\tau$ as above, and that has no convergent subsequence. These solutions have index 2 and hence fixed energy by Corollary 6.14 (i). By Theorem 7.2 we can pick a subsequence and representatives, still denoted by $\Xi^{v}$, that converge to a broken trajectory $\left(\Xi_{1}, \ldots, \Xi_{\ell}\right)$ modulo bubbling. By transversality we do not have solutions of negative index, so Corollary 7.4 implies that there is no bubbling, and the index identity in Theorem 7.2 implies $\ell \leq 2$. In the case $\ell=1$ we would obtain a convergent subsequence from Theorem 7.5, hence the limit must be a broken trajectory with two index 1 solutions and an irreducible intermediate critical point $B$. The time-shifts and gauge transformations in Theorem 7.2 can be chosen such that the limit $\left(\Xi_{1}, \Xi_{2}\right)$ consists of the fixed representatives used in the definition of $\tau_{T}$. Now the assertion of Theorem 7.7 (ii) can be reformulated as

$$
\left\|v^{v *} \Xi^{v}\left(\cdot+\frac{1}{2}\left(s_{1}^{v}+s_{2}^{\nu}\right)\right)-\Xi_{1} \#_{T^{v}} \Xi_{2}\right\|_{W^{1, p}(\mathbb{R} \times Y)} \rightarrow 0
$$

for $T^{v}:=\frac{1}{2}\left(s_{2}^{\nu}-s_{1}^{\nu}\right) \rightarrow \infty$. Then, by Theorem 9.1 (iii), $\left[\Xi^{\nu}\right]$ lies in the image of $\tau$ for sufficiently large $v$, in contradiction to the assumption.

\section{Coherent orientations}

Let $Y$ be a compact oriented Riemannian 3-manifold with boundary $\partial Y=\Sigma$ and $\mathcal{L} \subset$ $\mathcal{A}(\Sigma)$ be a gauge invariant, monotone, irreducible Lagrangian submanifold satisfying (L1)-(L3). In this section it is essential that we restrict to the case of $Y$ being connected with nonempty boundary, so that the gauge group $\mathcal{G}(Y)$ is connected. The construction of orientations for closed $Y$ can be found in [10, 5.4]. Fix a perturbation $h_{f}$ such that every critical point of $\mathcal{C} \mathcal{S}_{\mathcal{L}}+h_{f}$ is nondegenerate and every nontrivial critical point is irreducible (see Definition 8.2). For every pair of irreducible critical points $A^{-}, A^{+} \in \operatorname{Crit}\left(\mathcal{C S}_{\mathcal{L}}+h_{f}\right)$ we consider the space

$$
\mathcal{A}\left(A^{-}, A^{+}\right):=\left\{\mathbb{A} \in \mathcal{A}(\mathbb{R} \times Y, \mathcal{L})|\mathbb{A}|_{[s, s+1] \times Y} \underset{s \rightarrow \pm \infty}{\longrightarrow} 0 \mathrm{~d} s+A^{ \pm} \text {exponent. }\right\},
$$

which consists of smooth connections $\mathbb{A}=\Phi \mathrm{d} s+A$ on $\mathbb{R} \times Y$ that are given by paths $\Phi: \mathbb{R} \rightarrow \Omega^{0}(Y, \mathfrak{g})$ and $A: \mathbb{R} \rightarrow \mathcal{A}(Y, \mathcal{L})$ that converge exponentially with all derivatives to 0 and $A^{ \pm}$, respectively, as $s \rightarrow \pm \infty$. If we allow the limits $A^{ \pm}$to vary 
within gauge orbits of critical points, we obtain the spaces

$$
\mathcal{A}\left(\left[A^{-}\right],\left[A^{+}\right]\right):=\bigcup_{u^{ \pm} \in \mathcal{G}(Y)} \mathcal{A}\left(\left(u^{-}\right)^{*} A^{-},\left(u^{+}\right)^{*} A^{+}\right) .
$$

We denote by

$$
\operatorname{Or}\left(\left[A^{-}\right],\left[A^{+}\right]\right):=\bigsqcup_{\mathbb{A} \in \mathcal{A}\left(\left[A^{-}\right],\left[A^{+}\right]\right)} \operatorname{Or}\left(\mathcal{D}_{\mathbb{A}}\right) \rightarrow \mathcal{A}\left(\left[A^{-}\right],\left[A^{+}\right]\right)
$$

the principal $\mathbb{Z}_{2}$-bundle whose fibre over $\mathbb{A} \in \mathcal{A}\left(\left[A^{-}\right],\left[A^{+}\right]\right)$is the set $\operatorname{Or}\left(\mathcal{D}_{\mathbb{A}}\right)$ of orientations of the determinant line

$$
\operatorname{det}\left(\mathcal{D}_{\mathbb{A}}\right):=\Lambda^{\max }\left(\operatorname{ker} \mathcal{D}_{\mathbb{A}}\right) \otimes \Lambda^{\max }\left(\operatorname{coker} \mathcal{D}_{\mathbb{A}}\right)^{*} .
$$

Here $\mathcal{D}_{\mathbb{A}}$ is the linearized operator (31). Any homotopy $[0,1] \rightarrow \mathcal{A}\left(\left[A^{-}\right],\left[A^{+}\right]\right)$, $\lambda \mapsto A_{\lambda}$ induces an isomorphism

$$
\operatorname{Or}\left(\mathcal{D}_{\mathbb{A}_{0}}\right) \rightarrow \operatorname{Or}\left(\mathcal{D}_{\mathbb{A}_{1}}\right)
$$

by path lifting. A gauge transformation $u \in \mathcal{G}(\mathbb{R} \times Y)$ which converges exponentially to $u^{ \pm} \in \mathcal{G}(Y)$ as $s \rightarrow \pm \infty$ gives rise to a bundle isomorphism

$$
u^{*}: \operatorname{Or}\left(A^{-}, A^{+}\right) \rightarrow \operatorname{Or}\left(\left(u^{-}\right)^{*} A^{-},\left(u^{+}\right)^{*} A^{+}\right)
$$

induced by the conjugate action of $u$ on kernel and cokernel. The pregluing construction in (107) for $\mathbb{A}_{1} \in \mathcal{A}\left(B_{0}, B_{1}\right)$ and $\mathbb{A}_{2} \in \mathcal{A}\left(B_{1}, B_{2}\right)$ induces a natural isomorphism

$$
\sigma_{T}: \operatorname{Or}\left(\mathcal{D}_{\mathbb{A}_{1}}\right) \otimes \operatorname{Or}\left(\mathcal{D}_{\mathbb{A}_{2}}\right) \rightarrow \operatorname{Or}\left(\mathcal{D}_{\mathbb{A}_{1} \#_{T} \mathbb{A}_{2}}\right)
$$

for sufficiently large $T$. If both $\mathcal{D}_{\mathbb{A}_{1}}$ and $\mathcal{D}_{\mathbb{A}_{2}}$ are surjective, then $\mathcal{D}_{\mathbb{A}_{1} \#_{T} \mathbb{A}_{2}}$ is surjective for $T$ sufficiently large, by estimates as in the proof of Theorem 7.7, and $\sigma_{T}$ is induced by the isomorphism $\operatorname{ker}\left(\mathcal{D}_{\mathbb{A}_{1}}\right) \times \operatorname{ker}\left(\mathcal{D}_{\mathbb{A}_{2}}\right) \rightarrow \operatorname{ker}\left(\mathcal{D}_{\mathbb{A}_{1} \#_{T} \mathbb{A}_{2}}\right)$. The general case is reduced to the surjective case by the method of stabilizations as in $[9$, Section 3(a)].

We will also have to glue connections over $S^{4}$ to connections over $\mathbb{R} \times Y$. For that purpose we denote by $\mathcal{A}\left(P_{u}\right)$ the space of connections on the bundle $P_{u}$ that is obtained by gluing two copies of $\mathbb{C}^{2} \times B^{4}$ with the transition function $u \in \mathcal{G}\left(S^{3}\right)$. Then for every $\mathbb{A} \in \mathcal{A}\left(A^{-}, A^{+}\right)$and $\Xi_{u} \in \mathcal{A}\left(P_{u}\right)$ we can construct a preglued connection $\mathbb{A}_{T} \Xi_{u} \in \mathcal{A}\left(A^{-}, \bar{u}^{*} A^{+}\right)$by taking the connected sum $(\mathbb{R} \times Y) \#_{\partial D_{T}} S^{4}$ and trivializing the induced bundle over $\mathbb{R} \times Y$. Here we denote by $D_{T} \subset \mathbb{R} \times Y$ the ball of radius $T^{-1}$ centred at $(0, y)$ for some $y \in \operatorname{int}(Y)$, and after the trivialization we have

$$
\left.\left(\mathbb{A}_{T} \Xi_{u}\right)\right|_{(\mathbb{R} \times Y) \backslash D_{T}}=\tilde{u}^{*} \mathbb{A}
$$


for a gauge transformation $\tilde{u}$ on $(\mathbb{R} \times Y) \backslash D_{T}$ with $\left.\tilde{u}\right|_{\partial D_{T}} \cong u$. We fix these extensions such that $\left.\tilde{u}\right|_{(-\infty,-1] \times Y} \equiv \mathbb{1 l}$ and $\left.\tilde{u}\right|_{[1, \infty) \times Y} \equiv \mathbb{1}$, and hence $\left.\tilde{u}\right|_{\mathbb{R} \times \partial Y}$ defines a path $\bar{u}: \mathbb{R} \rightarrow \mathcal{G}(\Sigma)$ with $\bar{u}(s)=\mathbb{1}$ for $|s| \geq 1$. A partial integration on $[-1,1] \times Y$ then shows that the degree of this loop is $\operatorname{deg}(\bar{u})=\operatorname{deg}(u)$. So we have both $\mathbb{A}, \mathbb{A}_{T} \Xi_{u} \in$ $\mathcal{A}\left(A^{-}, A^{+}\right)$, but the homotopy classes (of paths in $\mathcal{L}$ with fixed endpoints) of $\left.\mathbb{A}\right|_{\partial Y}$ and $\left.\left(\mathbb{A}_{T} \Xi_{u}\right)\right|_{\partial Y}$ differ by $\operatorname{deg}(u)$. The determinant line bundle over the contractible space $\mathcal{A}\left(P_{u}\right)$ is canonically oriented (compatible with gauge transformations, homotopies, and gluing; see eg [11, Proposition 5.4.1]), and as before pregluing induces an isomorphism

$$
\sigma_{T}: \operatorname{Or}\left(\mathcal{D}_{\mathbb{A}}\right) \otimes \operatorname{Or}\left(\mathcal{D}_{\Xi_{u}}\right) \rightarrow \operatorname{Or}\left(\mathcal{D}_{\mathbb{A}_{T} \Xi_{u}}\right)
$$

for $T$ sufficiently large. The various isomorphisms, induced by homotopies, gauge transformations, and pregluing, all commute in the appropriate sense.

Definition 10.1 A system of coherent orientations is a collection of sections

$$
\mathcal{A}\left(\left[A^{-}\right],\left[A^{+}\right]\right) \rightarrow \operatorname{Or}\left(\left[A^{-}\right],\left[A^{+}\right]\right): \mathbb{A} \mapsto o_{\mathbb{A}},
$$

one for each pair $\left[A^{-}\right],\left[A^{+}\right] \in \operatorname{Crit}\left(\mathcal{C S}_{\mathcal{L}}+h_{f}\right) / \mathcal{G}(Y) \backslash[0]$ of nontrivial gauge equivalence classes of critical points, satisfying the following conditions.

(Homotopy) The sections $o: \mathcal{A}\left(\left[A^{-}\right],\left[A^{+}\right]\right) \rightarrow \operatorname{Or}\left(\left[A^{-}\right],\left[A^{+}\right]\right)$are continuous. In other words, if $[0,1] \rightarrow \mathcal{A}\left(\left[A^{-}\right],\left[A^{+}\right]\right): \lambda \mapsto \mathbb{A}_{\lambda}$ is a continuous path, then the induced isomorphism $\operatorname{Or}\left(\mathcal{D}_{\mathbb{A}_{0}}\right) \rightarrow \operatorname{Or}\left(\mathcal{D}_{\mathbb{A}_{1}}\right)$ sends $o_{\mathbb{A}_{0}}$ to $o_{\mathbb{A}_{1}}$.

(Equivariance) For every $\mathbb{A} \in \mathcal{A}\left(A^{-}, A^{+}\right)$and every $u \in \mathcal{G}(\mathbb{R} \times Y)$ that converges exponentially to $u^{ \pm} \in \mathcal{G}(Y)$ as $s \rightarrow \pm \infty$ we have

$$
o_{u * \mathbb{A}}=u^{*} o_{\mathbb{A}} .
$$

(Catenation) Let $\mathbb{A} \in \mathcal{A}\left(B_{0}, B_{1}\right)$ and $\mathbb{A}^{\prime} \in \mathcal{A}\left(B_{1}, B_{2}\right)$, then for $T$ sufficiently large we have

$$
o_{\mathbb{A} \#_{T} \mathbb{A}^{\prime}}=\sigma_{T}\left(o_{\mathbb{A}} \otimes o_{\mathbb{A}^{\prime}}\right) .
$$

(Sum) Let $\mathbb{A} \in \mathcal{A}\left(A^{-}, A^{+}\right), u \in \mathcal{G}\left(S^{3}\right)$, and $\Xi_{u} \in \mathcal{A}\left(P_{u}\right)$, then for $T$ sufficiently large we have

$$
o_{\mathbb{A} \#_{T} \Xi_{u}}=\sigma_{T}\left(o_{\mathbb{A}} \otimes o_{\Xi_{u}}\right) .
$$

(Constant) If $\mathbb{A} \equiv A^{-}=A^{+}$, then $o_{\mathbb{A}}$ is the orientation induced by the canonical isomorphism $\operatorname{det}\left(\mathcal{D}_{\mathbb{A}}\right) \rightarrow \mathbb{R}$. (Under this assumption $\mathcal{D}_{\mathbb{A}}$ is bijective.)

Remark 10.2 (i) The (Equivariance) axiom follows from the (Homotopy) axiom. To see this note that, since $Y$ is connected with nonempty boundary, the gauge groups $\mathcal{G}(Y)$ and hence $\mathcal{G}(\mathbb{R} \times Y)$ are connected. (Here we do not fix the boundary values 
or limits of the gauge transformations.) The claim then follows from the following observation.

(ii) For every continuous path $[0,1] \rightarrow \mathcal{G}(\mathbb{R} \times Y): \lambda \mapsto u_{\lambda}$ with $u_{0}=\mathbb{1}$ the isomorphism $u_{1}^{*}: \operatorname{det}\left(\mathcal{D}_{\mathbb{A}}\right) \rightarrow \operatorname{det}\left(\mathcal{D}_{u_{1}^{*} \mathbb{A}}\right)$ coincides with the isomorphism induced by the homotopy $\lambda \mapsto u_{\lambda}^{*} \mathbb{A}$. To see this consider the continuous family of paths $[0,1] \rightarrow \mathcal{G}(\mathbb{R} \times Y)$ : $\lambda \mapsto u_{\tau \lambda}$ for $\tau \in[0,1]$. Then the assertion holds obviously for $\tau=0$ (both maps are the identity) and hence, by continuity, for all $\tau$.

Theorem 10.3 Fix representatives $B_{1}, \ldots, B_{N}$, one for each nontrivial gauge equivalence class in $\operatorname{Crit}\left(\mathcal{C S}_{\mathcal{L}}+h_{f}\right) / \mathcal{G}(Y) \backslash[0]$, connections $\mathbb{A}_{i} \in \mathcal{A}\left(B_{i}, B_{i+1}\right)$, and orientations $o_{i} \in \operatorname{Or}\left(\mathcal{D}_{\mathbb{A}_{i}}\right)$ for $i=1, \ldots, N-1$. Then there is a unique system of coherent orientations $o_{\mathbb{A}} \in \operatorname{Or}\left(\mathcal{D}_{\mathbb{A}}\right)$ such that $o_{\mathbb{A}_{i}}=o_{i}$ for all $i$.

The proof of this theorem will make use of the following lemma.

Lemma 10.4 Fix a pair $A^{ \pm} \in \mathcal{A}(Y, \mathcal{L})$ of irreducible and nondegenerate critical points of $\mathcal{C} \mathcal{S}_{\mathcal{L}}+h_{f}$. Let $[0,1] \rightarrow \mathcal{A}\left(\left[A^{-}\right],\left[A^{+}\right]\right): \lambda \mapsto \mathbb{A}_{\lambda}$ be a smooth path and $u \in \mathcal{G}(\mathbb{R} \times Y)$ such that $\mathbb{A}_{1}=u^{*} \mathbb{A}_{0}$. Then the isomorphism

$$
u^{*}: \operatorname{Or}\left(\mathcal{D}_{\mathbb{A}_{0}}\right) \rightarrow \operatorname{Or}\left(\mathcal{D}_{\mathbb{A}_{1}}\right)
$$

agrees with the one induced by the path $\lambda \mapsto \mathbb{A}_{\lambda}$. In particular, the orientation bundle $\operatorname{Or}\left(\left[A^{-}\right],\left[A^{+}\right]\right) \rightarrow \mathcal{A}\left(\left[A^{-}\right],\left[A^{+}\right]\right)$admits a trivialization.

Proof By continuity, it suffices to prove the identity under the assumption $\partial_{s} A_{\lambda}(s)=0$, $\Phi_{\lambda}(s)=0$, and $\partial_{s} u(s)=0$ for $|s| \geq 1$. Then there are paths $[0,1] \rightarrow \mathcal{G}(Y), \lambda \mapsto v_{\lambda}^{ \pm}$ such that $\left(v_{\lambda}^{-}\right)^{*} A_{\lambda}(s)=A^{-}$for $s \leq-1$ and $\left(v_{\lambda}^{+}\right)^{*} A_{\lambda}(s)=A^{+}$for $s \geq 1$. We can replace $A^{ \pm}$by $\left(\left(v_{0}^{ \pm}\right)^{-1}\right)^{*} A^{ \pm}$and thus assume in addition that $v_{0}^{ \pm}=11$. Now there is a smooth map $[0,1] \times \mathbb{R} \rightarrow \mathcal{G}(Y):(\lambda, s) \mapsto u_{\lambda}(s)$ such that $u_{0} \equiv \mathbb{1}, u_{\lambda}(s)=v_{\lambda}^{-}$for $s \leq-1$ and $u_{\lambda}(s)=v_{\lambda}^{+}$for $s \geq 1$. Define

$$
\mathbb{A}_{\lambda}^{\tau}:=u_{\lambda \tau}^{*} \mathbb{A}_{\lambda}, \quad u^{\tau}:=u_{0}^{-1} u u_{\tau}
$$

for every $\tau \in[0,1]$. Then we have $\mathbb{A}_{1}^{\tau}=\left(u^{\tau}\right)^{*} \mathbb{A}_{0}^{\tau}$. By continuity, the assertion now holds for $\tau=1$ if and only if it holds for $\tau=0$, that is for the original pair $\left(\left\{\mathbb{A}_{\lambda}\right\}, u\right)$. For $\tau=1$ we have $A_{\lambda}^{1}(s)=A^{ \pm}$and $u^{1}(s)=1$ for $\pm s \geq 1$. 
Finally, we prove the lemma in the case $A_{\lambda}(s)=A^{ \pm}$for $\pm s \geq 1$. For $T \geq 2$ we define the catenation $\mathbb{B}_{\lambda}^{T}:=\Phi_{\lambda}^{T} \mathrm{~d} s+B_{\lambda}^{T} \in \mathcal{A}(\mathbb{R} / 2 T \mathbb{Z} \times Y, \mathcal{L})$ and $u^{T} \in \mathcal{G}(\mathbb{R} / 2 T \mathbb{Z} \times Y)$ by

$$
\begin{aligned}
B_{\lambda}^{T}(s) & := \begin{cases}A_{\lambda}(s), & -T / 2 \leq s \leq T / 2, \\
A_{0}(T-s), & T / 2 \leq s \leq 3 T / 2,\end{cases} \\
\Phi_{\lambda}^{T}(s): & = \begin{cases}\Phi_{\lambda}(s), & -T / 2 \leq s \leq T / 2, \\
\Phi_{0}(T-s), & T / 2 \leq s \leq 3 T / 2,\end{cases} \\
u^{T}(s): & = \begin{cases}u(s), & -T / 2 \leq s \leq T / 2, \\
1, & T / 2 \leq s \leq 3 T / 2 .\end{cases}
\end{aligned}
$$

Then $B_{\lambda}^{T}(s)=A^{ \pm}$and $\Phi_{\lambda}^{T}=0$ for $\pm s \in[1, T-1]$. Moreover we have $\mathbb{B}_{1}^{T}=\left(u^{T}\right)^{*} \mathbb{B}_{0}^{T}$. For $T$ sufficiently large the linear gluing theory gives rise to a continuous family of isomorphisms

$$
\varphi_{\lambda}^{T}: \operatorname{Or}\left(\mathcal{D}_{\mathbb{A}_{\lambda}}\right) \rightarrow \operatorname{Or}\left(\mathcal{D}_{\mathbb{B}_{\lambda}^{T}}\right),
$$

where $\mathcal{D}_{\mathbb{B}_{\lambda}^{T}}$ denotes the anti-self-duality operator on $\mathbb{R} / 2 T \mathbb{Z} \times Y$ introduced in Section 4. The gluing operators commute with the gauge transformations, ie

$$
\varphi_{1}^{T} \circ u^{*}=\left(u^{T}\right)^{*} \circ \varphi_{0}^{T}: \operatorname{Or}\left(\mathcal{D}_{\mathbb{A}_{0}}\right) \rightarrow \operatorname{Or}\left(\mathcal{D}_{\mathbb{B}_{1}^{T}}\right) .
$$

The isomorphisms induced by the homotopies $\lambda \mapsto \mathbb{A}_{\lambda}$ and $\lambda \mapsto \mathbb{B}_{\lambda}^{T}$ satisfy the same relation. By Theorem 4.3 (iv) (with $v=\mathbb{1}$ ), the isomorphism $\left(u^{T}\right)^{*}: \operatorname{Or}\left(\mathcal{D}_{\mathbb{B}_{0}^{T}}\right) \rightarrow$ $\operatorname{Or}\left(\mathcal{D}_{\mathbb{B}_{1}^{T}}\right)$ agrees with the one induced by the path $\lambda \mapsto B_{\lambda}^{T}$. Hence the same holds for $u^{*}$ and this proves the desired identity.

To see that $\operatorname{Or}\left(\left[A^{-}\right],\left[A^{+}\right]\right) \rightarrow \mathcal{A}\left(\left[A^{-}\right],\left[A^{+}\right]\right)$admits a trivialization we only need to check that parallel transport around loops induces the identity isomorphism on the fibre This follows immediately from the identification of the homotopy induced isomorphism with $u^{*}: \operatorname{Or}\left(\mathcal{D}_{\mathbb{A}_{0}}\right) \rightarrow \operatorname{Or}\left(\mathcal{D}_{\mathbb{A}_{0}}\right)$ for $u=11$.

Proof of Theorem 10.3 By the (Homotopy) and (Constant) axioms, the orientation bundle over the constant component of $\mathcal{A}\left(\left[B_{i}\right],\left[B_{i}\right]\right)$ is canonically oriented. The orientation on the other components of $\mathcal{A}\left(\left[B_{i}\right],\left[B_{i}\right]\right)$ is determined by the (Sum) axiom because any connection $\mathbb{A} \in \mathcal{A}\left(\left[B_{i}\right],\left[B_{i}\right]\right)$ is homotopic to $\mathbb{B}_{i} \#_{T} \Xi_{u}$ for the constant solution $\mathbb{B}_{i} \equiv B_{i}$, a connection $\Xi_{u}$ over $S^{4}$ associated to a nontrivial $u \in \mathcal{G}\left(S^{3}\right)$, and any $T>0$. Indeed, since $\mathcal{G}(Y)$ is connected, $\mathbb{A}$ can be homotoped to a connection with fixed limits in $\mathcal{A}\left(B_{i}, B_{i}\right)$. Moreover, there is a homotopy equivalence $\mathcal{A}\left(B_{i}, B_{i}\right) \rightarrow$ $\mathcal{C}^{\infty}\left(S^{1}, \mathcal{L}\right)$ which assigns to each connection $\mathbb{A} \in \mathcal{A}\left(B_{i}, B_{i}\right)$ a based loop in $\mathcal{L}$ obtained from the path $\left.\mathbb{A}\right|_{\partial Y}: \mathbb{R} \rightarrow \mathcal{L}$ with endpoints $\left.B_{i}\right|_{\partial Y}$. Now, by (L2), the loop $\left.\mathbb{A}\right|_{\partial Y}$ in $\mathcal{L}$ is homotopic to $\left.\widehat{u}^{*} B_{1}\right|_{\partial Y}$ for some loop $\widehat{u}: S^{1} \rightarrow \mathcal{G}(\Sigma)$. Hence $\mathbb{A}$ is homotopic to $\mathbb{B}_{i} \#_{T} \Xi_{u}$ for the associated $u \in \mathcal{G}\left(S^{3}\right)$. Similarly, the orientation bundle over 
$\mathcal{A}\left(\left[B_{i}\right],\left[B_{i+1}\right]\right)$ is oriented by $o_{\mathbb{A}_{i}}$ and the (Homotopy) and (Sum) axioms, because any connection in $\mathcal{A}\left(\left[B_{i}\right],\left[B_{i+1}\right]\right)$ is homotopic to $\mathbb{A}_{i} \#_{T} \Xi_{u}$ for some $u \in \mathcal{G}\left(S^{3}\right)$. Finally, the orientation bundles over general spaces $\mathcal{A}\left(\left[B_{i}\right],\left[B_{j}\right]\right)$ are oriented by the (Catenation) axiom and the previously fixed orientations. This proves uniqueness.

To establish existence note that, by Lemma 10.4, we have a choice of two possible orientations over every component of each $\mathcal{A}\left(\left[A^{-}\right],\left[A^{+}\right]\right)$. Each of the possible combinations of choices satisfies the (Homotopy) axiom by construction. To see that the choices can be made such that the (Constant), (Catenation), and (Sum) axioms are satisfied (and so the (Equivariance) axiom follows from Remark 10.2), one needs to check that the isomorphisms in the (Catenation), (Sum), and (Homotopy) axioms all commute. For example, let $\mathbb{A}_{\lambda} \in \mathcal{A}\left(B_{i}, B_{j}\right)$ and $\mathbb{A}_{\lambda}^{\prime} \in \mathcal{A}\left(B_{j}, B_{k}\right)$ be smooth families parametrized by $\lambda \in[0,1]$ and denote by

$$
\begin{gathered}
\rho: \operatorname{Or}\left(\mathcal{D}_{\mathbb{A}_{0}}\right) \rightarrow \operatorname{Or}\left(\mathcal{D}_{\mathbb{A}_{1}}\right), \quad \rho^{\prime}: \operatorname{Or}\left(\mathcal{D}_{\mathbb{A}_{0}^{\prime}}\right) \rightarrow \operatorname{Or}\left(\mathcal{D}_{\mathbb{A}_{1}^{\prime}}\right), \\
\rho^{T}: \operatorname{Or}\left(\mathcal{D}_{\mathbb{A}_{0} \#_{T} \mathbb{A}_{0}^{\prime}}\right) \rightarrow \operatorname{Or}\left(\mathcal{D}_{\mathbb{A}_{1} \#_{T} \mathbb{A}_{1}^{\prime}}\right)
\end{gathered}
$$

the isomorphisms induced by the homotopies $\lambda \mapsto \mathbb{A}_{\lambda}, \mathbb{A}_{\lambda}^{\prime}$, and $\mathbb{A}_{\lambda} \# \mathbb{A}_{\lambda}^{\prime}$. Let

$$
\sigma_{\lambda}^{T}: \operatorname{Or}\left(\mathcal{D}_{\mathbb{A}_{\lambda}}\right) \otimes \operatorname{Or}\left(\mathcal{D}_{\mathbb{A}_{\lambda}^{\prime}}\right) \rightarrow \operatorname{Or}\left(\mathcal{D}_{\mathbb{A}_{\lambda} \#_{T} \mathbb{A}_{\lambda}^{\prime}}\right)
$$

denote the catenation isomorphisms for $T$ sufficiently large. A parametrized version of the linear gluing construction then proves that

$$
\sigma_{1}^{T} \circ\left(\rho \otimes \rho^{\prime}\right)=\rho^{T} \circ \sigma_{0}^{T} .
$$

A similar statement holds for the (Homotopy) and (Sum) isomorphisms. That two (Catenation) isomorphisms commute is a kind of associativity rule modulo homotopy and the proof involves a simultaneous gluing construction for three connecting trajectories; similarly for the commutation rules of the (Sum) and (Catenation) isomorphisms. All these arguments are exactly as in the standard theory and the details will be omitted. $\square$

\section{Floer homology}

Let $Y$ be a compact connected oriented 3-manifold with boundary $\partial Y=\Sigma$ and $\mathcal{L} \subset$ $\mathcal{A}(\Sigma)$ be a gauge invariant, monotone, irreducible Lagrangian submanifold satisfying (L1)-(L3). Fix a Riemannian metric $g$ on $Y$, a regular perturbation $(\gamma, f) \in \Gamma_{m} \times \mathcal{F}_{m}$ as in Theorem 8.4, and a system $\mathfrak{o}=\left\{o_{\mathbb{A}}\right\}_{\mathbb{A}}$ of coherent orientations as in Theorem 10.3. Associated to these data we define a Floer homology group $\operatorname{HF}(Y, \mathcal{L} ; g, f, \mathfrak{o})$ as follows. 
Since the trivial connection is nondegenerate by (L3), the set

$$
\mathcal{R}_{f}:=\left\{A \in \mathcal{A}(Y)\left|F_{A}+X_{f}(A)=0, A\right|_{\partial Y} \in \mathcal{L}\right\} / \mathcal{G}(Y)
$$

of gauge equivalence classes of critical points of $\mathcal{C S}_{\mathcal{L}}+h_{f}$ is finite, by Proposition 3.7. The nontrivial critical points determine a chain complex

$$
\mathrm{CF}(Y, \mathcal{L} ; f):=\bigoplus_{[A] \in \mathcal{R}_{f} \backslash[0]} \mathbb{Z}\langle A\rangle
$$

with a $\mathbb{Z} / 8 \mathbb{Z}$-grading $\mu_{f}: \mathcal{R}_{f} \rightarrow \mathbb{Z} / 8 \mathbb{Z}$ defined by the spectral flow (see Corollary 6.12). We emphasize that the spectral flow is invariant under homotopies of the metric and of the perturbation with fixed critical points. To define the boundary operator we consider the space

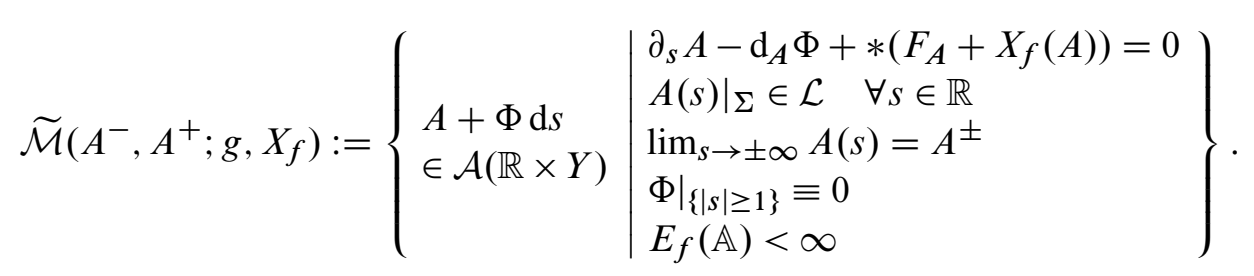

This space is invariant under the group $\mathcal{G}\left(A^{-}, A^{+}\right)$of gauge transformations $u \in$ $\mathcal{G}(\mathbb{R} \times Y)$ that satisfy $u(s)=u^{ \pm} \in \mathcal{G}_{A^{ \pm}}$for $\pm s \geq 1$. The quotient spaces

$$
\widetilde{\mathcal{M}}\left(A^{-}, A^{+} ; g, X_{f}\right) / \mathcal{G}\left(A^{+}, A^{-}\right)
$$

are canonically isomorphic for different choices of representatives $A^{ \pm}$of critical points. The index of the linearized operator at $[\mathbb{A}]$ is $\delta_{f}(\mathbb{A}) \equiv \mu_{f}\left(A^{-}\right)-\mu_{f}\left(A^{+}\right)$(modulo $8)$. For $k \in \mathbb{Z}$ we denote the index $k$ part of the Floer moduli space by

$$
\mathcal{M}^{k}\left(A^{-}, A^{+} ; g, X_{f}\right):=\left\{[\mathbb{A}] \in \widetilde{\mathcal{M}}\left(A^{-}, A^{+} ; g, X_{f}\right) / \mathcal{G}\left(A^{-}, A^{+}\right) \mid \delta_{f}(\mathbb{A})=k\right\} .
$$

For $k \leq 7$ this is a smooth $k$-dimensional manifold (see Section 6 and Definition 8.2). The energy of a solution in this space is $E_{f}(\mathbb{A})=\frac{1}{2} \pi^{2}\left(k+\eta_{f}\left(A^{+}\right)-\eta_{f}\left(A^{-}\right)\right)$ by Corollary 6.14 (i), and hence is independent of $\mathbb{A}$. Furthermore, $\mathbb{R}$ acts on $\mathcal{M}^{k}\left(A^{-}, A^{+} ; g, X_{f}\right)$ by time-shift, and the action is proper and free unless $A^{-}=A^{+}$ and $k=0$. For $k=1$ the quotient space $\mathcal{M}^{1}\left(A^{-}, A^{+} ; X_{f}\right) / \mathbb{R}$ is a finite set, by Corollary 7.6. Counting the elements with signs gives rise to a boundary operator on $\mathrm{CF}(Y, \mathcal{L} ; f)$ via

$$
\partial\left\langle A^{-}\right\rangle:=\sum_{\left[A^{+}\right] \in \mathcal{R}_{f} \backslash[0]}\left(\sum_{[\mathbb{A}] \in \mathcal{M}^{1}\left(A^{-}, A^{+} ; g, X_{f}\right) / \mathbb{R}} v(\mathbb{A})\right)\left\langle A^{+}\right\rangle .
$$


Here $v(\mathbb{A}):=1$ whenever the element $\partial_{s} \mathbb{A} \in \operatorname{ker} \mathcal{D}_{\mathbb{A}}=\operatorname{det}\left(\mathcal{D}_{\mathbb{A}}\right)$ is positively oriented with respect to $o_{\mathbb{A}}$ and $v(\mathbb{A}):=-1$ otherwise. The next two theorems are the main results of this paper; their proofs take up the rest of this section.

Theorem 11.1 The operator $\partial: \mathrm{CF}(Y, \mathcal{L} ; f) \rightarrow \mathrm{CF}(Y, \mathcal{L}, f)$ defined by (115) satisfies $\partial \circ \partial=0$.

The Floer homology group of the pair $(Y, \mathcal{L})$ equipped with the regular data $(g, f, \mathfrak{o})$ is defined by

$$
\operatorname{HF}(Y, \mathcal{L} ; g, f, \mathfrak{o}):=\frac{\operatorname{ker} \partial: \mathrm{CF}(Y, \mathcal{L} ; f) \rightarrow \mathrm{CF}(Y, \mathcal{L} ; f)}{\operatorname{im} \partial: \operatorname{CF}(Y, \mathcal{L} ; f) \rightarrow \operatorname{CF}(Y, \mathcal{L} ; f)} .
$$

The next theorem shows that it is independent of the choices of metric, perturbation, and coherent orientations.

Theorem 11.2 There is a collection of isomorphisms

$$
\Phi^{\beta \alpha}: \operatorname{HF}\left(Y, \mathcal{L} ; g^{\alpha}, f^{\alpha}, \mathfrak{o}^{\alpha}\right) \rightarrow \operatorname{HF}\left(Y, \mathcal{L} ; g^{\beta}, f^{\beta}, \mathfrak{o}^{\beta}\right),
$$

one for any two regular triples $\left(g^{\alpha}, f^{\alpha}, \mathfrak{o}^{\alpha}\right)$ and $\left(g^{\beta}, f^{\beta}, \mathfrak{o}^{\beta}\right)$, such that

$$
\Phi^{\gamma \beta} \circ \Phi^{\beta \alpha}=\Phi^{\gamma \alpha}, \quad \Phi^{\alpha \alpha}=\mathrm{Id}
$$

for any three regular triples $\left(g^{\alpha}, f^{\alpha}, \mathfrak{o}^{\alpha}\right),\left(g^{\beta}, f^{\beta}, \mathfrak{o}^{\beta}\right)$, and $\left(g^{\gamma}, f^{\gamma}, \mathfrak{o}^{\gamma}\right)$.

Proof of Theorem 11.1 For $A^{ \pm} \in \mathcal{R}_{f} \backslash[0]$ denote

$$
n\left(A^{-}, A^{+}\right):=\sum_{[\mathbb{A}] \in \mathcal{M}^{1}\left(A^{-}, A^{+} ; g, X_{f}\right) / \mathbb{R}} v(\mathbb{A}) .
$$

Then the equation $\partial \circ \partial=0$ is equivalent to the formula

$$
\sum_{[B] \in \mathcal{R}_{f} \backslash[0]} n\left(A^{-}, B\right) n\left(B, A^{+}\right)=0
$$

for all $A^{ \pm} \in \mathcal{R}_{f} \backslash[0]$. The proof of (117) is exactly as in the standard case. One studies the moduli space $\mathcal{M}^{2}\left(A^{-}, A^{+}\right) / \mathbb{R}$. This is a 1 -manifold, oriented by the coherent orientations of Theorem 10.3. By Corollary 9.3 its ends are in one-to-one correspondence with pairs of trajectories in $\mathcal{M}^{1}\left(A^{-}, B ; X_{f}\right) / \mathbb{R} \times \mathcal{M}^{1}\left(B, A^{+} ; X_{f}\right) / \mathbb{R}$ for any critical point $[B] \in \mathcal{R}_{f} \backslash[0]$, which are exactly what is counted on the left hand side of (117). By the (Catenation) axiom in Section 10 the signs agree with the orientation of the boundary of $\mathcal{M}^{2}\left(A^{-}, A^{+} ; X_{f}\right) / \mathbb{R}$. Hence the sum must be zero and this proves $\partial \circ \partial=0$. 
Proof of Theorem 11.2 That the Floer homology groups are independent of the choice of the system of coherent orientations is obvious; two such systems give rise to isomorphic boundary operators via a sign change isomorphism (with \pm 1 on the diagonal). To prove the independence of metric and perturbation, we fix two Riemannian metrics $g^{ \pm}$on $Y$ and two sets of regular perturbation data $\left(\gamma^{ \pm}, f^{ \pm}\right)$. We will construct a chain map from $\mathrm{CF}\left(Y, \mathcal{L} ; g^{-}, f^{-}\right)$to $\mathrm{CF}\left(Y, \mathcal{L} ; g^{+}, f^{+}\right)$following the familiar pattern. As in the closed case we choose a metric $\widetilde{g}$ on $\mathbb{R} \times Y$ such that $\tilde{g}=g^{ \pm}$for $\pm s$ sufficiently large. However, unlike the closed case this metric cannot necessarily be chosen in split form since it is required to be compatible with the boundary space-time splitting in the sense of Definition 6.2 (see Example 6.4 or [35, Example 1.4]). Next we choose a holonomy perturbation $\tilde{X}: \mathcal{A}(\mathbb{R} \times Y) \rightarrow \Omega^{2}(\mathbb{R} \times Y, \mathfrak{g})$ of the form $\tilde{X}=\beta X_{f^{-}}+(1-\beta) X_{f^{+}}+X_{f^{\prime}}$ for some cutoff function $\beta \in \mathcal{C}^{\infty}(\mathbb{R},[0,1])$ and a further holonomy perturbation $X_{f^{\prime}}$ as in Definition 6.6. This uses thickened loops $\gamma_{i}^{\prime}: S^{1} \times B^{3} \hookrightarrow \mathbb{R} \times \operatorname{int}(Y)$ in a compact part of $\mathbb{R} \times Y$, so that we have $\tilde{X}=X_{f^{ \pm}}$ for $\pm s$ sufficiently large. This perturbation is still gauge equivariant but no longer translation invariant. We use these interpolation data to set up the 4-dimensional version of the perturbed anti-self-duality equation on $\mathbb{R} \times Y$ as described in Section 6 . For critical points $A^{ \pm} \in \mathcal{R}_{f^{ \pm}} \backslash[0]$ from the two Floer chain complexes we consider the space of generalized Floer trajectories

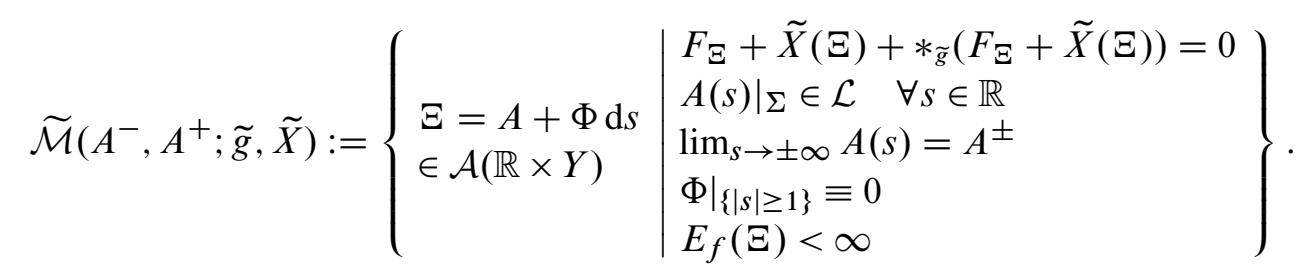

Here $*_{\tilde{g}}$ denotes the Hodge operator on $\mathbb{R} \times Y$ with respect to the metric $\widetilde{g}$. This space is invariant under the gauge group $\mathcal{G}\left(A^{-}, A^{+}\right)$as before, and if the perturbation $\tilde{X}$ is regular, then the quotient $\widetilde{\mathcal{M}}\left(A^{-}, A^{+} ; \tilde{g}, \tilde{X}\right) / \mathcal{G}\left(A^{+}, A^{-}\right)$will be a smooth manifold whose local dimension near $[\mathbb{A}]$ is given by the Fredholm index $\delta(\mathbb{A}) \equiv \mu_{f^{-}}\left(A^{-}\right)-\mu_{f}\left(A^{+}\right)$ (modulo 8). By transversality arguments similar to Section 8 we can find a perturbation $X_{f^{\prime}}$ (and thus $\tilde{X}$ ) such that the linearized operators of index less than or equal to 7 are indeed surjective. Thus we obtain smooth $k$-dimensional moduli spaces

$$
\mathcal{M}^{k}\left(A^{-}, A^{+} ; \tilde{g}, \tilde{X}\right):=\left\{[\mathbb{A}] \in \widetilde{\mathcal{M}}\left(A^{-}, A^{+} ; \tilde{g}, \tilde{X}\right) / \mathcal{G}\left(A^{-}, A^{+}\right) \mid \delta(\mathbb{A})=k\right\}
$$

for $k \leq 7$. The 0 -dimensional moduli spaces are compact by the same analysis as in Section 7. Namely, the main component will converge to a new solution without time-shift; energy cannot be lost by bubbling or by shift to $\pm \infty$ since the remaining solution would have negative index. So - again using the orientations from Section 10 
- we can define a homomorphism

$$
\Phi: \mathrm{CF}\left(Y, \mathcal{L} ; g^{-}, f^{-}\right) \rightarrow \mathrm{CF}\left(Y, \mathcal{L} ; g^{+}, f^{+}\right),
$$

which preserves the grading and is given by

$$
\Phi\left\langle A^{-}\right\rangle:=\sum_{\left.\left[A^{+}\right] \in \mathcal{R}_{f} \backslash \backslash 0\right]}\left(\sum_{\mathbb{A} \in \mathcal{M}^{0}\left(A^{-}, A^{+} ; \tilde{g}, \tilde{X}\right)} v(\mathbb{A})\right)\left\langle A^{+}\right\rangle .
$$

This time the linearized operator is bijective, so $\operatorname{det}\left(\mathcal{D}_{\mathbb{A}}\right)$ is canonically isomorphic to $\mathbb{R}$, and the sign $v(\mathbb{A})= \pm 1$ is obtained by comparing the coherent orientation $o_{\mathbb{A}}$ with the standard orientation of $\mathbb{R}$.

As in the standard theory there are three identities to verify (eg [29, Section 3.2]). First, we must prove that $\Phi$ is a chain map, ie

$$
\partial^{+} \circ \Phi=\Phi \circ \partial^{-} \text {. }
$$

This is proved just like the formula $\partial \circ \partial=0$ in Theorem 11.1. In this case the relevant 1manifold is the moduli space $\mathcal{M}^{1}\left(A^{-}, A^{+} ; \tilde{g}, \tilde{X}\right)$. A compactness and gluing theory similar to Corollary 9.3 identifies the ends of this moduli space with the pairs of trajectories in $\mathcal{M}^{1}\left(A^{-}, B^{-} ; g^{-}, X_{f^{-}}\right) \times \mathcal{M}^{0}\left(B^{-}, A^{+} ; \tilde{g}, \tilde{X}\right)$ for $\left[B^{-}\right] \in \mathcal{R}_{f^{-}} \backslash[0]$ and in $\mathcal{M}^{0}\left(A^{-}, B^{+} ; \tilde{g}, \tilde{X}\right) \times \mathcal{M}^{1}\left(B^{+}, A^{+} ; g^{+}, X_{f}\right)$ for $\left[B^{+}\right] \in \mathcal{R}_{f} \backslash[0]$. Summing over these oriented ends of a 1 -manifold then proves that $\Phi$ satisfies (118) and hence descends to a morphism on Floer homology.

Second, we must prove that the induced map on homology is independent of the choices. Given two such maps $\Phi_{0}, \Phi_{1}: \mathrm{CF}\left(Y, \mathcal{L} ; g^{-}, f^{-}\right) \rightarrow \mathrm{CF}\left(Y, \mathcal{L} ; g^{+}, f^{+}\right)$associated to pairs $\left(\widetilde{g}_{0}, \tilde{X}_{0}\right)$ and $\left(\widetilde{g}_{1}, \tilde{X}_{1}\right)$ we must find a chain homotopy equivalence $H: \mathrm{CF}\left(Y, \mathcal{L} ; g^{-}, f^{-}\right) \rightarrow \mathrm{CF}\left(Y, \mathcal{L} ; g^{+}, f^{+}\right)$satisfying

$$
\Phi_{1}-\Phi_{0}=\partial^{+} \circ H+H \circ \partial^{-} .
$$

To construct $H$ we choose a 1 -parameter family $\left\{\widetilde{g}_{\lambda}, \widetilde{X}_{\lambda}\right\}_{0 \leq \lambda \leq 1}$ of interpolating pairs of metric and perturbation. By Lemma 6.5 the metrics can be interpolated within the space of metrics that are equal to $g_{ \pm}$over the ends and are compatible with the space-time splitting of the boundary. The perturbations $\tilde{X}_{\lambda}$ can be chosen as convex combinations. We then add further compactly supported holonomy perturbations for $0<\lambda<1$ to achieve transversality of the parametrized moduli spaces

$$
\mathcal{M}^{k}\left(A^{-}, A^{+} ;\left\{\tilde{g}_{\lambda}, \tilde{X}_{\lambda}\right\}\right):=\left\{(\lambda,[\mathbb{A}]) \mid[\mathbb{A}] \in \mathcal{M}^{k}\left(A^{-}, A^{+} ; \tilde{g}_{\lambda}, \tilde{X}_{\lambda}\right)\right\}
$$


For $k=-1$ these are compact oriented 0 -manifolds which we use to define $H$ :

$$
H\left\langle A^{-}\right\rangle:=\sum_{\left[A^{+}\right] \in \mathcal{R}_{f_{+} \backslash[0]}}\left(\sum_{(\lambda, \mathbb{A}) \in \mathcal{M}^{-1}\left(A^{-}, A^{+} ;\left\{\tilde{g}_{\lambda}, \tilde{X}_{\lambda}\right\}\right)} v(\lambda, \mathbb{A})\right)\left\langle A^{+}\right\rangle .
$$

The linearized operator has a 1-dimensional cokernel which projects isomorphically to $\mathbb{R}$ and $v(\lambda, \mathbb{A})$ is the sign of this projection. To prove (119) one studies the 1-dimensional moduli space $\mathcal{M}^{0}\left(A^{-}, A^{+} ;\left\{\widetilde{g}_{\lambda}, \tilde{X}_{\lambda}\right\}\right)$ in the usual fashion with the contributions of $\Phi_{0}$ corresponding to the boundary at $\lambda=0$, the contributions of $\Phi_{1}$ to the boundary at $\lambda=1$, and the contributions on the right in (119) to the noncompact ends with $0<\lambda<1$. These ends have either the form of a pair in $\mathcal{M}^{-1}\left(A^{-}, B^{+} ;\left\{\tilde{g}_{\lambda}, \tilde{X}_{\lambda}\right\}\right) \times \mathcal{M}^{1}\left(B^{+}, A^{+} ; g^{+}, X_{f+}\right) / \mathbb{R}$ with $\left[B^{+}\right] \in \mathcal{R}_{f} \backslash\{[0]\}$ or in $\mathcal{M}^{1}\left(A^{-}, B^{-} ; g^{-}, X_{f^{-}}\right) / \mathbb{R} \times \mathcal{M}^{-1}\left(B^{-}, A^{+} ;\left\{\tilde{g}_{\lambda}, \tilde{X}_{\lambda}\right\}\right)$ with $\left[B^{-}\right] \in \mathcal{R}_{f^{-}} \backslash\{[0]\}$. Counting all the ends and boundary points with appropriate signs proves that $H$ satisfies (119).

Third, we must establish the composition rule in (116) for three sets of regular data $\left(g^{\alpha}, f^{\alpha}\right),\left(g^{\beta}, f^{\beta}\right),\left(g^{\gamma}, f^{\gamma}\right)$. We choose regular interpolating metrics and perturbations to define $\Phi^{\beta \alpha}$ and $\Phi^{\gamma \beta}$ on the chain level. The catenation (with gluing parameter $T$ ) of these data gives rise to a regular interpolation from $\left(g^{\alpha}, f^{\alpha}\right)$ to $\left(g^{\gamma}, f^{\gamma}\right)$ for $T$ sufficiently large. The resulting morphism $\Phi_{T}^{\gamma \beta}$ will then, for large $T$, agree with $\Phi^{\gamma \beta} \circ \Phi^{\beta \alpha}$ on the chain level. This follows from a gluing theorem as in Section 9 and compactness arguments as in Theorem 7.7 and Corollary 9.3. In particular, the breaking of connecting trajectories in the limit $T \rightarrow \infty$ at the zero connection is excluded since the stabilizer $\mathcal{G}_{0} \subset \mathcal{G}(Y)$ adds 3 to the index of the glued connection (compare with Remark 9.2 or use index inequalities as in Corollary 7.4.). Again, the orientations are compatible with the gluing by the (Catenation) axiom. The upshot is that, for suitable choices of interpolating data, Equation (116) already holds on the chain level.

Once these three relations have been established one just needs to observe that $\Phi^{\alpha \alpha}$ is the identity on the chain level for the obvious product metric and perturbation on $\mathbb{R} \times Y$. It follows that each $\Phi$ induces an isomorphism on Floer homology. This proves Theorem 11.2.

\section{Appendix A The spectral flow}

In this appendix we adapt the results of Robbin and the first author [26] to families of self-adjoint operators with varying domains. Similar results have appeared in various forms (see Booss-Bavnbeck and Zhu [8], Kirk and Lesch [18] and Ballman, Brüning and Carron [6]). 
Let $H$ be a separable real Hilbert space. Throughout we identify $H$ with its dual space. We consider a family of bounded linear operators

$$
A(s): W(s) \rightarrow H
$$

indexed by $s \in \mathbb{R}$. Here $W(s)$ is a Hilbert space equipped with a compact inclusion $W(s) \subset H$ with a dense image. We formulate conditions under which the unbounded operator

$$
\mathcal{D}:=\partial_{s}+A
$$

on $L^{2}(\mathbb{R}, H)$ is Fredholm and its index is the spectral flow of the operator family $s \mapsto A(s)$. In contrast to [26] the domain of $A(s)$ varies with $s \in \mathbb{R}$. Our axioms give rise to an isomorphic family of operators with constant domain but which are self-adjoint with respect to inner products which vary with $s \in \mathbb{R}$. More precisely, we assume that the disjoint union $\bigsqcup_{s \in \mathbb{R}} W(s)$ is a Hilbert space subbundle of $\mathbb{R} \times H$ in the following sense.

(W1) There is a dense subspace $W_{0} \subset H$ with a compact inclusion and a family of isomorphisms $Q(s): H \rightarrow H$ such that $Q(s) W_{0}=W(s)$ for every $s \in \mathbb{R}$.

(W2) The map $Q: \mathbb{R} \rightarrow \mathcal{L}(H)$ is continuously differentiable in the weak operator topology and there is a $c_{0}>0$ such that, for all $s \in \mathbb{R}$ and $\xi \in W_{0}$,

$$
\begin{aligned}
& c_{0}^{-1}\|\xi\|_{W_{0}} \leq\|Q(s) \xi\|_{W(s)} \leq c_{0}\|\xi\|_{W_{0}}, \\
& \|Q(s) \xi\|_{H}+\left\|\partial_{s} Q(s) \xi\right\|_{H} \leq c_{0}\|\xi\|_{H} .
\end{aligned}
$$

(W3) There exist Hilbert space isomorphisms $Q^{ \pm} \in \mathcal{L}(H)$ such that

$$
\lim _{s \rightarrow \pm \infty}\left\|Q(s)-Q^{ \pm}\right\|_{\mathcal{L}(H)}=0 .
$$

Two trivializations $Q_{1}, Q_{2}: \mathbb{R} \rightarrow \mathcal{L}(H)$ satisfying (W1)-(W3) with $W_{01}, W_{02}$, respectively, are called equivalent if there is a family of Hilbert space isomorphisms $\Phi(s) \in \mathcal{L}(H)$ such that

$$
\Phi(s) W_{01}=W_{02}, \quad Q_{2}(s) \Phi(s)=Q_{1}(s)
$$

for every $s$, the map $\Phi: \mathbb{R} \rightarrow \mathcal{L}(H)$ is continuously differentiable in the weak operator topology, the map $\Phi: \mathbb{R} \rightarrow \mathcal{L}\left(W_{01}, W_{02}\right)$ is continuous in the norm topology, $\sup _{s \in \mathbb{R}}\left\|\partial_{s} \Phi(s)\right\|_{\mathcal{L}(H)}<\infty$, and there exist Hilbert space isomorphisms $\Phi^{ \pm} \in$ $\mathcal{L}(H) \cap \mathcal{L}\left(W_{01}, W_{02}\right)$ such that

$$
\lim _{s \rightarrow \pm \infty}\left\|\Phi(s)-\Phi^{ \pm}\right\|_{\mathcal{L}(H)}=0 .
$$


Remark A.1 To verify (W1)-(W3) it suffices to construct local trivializations on a finite cover $\mathbb{R}=\bigcup U_{\alpha}$ that satisfy these conditions (where condition (W3) is only required near the ends) and that are equivalent over the intersections $U_{\alpha} \cap U_{\beta}$.

We now impose the following conditions on the operator family $A$. Again, it suffices to verify these in the local trivializations of Remark A.1.

(A1) The operators $A(s)$ are uniformly self-adjoint. This means that for each $s \in$ $\mathbb{R}$ the operator $A(s)$ when considered as an unbounded operator on $H$ with $\operatorname{dom} A(s)=W(s)$ is self-adjoint and that there is a constant $c_{1}$ such that

$$
\|\xi\|_{W(s)}^{2} \leq c_{1}\left(\|A(s) \xi\|_{H}^{2}+\|\xi\|_{H}^{2}\right)
$$

for every $s \in \mathbb{R}$ and every $\xi \in W(s)$.

(A2) The map $B:=Q^{-1} A Q: \mathbb{R} \rightarrow \mathcal{L}\left(W_{0}, H\right)$ is continuously differentiable in the weak operator topology and there exists a constant $c_{2}>0$ such that

$$
\|B(s) \xi\|_{H}+\left\|\partial_{s} B(s) \xi\right\|_{H} \leq c_{2}\|\xi\|_{W_{0}}
$$

for every $s \in \mathbb{R}$ and every $\xi \in W_{0}$.

(A3) There are invertible operators $B^{ \pm} \in \mathcal{L}\left(W_{0}, H\right)$ such that

$$
\lim _{s \rightarrow \pm \infty}\left\|B(s)-B^{ \pm}\right\|_{\mathcal{L}\left(W_{0}, H\right)}=0 .
$$

Given a differentiable curve $\xi: \mathbb{R} \rightarrow H$ with $\xi(s) \in W(s)$ for all $s \in \mathbb{R}$ we define $\mathcal{D} \xi: \mathbb{R} \rightarrow H$ by

$$
(\mathcal{D} \xi)(s)=\partial_{s} \xi(s)+A(s) \xi(s) .
$$

This map extends to a bounded linear operator

$$
\mathcal{D}: W^{1,2}(\mathbb{R}, H) \cap L^{2}(\mathbb{R}, W) \rightarrow L^{2}(\mathbb{R}, H) .
$$

Here $L^{2}(\mathbb{R}, W):=\left\{Q \eta_{0} \mid \eta_{0} \in L^{2}\left(\mathbb{R}, W_{0}\right)\right\}$ is a Hilbert space with the norm

$$
\|\eta\|_{L^{2}(\mathbb{R}, W)}^{2}=\int_{-\infty}^{\infty}\|\eta(s)\|_{W(s)}^{2} \mathrm{~d} s .
$$

By (W2) this norm is equivalent to the norm on $L^{2}\left(\mathbb{R}, W_{0}\right)$ under the isomorphism $\eta \mapsto Q^{-1} \eta$. We will prove the following estimate, regularity, and index identity.

Lemma A.2 There exist constants $c$ and $T$ such that

$$
\int_{-\infty}^{\infty}\left(\left\|\partial_{s} \xi(s)\right\|_{H}^{2}+\|\xi(s)\|_{W(s)}^{2}\right) \mathrm{d} s \leq c^{2}\left(\int_{-\infty}^{\infty}\|\mathcal{D} \xi(s)\|_{H}^{2} \mathrm{~d} s+\int_{-T}^{T}\|\xi(s)\|_{H}^{2} \mathrm{~d} s\right)
$$

for every $\xi \in W^{1,2}(\mathbb{R}, H) \cap L^{2}(\mathbb{R}, W)$. 
Theorem A.3 Suppose that $\xi, \eta \in L^{2}(\mathbb{R}, H)$ satisfy

$$
\int_{-\infty}^{\infty}\left(\left\langle\partial_{s} \varphi(s)-A(s) \varphi(s), \xi(s)\right\rangle+\langle\varphi(s), \eta(s)\rangle\right) \mathrm{d} s=0
$$

for every test function $\varphi: \mathbb{R} \rightarrow H$ such that $Q^{-1} \varphi \in \mathcal{C}_{0}^{1}\left(\mathbb{R}, W_{0}\right)$. Then

$$
\xi \in W^{1,2}(\mathbb{R}, H) \cap L^{2}(\mathbb{R}, W), \quad \mathcal{D} \xi=\eta .
$$

Theorem A.4 The operator $\mathcal{D}$ is Fredholm and its index is equal to the upward spectral flow of the operator family $s \mapsto A(s)$.

As in the case of constant domain the spectral flow can be defined as the sum of the crossing indices

$$
\mu_{\text {spec }}(A):=\sum_{s} \operatorname{sign} \Gamma(A, s)
$$

In the present case the crossing form $\Gamma(A, s): \operatorname{ker} A(s) \rightarrow \mathbb{R}$ is defined by

$$
\Gamma(A, s)(\xi):=\left.\frac{d}{d t}\right|_{t=0}\langle\xi(t), A(s+t) \xi(t)\rangle,
$$

where $\xi(t) \in W(s+t)$ is chosen such $\xi(0)=\xi$ and the path $t \mapsto A(s+t) \xi(t) \in H$ is differentiable (for example $\xi(t):=Q(s+t) Q(s)^{-1} \xi$ ); the value of the crossing form at $\xi$ is independent of the choice of the path $t \mapsto \xi(t)$. We assume that the crossings are all regular, ie $\Gamma(A, s)$ is nondegenerate for every $s \in \mathbb{R}$ with $\operatorname{ker} A(s) \neq\{0\}$. Under this assumption the sum in (120) is finite.

Two operator families $A_{1}(s): W_{1}(s) \rightarrow H$ and $A_{2}(s): W_{2}(s) \rightarrow H$ with the same endpoints $A^{ \pm}$are called homotopic if they can be connected by an operator family $A_{\lambda}(s): W_{\lambda}(s) \rightarrow H, 1 \leq \lambda \leq 2$, with the following properties. There is a family of Hilbert space isomorphisms $Q_{\lambda}(s): H \rightarrow H$ that is continuously differentiable in $\lambda$ and $s$ with respect to the weak operator topology and satisfies $Q_{\lambda}(s) W_{0}=W_{\lambda}(s)$ as well as conditions (W2)-(W3) uniformly in $\lambda$. Moreover $A_{\lambda}(s)$ satisfies (A1)(A3) with constants independent of $\lambda$ and the map [1,2] $\times \mathbb{R} \rightarrow \mathcal{L}\left(W_{0}, H\right):(\lambda, s) \mapsto$ $Q_{\lambda}(s)^{-1} A_{\lambda}(s) Q_{\lambda}(s)$ is continuously differentiable in the weak operator topology.

The spectral flow has the following properties:

(Homotopy) The spectral flow is invariant under homotopy.

(Constant) If $W(s)$ and $A(s)$ are independent of $s \in \mathbb{R}$ then $\mu_{\text {spec }}(A)=0$. 
(Direct sum) The spectral flow of a direct sum of two operator families $A$ and $B$ is the sum of their spectral flows, ie

$$
\mu_{\text {spec }}(A \oplus B)=\mu_{\text {spec }}(A)+\mu_{\text {spec }}(B) .
$$

(Catenation) The spectral flow of the catenation of two operator families $A_{01}$ from $A_{0}$ to $A_{1}$ and $A_{12}$ from $A_{1}$ to $A_{2}$ is the sum of their spectral flows, ie

$$
\mu_{\text {spec }}\left(A_{01} \# A_{12}\right)=\mu_{\text {spec }}\left(A_{01}\right)_{+} \mu_{\text {spec }}\left(A_{12}\right) .
$$

(Normalization) For $W=H=\mathbb{R}, A(s)=\arctan (s)$ we have $\mu_{\text {spec }}(A)=1$.

The spectral flow is uniquely determined by the homotopy, constant, direct sum, and normalization axioms. The proof is the same as that of [26, Theorem 4.23] and will be omitted.

Proof of Lemma A.2 The proof is analogous to that of [26, Lemma 3.9]. The only difference is in the first step where we prove the estimate with $T=\infty$. For every $\xi: \mathbb{R} \rightarrow H$ such that $\eta:=Q^{-1} \xi \in \mathcal{C}_{0}^{1}\left(\mathbb{R}, W_{0}\right)$ we have

$$
\int_{-\infty}^{\infty}\|\mathcal{D} \xi\|_{H}^{2} \mathrm{~d} s=\int_{-\infty}^{\infty}\left(\left\|\partial_{s} \xi\right\|_{H}^{2}+\|A \xi\|_{H}^{2}+2\left\langle\partial_{s} \xi, A \xi\right\rangle\right) \mathrm{d} s .
$$

The last summand can be estimated by

$$
\begin{aligned}
2 \int_{-\infty}^{\infty}\left\langle\partial_{s} \xi,\right. & A \xi\rangle \mathrm{d} s \\
& =\int_{-\infty}^{\infty}\left(2\left\langle\left(\partial_{s} Q\right) \eta, A Q \eta\right\rangle+\left\langle Q \partial_{s} \eta, A Q \eta\right\rangle+\left\langle Q \eta, A Q \partial_{s} \eta\right\rangle\right) \mathrm{d} s \\
& =\int_{-\infty}^{\infty}\left(\left\langle\left(\partial_{s} Q\right) \eta, Q B \eta\right\rangle-\left\langle Q \eta,\left(\partial_{s} Q\right) B \eta\right\rangle-\left\langle Q \eta, Q\left(\partial_{s} B\right) \eta\right\rangle\right) \mathrm{d} s \\
& \leq 3 c_{0}^{2} c_{2} \int_{-\infty}^{\infty}\|\eta\|_{H}\|\eta\|_{W_{0}} \mathrm{~d} s \\
& \leq c\|\xi\|_{L^{2}(\mathbb{R}, H)}\|\xi\|_{L^{2}(\mathbb{R}, W)}
\end{aligned}
$$

with $c:=3 c_{0}^{4} c_{2}$. Here we used partial integration and the identity $A Q=Q B$. Now use (A1) to obtain

$$
\begin{aligned}
& \|\mathcal{D} \xi\|_{L^{2}(\mathbb{R}, H)}^{2} \\
& \quad \geq\left\|\partial_{s} \xi\right\|_{L^{2}(\mathbb{R}, H)}^{2}+c_{1}^{-1}\|\xi\|_{L^{2}(\mathbb{R}, W)}^{2}-\|\xi\|_{L^{2}(\mathbb{R}, H)}^{2}-c\|\xi\|_{L^{2}(\mathbb{R}, H)}\|\xi\|_{L^{2}(\mathbb{R}, W)} \\
& \quad \geq\left\|\partial_{s} \xi\right\|_{L^{2}(\mathbb{R}, H)}^{2}+\left(2 c_{1}\right)^{-1}\|\xi\|_{L^{2}(\mathbb{R}, W)}^{2}-\left(1+\frac{1}{2} c^{2} c_{1}\right)\|\xi\|_{L^{2}(\mathbb{R}, H)}^{2}
\end{aligned}
$$

This proves the estimate for $T=\infty$. 
Proof of Theorem A.3 We follow the line of argument in [26, Theorem 3.10].

Step 1 Define $\xi_{0}, \eta_{0} \in L^{2}(\mathbb{R}, H)$ by

$$
\xi_{0}(s):=Q(s)^{*} \xi(s), \quad \eta_{0}(s):=Q(s)^{*} \eta(s)+\left(\partial_{s} Q(s)^{*}\right) \xi(s) .
$$

Then $\xi_{0} \in W^{1,2}\left(\mathbb{R}, W_{0}^{*}\right)$ and

$$
\partial_{s} \xi_{0}(s)=-B(s)^{*} \xi_{0}(s)+\eta_{0}(s) .
$$

To see this we calculate for $\varphi_{0} \in \mathcal{C}_{0}^{\infty}\left(\mathbb{R}, W_{0}\right)$

$$
\begin{aligned}
\int_{-\infty}^{\infty}\left\langle\partial_{s} \varphi_{0}, \xi_{0}\right\rangle_{H} \mathrm{~d} s & =\int_{-\infty}^{\infty}\left\langle\partial_{s}\left(Q \varphi_{0}\right)-\left(\partial_{s} Q\right) \varphi_{0}, \xi\right\rangle_{H} \mathrm{~d} s \\
& =\int_{-\infty}^{\infty}\left(\left\langle A Q \varphi_{0}, \xi\right\rangle_{H}-\left\langle Q \varphi_{0}, \eta\right\rangle_{H}-\left\langle\left(\partial_{s} Q\right) \varphi_{0}, \xi\right\rangle_{H}\right) \mathrm{d} s \\
& =\int_{-\infty}^{\infty}\left(\left\langle\varphi_{0}, B^{*} \xi_{0}-\eta_{0}\right\rangle_{W_{0}, W_{0}^{*}}\right) \mathrm{d} s .
\end{aligned}
$$

Here the self-adjoint operator $A(s)$ extends to an operator in $\mathcal{L}\left(H, W(s)^{*}\right)$ which we also denote by $A(s)$. We denote the dual of the trivialization $Q(s)$ by $Q(s)^{*} \in \mathcal{L}(H)$, which extends to an isomorphism $W(s)^{*} \rightarrow W_{0}^{*}$. With this we can write $B^{*}=$ $Q^{*} A\left(Q^{*}\right)^{-1}$ for the dual operator family of $B=Q^{-1} A Q$, which is continuously differentiable in $\mathcal{L}\left(H, W_{0}^{*}\right)$ with a uniform estimate dual to that in (A2). So we have $B^{*} \xi_{0}-\eta_{0} \in L^{2}\left(\mathbb{R}, W_{0}^{*}\right)$, and since the derivatives of test functions $\varphi_{0}$ are dense in $L^{2}\left(\mathbb{R}, W_{0}\right)$ this implies Step 1 .

Step 2 Suppose that $\xi$ and $\eta$ are supported in an interval $I$ such that for all $s \in I$ the operator $B(s): W_{0} \rightarrow H$ is bijective and satisfies a uniform estimate

$$
\left\|B(s)^{-1}\right\|_{\mathcal{L}\left(H, W_{0}\right)} \leq c .
$$

Fix a smooth function $\rho: \mathbb{R} \rightarrow[0, \infty)$ with support in $(-1,1)$ and $\int \rho=1$ and denote by $\rho_{\delta}(s)=\delta^{-1} \rho\left(\delta^{-1} s\right)$ for $\delta>0$ the standard mollifier. Then we find a constant $C$ such that $\rho_{\delta} *\left(Q^{-1} \xi\right) \in W^{1,2}(\mathbb{R}, H) \cap L^{2}\left(\mathbb{R}, W_{0}\right)$ for all $\delta>0$ and

$$
\left\|\mathcal{D} Q\left(\rho_{\delta} *\left(Q^{-1} \xi\right)\right)\right\|_{L^{2}(\mathbb{R}, H)} \leq C .
$$

Multiply Equation (121) by $\left(B^{*}\right)^{-1}$ to obtain $\xi_{0}=\left(B^{*}\right)^{-1}\left(\eta_{0}-\partial_{s} \xi_{0}\right)$ and note that $Q^{-1} \xi=\left(Q^{*} Q\right)^{-1} \xi_{0}$. Then convolution gives

$$
\begin{aligned}
\rho_{\delta} *\left(Q^{-1} \xi\right) & =\rho_{\delta} *\left(\partial_{s}\left(\left(B^{*} Q^{*} Q\right)^{-1}\right) \xi_{0}+\left(B^{*} Q^{*} Q\right)^{-1} \eta_{0}\right)-\dot{\rho}_{\delta} *\left(\left(B^{*} Q^{*} Q\right)^{-1} \xi_{0}\right) \\
& =\rho_{\delta} * \zeta_{0}-\dot{\rho}_{\delta} *\left(\left(Q^{*} Q B\right)^{-1} \xi_{0}\right)
\end{aligned}
$$


with $\zeta_{0}=\partial_{s}\left(\left(Q^{*} Q B\right)^{-1}\right) \xi_{0}+\left(Q^{*} Q B\right)^{-1} \eta_{0} \in L^{2}\left(\mathbb{R}, W_{0}\right)$. This takes values in $W_{0}$ since

$$
\left(B^{*} Q^{*} Q\right)^{-1}=Q^{-1} A^{-1}\left(Q^{*}\right)^{-1}=B^{-1}\left(Q^{*} Q\right)^{-1}
$$

and its derivative are uniformly bounded in $\mathcal{L}\left(H, W_{0}\right)$.

So, after convolution, $Q\left(\rho_{\delta} *\left(Q^{-1} \xi\right)\right)$ lies in the domain of $\mathcal{D}$ and

$$
\begin{aligned}
Q^{-1} \mathcal{D} Q\left(\rho_{\delta} *\right. & \left.\left(Q^{-1} \xi\right)\right) \\
= & \dot{\rho}_{\delta} *\left(Q^{-1} \xi\right)+Q^{-1}\left(\partial_{s} Q\right)\left(\rho_{\delta} *\left(Q^{-1} \xi\right)\right)+B\left(\rho_{\delta} *\left(Q^{-1} \xi\right)\right) \\
= & B\left(B^{-1}\left(\dot{\rho}_{\delta} *\left(Q^{-1} \xi\right)\right)-\dot{\rho}_{\delta} *\left(\left(Q^{*} Q B\right)^{-1} \xi_{0}\right)\right) \\
& +Q^{-1}\left(\partial_{s} Q\right)\left(\rho_{\delta} *\left(Q^{-1} \xi\right)\right)+B\left(\rho_{\delta} * \zeta_{0}\right)
\end{aligned}
$$

The second line is uniformly bounded in $L^{2}(\mathbb{R}, H)$. For the first term we have

$$
\begin{aligned}
\int_{-\infty}^{\infty}\left\|B^{-1}\left(\dot{\rho}_{\delta} *\left(Q^{-1} \xi\right)\right)(s)-\dot{\rho}_{\delta} *\left(\left(Q^{*} Q B\right)^{-1} \xi_{0}\right)(s)\right\|_{W_{0}} \mathrm{~d} s \\
\quad=\int_{-\infty}^{\infty}\left\|\int_{s-\delta}^{s+\delta} \frac{1}{\delta} \dot{\rho}\left(\frac{t-s}{\delta}\right) \frac{B(s)^{-1}-B(t)^{-1}}{\delta}\left(Q(t)^{*} Q(t)\right)^{-1} \xi_{0}(t) \mathrm{d} t\right\|_{W_{0}} \mathrm{~d} s \\
\leq C \int_{-\infty}^{\infty} \int_{-\infty}^{\infty}\left|\frac{1}{\delta} \dot{\rho}\left(\frac{t-s}{\delta}\right)\right|\left\|\xi_{0}(t)\right\|_{H} \mathrm{~d} t \mathrm{~d} s \\
\leq C\|\dot{\rho}\|_{L^{1}(\mathbb{R})} \int_{-\infty}^{\infty}\left\|\xi_{0}(t)\right\|_{H} \mathrm{~d} t
\end{aligned}
$$

Here the constant $C$ contains a uniform bound for $\partial_{S} B^{-1}=-B^{-1}\left(\partial_{S} B\right) B^{-1}$ on $I$. This proves Step 2.

Step $3 \xi \in W^{1,2}(\mathbb{R}, H) \cap L^{2}(\mathbb{R}, W)$ and $\mathcal{D} \xi=\eta$.

Under the assumptions of Step 2 it follows from Lemma A.2 that $\rho_{\delta} *\left(Q^{-1} \xi\right)$ is uniformly bounded in $\mathcal{W}:=L^{2}\left(\mathbb{R}, W_{0}\right) \cap W^{1,2}(\mathbb{R}, H)$ for all $\delta>0$. So there is a sequence $\delta_{v} \rightarrow 0$ such that $\rho_{\delta_{v}} *\left(Q^{-1} \xi\right)$ converges weakly in $\mathcal{W}$. The limit has to coincide with the strong $L^{2}(\mathbb{R}, H)$-limit $Q^{-1} \xi$. Thus we have $\xi \in L^{2}(\mathbb{R}, W) \cap$ $W^{1,2}(\mathbb{R}, H)$. Now it follows from (121) and (122) that

$$
\begin{aligned}
\mathcal{D} \xi & =\left(Q^{*}\right)^{-1} \partial_{s} \xi_{0}-\left(Q^{*}\right)^{-1}\left(\partial_{s} Q^{*}\right)\left(Q^{*}\right)^{-1} \xi_{0}+B\left(Q^{*}\right)^{-1} \xi_{0} \\
& =\eta-\left(Q^{*}\right)^{-1} B^{*} \xi_{0}+B\left(Q^{*}\right)^{-1} \xi_{0}=\eta .
\end{aligned}
$$

This proves the theorem under the assumption that $\xi$ and $\eta$ are supported in an interval on which $B$ is bijective. In general, one can cover the real axis by finitely many open 
intervals on which $\lambda \mathbb{1}+B(s): W_{0} \rightarrow H$ has uniformly bounded inverses for some $\lambda \in \mathbb{R}$. Then one can use a partition of unity argument to deduce the regularity and equation for $\xi$ on each interval.

Sketch of proof of Theorem A.4 By Lemma A.2 the operator $\mathcal{D}$ has a finite dimensional kernel and a closed image. By Theorem A.3 the cokernel of $\mathcal{D}$ is the kernel of the operator $\mathcal{D}^{\prime}$ with $A$ replaced by $-A$. Hence the cokernel of $\mathcal{D}$ is also finite dimensional and thus $\mathcal{D}$ is Fredholm.

To prove the index identity one verifies as in [26, Theorem 4.1] that the Fredholm index satisfies the (Homotopy), (Constant), (Direct sum) and (Normalization) axioms, which characterize the spectral flow. For the homotopy and the direct sum property one can extend the proofs in [26] without difficulty to nonconstant domains; the constant and normalization properties are immediate since they only refer to constant domains.

We conclude this appendix with a version of the index identity for twisted loops of self-adjoint operators.

Theorem A.5 Let $A(s): W(s) \rightarrow H$ be an operator family that satisfies the conditions (W1)-(W2), (A1)-(A2) and

$$
W(s+1)=Q^{-1} W(s), \quad A(s+1)=Q^{-1} A(s) Q
$$

for every $s \in \mathbb{R}$ and a suitable Hilbert space isomorphism $Q: H \rightarrow H$. Then $A$ induces a Fredholm operator $\mathcal{D}=\partial_{s}+A: \mathcal{W} \rightarrow \mathcal{H}$, where

$$
\begin{aligned}
\mathcal{H} & :=\left\{\xi \in L_{\mathrm{loc}}^{2}(\mathbb{R}, H) \mid \xi(s+1)=Q^{-1} \xi(s)\right\}, \\
\mathcal{W} & :=\left\{\xi \in L_{\mathrm{loc}}^{2}(\mathbb{R}, W) \cap W_{\mathrm{loc}}^{1,2}(\mathbb{R}, H) \mid \xi(s+1)=Q^{-1} \xi(s)\right\} .
\end{aligned}
$$

Its Fredholm index is equal to the upward spectral flow of the operator family $A$ on a fundamental domain $\left[s_{0}, s_{0}+1\right]$.

Proof The Fredholm property follows from Lemma A.2 and Theorem A.3. The proof of the index formula can be reduced to Theorem A.4 by using the homotopy invariance of spectral flow and Fredholm index, stretching the fundamental domain, and comparing kernel and cokernel with a corresponding operator over $\mathbb{R}$ via a gluing argument. We omit the details. For a version of the relevant linear gluing theorem see Donaldson [10, Propositions 3.8, (3.2)]. 


\section{Appendix B Symmetric operators and Fredholm pairs}

Associated to a closed densely defined symmetric operator on a Hilbert space is a (possibly infinite dimensional) symplectic vector space, namely the quotient of the domain of the dual operator by the domain of the original operator. This space can be thought of as the space of boundary data and the symplectic form is obtained from integration by parts. We call this space the Gelfand-Robbin quotient, because the first author learned about this notion from Joel Robbin and Israel Gelfand in the early nineties, who arrived at these ideas independently, but never wrote them up. Though a precursor to this discussion can be found in Dunford and Schwarz [13, Chapter XII.4] we are not aware of an earlier reference explicitly making the connection between self-adjoint operators and symplectic geometry. Since then many researchers have contributed to this field. In particular, the notion of a Fredholm pair of Lagrangian subspaces (of what we call the Gelfand-Robbin quotient) plays a central role in the work of Booss-Bavnbek and his coauthors [7; 8]; they introduced the Maslov index for paths of such Fredholm pairs and related it to the spectral flow in a suitable setting (see Remark B.15 below). The results proved in this appendix play a crucial role in reducing our orientation and index theorems to the case of closed 3-manifolds. We couldn't find these results in the relevant papers, although most of the definitions and some of the basic lemmas below are contained in the existing literature.

Let $H$ be a Hilbert space and $D: \operatorname{dom} D \rightarrow H$ be an injective, symmetric, but not necessarily self-adjoint, operator with a dense domain and a closed image. Then the domain of the adjoint operator $D^{*}$ : dom $D^{*} \rightarrow H$ contains the domain of $D$ and the restriction of $D^{*}$ to the domain of $D$ agrees with $D$. The Gelfand-Robbin quotient

$$
V:=\operatorname{dom} D^{*} / \operatorname{dom} D
$$

carries a natural symplectic form

$$
\omega([x],[y]):=\left\langle D^{*} x, y\right\rangle-\left\langle x, D^{*} y\right\rangle .
$$

The Lagrangian subspaces $\Lambda \subset V$ are in one-to-one correspondence to self-adjoint extensions $D_{\Lambda}$ of $D$ with

$$
\operatorname{dom} D_{\Lambda}:=\left\{x \in \operatorname{dom} D^{*} \mid[x] \in \Lambda\right\} .
$$

Moreover, the kernel of $D^{*}$ determines a Lagrangian subspace

$$
\Lambda_{0}:=\left\{[x] \in V \mid x \in \operatorname{dom} D^{*}, D^{*} x=0\right\} .
$$

The operator $D_{\Lambda}$ is bijective if and only if $V=\Lambda_{0} \oplus \Lambda$. (See Lemma B.3 below.) 
The domain of $D^{*}$ is a Hilbert space with the graph inner product

$$
\langle x, y\rangle_{D^{*}}:=\langle x, y\rangle_{H}+\left\langle D^{*} x, D^{*} y\right\rangle_{H} .
$$

The domain of $D$ is a closed subspace because $D$ has a closed graph. Hence both dom $D$ and the quotient space $V=\operatorname{dom} D^{*} / \operatorname{dom} D$ inherit a Hilbert space structure from dom $D^{*}$. One can now check (using the next remark) that $(V, \omega)$ is a symplectic Hilbert space in the sense that the symplectic form is bounded and the linear map $V \rightarrow V^{*}: v \mapsto I_{\omega}(v):=\omega(v, \cdot)$ is an isomorphism. If $\Lambda \subset V$ is a Lagrangian subspace, ie the annihilator $\Lambda^{\perp} \subset V^{*}$ is given by $\Lambda^{\perp}=I_{\omega}(\Lambda)$, then $\Lambda$ is closed and hence inherits a Hilbert space structure from $V$.

Remark B.1 (i) The graph norm on $\operatorname{dom} D$ is equivalent to the norm

$$
\langle x, y\rangle_{D}:=\langle D x, D y\rangle_{H} .
$$

because $D$ is injective and has a closed image.

(ii) For convenience, we sometimes identify the Gelfand-Robbin quotient $V=$ $\operatorname{dom} D^{*} / \operatorname{dom} D$ with the orthogonal complement

$$
V=(\operatorname{dom} D)^{\perp}=\left\{x \in \operatorname{dom} D^{*} \mid D^{*} x \in \operatorname{dom} D^{*}, D^{*} D^{*} x+x=0\right\} .
$$

The orthogonal projection of $\operatorname{dom} D^{*}$ onto $V$ along $\operatorname{dom} D$ is given by

$$
\operatorname{dom} D^{*} \rightarrow V: x \mapsto x-\left(11+D^{*} D\right)^{-1}\left(x+D^{*} D^{*} x\right),
$$

where $11+D^{*} D$ is understood as an operator from $\operatorname{dom} D$ to $(\operatorname{dom} D)^{*}$. The graph inner product on $V$ is compatible with the symplectic form and the associated complex structure is $x \mapsto J x:=D^{*} x$, that is $\omega(x, J y)=\langle x, y\rangle_{D^{*}}$. This shows that $(V, \omega)$ is indeed a symplectic Hilbert space.

(iii) In the formulation of (ii) the subspace $\Lambda_{0}$ and its orthogonal complement are given by

$$
\begin{aligned}
& \Lambda_{0}=\left\{x \in V \mid \exists \xi \in \operatorname{dom} D \text { s.t. } D^{*}(x+\xi)=0\right\}=\left\{x \in V \mid D^{*} x \in \operatorname{im} D\right\} \\
& \Lambda_{0}^{\perp}=D^{*} \Lambda_{0}=V \cap \operatorname{im} D .
\end{aligned}
$$

Definition B.2 A triple $\left(V, \Lambda_{1}, \Lambda_{2}\right)$ consisting of a Hilbert space $V$ and two closed subspaces $\Lambda_{1}, \Lambda_{2} \subset V$ is called Fredholm if $\Lambda_{1} \cap \Lambda_{2}$ is finite dimensional, $\Lambda_{1}+\Lambda_{2}$ is a closed subspace of $V$, and the cosum $V /\left(\Lambda_{1}+\Lambda_{2}\right)$ is finite dimensional (see Robbin, Ruan and Salamon [24]); equivalently the linear operator $S: \Lambda_{1} \times \Lambda_{2} \rightarrow V$ 
given by $S\left(x_{1}, x_{2}\right):=x_{1}+x_{2}$ is Fredholm. The Fredholm index of a Fredholm triple $\left(V, \Lambda_{1}, \Lambda_{2}\right)$ is defined by

$$
\operatorname{index}\left(V, \Lambda_{1}, \Lambda_{2}\right):=\operatorname{dim}\left(\Lambda_{1} \cap \Lambda_{2}\right)-\operatorname{dim}\left(V /\left(\Lambda_{1}+\Lambda_{2}\right)\right)=\operatorname{index}(S) .
$$

Lemma B.3 Let $\Lambda \subset V$ be a Lagrangian subspace. Then $D_{\Lambda}$ : $\operatorname{dom} D_{\Lambda} \rightarrow H$ is a Fredholm operator if and only if $\left(V, \Lambda_{0}, \Lambda\right)$ is a Fredholm triple.

Proof This follows from the definition and the fact that the homomorphisms

$$
\begin{aligned}
& \operatorname{ker} D_{\Lambda} \rightarrow \Lambda_{0} \cap \Lambda: x \mapsto[x], \\
& \frac{V}{\Lambda_{0}+\Lambda} \rightarrow \frac{H}{\operatorname{im} D_{\Lambda}}:[x] \mapsto\left[D^{*} x\right]
\end{aligned}
$$

are bijective. For the second map this uses Lemma B.4 below.

Lemma B.4 Let $D: \operatorname{dom} D \rightarrow H$ be an injective symmetric operator with a closed image and a dense domain. Then

$$
Y:=\left\{\xi \in \operatorname{dom} D \mid D \xi \in \operatorname{dom} D^{*}\right\}
$$

is a Hilbert space with the inner product

$$
\langle\xi, \eta\rangle_{Y}:=\langle\xi, \eta\rangle_{H}+\langle D \xi, D \eta\rangle_{H}+\left\langle D^{*} D \xi, D^{*} D \eta\right\rangle_{H}
$$

and the operator $D^{*} D: Y \rightarrow H$ is an isomorphism.

Moreover, if the inclusion $\operatorname{dom} D \rightarrow H$ is a compact operator then the operator $D\left(D^{*} D\right)^{-1}: H \rightarrow H$ is compact.

Proof We prove that $Y$ is complete. Let $\xi_{i} \in Y$ be a Cauchy sequence. Then $\xi_{i}$, $D \xi_{i}, D^{*} D \xi_{i}$ are Cauchy sequences in $H$. Define $\xi:=\lim \xi_{i}, x:=\lim D \xi_{i}, y:=$ $\lim D^{*} D \xi_{i}$. Since $D$ and $D^{*}$ have closed graphs we have $\xi \in \operatorname{dom} D, x \in \operatorname{dom} D^{*}$, $D \xi=x$, and $D^{*} x=y$. Hence $\xi \in Y$ and $\xi_{i}$ converges to $\xi$ in $Y$.

That $D^{*} D: Y \rightarrow H$ is injective follows since $D$ is injective and $\left\langle D^{*} D \xi, \xi\right\rangle_{H}=$ $\|D \xi\|_{H}^{2}$ for $\xi \in Y$. Now consider the Gelfand triple

$$
Z \subset H \subset Z^{*}
$$

where $Z:=\operatorname{dom} D$ and $\langle\xi, \eta\rangle_{Z}=\langle D \xi, D \eta\rangle_{H}$. We identify $H$ with its dual space and define the inclusion $H \rightarrow Z^{*}$ as the dual operator of the inclusion $Z \rightarrow H$. We can think of $D: Z \rightarrow H$ as a bounded linear operator and of its adjoint as bounded linear operator $D^{*}: H \rightarrow Z^{*}$. Then $\operatorname{dom} D^{*}=\left\{x \in H \mid D^{*} x \in H\right\}$. Since $D: Z \rightarrow H$ is injective and has a closed image the dual operator $D^{*}: H \rightarrow Z^{*}$ is surjective. Now 
let $y \in H$. Then $y \in Z^{*}$ and hence there exists an element $x \in H$ with $D^{*} x=y$. Since $D^{*} x \in H$ we have $x \in \operatorname{dom} D^{*}$. Now it follows from the definitions that the kernel of $D^{*}$ is the orthogonal complement of the image of $D$. Since the image of $D$ is closed this implies $H=\operatorname{ker} D^{*} \oplus \operatorname{im} D$. Hence there is a vector $x_{0} \in \operatorname{ker} D^{*}$ such that $x-x_{0} \in \operatorname{im} D$. Choose $\xi \in \operatorname{dom} D$ such that $D \xi=x-x_{0}$. Then $D \xi \in \operatorname{dom} D^{*}$ and $D^{*} D \xi=D^{*} x=y$. This proves that $D^{*} D$ is surjective.

Now assume that the inclusion $Z \rightarrow H$ is compact. To prove that the operator $D\left(D^{*} D\right)^{-1}: H \rightarrow H$ is compact we observe that

$$
\|x\|_{Z^{*}}:=\sup _{0 \neq \xi \in \operatorname{dom} D} \frac{\langle x, \xi\rangle_{H}}{\|D \xi\|_{H}}=\left\|D\left(D^{*} D\right)^{-1} x\right\|_{H}
$$

for every $x \in H \subset Z^{*}$. Here the last equation follows from the fact that the supremum in the second term is attained at the vector $\xi_{0}=\left(D^{*} D\right)^{-1} x$ with $x=D^{*} D \xi_{0}$. Now let $x_{i}$ be a bounded sequence in $H$. Since the inclusion $H \rightarrow Z^{*}$ is compact, there exists a subsequence $x_{i_{k}}$ which converges in $Z^{*}$ and it follows from (124) that the sequence $D\left(D^{*} D\right)^{-1} x_{i_{k}}$ converges in $H$. This proves the lemma.

Remark B.5 (i) By Lemma B.4 the subspaces $\Lambda_{0}$ and $\Lambda_{0}^{\perp}$ of $V$ in Remark B.1 can also be written in the form

$$
\begin{aligned}
& \Lambda_{0}=\left\{x \in \operatorname{dom} D^{*} \mid D^{*} x+D\left(D^{*} D\right)^{-1} x=0\right\}=\operatorname{ker}\left(D^{*}+T\right), \\
& \Lambda_{0}^{\perp}=\left\{x \in V \mid x=D\left(D^{*} D\right)^{-1} D^{*} x\right\}=T \operatorname{ker}\left(D^{*}+T\right),
\end{aligned}
$$

where $T:=D\left(D^{*} D\right)^{-1}: H \rightarrow H$ maps to $\operatorname{im} T=\operatorname{dom} D^{*} \cap \operatorname{im} D$.

(ii) The orthogonal projection of $V$ onto $\Lambda_{0}^{\perp}$ extends to a bounded linear operator $\Pi_{0}: H \rightarrow H$ given by

$$
\Pi_{0} x=D\left(D^{*} D+T D\right)^{-1}\left(D^{*} x+T x\right) .
$$

Here $D^{*} D+T D: \operatorname{dom} D \rightarrow(\operatorname{dom} D)^{*}$ is an isomorphism because

$$
\left\langle x, D^{*} D x+T D x\right\rangle=\|D x\|^{2}+\|T D x\|^{2} \geq \delta\|x\|^{2} .
$$

In fact, $\Pi_{0}$ is a projection on all of $H$, its kernel is $\Lambda_{0}$, and its image is equal to the image of $D$. In particular, $\left.\Pi_{0}\right|_{\Lambda_{0}^{\perp}}=11$.

(iii) In all our applications the inclusion $\operatorname{dom} D \rightarrow H$ is a compact operator. Then, by Lemma B.4, $T: H \rightarrow H$ is compact, and thus the inclusion $\Lambda_{0}^{\perp} \rightarrow H$ is compact. Indeed, the inclusion is given by the composition $x \mapsto T D^{*} x$ of a compact and a bounded operator. 
The inclusions dom $D^{*} \rightarrow H$ and $\Lambda_{0} \rightarrow H$, however, are not compact unless $V$ is finite dimensional. Namely, if $V$ is infinite dimensional then so is the kernel of $D^{*}$ (since $\Lambda_{0} \subset V$ is Lagrangian) and the inclusion $\operatorname{dom} D^{*} \supset \operatorname{ker} D^{*} \rightarrow H$ is an isometric embedding. Lemma B.10 below gives a condition under which the domain of a self-adjoint extension of $D$ has a compact embedding into $H$. This requires the notion of a compact perturbation of a closed subspace of $V$.

Definition B.6 Let $V$ be a Hilbert space and $\Lambda \subset V$ be a closed subspace. A closed subspace $\Lambda^{\prime} \subset V$ is called a compact perturbation of $\Lambda$ if the projection of $\Lambda^{\prime}$ onto some (and hence every) complement of $\Lambda$ in $V$ is a compact operator and vice versa.

Remark B.7 The notion of compact perturbation defines an equivalence relation on the set of closed subspaces of $V$. To see this denote by $\Pi: V \rightarrow \Lambda$ and $\Pi^{\prime}: V \rightarrow \Lambda^{\prime}$ the orthogonal projections. If $\Lambda^{\prime}$ is a compact perturbation of $\Lambda$ and $\Lambda^{\prime \prime}$ is a compact perturbation of $\Lambda^{\prime}$ then the operators $\mathbb{1 1}-\Pi: \Lambda^{\prime} \rightarrow \Lambda^{\perp}$ and $\mathbb{1 l}-\Pi^{\prime}: \Lambda^{\prime \prime} \rightarrow\left(\Lambda^{\prime}\right)^{\perp}$ are compact. Hence the operator $\left.(\mathbb{1}-\Pi)\right|_{\Lambda^{\prime \prime}}=\left.(\mathbb{1}-\Pi)\left(\mathbb{1}-\Pi^{\prime}\right)\right|_{\Lambda^{\prime \prime}}+\left.(\mathbb{1}-\Pi) \Pi^{\prime}\right|_{\Lambda^{\prime \prime}}$ is compact. Repeating this argument with $\Lambda$ and $\Lambda^{\prime \prime}$ interchanged we see that $\Lambda^{\prime \prime}$ is a compact perturbation of $\Lambda$.

Lemma B.8 Let $V$ be a Hilbert space and $\Lambda_{1}, \Lambda, \Lambda^{\prime} \subset V$ be closed subspaces such that $\Lambda^{\prime}$ is a compact perturbation of $\Lambda$. If $\left(V, \Lambda_{1}, \Lambda\right)$ is a Fredholm triple then so is $\left(V, \Lambda_{1}, \Lambda^{\prime}\right)$.

Proof Let $\Pi: V \rightarrow \Lambda$ and $\Pi^{\prime}: V \rightarrow \Lambda^{\prime}$ be the orthogonal projections. Then (11$\left.\Pi \Pi^{\prime}\right)\left.\right|_{\Lambda}=\left.\Pi\left(\mathbb{1 l}-\Pi^{\prime}\right)\right|_{\Lambda}: \Lambda \rightarrow \Lambda$ and $\left.\left(\mathbb{1 l}-\Pi^{\prime} \Pi\right)\right|_{\Lambda^{\prime}}: \Lambda^{\prime} \rightarrow \Lambda^{\prime}$ are compact operators. This implies that $\left.\Pi\right|_{\Lambda^{\prime}}: \Lambda^{\prime} \rightarrow \Lambda$ and $\left.\Pi^{\prime}\right|_{\Lambda}: \Lambda \rightarrow \Lambda^{\prime}$ are Fredholm operators with opposite indices; see eg [17, Chapter III.3].

Now suppose that $\left(V, \Lambda_{1}, \Lambda\right)$ is a Fredholm triple, ie the map $S: \Lambda_{1} \times \Lambda \rightarrow V$ given by $S\left(v_{1}, v\right)=v_{1}+v$ is Fredholm. Then the operator

$$
S^{\prime \prime}:=S \circ(11 \times \Pi): \Lambda_{1} \times \Lambda^{\prime} \rightarrow V
$$

is Fredholm. Define the map $S^{\prime}: \Lambda_{1} \times \Lambda^{\prime} \rightarrow V$ by $S^{\prime}\left(v_{1}, v^{\prime}\right)=v_{1}+v^{\prime}$. Since $S^{\prime}\left(v_{1}, v^{\prime}\right)-S^{\prime \prime}\left(v_{1}, v^{\prime}\right)=\left(11-\Pi \Pi^{\prime}\right) v^{\prime}$ the operator $S^{\prime}-S^{\prime \prime}$ is compact. Hence $S^{\prime}$ is a Fredholm operator and so $\left(V, \Lambda_{1}, \Lambda^{\prime}\right)$ is a Fredholm triple.

Lemma B.9 Let $(V, \omega)$ be a symplectic Hilbert space. Let $\Lambda, \Lambda^{\prime} \subset V$ be Lagrangian subspaces. Then the following are equivalent.

(i) $\Lambda^{\prime}$ is a compact perturbation of $\Lambda$. 
(ii) The projection of $\Lambda^{\prime}$ onto $\Lambda^{\perp}$ is a compact operator.

(iii) The operator $\Lambda^{\prime} \rightarrow \Lambda^{*}: v^{\prime} \mapsto \omega\left(v^{\prime}, \cdot\right)$ is compact.

Proof By definition, (i) implies (ii). The Lagrangian condition asserts that the orthogonal complement $\Lambda^{\perp}$ is isomorphic to $\Lambda^{*}$ via the isomorphism $\Lambda^{\perp} \rightarrow \Lambda^{*}: v \mapsto \omega(v, \cdot)$. Under this isomorphism the orthogonal projection $\Lambda^{\prime} \rightarrow \Lambda^{\perp}$ corresponds to the operator $\Lambda^{\prime} \rightarrow \Lambda^{*}: v^{\prime} \mapsto \omega\left(v^{\prime}, \cdot\right)$, hence (ii) and (iii) are equivalent. To see that (iii) implies (i) note that the operators $\Lambda^{\prime} \rightarrow \Lambda^{*}: v^{\prime} \mapsto \omega\left(v^{\prime}, \cdot\right)$ and $\Lambda \rightarrow\left(\Lambda^{\prime}\right)^{*}: v \mapsto-\omega(v, \cdot)$ are dual to each other. Using "(iii) $\Leftrightarrow$ (ii)" we see that (iii) implies compactness of both projections $\Lambda^{\prime} \rightarrow \Lambda^{\perp}$ and $\Lambda \rightarrow\left(\Lambda^{\prime}\right)^{\perp}$. This proves the lemma.

Lemma B.10 Let $D: \operatorname{dom} D \rightarrow H$ be an injective symmetric operator with a closed image and a dense domain and suppose that the inclusion $\operatorname{dom} D \rightarrow H$ is a compact operator. Let $V=(\operatorname{dom} D)^{\perp}$ be the Gelfand-Robbin quotient, $\Lambda \subset V$ be a Lagrangian subspace, and $\Lambda_{0}, \Lambda_{0}^{\perp}$ be as in Remark B.1. Then the following are equivalent.

(i) The inclusion dom $D_{\Lambda} \rightarrow H$ is compact.

(ii) The inclusion $\Lambda \rightarrow H$ is compact.

(iii) $\Lambda$ is a compact perturbation of $\Lambda_{0}^{\perp}$.

Proof Let $\Pi_{0}: V \rightarrow V$ denote the orthogonal projection onto $\Lambda_{0}^{\perp}$. Then $\Pi_{0}: V \rightarrow H$ is compact since the inclusion of the image $\Pi_{0}(V)=\Lambda_{0}^{\perp}$ into $H$ is compact by Remark B.5 (ii). By Lemma B.9, (iii) holds if and only if the operator $\left.\left(11-\Pi_{0}\right)\right|_{\Lambda}: \Lambda \rightarrow \Lambda_{0}$ is compact. Moreover, the graph norm of $D^{*}$ on $\Lambda_{0}=\operatorname{ker}\left(D^{*}+T\right)$ is equivalent to the norm of $H$ so, in fact, (iii) holds if and only if the operator $\left.\left(\mathbb{1 l}-\Pi_{0}\right)\right|_{\Lambda}: \Lambda \rightarrow H$ is compact. We deduce that (iii) is equivalent to (ii) because the inclusion $\Lambda \rightarrow H$ is given by the sum $\left.\mathbb{1}\right|_{\Lambda}=\left.\left(\mathbb{1}-\Pi_{0}\right)\right|_{\Lambda}+\left.\Pi_{0}\right|_{\Lambda}$, where $\left.\Pi_{0}\right|_{\Lambda}: \Lambda \rightarrow H$ is compact.

That (i) is equivalent to (ii) follows from the fact that the inclusion of dom $D$ into $H$ is compact, by assumption, and $\operatorname{dom} D_{\Lambda}=\operatorname{dom} D \oplus \Lambda$.

Lemma B.11 Let $D: \operatorname{dom} D \rightarrow H$ be an injective symmetric operator with a closed image and a dense domain and suppose that the inclusion $\operatorname{dom} D \rightarrow H$ is a compact operator. Let $V=\operatorname{dom} D^{*} / \operatorname{dom} D$ be the Gelfand-Robbin quotient and $\Lambda_{0}=\{[x] \in$ $\left.V \mid D^{*} x=0\right\}$ as in (123). Let $P: H \rightarrow H$ be a self-adjoint bounded linear operator such that $D+P: \operatorname{dom} D \rightarrow H$ is injective. Then the following are equivalent.

(i) The composition of $P$ with the inclusion $\operatorname{dom} D^{*} \rightarrow H$ is a compact operator.

(ii) The operator $\left.P\right|_{\operatorname{ker}\left(D^{*}+P\right)}: \operatorname{ker}\left(D^{*}+P\right) \rightarrow H$ is compact. 
(iii) $\Lambda_{P}:=\left\{[x] \in V \mid D^{*} x+P x=0\right\}$ is a compact perturbation of $\Lambda_{0}$.

Proof Abbreviate $D_{P}:=D+P$. Then $\operatorname{dom} D_{P}^{*}=\operatorname{dom} D^{*}$ and the graph norm of $D^{*}$ is equivalent to the graph norm of $D_{P}^{*}$. Moreover, on $\operatorname{ker} D_{P}^{*}$ and $\operatorname{ker} D^{*}$ both graph norms are equivalent to the norm of $H$. For $[x] \in \Lambda_{P}$, represented by $x \in \operatorname{ker} D_{P}^{*}$, and $\left[x_{0}\right] \in \Lambda_{0}$, represented by $x_{0} \in \operatorname{ker} D^{*}$, we have

$$
\omega\left([x],\left[x_{0}\right]\right)=\left\langle D^{*} x, x_{0}\right\rangle-\left\langle x, D^{*} x_{0}\right\rangle=-\left\langle P x, x_{0}\right\rangle=\left\langle T P x-P x, x_{0}\right\rangle,
$$

where $T:=D\left(D^{*} D\right)^{-1}: H \rightarrow \operatorname{dom} D^{*}$. Using Lemma B.9 and the compactness of the inclusion dom $D \rightarrow H$, we see that $\Lambda_{P}$ is a compact perturbation of $\Lambda_{0}$ if and only if $\left.(P-T P)\right|_{\operatorname{ker} D_{P}^{*}}: \operatorname{ker} D_{P}^{*} \rightarrow \operatorname{ker} D^{*}$ is a compact operator. Since $T$ is compact, by Lemma B.4, this shows that (ii) is equivalent to (iii). That (i) implies (ii) is obvious. To prove that (ii) implies (i) note that, by Remark B.5 with $D$ replaced by $D_{P}$, the inclusion of dom $D^{*} \cap \operatorname{im} D_{P}$ into $H$ is compact. Since the decomposition $\operatorname{dom} D^{*}=\left(\operatorname{dom} D^{*} \cap \operatorname{im} D_{P}\right) \oplus \operatorname{ker} D_{P}^{*}$ is orthogonal with respect to the graph norm of $D_{P}^{*}$, this shows that (ii) implies (i).

Remark B.12 Let $D, V, \Lambda_{0}$ be as in Lemma B.11, $P: H \rightarrow H$ be a bounded self-adjoint operator, and denote $\Lambda_{P}:=\left\{[x] \in V \mid D^{*} x+P x=0\right\}$.

(i) Let $\Lambda_{P}^{\perp, P}$ denote the orthogonal complement of $\Lambda_{P}$ with respect to the graph inner product of $D^{*}+P$. Then it always is a compact perturbation of $\Lambda_{0}^{\perp}$. Namely, by Remark B.5 with $D$ replaced by $D+P$, the inclusion $\Lambda_{P}^{\perp, P} \rightarrow H$ is compact. Hence, by Lemma B.10 with $D$ replaced by $D+P$ and $\Lambda:=\Lambda_{P}^{\perp, P}$, the inclusion $\left\{v \in \operatorname{dom} D^{*} \mid[v] \in \Lambda\right\} \rightarrow H$ is compact. Using Lemma B.10 again we deduce that $\Lambda$ is a compact perturbation of $\Lambda_{0}^{\perp}$.

(ii) The orthogonal complement $\Lambda_{P}^{\perp}$ with respect to the graph inner product of $D^{*}$ is a compact perturbation of $\Lambda_{0}^{\perp}$ if and only if the restriction of $P$ to $\operatorname{dom} D^{*}$ is a compact operator. This follows from Lemma B.11 and the fact that $\Lambda_{P}^{\perp}=D^{*} \Lambda_{P}$ and $\Lambda_{0}^{\perp}=D^{*} \Lambda_{0}$ in the notation of Remark B.1, where $D^{*}$ is a compatible complex structure on $V$.

(iii) It follows from (i) and (ii) that $\Lambda_{P}^{\perp, P}$ is a compact perturbation of $\Lambda_{P}^{\perp}$ if and only if the restriction of $P$ to the domain of $D^{*}$ is a compact operator.

(iv) If $\Lambda$ is a compact perturbation of $\Lambda_{0}^{\perp}$ then $\left(V, \Lambda_{P}, \Lambda\right)$ is a Fredholm triple. Since $\left(V, \Lambda_{P}, \Lambda_{P}^{\perp, P}\right)$ is a Fredholm triple, this follows from (i) and Lemma B.8.

Lemma B.13 Let $D, V, \Lambda_{0}$ be as in Lemma $B .11$ and let $P(s): H \rightarrow H$ for $s \in \mathbb{R}$ be a continuously differentiable family of self-adjoint bounded linear operators. Assume 
that $P(s)$ converges to $P( \pm \infty)=: P^{ \pm}$in the operator norm as $s$ tends to $\pm \infty$, that $\left.(D+P(s))\right|_{\operatorname{dom} D}$ is injective for every $s \in \mathbb{R} \cup\{ \pm \infty\}$, and that

$$
\frac{\operatorname{ker}\left(D^{*}+P^{-}\right) \oplus \operatorname{dom} D}{\operatorname{dom} D}=\frac{\operatorname{ker}\left(D^{*}+P^{+}\right) \oplus \operatorname{dom} D}{\operatorname{dom} D}=: \Lambda_{0}^{\prime} \subset V .
$$

Then the spectral flow of the operator family $s \mapsto(D+P(s))_{\Lambda}$ is independent of the Lagrangian subspace $\Lambda \subset V$ such that $V=\Lambda_{0}^{\prime} \oplus \Lambda$ and $\Lambda$ is a compact perturbation of $\Lambda_{0}^{\perp}$.

Remark B.14 Let $D, V, \Lambda_{0}$ be as in Lemma B.11, and let $Q: H \rightarrow H$ be a Hilbert space isomorphism such that

$$
x \in \operatorname{dom} D^{*} \quad \Longrightarrow \quad x-Q x \in \operatorname{dom} D .
$$

Then $Q$ induces the identity on $V$. Let $P(s): H \rightarrow H$ for $s \in \mathbb{R}$ be a continuously differentiable family of self-adjoint bounded linear operators such that

$$
D+P(s+1)=Q^{-1}(D+P(s)) Q .
$$

Assume $\left.(D+P(s))\right|_{\operatorname{dom} D}$ is injective for every $s \in \mathbb{R}$ and denote

$$
\Lambda_{0}^{\prime}:=\frac{\operatorname{ker}\left(D^{*}+P(0)\right) \oplus \operatorname{dom} D}{\operatorname{dom} D}=\frac{\operatorname{ker}\left(D^{*}+P(1)\right) \oplus \operatorname{dom} D}{\operatorname{dom} D} .
$$

Then the spectral flow of the operator family $s \mapsto(D+P(s))_{\Lambda}$ on the fundamental domain $[0,1]$ is independent of the Lagrangian subspace $\Lambda \subset V$ such that $V=\Lambda_{0}^{\prime} \oplus \Lambda$ and $\Lambda$ is a compact perturbation of $\Lambda_{0}^{\perp}$. The proof is the same as that of Lemma B.13.

Proof of Lemma B.13 The operators $D+P(s)$ : $\operatorname{dom} D \rightarrow H$ satisfy the assumptions of this section and give rise to the constant Gelfand-Robbin quotient $V=$ $\operatorname{dom} D^{*} / \operatorname{dom} D$ since $\operatorname{dom}(D+P(s))^{*}=\operatorname{dom} D^{*}$. Hence any Lagrangian subspace $\Lambda \subset V$ gives rise to a family of self-adjoint operators $A(s):=(D+P(s))_{\Lambda}: \operatorname{dom} D_{\Lambda} \rightarrow$ $H$, which satisfies the conditions (A1)-(A3) of Appendix A whenever $V=\Lambda_{0}^{\prime} \oplus \Lambda$. In particular, the estimate in (A1) holds for $s= \pm \infty$, ie $\|x\|_{D^{*}} \leq C\left\|\left(D+P^{ \pm}\right)_{\Lambda} x\right\|_{H}$ for $x \in \operatorname{dom} D_{\Lambda}$, because $\left(V, \Lambda_{0}^{\prime}, \Lambda\right)$ is a Fredholm triple and $\left(D+P^{ \pm}\right)_{\Lambda}$ is injective. The estimate for $s \in \mathbb{R}$ follows from a uniform bound of the form $\left\|P(s)-P^{ \pm}\right\| \leq C$ for the operator norm on $H$. The assumptions (W1)-(W3) are satisfied with the trivial map $Q \equiv \mathbb{1}$ and the constant domain $W_{0}=\operatorname{dom} D_{\Lambda}$. In particular, the domain embeds compactly to $H$, by Lemma B.10, whenever $\Lambda$ is a compact perturbation of $\Lambda_{0}^{\perp}$. Hence the spectral flow is well defined under our assumptions (see Appendix A).

We prove that the set $\mathcal{S}$ of Lagrangian subspaces of $V$ that are transverse to $\Lambda_{0}^{\prime}$ and are compact perturbations of $\Lambda_{0}^{\perp}$ is connected. For that purpose let $\Lambda_{1} \subset V$ denote 
the orthogonal complement of $\Lambda_{0}^{\prime}$ with respect to the graph inner product of $D^{*}+P^{+}$ and let $I_{\omega}: \Lambda_{0}^{\prime} \rightarrow \Lambda_{1}^{*}$ be the isomorphism given by $v \mapsto \omega(v, \cdot)$. Then a subspace $\Lambda \subset V=\Lambda_{1} \oplus \Lambda_{0}^{\prime}$ is a complement of $\Lambda_{0}^{\prime}$ if and only if it is the graph of a linear operator from $\Lambda_{1}$ to $\Lambda_{0}^{\prime}$ or, equivalently, $\Lambda=\Lambda_{A}:=\operatorname{graph}\left(I_{\omega}^{-1} \circ A\right)$ for some linear operator $A: \Lambda_{1} \rightarrow \Lambda_{1}^{*}$. One can check that the subspace $\Lambda_{A}$ is Lagrangian if and only if $A$ is self-adjoint and that it is a compact perturbation of $\Lambda_{0}^{\perp}$ if and only if $A$ is compact. The last assertion uses the explicit formula $x+I_{\omega}^{-1} A x \mapsto I_{\omega}^{-1} A x$ for the projection $\Lambda_{A} \rightarrow \Lambda_{0}^{\prime}$ along $\Lambda_{1}$ and the fact that $\Lambda_{A}$ is a compact perturbation of $\Lambda_{0}^{\perp}$ if and only if it is a compact perturbation of $\Lambda_{1}$, by Remark B.12 (i) and Remark B.7. Thus we have identified $\mathcal{S}$ with the vector space of compact self-adjoint operators $A: \Lambda_{1} \rightarrow \Lambda_{1}^{*}$ and so $\mathcal{S}$ is contractible, as claimed.

Now the result follows from the homotopy invariance of the spectral flow. The homotopies of Lagrangian subspaces do not directly translate into homotopies in the sense of Appendix A; see the proof of Lemma B.16 below. However, the homotopy invariance of the spectral flow of the family $s \mapsto(D+P(s))_{\Lambda}$ follows from Remark B.15, where the spectral flow is identified with a Maslov index, which in turn is invariant under homotopies of $\Lambda$.

Remark B.15 (i) Let $[0,1] \ni s \mapsto\left(\Lambda_{0}(s), \Lambda_{1}(s)\right)$ be a smooth path of pairs of Lagrangian subspaces of $V$ such that $\left(V, \Lambda_{0}(s), \Lambda_{1}(s)\right)$ is a Fredholm triple for every $s$. For each $s$ define the crossing form $\Gamma\left(\Lambda_{0}, \Lambda_{1}, s\right): \Lambda_{0}(s) \cap \Lambda_{1}(s) \rightarrow \mathbb{R}$ by

$$
\Gamma\left(\Lambda_{0}, \Lambda_{1}, s\right)(v):=\left.\frac{\mathrm{d}}{\mathrm{d} t}\right|_{t=0}\left(\omega\left(v, v_{0}^{\prime}(t)\right)-\omega\left(v, v_{1}^{\prime}(t)\right)\right)
$$

for $v \in \Lambda_{0}(s) \cap \Lambda_{1}(s)$, where $\Lambda_{0}^{\prime}, \Lambda_{1}^{\prime} \subset V$ are Lagrangian subspaces such that $V=\Lambda_{0}(s) \oplus \Lambda_{0}^{\prime}=\Lambda_{1}(s) \oplus \Lambda_{1}^{\prime}$ and $v_{0}^{\prime}(t) \in \Lambda_{0}^{\prime}, v_{1}^{\prime}(t) \in \Lambda_{1}^{\prime}$ are chosen such that $v+v_{0}^{\prime}(t) \in \Lambda_{0}(s+t)$ and $v+v_{1}^{\prime}(t) \in \Lambda_{1}(s+t)$. As in [25] the Maslov index is defined as the sum of the signatures of the crossing forms

$$
\mu\left(\Lambda_{0}, \Lambda_{1}\right):=\sum_{s} \operatorname{sign} \Gamma\left(\Lambda_{0}, \Lambda_{1}, s\right)
$$

provided that the crossing forms are all nondegenerate and $\Lambda_{0}(s)$ is transverse to $\Lambda_{1}(s)$ for $s=0,1$. Under this assumption the sum is finite. The nondegeneracy condition can be achieved by a small perturbation with fixed endpoints. The Maslov index is invariant under homotopies of paths of Lagrangian Fredholm triples with transverse endpoints.

(ii) The spectral flow in Lemma B.13 can be identified with the Maslov index

$$
\mu_{\text {spec }}\left((D+P)_{\Lambda}\right)=\mu\left(\Lambda_{P}, \Lambda\right)
$$


where $\Lambda_{0}(s):=\Lambda_{P(s)}=\left\{[x] \in V \mid D^{*} x+P(s) x=0\right\}$ and $\Lambda_{1}(s):=\Lambda$ for every $s$. The Fredholm property of the triples $\left(V, \Lambda_{P(s)}, \Lambda\right)$ follows from Remark B.12 (iv).

To prove (125), fix a real number $s$, choose $\Lambda_{0}^{\prime}$ and $v_{0}^{\prime}(t)$ as in (i), let $x_{0}(t) \in \operatorname{dom} D^{*}$ be the smooth path defined by $\left(D^{*}+P(s+t)\right) x_{0}(t)=0$ and $\left[x_{0}(t)\right]=v+v_{0}^{\prime}(t) \in$ $\Lambda_{0}(s+t)$, and denote $x:=x_{0}(0)$ so that $[x]=v \in \Lambda_{0}(s) \cap \Lambda$. Then

$$
\begin{aligned}
\left.\frac{\mathrm{d}}{\mathrm{d} t}\right|_{t=0} \omega\left(v, v_{0}^{\prime}(t)\right) & =\left.\frac{\mathrm{d}}{\mathrm{d} t}\right|_{t=0} \omega\left(v, v+v_{0}^{\prime}(t)\right) \\
& =\left.\frac{\mathrm{d}}{\mathrm{d} t}\right|_{t=0}\left(\left\langle D^{*} x, x_{0}(t)\right\rangle-\left\langle x, D^{*} x_{0}(t)\right\rangle\right) \\
& =\left.\frac{\mathrm{d}}{\mathrm{d} t}\right|_{t=0}\left\langle\left(D^{*}+P(s+t)\right) x, x_{0}(t)\right\rangle \\
& =\left.\frac{\mathrm{d}}{\mathrm{d} t}\right|_{t=0}\left\langle\left(D^{*}+P(s+t)\right) x, x\right\rangle .
\end{aligned}
$$

This shows that the crossing forms $\Gamma\left(\left(D^{*}+P\right)_{\Lambda}, s\right)$ and $-\Gamma\left(\Lambda_{0}, \Lambda, s\right)$ agree under the isomorphism $\operatorname{ker}\left(D^{*}+P(s)\right)_{\Lambda} \rightarrow \Lambda_{0}(s) \cap \Lambda: x \mapsto[x]$.

Lemma B.16 Let $D, V, \Lambda_{0}, Q, P$ be as in Remark B.14. Denote by $\Upsilon$ the set of Lagrangian subspaces $\Lambda \subset V$ that are compact perturbations of $\Lambda_{0}^{\perp}$. For every $\Lambda \in \Upsilon$ there is a Fredholm operator

$$
\mathcal{D}_{\Lambda}:=\partial_{s}+D_{\Lambda}+P(s): \mathcal{W}_{\Lambda} \rightarrow \mathcal{H}
$$

with $\mathcal{H}:=\left\{\xi \in L_{\text {loc }}^{2}(\mathbb{R}, H) \mid \xi(s+1)=Q^{-1} \xi(s)\right\}$,

$$
\mathcal{W}_{\Lambda}:=\left\{\xi \in L_{\text {loc }}^{2}\left(\mathbb{R}, \operatorname{dom} D_{\Lambda}\right) \cap W_{\text {loc }}^{1,2}(\mathbb{R}, H) \mid \xi(s+1)=Q^{-1} \xi(s)\right\} .
$$

The determinants $\operatorname{det}\left(\mathcal{D}_{\Lambda}\right)$ for $\Lambda \in \Upsilon$ form a line bundle over $\Upsilon$.

Proof $\mathcal{D}_{\Lambda}$ is Fredholm since it is the operator of Theorem A.5 with $A(s)=D_{\Lambda}+P(s)$ and constant domain $W(s)=\operatorname{dom} D_{\Lambda}$.

We do not know if for any two subspaces $\Lambda, \Lambda^{\prime} \in \Upsilon$ there is a Hilbert space isomorphism of $Q: H \rightarrow H$ that identifies dom $D_{\Lambda}$ with dom $D_{\Lambda^{\prime}}$, as would be required for a homotopy of operator families in the sense of Appendix A. However, one can prove directly that the kernel of $\mathcal{D}_{\Lambda}$ depends continuously on $\Lambda$ (as a subspace of $\mathcal{H}$ ) if $\mathcal{D}_{\Lambda}$ is surjective. This proves the lemma since the transverse situation can always be achieved by finite dimensional stabilization.

To prove the continuous dependence of $\operatorname{ker} \mathcal{D}_{\Lambda}$ on $\Lambda$ we will use the fact that every element $\xi \in \operatorname{ker} \mathcal{D}_{\Lambda}$ is a smooth function from $\mathbb{R}$ to $\operatorname{dom} D_{\Lambda}$ (see [26, Theorem 3.13]) 
and satisfies an estimate of the form $\|\xi\|_{\mathcal{W}_{\Lambda}}+\left\|\partial_{s} \xi\right\|_{\mathcal{W}_{\Lambda}} \leq c\|\xi\|_{\mathcal{H}}$. Two Lagrangian subspaces $\Lambda, \Lambda^{\prime} \in \Upsilon$ are close if there exists an isomorphism of $V$ close to the identity that maps $\Lambda$ to $\Lambda^{\prime}$. This extends to an isomorphism of $\operatorname{dom} D_{\Lambda}=\Lambda \oplus \operatorname{dom} D$ and $\operatorname{dom} D_{\Lambda^{\prime}}=\Lambda^{\prime} \oplus \operatorname{dom} D$ (which does not necessarily extend to an isomorphism of $H$ ). This isomorphism of domains followed by the orthogonal projection onto the kernel of $\mathcal{D}_{\Lambda^{\prime}}$ induces a map $\operatorname{ker} \mathcal{D}_{\Lambda} \rightarrow \mathcal{W}_{\Lambda^{\prime}}$, which is an isomorphism for $\Lambda^{\prime}$ sufficiently close to $\Lambda$.

\section{Appendix C Unique continuation}

In this appendix we formulate a general unique continuation theorem based on the Agmon-Nirenberg technique. The method was also used by Donaldson-Kronheimer [11, pp150] and Taubes [31] to prove unique continuation results for anti-self-dual instantons and by Kronheimer-Mrowka [20] and in [27] for the Seiberg-Witten equations.

Let $H$ be a Hilbert space and $A(s)$ be a family of (unbounded) symmetric operators on $H$ with domains $\operatorname{dom}(A(s)) \subset H$. The operators $A(s)$ are not required to be self-adjoint although in the main applications they will be and, moreover, their domains will be independent of $s$. However, in some interesting cases these operators are symmetric with respect to time-dependent inner products. The following theorem is a special case of a result by Agmon and Nirenberg [2].

Theorem C.1 (Agmon-Nirenberg) Let $H$ be a real Hilbert space and consider a family of symmetric linear operators $A(s): \operatorname{dom}(A(s)) \rightarrow H$. Assume that $x:[0, T) \rightarrow$ $H$ for $0<T \leq \infty$ is continuously differentiable in the weak topology such that $x(s) \in \operatorname{dom}(A(s))$ and

$$
\|\dot{x}(s)+A(s) x(s)\| \leq c_{1}(s)\|x(s)\|
$$

for every $s \in[0, T)$, where $\dot{x}(s):=\partial_{s} x(s) \in H$ denotes the time derivative of $x$. Assume further that the function $s \mapsto\langle x(s), A(s) x(s)\rangle$ is also continuously differentiable and satisfies

$$
\frac{d}{d s}\langle x, A x\rangle-2\langle\dot{x}, A x\rangle \leq 2 c_{2}(s)\|A x\|\|x\|+c_{3}(s)\|x\|^{2} .
$$

Here $c_{1}, c_{2}, c_{3}:[0, T) \rightarrow \mathbb{R}$ are continuous nonnegative functions satisfying

$$
a_{0}:=2 \int_{0}^{T} c_{2}<\infty, \quad b_{0}:=\int_{0}^{T}\left(c_{1}^{2}+c_{2}^{2}+c_{3}\right)<\infty, \quad c_{0}:=\sup c_{1}<\infty .
$$

Then the following holds. 
(i) If $x(0)=0$ then $x(s)=0$ for all $s \in[0, T)$.

(ii) If $x(0) \neq 0$ then $x(s) \neq 0$ for all $s \in[0, T)$ and, moreover,

$$
\|x(s)\| \geq e^{-c s}\|x(0)\|, \quad c:=c_{0}+e^{a_{0}}\left(b_{0}+\|x(0)\|^{-1}\|A(0) x(0)\|\right) .
$$

Proof The basic idea of the proof is to use the convexity of the function $t \mapsto$ $\log \|x(t)\|^{2}$. Assume that $x(0) \neq 0$ and define

$$
\varphi(s):=\log \|x(s)\|-\int_{0}^{s} \frac{\langle x(\sigma), \dot{x}(\sigma)+A(\sigma) x(\sigma)\rangle}{\|x(\sigma)\|^{2}} \mathrm{~d} \sigma
$$

for $0 \leq s<T$ wherever $x(s) \neq 0$. Then we prove that $\varphi$ is twice continuously differentiable and satisfies the differential inequality

$$
\ddot{\varphi}+a|\dot{\varphi}|+b \geq 0, \quad a:=2 c_{2}, \quad b:=c_{1}^{2}+c_{2}^{2}+c_{3} .
$$

Define $f(s):=\dot{x}(s)+A(s) x(s)$. Then the derivative of $\varphi$ is given by

$$
\dot{\varphi}=\frac{\langle x, \dot{x}\rangle}{\|x\|^{2}}-\frac{\langle x, f\rangle}{\|x\|^{2}}=-\frac{\langle x, A x\rangle}{\|x\|^{2}} .
$$

Hence

$$
\begin{aligned}
\ddot{\varphi} & =-\frac{\frac{d}{d s}\langle A x, x\rangle}{\|x\|^{2}}+\frac{2\langle A x, x\rangle\langle\dot{x}, x\rangle}{\|x\|^{4}} \\
& \geq \frac{2\langle A x, A x-f\rangle-2 c_{2}\|A x\|\|x\|-c_{3}\|x\|^{2}}{\|x\|^{2}}-\frac{2\langle A x, x\rangle\langle A x-f, x\rangle}{\|x\|^{4}} .
\end{aligned}
$$

Here the second step follows from the inequality (127) and the definition of $f$. The terms on the right hand side can now be organized as follows

$$
\begin{aligned}
\ddot{\varphi} \geq & \frac{2}{\|x\|^{2}}\left(\|A x\|^{2}-\frac{\langle A x, x\rangle^{2}}{\|x\|^{2}}\right)-\frac{2}{\|x\|^{2}}\left\langle A x-\frac{\langle A x, x\rangle}{\|x\|^{2}} x, f\right\rangle \\
& -2 c_{2} \frac{\|A x\|}{\|x\|}-c_{3} . \\
= & \frac{2}{\|x\|^{2}}\left\|A x-\frac{\langle A x, x\rangle}{\|x\|^{2}} x\right\|^{2}-\frac{2}{\|x\|^{2}}\left\langle A x-\frac{\langle A x, x\rangle}{\|x\|^{2}} x, f\right\rangle \\
& -2 c_{2} \frac{\|A x\|}{\|x\|}-c_{3} .
\end{aligned}
$$

Now abbreviate

$$
\xi=\frac{x}{\|x\|}, \quad \eta=\frac{A x}{\|x\|}
$$

Geometry 83 Topology, Volume 12 (2008) 
Then $\dot{\varphi}=-\langle\xi, \eta\rangle$ and the previous inequality can be written in the form

$$
\begin{aligned}
\ddot{\varphi} & \geq 2\|\eta-\langle\eta, \xi\rangle \xi\|^{2}-2\left\langle\eta-\langle\eta, \xi\rangle \xi, \frac{f}{\|x\|}\right\rangle-2 c_{2}\|\eta\|-c_{3} \\
& \geq 2\|\eta-\langle\eta, \xi\rangle \xi\|^{2}-2\|\eta-\langle\eta, \xi\rangle \xi\| \frac{\|f\|}{\|x\|}-2 c_{2}\|\eta\|-c_{3} \\
& \geq\|\eta-\langle\eta, \xi\rangle \xi\|^{2}-\frac{\|f\|^{2}}{\|x\|^{2}}-2 c_{2}\|\eta\|-c_{3} \\
& \geq\|\eta-\langle\eta, \xi\rangle \xi\|^{2}-c_{1}^{2}-2 c_{2}\|\eta\|-c_{3} .
\end{aligned}
$$

The last but one inequality uses the fact that $\alpha \beta \leq \alpha^{2} / 2+\beta^{2} / 2$ and the last inequality uses $\|f\| \leq c_{1}\|x\|$. To obtain (128) it remains to prove that

$$
\|\eta-\langle\eta, \xi\rangle \xi\|^{2}-2 c_{2}\|\eta\| \geq-2 c_{2}|\dot{\varphi}|-c_{2}^{2} .
$$

Since $\dot{\varphi}=-\langle\xi, \eta\rangle$ this is equivalent to

$$
2 c_{2}\|\eta\| \leq\|\eta-\langle\eta, \xi\rangle \xi\|^{2}+2 c_{2}|\langle\eta, \xi\rangle|+c_{2}^{2} .
$$

Now the norm squared of $\eta$ can be expressed in the form

$$
\|\eta\|^{2}=u^{2}+v^{2}, \quad u=\|\eta-\langle\eta, \xi\rangle \xi\|, \quad v=|\langle\eta, \xi\rangle|
$$

Hence the desired inequality has the form

$$
2 c_{2} \sqrt{u^{2}+v^{2}} \leq u^{2}+2 c_{2} v+c_{2}^{2} .
$$

This follows from the inequalities $\sqrt{u^{2}+v^{2}} \leq u+v$ and $2 c_{2} u \leq u^{2}+c_{2}{ }^{2}$. Thus we have proved (128).

Define $\alpha(s):=\int_{0}^{s} a(\sigma) \mathrm{d} \sigma$. Then $\alpha$ is nonnegative and $\dot{\alpha}=a$. Hence at each point $s \in[0, T)$ with $\dot{\varphi}(s) \leq 0$ we have

$$
\frac{\mathrm{d}}{\mathrm{d} s}\left(e^{-\alpha} \dot{\varphi}\right)=e^{-\alpha}(\ddot{\varphi}+a|\dot{\varphi}|) \geq-b .
$$

Integrating this inequality over maximal intervals where $\dot{\varphi}$ is negative we obtain

$$
e^{-\alpha(s)} \dot{\varphi}(s) \geq \min \{0, \dot{\varphi}(0)\}-\int_{0}^{s} b(\sigma) \mathrm{d} \sigma, \quad \text { for } 0 \leq s<T .
$$

This implies $\dot{\varphi}(s) \geq-e^{a_{0}}\left(b_{0}+|\dot{\varphi}(0)|\right)$, hence $\varphi(s) \geq \varphi(0)-e^{a_{0}}\left(b_{0}+|\dot{\varphi}(0)|\right) s$, and hence, again for $0 \leq s<T$,

$$
\log \|x(s)\| \geq \varphi(s)-\int_{0}^{s}\|x\|^{-1}\|\dot{x}+A x\| \geq \varphi(0)-e^{a_{0}}\left(b_{0}+|\dot{\varphi}(0)|\right) s-c_{0} s .
$$


Now we can use $\log \|x(0)\|=\varphi(0)$ and $|\dot{\varphi}(0)| \leq\|x(0)\|^{-1}\|A(0) x(0)\|$ to prove (ii):

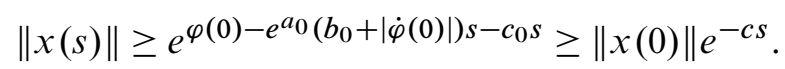

To prove (i) we assume by contradiction that $x\left(s_{0}\right) \neq 0$ for some $s_{0} \in(0, T)$. Then part (ii) applies to the path $s \mapsto x\left(s_{0}-s\right)$ and the operator family $s \mapsto-A\left(s_{0}-s\right)$. It implies $\|x(\sigma)\| \geq e^{c \sigma-c s_{0}}\left\|x\left(s_{0}\right)\right\|$ for all $\sigma \in\left(0, s_{0}\right]$, so by continuity $\|x(0)\| \geq$ $e^{-c s_{0}}\left\|x\left(s_{0}\right)\right\| \neq 0$ in contradiction to the assumption.

\section{Time-dependent inner products}

There are interesting applications to operator families $A(s)$ on a Hilbert space which are self-adjoint with respect to a time-dependent family of inner products which are all compatible with the standard inner product on $H$. Any such family of inner products can be expressed in the form

$$
\langle x, y\rangle_{s}=\langle Q(s) x, Q(s) y\rangle
$$

for some invertible bounded linear operators $Q(s): H \rightarrow H$. Without loss of generality one can consider operators $Q(s)$ which are self-adjoint. Assume throughout that these operators satisfy the following conditions.

(Q1) The operator $Q(s)$ is self-adjoint for every $s$ and there exists a constant $\delta>0$ such that for all $x \in H$ and $s \in[0, T)$

$$
\delta\|x\| \leq\|Q(s) x\| \leq \delta^{-1}\|x\| .
$$

Moreover, the map $[0, T) \rightarrow \mathcal{L}(H): s \mapsto Q(s)$ is continuously differentiable in the weak operator topology and there exists a continuous function $c_{Q}:[0, T) \rightarrow$ $[0, \infty)$ such that

$$
\|\dot{Q}(s)\|_{\mathcal{L}(H)} \leq c_{Q}(s) \quad \forall s \in[0, T), \quad C_{Q}:=\int_{0}^{T} c_{Q}<\infty .
$$

Theorem C.2 Let $H$ be a real Hilbert space, $Q(s) \in \mathcal{L}(H)$ a family of (bounded) selfadjoint operators satisfying $(Q 1)$, and $A(s)$ : $\operatorname{dom}(A(s)) \rightarrow H$ a family of (unbounded) linear operators such that $A(s)$ is symmetric with respect to the inner product (129). Assume that $x:[0, T) \rightarrow H$ is continuously differentiable in the weak topology such that $x(s) \in \operatorname{dom}(A(s))$ and

$$
\|\dot{x}(s)+A(s) x(s)\|_{s} \leq c_{1}(s)\|x(s)\|_{s}
$$


for every $s \in[0, T)$. Assume further that the function $s \mapsto\langle x(s), A(s) x(s)\rangle_{s}$ is also continuously differentiable and satisfies

$$
\begin{aligned}
\frac{\mathrm{d}}{\mathrm{d} s}\langle x(s), A(s) x(s)\rangle_{s}-2\langle\dot{x}(s), A(s) & x(s)\rangle_{s} \\
& \leq 2 c_{2}(s)\|A(s) x(s)\|_{s}\|x(s)\|_{s}+c_{3}(s)\|x(s)\|_{s}^{2}
\end{aligned}
$$

for every $s \in[0, T)$. Here $c_{1}, c_{2}, c_{3}:[0, T) \rightarrow \mathbb{R}$ are continuous nonnegative functions satisfying

$$
\begin{aligned}
a_{0} & :=2 \int_{0}^{T}\left(c_{2}+\delta^{-1} c_{Q}\right)<\infty, \\
b_{0} & :=\int_{0}^{T}\left(\left(c_{1}+\delta^{-1} c_{Q}\right)^{2}+\left(c_{2}+\delta^{-1} c_{Q}\right)^{2}+c_{3}\right)<\infty, \\
c_{0} & :=\sup \left(c_{1}+\delta^{-1} c_{Q}\right)<\infty .
\end{aligned}
$$

Then the following holds.

(i) If $x(0)=0$ then $x(s)=0$ for all $s \in[0, T)$.

(ii) If $x(0) \neq 0$ then $x(s) \neq 0$ for all $s \in[0, T)$ and, moreover,

$$
\|x(s)\|_{s} \geq e^{-c s}\|x(0)\|_{0}, \quad c:=c_{0}+e^{a_{0}}\left(b_{0}+\|x(0)\|_{0}^{-1}\|A(0) x(0)\|_{0}\right) .
$$

Proof The result reduces to Theorem C.1. Define

$$
\tilde{A}:=Q A Q^{-1}, \quad \tilde{x}:=Q x, \quad \tilde{f}:=\dot{Q} x+Q f
$$

with $\operatorname{dom}(\tilde{A}(s))=Q(s) \operatorname{dom}(A(s))$ and $f=\dot{x}+A x$. Then the operator $A(s)$ is symmetric with respect to the inner product (129) if and only if $\widetilde{A}(s)$ is symmetric with respect to the standard inner product. (Moreover, one can easily check that $A(s)$ is self-adjoint with respect to (129) if and only if $\widetilde{A}(s)$ is self-adjoint with respect to the standard inner product. However, this is not needed for the proof.) It also easy to see that

$$
\dot{x}+A x=f \quad \Longleftrightarrow \quad \dot{\tilde{x}}+\tilde{A} \tilde{x}=\tilde{f} .
$$

It remains to show that under the assumptions of Theorem C.2 the triple $\tilde{A}, \tilde{x}, \tilde{f}$ satisfies the requirements of Theorem C.1. First, note that

$$
\|\tilde{f}\|=\|\dot{Q} x+Q f\| \leq c_{Q}\|x\|+\|f\|_{s} \leq c_{Q} \delta^{-1}\|x\|_{s}+c_{1}\|x\|_{s}
$$

and hence $\tilde{x}$ satisfies (126) with $c_{1}$ replaced by $\tilde{c}_{1}=c_{1}+c_{Q} / \delta$. Secondly, the function

$$
s \mapsto\langle\tilde{x}(s), \tilde{A}(s) \tilde{x}(s)\rangle=\langle x(s), A(s) x(s)\rangle_{s}
$$


is continuously differentiable and a simple calculation shows that

$$
\frac{\mathrm{d}}{\mathrm{d} s}\langle\tilde{x}, \tilde{A} \tilde{x}\rangle-2\langle\dot{\tilde{x}}, \tilde{A} \tilde{x}\rangle=\frac{\mathrm{d}}{\mathrm{d} s}\langle x, A x\rangle_{s}-2\langle\dot{x}, A x\rangle_{s}-2\langle\dot{Q} x, Q A x\rangle .
$$

Hence

$$
\begin{aligned}
\frac{\mathrm{d}}{\mathrm{d} s}\langle\tilde{x}, \tilde{A} \tilde{x}\rangle-2\langle\dot{\tilde{x}}, \tilde{A} \tilde{x}\rangle & \leq 2 c_{2}\|x\|_{s}\|A x\|_{s}+c_{3}\|x\|_{s}^{2}+2\|\dot{Q} x\|\|\tilde{A} \tilde{x}\| \\
& \leq 2 c_{2}\|\tilde{x}\|\|\tilde{A} \tilde{x}\|+c_{3}\|\tilde{x}\|^{2}+2 c_{Q} \delta^{-1}\|\tilde{x}\|\|\tilde{A} \tilde{x}\| .
\end{aligned}
$$

This shows that $\tilde{x}$ satisfies (127) with $c_{2}$ and $c_{3}$ replaced by $\tilde{c}_{2}=c_{2}+c_{Q} / \delta$ and $\tilde{c}_{3}=c_{3}$. Hence $\tilde{x}$ and $\tilde{A}$ satisfy the requirements of Theorem C. 1 and this proves Theorem C.2.

\section{Appendix D Holonomy perturbations}

In this appendix we review the properties of the holonomy perturbations used in this paper. Throughout this appendix $Y$ is a compact oriented 3-manifold, $\mathbb{D} \subset \mathbb{C}$ is the closed unit disc, and we identify the circle $S^{1}$ with $\mathbb{R} / \mathbb{Z}$. The elements of $S^{1}$ will be denoted by $\theta$ and those of $\mathbb{D}$ by $z$. Fix a finite sequence of orientation preserving embeddings $\gamma_{i}: S^{1} \times \mathbb{D} \rightarrow Y$ for $i=1, \ldots, N$ that coincide in a neighbourhood of $\{0\} \times \mathbb{D}$. Define the holonomy maps

$$
g_{i}: \mathbb{R} \times \mathbb{D} \times \mathcal{A}(Y) \rightarrow \mathrm{G}, \quad \rho_{i}: \mathbb{D} \times \mathcal{A}(Y) \rightarrow \mathrm{G}
$$

by $\quad \partial_{\theta} g_{i}+A\left(\partial_{\theta} \gamma_{i}\right) g_{i}=0, \quad g_{i}(0, z ; A)=\mathbb{1}, \quad \rho_{i}(z ; A):=g_{i}(1, z ; A)$.

and abbreviate $\rho:=\left(\rho_{1}, \ldots, \rho_{N}\right): \mathbb{D} \times \mathcal{A}(Y) \rightarrow \mathrm{G}^{N}$. Fix a smooth conjugation invariant function $f: \mathbb{D} \times \mathrm{G}^{N} \rightarrow \mathbb{R}$ that vanishes near the boundary, and define the perturbation $h_{f}: \mathcal{A}(Y) \rightarrow \mathbb{R}$ by

$$
h_{f}(A):=\int_{\mathbb{D}} f(z, \rho(z ; A)) \mathrm{d}^{2} z .
$$

This map is smooth and its derivative has the form

$$
\mathrm{d} h_{f}(A) \alpha=\left.\frac{\mathrm{d}}{\mathrm{d} s}\right|_{s=0} \int_{\mathbb{D}} f(z, \rho(z ; A+s \alpha)) \mathrm{d}^{2} z=\int_{Y}\left\langle X_{f}(A) \wedge \alpha\right\rangle
$$

for $\alpha \in \Omega^{1}(Y, \mathfrak{g})$. The map $X_{f}: \mathcal{A}(Y) \rightarrow \Omega^{2}(Y, \mathfrak{g})$ is uniquely determined by (130); it has the form

$$
X_{f}(A)=\sum_{i=1}^{N} \gamma_{i *}\left(X_{f, i}(A) \mathrm{d}^{2} z\right),
$$


where $X_{f, i}(A) \in \Omega^{0}\left(S^{1} \times \mathbb{D}, \mathfrak{g}\right)$ is given by

$$
X_{f, i}(A)(\theta, z)=-g_{i}(\theta, z ; A) \nabla_{i} f(z, \rho(z ; A)) g_{i}(\theta, z ; A)^{-1} .
$$

Here the gradient $\nabla_{i} f: \mathbb{D} \times \mathrm{G}^{N} \rightarrow \mathfrak{g}$ is defined by

$$
\left\langle\nabla_{i} f(z, g), \xi\right\rangle:=\left.\frac{\mathrm{d}}{\mathrm{d} t}\right|_{t=0} f\left(z, g_{1}, \ldots, g_{i-1}, g_{i} \exp (t \xi), g_{i+1}, \ldots, g_{N}\right)
$$

for $z \in \mathbb{D}, g=\left(g_{1}, \ldots, g_{N}\right) \in \mathrm{G}^{N}$, and $\xi \in \mathfrak{g}$. It vanishes near the boundary of $\mathbb{D}$ and, since $f$ is conjugation invariant, it satisfies

$$
\nabla_{i} f\left(z, h g h^{-1}\right)=h \nabla_{i} f(z, g) h^{-1}
$$

for $h \in \mathrm{G}$. If follows from (132) that $X_{f, i}(A): \mathbb{R} \times \mathbb{D} \rightarrow \mathfrak{g}$ descends to a function on $S^{1} \times$ $\mathbb{D}$. If the center of $\mathrm{G}$ is discrete then Equation (132) implies that $\nabla_{i} f(z,(\mathbb{1}, \ldots, \mathbb{1}))=0$ and hence $X_{f}(0)=0$ for every $f \in C_{0}^{\infty}\left(\mathbb{D} \times \mathrm{G}^{N}\right)^{\mathrm{G}}$. Thus, for $\mathrm{G}=\mathrm{SU}(2)$ the trivial connection is always a critical point of the perturbed Chern-Simons functional $\mathcal{C} \mathcal{S}_{\mathcal{L}}+h_{f}$. The next proposition summarizes the properties of $X_{f}$. We denote the space connections of class $W^{k, p}$ by

$$
\mathcal{A}^{k, p}(Y):=W^{k, p}\left(Y, \mathrm{~T}^{*} Y \otimes \mathfrak{g}\right) .
$$

Proposition D.1 Let $f \in \mathcal{C}_{0}^{\ell+1}\left(\mathbb{D} \times \mathrm{G}^{N}\right)^{\mathrm{G}}$ for some integer $\ell \geq 0$. Then the following holds (with uniform constants independent of $f$ ).

(i) For every integer $\ell \geq k \geq 1$ and every $p>2$ with $k p>3, X_{f}$ extends to a $\mathcal{C}^{\ell-k}$ map from $\mathcal{A}^{k, p}(Y)$ to $W^{k, p}\left(Y, \Lambda^{2} \mathrm{~T} Y \otimes \mathfrak{g}\right)$, mapping bounded sets to bounded sets.

(ii) For all $A \in \mathcal{A}(Y), u \in \mathcal{G}(Y), \xi \in \Omega^{0}(Y, \mathfrak{g})$, and $\alpha \in \Omega^{1}(Y, \mathfrak{g})$ we have

$$
\begin{gathered}
\mathrm{d}_{A}\left(X_{f}(A)\right)=0, \quad X_{f}\left(u^{*} A\right)=u^{-1} X_{f}(A) u, \\
\mathrm{~d} X_{f}(A) \mathrm{d}_{A} \xi=\left[X_{f}(A), \xi\right], \quad \mathrm{d}_{A}\left(\mathrm{~d} X_{f}(A) \alpha\right)=\left[X_{f}(A) \wedge \alpha\right] .
\end{gathered}
$$

(iii) For every $k \in\{0, \ldots, \ell\}$ and every $p \in[1, \infty]$ there is a constant $c$ such that

$$
\left\|X_{f}(A)\right\|_{W^{k, p}} \leq c\|\nabla f\|_{\mathcal{C}^{k}}\left(1+\sum_{\substack{j_{0}+\cdots+j_{s}=k \\ s \geq 0, j_{\nu} \geq 1}}\|A\|_{W^{j_{0}, p}}\|A\|_{\mathcal{C}^{j_{1}}} \cdots\|A\|_{\mathcal{C}^{j_{s}}}\right)
$$

for every $A \in \mathcal{A}(Y)$. If $k=0$ then $\left\|X_{f}(A)\right\|_{L^{p}} \leq c\|\nabla f\|_{L^{p}}$.

(iv) For every $k \in\{0, \ldots, \ell-1\}$ and $p \in[1, \infty]$ there is a constant $c$ such that

$$
\left\|\mathrm{d} X_{f}(A) \alpha\right\|_{W^{k, p}(Y)} \leq c\|\nabla f\|_{\mathcal{C}^{k+1}}\left(1+\|A\|_{\mathcal{C}^{k}}\right)^{k}\|\alpha\|_{W^{k, p}(Y)}
$$

for all $A \in \mathcal{A}(Y)$ and $\alpha \in \Omega^{1}(Y, \mathfrak{g})$. 
(v) For all $p, q, r \in[1, \infty]$ with $q^{-1}+r^{-1}=p^{-1}$ there is a constant $c$ such that

$$
\begin{aligned}
& \left\|\mathrm{d}^{2} X_{f}(A)(\alpha, \beta)\right\|_{L^{p}(Y)} \leq c\|\nabla f\|_{\mathcal{C}^{1}}\|\alpha\|_{L^{q}(Y)}\|\beta\|_{L^{r}(Y)}, \\
& \left\|X_{f}(A+\alpha)-X_{f}(A)-\mathrm{d} X_{f}(A) \alpha\right\|_{L^{p}(Y)} \leq c\|\nabla f\|_{\mathcal{C}^{1}}\|\alpha\|_{L^{q}(Y)}\|\alpha\|_{L^{r}(Y)}
\end{aligned}
$$

for all $A \in \mathcal{A}(Y)$ and $\alpha, \beta \in \Omega^{1}(Y, \mathfrak{g})$.

(vi) For every $p \in[1, \infty]$ there is a uniform constant $c$ such that

$$
\left\|\nabla_{A}\left(X_{f}(A)\right)\right\|_{L^{p}} \leq c\left(1+\|\nabla f\|_{\mathcal{C}^{1}}\right)\left(1+\left\|F_{A}\right\|_{L^{p}}\right)
$$

for every $A \in \mathcal{A}(Y)$.

Remark D.2 Consider a connection $\Xi=\Phi \mathrm{d} s+A \in \mathcal{A}(I \times Y)$ for a compact interval $I$, given by $A: I \rightarrow \mathcal{A}(Y)$ and $\Phi: I \rightarrow \Omega^{0}(Y, \mathfrak{g})$. Proposition D.1 extends to the perturbation $X_{f}(\Xi):=X_{f} \circ A \in \Omega^{2}(I \times Y, \mathfrak{g})$ - except for (ii), and in (i) we need to assume $k p>4$. In particular, for every $k \geq 1$ and $p>2, X_{f}$ maps bounded sets in $\mathcal{A}^{k, p}(I \times Y)$ to bounded sets in $W^{k, p}\left(I \times Y, \Lambda^{2} \mathrm{~T} Y \otimes \mathfrak{g}\right)$. In the case $k=1$ and $k p \leq 4$ this follows from Proposition D.1 (iii).

The proof requires some preparation. We begin by considering connections on the circle. The canonical 1 -form $d \theta \in \Omega^{1}\left(S^{1}\right)$ allows us to identify the space $\mathcal{A}\left(S^{1}\right)=\Omega^{1}\left(S^{1}, \mathfrak{g}\right)$ of G-connections on $S^{1}$ with the space $\Omega^{0}\left(S^{1}, \mathfrak{g}\right)$ of Lie algebra valued functions. The holonomy of a connection $A=\eta \mathrm{d} \theta \in \mathcal{A}\left(S^{1}\right)$ with $\eta: S^{1} \rightarrow \mathfrak{g}$ is the solution $g: \mathbb{R} \rightarrow \mathrm{G}$ of the differential equation

$$
\partial_{\theta} g+\eta g=0, \quad g(0)=11 .
$$

The solutions give rise to a map hol: $\mathbb{R} \times \Omega^{0}\left(S^{1}, \mathfrak{g}\right) \rightarrow \mathrm{G}$ which assigns to each pair $(\theta, \eta) \in \mathbb{R} \times \Omega^{0}\left(S^{1}, \mathfrak{g}\right)$ the value $\operatorname{hol}(\theta ; \eta):=g(\theta)$ of the unique solution of (134) at $\theta$. The gauge invariance of the holonomy takes the form

$$
\operatorname{hol}\left(\theta ; u^{-1} \partial_{\theta} u+u^{-1} \eta u\right)=u(\theta)^{-1} \operatorname{hol}(\theta ; \eta) u(0)
$$

for $u: S^{1} \rightarrow \mathrm{G}$. One can think of hol as a map from $\Omega^{0}\left(S^{1}, \mathfrak{g}\right)$ to $C^{\infty}([0,1], \mathrm{G})$ defined by $\operatorname{hol}(\eta)(\theta):=\operatorname{hol}(\theta ; \eta)$. The holonomy then induces a map between Sobolev completions, for every integer $k \geq 0$ and every $p \geq 1$,

$$
\text { hol: } W^{k, p}\left(S^{1}, \mathfrak{g}\right) \rightarrow W^{k+1, p}([0,1], \mathrm{G}) .
$$

This map is continuously differentiable and its derivative at $\eta \in W^{k, p}\left(S^{1}, \mathfrak{g}\right)$ is the bounded linear operator $\operatorname{d} \operatorname{hol}(\eta): W^{k, p}\left(S^{1}, \mathfrak{g}\right) \rightarrow W^{k+1, p}\left([0,1], \operatorname{hol}(\eta)^{*} \mathrm{TG}\right)$ given 
by

$$
\left(\operatorname{hol}(\eta)^{-1} \mathrm{~d} \operatorname{hol}(\eta) \hat{\eta}\right)(\theta)=-\int_{0}^{\theta} \operatorname{hol}(t ; \eta)^{-1} \hat{\eta}(t) \operatorname{hol}(t ; \eta) \mathrm{d} t
$$

for $\hat{\eta} \in W^{k, p}\left(S^{1}, \mathfrak{g}\right)$. The formula (136) shows, by induction, that the map (135) is smooth. The next lemma is a parametrized version of this observation.

Lemma D.3 Let $\Omega$ be a compact Riemannian manifold.

(i) For every integer $k \geq 1$ and every $p>\operatorname{dim} \Omega$, composition with the holonomy induces smooth maps

$$
\begin{aligned}
& \text { Hol: } W^{k, p}\left(S^{1} \times \Omega, \mathfrak{g}\right) \rightarrow W^{k, p}([0,1] \times \Omega, \mathrm{G}), \\
& \mathrm{Hol}_{1}: W^{k, p}\left(S^{1} \times \Omega, \mathfrak{g}\right) \rightarrow W^{k, p}(\Omega, \mathrm{G}),
\end{aligned}
$$

given by $\operatorname{Hol}(\eta):=g_{\eta}$ with $g_{\eta}(\theta, x):=\operatorname{hol}(\theta, \eta(\cdot, x))$ and $\operatorname{Hol}_{1}(\eta)(x):=g_{\eta}(1, x)$ for $x \in \Omega$ and $\theta \in[0,1]$. These map $W^{k, p}$-bounded sets to $W^{k, p}$-bounded sets.

(ii) For every integer $k \geq 1$ there is a constant $c$ such that

$$
\|\operatorname{Hol}(\eta)\|_{W^{k, p}}+\left\|\operatorname{Hol}_{1}(\eta)\right\|_{W^{k, p}} \leq c\left(1+\sum_{\substack{j_{0}+\cdots+j_{s}=k \\ s \geq 0, j_{\nu} \geq 1}}\|\eta\|_{W^{j_{0}, p}}\|\eta\|_{\mathcal{C}^{j_{1}}} \cdots\|\eta\|_{\mathcal{C}^{j_{s}}}\right)
$$

for every $\eta \in \mathcal{C}^{k}\left(S^{1} \times \Omega, \mathfrak{g}\right)$ and every $p \in[1, \infty]$.

(iii) For every integer $k \geq 0$ and every $p \in[1, \infty]$ there is a uniform constant $c$ such that, for every $\eta \in C^{k}\left(S^{1} \times \Omega, \mathfrak{g}\right)$, the derivatives

$$
\begin{aligned}
& \operatorname{Hol}(\eta)^{-1} \mathrm{dHol}(\eta): W^{k, p}\left(S^{1} \times \Omega, \mathfrak{g}\right) \rightarrow W^{k, p}([0,1] \times \Omega, \mathfrak{g}), \\
& \operatorname{Hol}_{1}(\eta)^{-1} \mathrm{dHol}_{1}(\eta): W^{k, p}\left(S^{1} \times \Omega, \mathfrak{g}\right) \rightarrow W^{k, p}(\Omega, \mathfrak{g})
\end{aligned}
$$

are bounded linear operators with norms less than or equal to $c\left(1+\|\eta\|_{\mathcal{C}^{k}}\right)^{k}$.

Proof Think of $\eta$ as a map from $\Omega$ to $W^{j, p}\left(S^{1}, \mathfrak{g}\right)$ and of $\operatorname{Hol}(\eta)$ as a map from $\Omega$ to $W^{j+1, p}([0,1], \mathrm{G})$. Then $\operatorname{Hol}(\eta)$ is the composition

$$
\Omega \stackrel{\eta}{\longrightarrow} W^{j, p}\left(S^{1}, \mathfrak{g}\right) \stackrel{\text { hol }}{\longrightarrow} W^{j+1, p}([0,1], \mathrm{G}) .
$$

Since hol: $W^{j, p}\left(S^{1}, \mathfrak{g}\right) \rightarrow W^{j+1, p}([0,1], \mathrm{G})$ is smooth the composition induces a smooth map

$$
\text { Hol: } W^{\ell, p}\left(\Omega, W^{j, p}\left(S^{1}, \mathfrak{g}\right)\right) \rightarrow W^{\ell, p}\left(\Omega, W^{j+1, p}([0,1], \mathrm{G})\right)
$$


for $\ell p>\operatorname{dim} \Omega$ and any $j$; hence it defines a smooth map from

$$
\begin{gathered}
W^{k, p}\left(S^{1} \times \Omega, \mathfrak{g}\right)=\bigcap_{\ell=0}^{k} W^{\ell, p}\left(\Omega, W^{k-\ell, p}\left(S^{1}, \mathfrak{g}\right)\right) \\
\bigcap_{\ell=1}^{k} W^{\ell, p}\left(\Omega, W^{k-\ell+1, p}([0,1], \mathrm{G})\right) \subset W^{k, p}([0,1] \times \Omega, \mathrm{G})
\end{gathered}
$$

for $k \geq 1$ and $p>\operatorname{dim} \Omega$. This proves (i) for Hol. To prove (i) for $\mathrm{Hol}_{1}$, take $\ell=k$ and note that evaluation at $\theta=1$ gives a smooth map from $W^{k, p}\left(\Omega, W^{1, p}([0,1], \mathrm{G})\right)$ to $W^{k, p}(\Omega, \mathrm{G})$. The boundedness of Hol and $\mathrm{Hol}_{1}$ is a consequence of (ii) and (iii).

To prove (ii) we differentiate the function $g(\theta, x)=\operatorname{hol}(\theta, \eta(\cdot, x))$ :

$$
g^{-1} \partial_{\theta} g=-g^{-1} \eta g, \quad\left(g^{-1} \partial_{x} g\right)(\theta, x)=-\int_{0}^{\theta} g(t, x)^{-1} \partial_{x} \eta(t, x) g(t, x) \mathrm{d} t .
$$

Hence there are constants $c_{1}, c_{2}, c_{3}, \ldots$ such that

$$
\|g\|_{W^{k, p}} \leq c_{k}\left(1+\sum_{\substack{j_{0}+\ldots+j_{s}=k \\ s \geq 0, j_{v} \geq 1}}\|\eta\|_{W^{j_{0}, p}}\|g\|_{\mathcal{C}^{j_{1}}} \cdots\|g\|_{\mathcal{C}^{j_{s}}}\right)
$$

for every smooth function $\eta: S^{1} \times \Omega \rightarrow \mathfrak{g}$, every integer $k \geq 1$, and every $p \in[1, \infty]$. For $p=\infty$ assertion (ii) now follows by induction on $k$. Inserting the resulting estimate into (137) proves (ii) for all $p$. For $k=0$ assertion (iii) follows immediately from (136) with $c=1$. To prove (iii) for $k \geq 1$ differentiate Equation (136) with respect to $\theta$ and $x$ and use (ii). This proves the lemma.

Proof of Proposition D.1 The map $X_{f, i}: \mathcal{A}^{k, p}(Y) \rightarrow W^{k, p}\left(S^{1} \times \mathbb{D}, \mathfrak{g}\right)$ can be expressed as composition of three maps. The first is the product of the $N$ maps

$$
\mathcal{A}^{k, p}(Y) \rightarrow W^{k, p}\left(S^{1} \times \mathbb{D}, \mathfrak{g}\right): A \mapsto \eta_{j}:=A\left(\partial_{\theta} \gamma_{j}\right),
$$

the second is given by composition with the holonomy

$$
W^{k, p}\left(S^{1} \times \mathbb{D}, \mathfrak{g}\right) \rightarrow W^{k, p}\left([0,1] \times \mathbb{D}, \mathrm{G}^{2}\right): \eta_{j} \mapsto\left(g_{j}, \rho_{j}\right),
$$

where $g_{j}(\theta, z):=\operatorname{hol}\left(\eta_{j}(\cdot, z)\right)(\theta)$ and $\rho_{j}(\theta, z):=\operatorname{hol}\left(\eta_{j}(\cdot, z)\right)(1)$, and the third map has the form

$$
\begin{aligned}
W^{k, p}\left([0,1] \times \mathbb{D}, \mathrm{G}^{2 N}\right) & \rightarrow W^{k, p}\left(S^{1} \times \mathbb{D}, \mathfrak{g}\right):\left(g_{1}, \rho_{1}, \ldots, g_{N}, \rho_{N}\right) \mapsto \zeta, \\
\text { with } \quad \zeta & :=g_{i} \nabla_{i} f\left(\rho_{1}, \ldots, \rho_{N}\right) g_{i}^{-1}
\end{aligned}
$$


(see Equation (131)). The first map is bounded linear (and hence smooth) for all $k$ and $p$ because composition with a smooth embedding at the source and multiplication with a smooth function define bounded linear maps between $W^{k, p}$-spaces. The second map is smooth and bounded for $k \geq 1$ and $p>2$ by Lemma D.3. The third map is bounded and $\mathcal{C}^{\ell-k}$ because composition with a $\mathcal{C}^{k}$-map at the target defines a continuous map from $W^{k, p}$ to $W^{k, p}$ for all $k p \geq \operatorname{dim}(\mathbb{R} \times \mathbb{D})\left(\right.$ or $k p \geq \operatorname{dim}\left(\mathbb{R}^{2} \times \mathbb{D}\right)$ in the case of Remark D.2). This proves (i). Assertion (ii) follows by straight forward calculations and (iii) follows from (131) and Lemma D.3 (ii).

To prove (iv) we abbreviate $A_{i}:=\gamma_{i}^{*} A_{i}, \alpha_{i}:=\gamma_{i}^{*} \alpha_{i}$, and differentiate Equation (131) to obtain

$$
\begin{aligned}
\operatorname{Hol}\left(A_{i}\right)^{-1}\left(\mathrm{~d} X_{f, i}(A) \alpha\right) \operatorname{Hol}\left(A_{i}\right) & \\
= & {\left[\operatorname{Hol}\left(A_{i}\right)^{-1} X_{f, i}(A) \operatorname{Hol}\left(A_{i}\right), \operatorname{Hol}\left(A_{i}\right)^{-1} \mathrm{dHol}\left(A_{i}\right) \alpha_{i}\right] } \\
& -\sum_{j=1}^{N} \nabla_{j} \nabla_{i} f\left(\left(\operatorname{Hol}_{1}\left(A_{\ell}\right)\right)_{\ell=1, \ldots N}\right) \operatorname{Hol}_{1}\left(A_{j}\right)^{-1} \mathrm{dHol}_{1}\left(A_{j}\right) \alpha_{j} .
\end{aligned}
$$

The estimate now follows from Lemma D.3 and the uniform bounds in (iii).

To prove (v) we differentiate the last equation again and obtain the inequality

$$
\begin{aligned}
& \begin{aligned}
&\left|\mathrm{d}^{2} X_{f}(A)(\alpha, \beta)\right| \\
& \leq\left|\mathrm{d} X_{f}(A) \alpha\right||\mathrm{dHol}(A) \beta|+\left|\mathrm{d} X_{f}(A) \beta\right||\mathrm{d} \operatorname{Hol}(A) \alpha| \\
&+\left|X_{f}(A)\right||\mathrm{dHol}(A) \alpha||\operatorname{dHol}(A) \beta|+\left|X_{f}(A)\right|\left|\mathrm{d}\left(\operatorname{Hol}(A)^{-1} \mathrm{dHol}(A) \alpha\right) \beta\right| \\
&+\left|\nabla^{2} f\right|\left|\mathrm{dHol}_{1}(A) \alpha\right|\left|\operatorname{dHol}_{1}(A) \beta\right|+\left|\nabla^{2} f\right|\left|\mathrm{d}\left(\operatorname{Hol}_{1}(A)^{-1} \mathrm{dHol}_{1}(A) \alpha\right) \beta\right|
\end{aligned} \\
& \text { with } \quad \mathrm{d}\left(\operatorname{Hol}(A)^{-1} \mathrm{dHol}(A) \alpha\right) \beta=\int_{0}^{\theta}\left[\operatorname{Hol}(A)^{-1} \mathrm{dHol}(A) \alpha, \operatorname{Hol}(A)^{-1} \mathrm{dHol}(A) \beta\right] .
\end{aligned}
$$

A similar inequality holds for $\mathrm{Hol}_{1}$. The first estimate in (v) now follows from the $L^{q}$ and $L^{r}$-bounds in (iv) and Lemma D.3 and the $L^{\infty}$-bounds on $X_{f}$ and $\nabla^{2} f$. The second estimate in (v) follows from the first and

$$
X_{f}(A+\alpha)-X_{f}(A)-\mathrm{d} X_{f}(A) \alpha=\int_{0}^{1} \int_{0}^{\tau} \mathrm{d}^{2} X_{f}(A+t \alpha)(\alpha, \alpha) \mathrm{d} t \mathrm{~d} \tau .
$$

Assertion (vi) is a result of Froyshov [15]. The proof uses the formula

$$
\begin{aligned}
& \partial_{t} g(\theta, t)+A\left(\partial_{t} \gamma(\theta, t)\right) g(\theta, t) \\
& =g(\theta, t)\left(\int_{0}^{\theta} g(s, t)^{-1} F_{A}\left(\partial_{\theta} \gamma(s, t), \partial_{t} \gamma(s, t)\right) g(s, t) d s\right)
\end{aligned}
$$


for $\gamma:[0,1]^{2} \rightarrow Y$ and $g:[0,1]^{2} \rightarrow \mathrm{G}$ with

$$
\partial_{\theta} g+A\left(\partial_{\theta} \gamma\right) g=0, \quad g(0, t)=11 .
$$

Namely, inserting a $t$-dependent parameter $z=z(t)$ into (131), abbreviating

$$
\begin{gathered}
g(\theta, t):=g_{i}(\theta, z(t) ; A), \quad \gamma_{i}(\theta, t):=\gamma_{i}(\theta, z(t)), \\
\xi(\theta, t):=X_{f, i}(\theta, z(t))=-g(\theta, t) \nabla_{i} f(z(t), \rho(z(t) ; A)) g(\theta, t)^{-1},
\end{gathered}
$$

and differentiating $\xi$ covariantly with respect to $\gamma_{i}^{*} A$ we find that $\nabla_{\theta} \xi=0$ and

$$
\begin{aligned}
\nabla_{t} \xi= & \partial_{t} \xi+\left[A\left(\partial_{t} \gamma_{i}\right), \xi\right] \\
= & {\left[\left(\partial_{t} g g^{-1}+A\left(\partial_{t} \gamma_{i}\right)\right), \xi\right]-g^{-1}\left(\left(\partial_{1} \nabla_{i} f\right)(z, \rho(z ; A)) \partial_{t} z\right) g } \\
& -g^{-1}\left(\sum_{j=1}^{N}\left(\nabla_{j} \nabla_{i} f\right)(z, \rho(z ; A)) \rho_{j}(z ; A)^{-1} \partial_{t} \rho_{j}(z ; A)\right) g .
\end{aligned}
$$

Since the estimate (133) is gauge invariant and the $\gamma_{j}$ all coincide near $\gamma_{j}(0, z)=$ $\gamma_{j}(1, z)$ we can assume that $A\left(\partial_{t} \gamma_{j}(1, z(t))\right)=0$ for all $j$ and $t$. Then it follows from (138) that

$$
\rho_{j}(z(t) ; A)^{-1} \partial_{t} \rho_{j}(z(t) ; A)=\int_{0}^{1} g_{j}(s, t)^{-1} F_{A}\left(\partial_{\theta} \gamma_{j}(s, t), \partial_{t} \gamma_{j}(s, t)\right) g_{j}(s, t) d s .
$$

So the first and third term on the right hand side of (139) can be estimated by the curvature of $A$, and the second term is uniformly bounded. This proves the proposition.

In the remainder of this section we give a proof of the basic compactness result for solutions $\Xi \in \mathcal{A}(\mathbb{R} \times Y)$ of the perturbed anti-self-duality equation

$$
\left(F_{\Xi}+X_{f}(\Xi)\right)^{+}=0
$$

with bounded energy

$$
E_{f}(\Xi)=\frac{1}{2} \int_{\mathbb{R} \times Y}\left|F_{\Xi}+X_{f}(\Xi)\right|^{2} .
$$

A similar proof for somewhat different perturbations can be found in [19].

Theorem D.4 There exists a universal constant $\hbar>0$ such that the following holds for every perturbation $X_{f}$, every real number $E>0$, and every $p>1$.

Let $\Xi_{v} \in \mathcal{A}(\mathbb{R} \times Y)$ be a sequence of solutions of (139) with bounded energy

$$
\sup _{v} E_{f}\left(\Xi_{v}\right) \leq E
$$


Then there exists a subsequence (again denoted $\left(\Xi_{v}\right)$ ) and a finite set of bubbling points $S=\left\{x_{1}, \ldots, x_{N}\right\} \subset \mathbb{R} \times \operatorname{int}(Y)$ with

$$
\liminf _{\nu \rightarrow \infty} \frac{1}{2} \int_{B_{\delta}\left(x_{j}\right)}\left|F_{\Xi_{v}}+X_{f}\left(\Xi_{v}\right)\right|^{2} \geq \hbar \quad \forall \delta>0, x_{j} \in S .
$$

Moreover, there is a sequence of gauge transformations $u_{v} \in \mathcal{G}((\mathbb{R} \times \operatorname{int}(Y)) \backslash S)$ and a limit connection $\Xi_{\infty} \in \mathcal{A}(\mathbb{R} \times \operatorname{int}(Y))$ such that $u_{v}^{*} \Xi_{v}$ converges to $\Xi_{\infty}$ in the $W^{1, p}$-norm on every compact subset of $\mathbb{R} \times \operatorname{int}(Y) \backslash S$. The limit $\Xi_{\infty}$ solves (139) and has energy

$$
E_{f}\left(\Xi_{\infty}\right) \leq \limsup _{\nu \rightarrow \infty} E_{f}\left(\Xi_{v}\right)-N \hbar .
$$

Remark D.5 If $S \subset\left(T_{-}, T_{+}\right) \times Y$ in Theorem D.4, then the convergence can be improved to the $\mathcal{C}^{\infty}$-topology on every compact subset of $\left(-\infty, T_{-}\right] \times \operatorname{int}(Y)$ and $\left[T_{+}, \infty\right) \times \operatorname{int}(Y)$ (in particular on $\mathbb{R} \times \operatorname{int}(Y)$ if $S=\varnothing$ ). This follows from the standard bootstrapping techniques (eg [11], [34]) and Remark D.2. The crucial point is that a $W^{k, p}$-bound on $u^{v *} \Xi^{v}$ implies a $W^{k, p}$-bound on $X_{f}\left(u^{v^{*}} \Xi^{v}\right)$ and thus on $F_{u^{\nu} *}^{+} \Xi^{\nu}$. The appropriate gauge transformations can be interpolated to the ones over $\left(T_{-}, T_{+}\right) \times \operatorname{int}(Y)$.

Proof of Theorem D.4 Without loss of generality we prove the theorem for a fixed constant $p>4$. We follow the line of argument in [11,4.4.4]. Let $\varepsilon_{U h}>0$ and $C_{U h}$ be the (universal) constants in Uhlenbeck's gauge fixing theorem [32] (see also Wehrheim [34, Theorem B]). Then for each $x \in \mathbb{R} \times Y$, each sufficiently small constant $\delta>0$ with $B_{\delta}(x) \subset \mathbb{R} \times Y$, and each connection $\Xi \in \mathcal{A}(\mathbb{R} \times Y)$ with energy

$$
\int_{B_{\delta}(x)}\left|F_{\Xi}\right|^{2} \leq \varepsilon_{U h}
$$

on the geodesic ball $B_{\delta}(x)$ there is a gauge transformation $u \in \mathcal{G}(\mathbb{R} \times Y)$ such that

$$
\left\|u^{*} \Xi\right\|_{L^{4}\left(B_{\delta}(x)\right)}+\left\|u^{*} \Xi\right\|_{W^{1,2}\left(B_{\delta}(x)\right)} \leq C_{U h}\left\|F_{\Xi}\right\|_{L^{2}\left(B_{\delta}(x)\right)} .
$$

Step 1 For every $\varepsilon>0$ there is a finite set of bubbling points $S_{\varepsilon} \subset \mathbb{R} \times \operatorname{int}(Y)$ and a subsequence, still denoted by $\Xi_{v}$, such that the following holds.

(a) If $x \in(\mathbb{R} \times \operatorname{int}(Y)) \backslash S_{\varepsilon}$ then there is a $\delta>0$ with $\sup _{v} \int_{B_{\delta}(x)}\left|F_{\Xi_{v}}\right|^{2} \leq \varepsilon$.

(b) If $x \in S_{\varepsilon}$ then $\inf _{\delta>0} \liminf _{\nu \rightarrow \infty} \int_{B_{\delta}(x)}\left|F_{\Xi_{\nu}}\right|^{2} \geq \varepsilon / 2$.

Let $S_{\varepsilon}$ be the set of points $x \in \mathbb{R} \times \operatorname{int}(Y)$ that satisfy the inequality in (b). Since $X_{f}\left(\Xi_{v}\right)$ is uniformly bounded we have

$$
\inf _{\delta>0} \liminf _{\nu \rightarrow \infty} \int_{B_{\delta}(x)}\left|F_{\Xi_{\nu}}+X_{f}\left(\Xi_{\nu}\right)\right|^{2}=\inf _{\delta>0} \liminf _{\nu \rightarrow \infty} \int_{B_{\delta}(x)}\left|F_{\Xi_{\nu}}\right|^{2} \geq \frac{\varepsilon}{2}
$$


for every $x \in S_{\varepsilon}$ and hence the energy bound guarantees that $S_{\varepsilon}$ contains at most $4 E / \varepsilon$ elements. If each point in $(\mathbb{R} \times \operatorname{int}(Y)) \backslash S_{\varepsilon}$ satisfies (a) we are done. Otherwise there is a point $x \in(\mathbb{R} \times \operatorname{int}(Y)) \backslash S_{\varepsilon}$ with

$$
\inf _{\delta>0} \sup _{\nu} \int_{B_{\delta}(x)}\left|F_{\Xi_{\nu}}\right|^{2} \geq \varepsilon
$$

In this case we can choose a subsequence (still denoted by $\Xi_{v}$ ) such that

$$
\int_{B_{1 / v}(x)}\left|F_{\Xi_{v}}\right|^{2} \geq \frac{\varepsilon}{2}
$$

for all $v$. After passing to this subsequence we obtain a new strictly larger set $S_{\varepsilon}$. Continue by induction. The induction terminates when each point $x \in(\mathbb{R} \times \operatorname{int}(Y)) \backslash S_{\varepsilon}$ satisfies (a). It must terminate because in each step the set $S_{\varepsilon}$ contains at most $4 E / \varepsilon$ points.

Step 2 We denote $q:=4 p /(p+4) \in(2,4)$. If $\varepsilon>0$ is sufficiently small and $S=S_{\varepsilon}$ is as in Step 1, then there exists a subsequence, still denoted by $\Xi_{v}$, and a sequence of gauge transformations $u_{v} \in \mathcal{G}((\mathbb{R} \times Y) \backslash S)$ such that $u_{v}^{*} \Xi_{v}$ converges to $\Xi_{\infty} \in \mathcal{A}_{\text {loc }}^{1, q}((\mathbb{R} \times \operatorname{int}(Y)) \backslash S)$ in the $W^{1, q}$-norm on every compact subset of $(\mathbb{R} \times$ $\operatorname{int}(Y)) \backslash S$.

There are universal constants $C_{0} \geq 1$ and $C_{1} \geq 1$ such that

$$
\|\nabla \alpha\|_{L^{2}} \leq C_{0}\left(\left\|\mathrm{~d}^{+} \alpha\right\|_{L^{2}}+\left\|\mathrm{d}^{*} \alpha\right\|_{L^{2}}\right), \quad\|\alpha\|_{L^{4}} \leq C_{1}\|\nabla \alpha\|_{L^{2}}
$$

for $\alpha \in \Omega^{1}\left(B_{1}(0)\right)$ supported in the interior of the Euclidean unit ball. These inequalities are scale invariant, and for $\delta>0$ sufficiently small the metric in geodesic coordinates on $B_{\delta}(x)$ is $\mathcal{C}^{1}$-close up to a conformal factor to the Euclidean metric on $B_{1}(0)$. Hence the estimates (140) continue to hold with the same constants $C_{0}$ and $C_{1}$ for every compactly supported 1 -form on a geodesic ball $B_{\delta}(x) \subset \mathbb{R} \times Y$, provided that $\delta>0$ is sufficiently small.

Now fix $0<\varepsilon \leq\left(4 C_{0} C_{1} C_{U h}\right)^{-1}$ and choose a finite set $S=S_{\varepsilon} \subset \mathbb{R} \times \operatorname{int}(Y)$ and a subsequence (still denoted by $\Xi_{v}$ ) as in Step 1. Since $\varepsilon \leq \varepsilon_{U h}$ it follows from Uhlenbeck's gauge that, for every $x \in(\mathbb{R} \times \operatorname{int}(Y)) \backslash S$, there is a radius $\delta>0$ and a gauge transformation $u_{v, x} \in \mathcal{G}\left(B_{\delta}(x)\right)$ such that

$$
\left\|u_{v, x}^{*} \Xi_{v}\right\|_{W^{1,2}\left(B_{\delta}(x)\right)} \leq C_{U h^{\varepsilon}}, \quad \mathrm{d}^{*}\left(u_{v, x}^{*} \Xi_{v}\right)=0 .
$$

By a global patching argument as in [11, Lemma 4.4.5] or [34, Proposition 7.6], it suffices to construct gauge transformations, limit connections, and establish the convergence on every compact deformation retract $K \subset(\mathbb{R} \times \operatorname{int}(Y)) \backslash S$. We fix 
$K$ and find a covering by finitely many of the Uhlenbeck gauge neighbourhoods $B_{\delta_{i}}\left(x_{i}\right)$. On each of these $u_{v, x_{i}}^{*} \Xi_{v}$ satisfies (141). Now we fix a smooth cutoff function $h: B_{\delta_{i}}\left(x_{i}\right) \rightarrow[0,1]$ that vanishes near the boundary. Then

$$
\begin{aligned}
& \frac{1}{2}\left\|h \cdot u_{v, x_{i}}^{*} \Xi_{v}\right\|_{W^{2,2}} \\
& \leq C_{0}\left\|h \nabla \mathrm{d}^{+}\left(u_{v, x_{i}}^{*} \Xi_{v}\right)\right\|_{L^{2}}+C\left\|u_{v, x_{i}}^{*} \Xi_{v}\right\|_{W^{1,2}} \\
& \leq C_{0}\left\|h \nabla\left(u_{v, x_{i}}^{-1} X_{f}\left(\Xi_{v}\right)^{+} u_{v, x_{i}}-\frac{1}{2}\left[u_{v, x_{i}}^{*} \Xi_{v} \wedge u_{v, x_{i}}^{*} \Xi_{v}\right]^{+}\right)\right\|_{L^{2}}+C\left\|u_{v, x_{i}}^{*} \Xi_{v}\right\|_{W^{1,2}} \\
& \leq C_{0}\left\|u_{v, x_{i}}^{*} \Xi_{v}\right\|_{L^{4}}\left\|\nabla\left(h \cdot u_{v, x_{i}}^{*} \Xi_{v}\right)\right\|_{L^{4}}+C\left\|u_{v, x_{i}}^{*} \Xi_{v}\right\|_{L^{4}}^{2}+C\left\|u_{v, x_{i}}^{*} \Xi_{v}\right\|_{W^{1,2}} \\
&+C_{0}\left(\left\|u_{v, x_{i}}^{-1}\left(\nabla_{\Xi_{v}} X_{f}\left(\Xi_{v}\right)\right) u_{v, x_{i}}\right\|_{L^{2}}+\left\|u_{v, x_{i}}^{*} \Xi_{v}\right\|_{L^{2}}\left\|X_{f}\left(\Xi_{v}\right)\right\|_{L^{\infty}}\right) \\
& \leq C_{0} C_{1} C_{U h} \varepsilon\left\|h \cdot u_{v, x_{i}}^{*} \Xi_{v}\right\|_{W^{2,2}}+C C_{U h^{\varepsilon}}^{2}+C C_{U h^{\varepsilon}}{ }^{2} \\
&+C_{0}\left(\left\|u_{v, x_{i}}^{-1}\left(\nabla_{\Xi_{v}} X_{f}\left(\Xi_{v}\right)\right) u_{v, x_{i}}\right\|_{L^{2}}+C_{U h}\left\|X_{f}\left(\Xi_{v}\right)\right\|_{L^{\infty}}\right) .
\end{aligned}
$$

Here all norms are in $B_{\delta_{i}}\left(x_{i}\right)$ and $C$ denotes a constant that only depends on $h$ and the radius $\delta_{i}$. In the first step we have used (140) with $\alpha=\partial_{i}\left(h \cdot u_{v, x_{i}}^{*} \Xi_{v}\right), i=1, \ldots, 4$, and (141). In the last step we have used (141) and the inequality

$$
\left\|\nabla\left(h \cdot u_{v, x_{i}}^{*} \Xi_{v}\right)\right\|_{L^{4}} \leq C_{1}\left\|h \cdot u_{v, x_{i}}^{*} \Xi_{v}\right\|_{W^{2,2}}
$$

of (140). Since $C_{0} C_{1} C_{U h} \varepsilon \leq 1 / 4$ and

$$
\left\|\nabla_{\Xi_{\nu}} X_{f}\left(\Xi_{v}\right)\right\|_{L^{2}\left(B_{\delta_{i}}\left(x_{i}\right)\right)} \leq C\left(1+\left\|F_{\Xi_{\nu}}\right\|_{L^{2}(I \times Y)}\right)
$$

for an interval $I \subset \mathbb{R}$ with $B_{\delta_{i}}\left(x_{i}\right) \subset I \times Y$ we obtain a $W^{2,2}$-bound on $u_{v, x_{i}}^{*} \Xi_{v}$ over a slightly smaller ball in $B_{\delta_{i}}\left(x_{i}\right)$ where $h \equiv 1$.

By Uhlenbeck's patching procedure [32] (see also Wehrheim [34, Chapter 7]) the gauge transformations $u_{v, x_{i}}$ can then be interpolated to find $u_{v} \in \mathcal{G}(K)$ such that $u_{v}^{*} \Xi_{v}$ is bounded in $W^{2,2}(K)$. The compact Sobolev embedding $W^{2,2}(K) \hookrightarrow W^{1, q}(K)$ for $q<4$ then provides a $W^{1, q}$-convergent subsequence $u_{v}^{*} \Xi_{v} \rightarrow \Xi_{\infty} \in \mathcal{A}^{1, q}(K)$.

Step 3 We prove the theorem with $\hbar=\varepsilon / 4$ where $\varepsilon$ is as in Step 2. In particular, we remove the singularities to find $\widetilde{\Xi}_{\infty} \in \mathcal{A}(\mathbb{R} \times \operatorname{int}(Y))$, a subsequence, and gauge transformations $\tilde{u}_{v} \in \mathcal{G}((\mathbb{R} \times \operatorname{int}(Y)) \backslash S)$ such that $\tilde{u}_{v}^{*} \Xi_{v} \rightarrow \widetilde{\Xi}_{\infty}$ in the $W^{1, p}$-norm on every compact subset of $(\mathbb{R} \times \operatorname{int}(Y)) \backslash S$.

Step 2 gives $u_{v}^{*} \Xi_{v} \rightarrow \Xi_{\infty} \in \mathcal{A}_{\text {loc }}^{1, q}((\mathbb{R} \times \operatorname{int}(Y)) \backslash S)$ with $q>2$. This implies $L^{2}-$ convergence of the curvature on every compact subset of $(\mathbb{R} \times \operatorname{int}(Y)) \backslash S$, and hence 
with the exhausting sequence $K_{\delta}:=\left(\left[-\delta^{-1}, \delta^{-1}\right] \times Y\right) \backslash B_{\delta}(S \cup \mathbb{R} \times \partial Y)$

$$
\int_{\mathbb{R} \times Y}\left|F_{\Xi_{\infty}}\right|^{2}=\lim _{\delta \rightarrow 0} \int_{K_{\delta}}\left|F_{\Xi_{\infty}}\right|^{2}=\lim _{\delta \rightarrow 0} \lim _{\nu \rightarrow \infty} \int_{K_{\delta}}\left|F_{\Xi_{\nu}}\right|^{2} \leq E .
$$

Next we consider small annuli around the singularities and denote their union, for $k \in \mathbb{N}$ sufficiently large, by

$$
A_{k}:=B_{2^{1-k}}(S) \backslash B_{2^{-k}}(S)
$$

Then $\int_{A_{k}}\left|F_{\Xi_{\infty}}\right|^{2} \rightarrow 0$ as $k \rightarrow \infty$ since the above limit exists. For sufficiently large $k$ we can now patch Uhlenbeck gauges to obtain a gauge transformation $u_{k} \in \mathcal{G}\left(A_{k}\right)$ such that $\left\|u_{k}^{*} \Xi_{\infty}\right\|_{L^{4}\left(A_{k}\right)} \leq C\left\|F_{\Xi_{\infty}}\right\|_{L^{2}\left(A_{k}\right)} \rightarrow 0$. The patching procedure does not introduce $k$-dependent constants or a flat connection since the inequality is scale invariant and each annulus can be covered by two balls whose intersection is connected and simply connected (see [11, 4.4.10]).

We extend $u_{k}$ to $(\mathbb{R} \times Y) \backslash S$ and denote

$$
\Xi_{k}^{\prime}:=\left(u_{v_{k}} u_{k}\right)^{*} \Xi_{v_{k}}
$$

Here we pick a subsequence $v_{k} \rightarrow \infty$ such that

$$
\left\|F_{u_{v_{k}}^{*} \Xi_{v_{k}}}\right\|_{L^{2}\left(A_{k_{0}}\right)}^{2} \leq 2\left\|F_{\Xi_{\infty}}\right\|_{L^{2}\left(A_{k_{0}}\right)}^{2}
$$

for all $k \geq k_{0}$ sufficiently large, and

$$
\lim _{k \rightarrow \infty} \sup _{\ell \geq k}\left\|u_{v_{\ell}}^{*} \Xi_{v_{\ell}}-\Xi_{\infty}\right\|_{L^{4}\left(A_{k}\right)}=0
$$

In particular, we have $\left\|\Xi_{k}^{\prime}\right\|_{L^{4}\left(A_{k}\right)} \rightarrow 0$ as $k \rightarrow \infty$. Now consider the sequence of extended connections

$$
\widetilde{\Xi}_{k}:=h_{k} \cdot \Xi_{k}^{\prime} \in \mathcal{A}(\mathbb{R} \times Y),
$$

where $h_{k}: \mathbb{R} \times Y \rightarrow[0,1]$ is a cutoff function that vanishes on $B_{2^{-k}}(S)$, varies smoothly on $A_{k}$ with $\left|\mathrm{d} h_{k}\right| \leq 2^{k+1}$, and equals to 1 on the complement of $B_{2^{1-k}}(S)$. The curvature of the extended connections is

$$
F_{\widetilde{\Xi}_{k}}=h_{k} \cdot F_{\Xi_{k}^{\prime}}+\frac{1}{2}\left(h_{k}^{2}-h_{k}\right)\left[\Xi_{k}^{\prime} \wedge \Xi_{k}^{\prime}\right]+\mathrm{d} h_{k} \wedge \Xi_{k}^{\prime} .
$$


So for $\delta=2^{-\ell}$, and $k \geq \ell+1$ we have

$$
\begin{aligned}
& \int_{B_{\delta}(S)}\left|F_{\Xi_{k}}\right|^{2} \\
& \leq 2 \int_{B_{\delta}(S) \backslash B_{21-k}(S)}\left|F_{\Xi_{\infty}}\right|^{2}+\int_{A_{k}}\left(h_{k}^{2}\left|F_{\Xi_{k}^{\prime}}\right|^{2}+\left|h_{k}^{2}-h_{k}\right|^{2}\left|\Xi_{k}^{\prime}\right|^{4}+\left|\mathrm{d} h_{k}\right|^{2} \cdot\left|\Xi_{k}^{\prime}\right|^{2}\right) \\
& \leq 2 \int_{B_{\delta}(S) \backslash B_{2-k}(S)}\left|F_{\Xi_{\infty}}\right|^{2}+\left\|\Xi_{k}^{\prime}\right\|_{L^{4}\left(A_{k}\right)}^{4}+2^{2 k+2} \operatorname{Vol}\left(A_{k}\right)^{\frac{1}{2}}\left\|\Xi_{k}^{\prime}\right\|_{L^{4}\left(A_{k}\right)}^{2} .
\end{aligned}
$$

The right hand side converges to

$$
2 \int_{B_{\delta}(S)}\left|F_{\Xi_{\infty}}\right|^{2}
$$

as $k \rightarrow \infty$, so for sufficiently small $\delta=2^{-\ell}$ we have locally small energy

$$
\sup _{k} \int_{B_{\delta}(x)}\left|F_{\widetilde{\Xi}_{k}}\right|^{2} \leq \varepsilon
$$

at every $x \in \mathbb{R} \times \operatorname{int}(Y)$ for the subsequence $\left(\widetilde{\Xi}_{k}\right)_{k \geq \ell}$. (For $x \notin S$ this is true by Step 1.)

Now we can find an Uhlenbeck gauge $v_{k} \in \mathcal{G}\left(B_{\delta}(S)\right)$ such that

$$
\mathrm{d}^{*}\left(v_{k}^{*} \widetilde{\Xi}_{k}\right)=0, \quad\left\|v_{k}^{*} \widetilde{\Xi}_{k}\right\|_{W^{1,2}\left(B_{\delta}(S)\right)} \leq C_{U h^{\varepsilon}} \varepsilon
$$

The $W^{1,2}$-bound allows us to choose a $W^{1,2}$-weakly convergent subsequence

$$
v_{k}^{*} \widetilde{\Xi}_{k} \rightarrow \widetilde{\Xi}_{\infty} \in \mathcal{A}^{1,2}\left(B_{\delta}(S)\right) .
$$

On the other hand, for every closed ball $D \subset B_{\delta}(S) \backslash S$ and every sufficiently large $k$ (such that $\left.h_{k}\right|_{D} \equiv 1$ ) the same estimate as in Step 2 provides $W^{2,2}$-bounds on $\left.v_{k}^{*} \widetilde{\Xi}_{k}\right|_{D}$ and thus $W^{1, q}$-convergence $v_{k}^{*} \widetilde{\Xi}_{k} \rightarrow \widetilde{\Xi}_{\infty} \in \mathcal{A}_{\text {loc }}^{1, q}\left(B_{\delta}(S) \backslash S\right)$ on every compact subset.

We can extend the gauge transformations $v_{k} \in \mathcal{G}\left(B_{\delta}(S)\right)$ by Uhlenbeck's patching procedure to a compact deformation retract $S \subset K \subset \mathbb{R} \times \operatorname{int}(Y)$ (which is covered by $B_{\delta}(S)$ and finitely many balls in $(\mathbb{R} \times \operatorname{int}(Y)) \backslash S$ on which we also have an Uhlenbeck gauge and hence $W^{2,2}$-bounds), and to $\mathbb{R} \times \operatorname{int}(Y)$ by the general extension procedure [34, Proposition 7.6]. This provides a subsequence and gauge transformations $v_{k} \in \mathcal{G}(\mathbb{R} \times \operatorname{int}(Y))$ such that the $v_{k}^{*} \widetilde{\Xi}_{k}$ converge in the $W^{1, q}$-norm on every compact subset of $(\mathbb{R} \times \operatorname{int}(Y)) \backslash S$ to a limit connection $\widetilde{\Xi}_{\infty} \in \mathcal{A}_{\text {loc }}^{1, q}((\mathbb{R} \times \operatorname{int}(Y)) \backslash S)$. In particular, this means that

$$
\tilde{u}_{v_{k}}^{*} \Xi_{v_{k}} \rightarrow \widetilde{\Xi}_{\infty}, \quad \tilde{u}_{v_{k}}:=u_{v_{k}} u_{k} v_{k} \in \mathcal{G}((\mathbb{R} \times \operatorname{int}(Y)) \backslash S),
$$


because $\widetilde{\Xi}_{k}=\left(u_{v_{k}} u_{k}\right)^{*} \Xi_{v_{k}}$ on compact subsets of $(\mathbb{R} \times \operatorname{int}(Y)) \backslash S$. Moreover, the limit connection extends to $S$ such that $v_{k}^{*} \widetilde{\Xi}_{k} \rightarrow \widetilde{\Xi}_{\infty} \in \mathcal{A}^{1,2}\left(B_{\delta}(S)\right)$ converges $W^{1,2}$-weakly and $L^{4}$-weakly.

Since $\widetilde{\Xi}_{\infty}$ is of class $W^{1,2}$, the perturbation $X_{f}\left(\widetilde{\Xi}_{\infty}\right) \in L^{\infty}(\mathbb{R} \times Y)$ is well defined, and we claim that

$$
X_{f}\left(v_{k}^{*} \widetilde{\Xi}_{k}\right) \rightarrow X_{f}\left(\widetilde{\Xi}_{\infty}\right), \quad \tilde{u}_{v_{k}}^{-1} X_{f}\left(\Xi_{v_{k}}\right) \tilde{u}_{v_{k}} \rightarrow X_{f}\left(\widetilde{\Xi}_{\infty}\right)
$$

in the $L^{p}$-norm on every compact subset of $\mathbb{R} \times Y$. If $S$ does not intersect the support $\operatorname{supp} X_{f}:=\bigcup_{i=1}^{N} \mathbb{R} \times \operatorname{im} \gamma_{i}$ of the perturbation then $\left.\widetilde{\Xi}_{\infty}\right|_{\text {supp } X_{f}}$ is the $W_{\text {loc }}^{1, q}-$ limit of $\left.v_{k}^{*} \widetilde{\Xi}_{k}\right|_{\operatorname{supp} X_{f}}=\left.v_{k}^{*} \Xi_{k}^{\prime}\right|_{\operatorname{supp} X_{f}}=\left.\tilde{u}_{v_{k}}^{*} \Xi_{v_{k}}\right|_{\operatorname{supp} X_{f}}$ and the claim follows directly from Remark D.2 and the Sobolev embedding $W^{1, q} \hookrightarrow L^{p}$ on compact subsets of $\mathbb{R} \times Y$. If $S$ does intersect the set $\operatorname{supp} X_{f}$ at some points $\left(s_{j}, \gamma_{i_{j}}\left(\theta_{j}, z_{j}\right)\right)_{j=1, \ldots, n} \subset S$, then we have

$$
X_{f}\left(v_{k}^{*} \widetilde{\Xi}_{k}\right)=v_{k}^{-1} X_{f}\left(h_{k} \Xi_{k}^{\prime}\right) v_{k}=v_{k}^{-1} X_{f}\left(\Xi_{k}^{\prime}\right) v_{k}=\tilde{u}_{v_{k}}^{-1} X_{f}\left(\Xi_{v_{k}}\right) \tilde{u}_{v_{k}}
$$

only on the complement of a solid cylinder neighbourhood (denoted by $Z_{k}$ ) of the loops $\left(s_{j}, \gamma_{i_{j}}\left(S^{1}, z_{j}\right)\right) \subset \mathbb{R} \times Y$. More precisely, $Z_{k} \subset \mathbb{R} \times \operatorname{int}(Y)$ is given by the union of all loops $\left(s, \gamma_{i}\left(S^{1}, z\right)\right)$ that intersect the support of $1-h_{k}$. It thus is a union of solid cylinders whose width is of order $2^{1-k}$. If we fix the cylinder neighbourhood $Z_{k_{0}}$, then the previous argument still applies for $k \geq k_{0}$ to give $L^{p}$-convergence on the complement of $Z_{k_{0}}$. The remaining $Z_{k_{0}}$ has volume of order $2^{3-3 k_{0}}$, and the perturbations $X_{f}\left(\widetilde{\Xi}_{\infty}\right), X_{f}\left(v_{k}^{*} \widetilde{\Xi}_{k}\right)$, and $X_{f}\left(\Xi_{v_{k}}\right)$ are all uniformly bounded by Proposition D.1 (iii) (with $k=0)$. So we see that $\left\|X_{f}\left(v_{k}^{*} \widetilde{\Xi}_{k}\right)-X_{f}\left(\widetilde{\Xi}_{\infty}\right)\right\|_{L^{p}\left(Z_{k_{0}}\right)}$ and $\left\|\tilde{u}_{v_{k}}^{-1} X_{f}\left(\Xi_{v_{k}}\right) \tilde{u}_{v_{k}}-X_{f}\left(\widetilde{\Xi}_{\infty}\right)\right\|_{L^{p}\left(Z_{k_{0}}\right)}$ also converge to zero as we let $k \geq k_{0} \rightarrow \infty$. This proves (143).

A first consequence is that the limit connection satisfies

$$
\left(F \widetilde{\Xi}_{\infty}+X_{f}\left(\widetilde{\Xi}_{\infty}\right)\right)^{+}=0
$$

because this is the local weak $L^{2}$-limit of $\left(F_{v_{k}^{*}} \widetilde{\Xi}_{k}+X_{f}\left(v_{k}^{*} \widetilde{\Xi}_{k}\right)\right)^{+}$and

$$
\begin{aligned}
& \left\|\left(F_{v_{k}^{*} \widetilde{\Xi}_{k}}+X_{f}\left(v_{k}^{*} \widetilde{\Xi}_{k}\right)\right)^{+}\right\|_{L^{2}(\mathbb{R} \times Y)} \\
& =\left\|\left(F_{h_{k}} \Xi_{k}^{\prime}+X_{f}\left(h_{k} \Xi_{k}^{\prime}\right)\right)^{+}-\left(u_{v_{k}} u_{k}\right)^{-1}\left(F_{\Xi_{v_{k}}}+X_{f}\left(\Xi_{v_{k}}\right)\right)^{+}\left(u_{v_{k}} u_{k}\right)\right\|_{L^{2}(\mathbb{R} \times Y)} \\
& \leq\left\|F_{h_{k} \Xi_{k}^{\prime}}-F_{\Xi_{k}^{\prime}}\right\|_{L^{2}\left(A_{k}\right)}+\left\|X_{f}\left(h_{k} \Xi_{k}^{\prime}\right)-\left(u_{v_{k}} u_{k}\right)^{-1} X_{f}\left(\Xi_{v_{k}}\right)\left(u_{v_{k}} u_{k}\right)\right\|_{L^{2}\left(Z_{k}\right)},
\end{aligned}
$$

which converges to zero by similar estimates as before. Another consequence is the energy identity: We have

$$
F_{v_{k}^{*}} \widetilde{\Xi}_{k}+X_{f}\left(v_{k}^{*} \widetilde{\Xi}_{k}\right) \rightarrow F \widetilde{\Xi}_{\infty}+X_{f}\left(\widetilde{\Xi}_{\infty}\right)
$$


in the $L^{2}$-norm on every compact subset of $(\mathbb{R} \times \operatorname{int}(Y)) \backslash S$. So, exhausting $\mathbb{R} \times Y$ with

$$
K_{\delta}:=\left(\left[-\delta^{-1}, \delta^{-1}\right] \times Y\right) \backslash B_{\delta}(S \cup \mathbb{R} \times \partial Y),
$$

we have

$$
\begin{aligned}
& E_{f}\left(\widetilde{\Xi}_{\infty}\right) \\
& =\lim _{\delta \rightarrow 0} \frac{1}{2} \int_{K_{\delta}}\left|F \widetilde{\Xi}_{\infty}+X_{f}\left(\widetilde{\Xi}_{\infty}\right)\right|^{2}=\lim _{\delta \rightarrow 0} \lim _{k \rightarrow \infty} \frac{1}{2} \int_{K_{\delta}}\left|F \widetilde{\Xi}_{k}+X_{f}\left(\widetilde{\Xi}_{k}\right)\right|^{2} \\
& \leq \lim _{\delta \rightarrow 0} \lim _{k \rightarrow \infty} \frac{1}{2}\left(\int_{K_{\delta}}\left|F_{\Xi_{v_{k}}}+X_{f}\left(\Xi_{v_{k}}\right)\right|^{2}+\int_{K_{\delta}}\left|F_{h_{k} \Xi_{k}^{\prime}}-F_{\Xi_{k}^{\prime}}\right|^{2}\right. \\
& \left.+\int_{K_{\delta}}\left|X_{f}\left(h_{k} \Xi_{k}^{\prime}\right)-\left(u_{v_{k}} u_{k}\right)^{-1} X_{f}\left(\Xi_{v_{k}}\right)\left(u_{v_{k}} u_{k}\right)\right|^{2}\right) \\
& =\lim _{\delta \rightarrow 0} \lim _{k \rightarrow \infty} \frac{1}{2}\left(\int_{\left[-\delta^{-1}, \delta^{-1}\right] \times Y}\left|F_{\Xi_{v_{k}}}+X_{f}\left(\Xi_{v_{k}}\right)\right|^{2}-\int_{B_{\delta}(S)}\left|F_{\Xi_{v_{k}}}+X_{f}\left(\Xi_{v_{k}}\right)\right|^{2}\right) \\
& \leq \limsup _{\nu \rightarrow \infty} E_{f}\left(\Xi_{v}\right)-N \hbar \text {. }
\end{aligned}
$$

Here $\hbar:=\varepsilon / 4$ with $\varepsilon>0$ as in Step 2 .

It follows from (142) and (144) that

$$
\mathrm{d}^{*} \widetilde{\Xi}_{\infty}=0, \quad\left\|\widetilde{\Xi}_{\infty}\right\|_{L^{4}\left(B_{\delta}(S)\right)} \leq C_{U h^{\varepsilon}}, \quad F_{\widetilde{\Xi}_{\infty}}^{+} \in L^{\infty}(\mathbb{R} \times Y) .
$$

This implies $\widetilde{\Xi}_{\infty} \in \mathcal{A}^{1,3}\left(B_{\delta / 2}(S)\right)$ by a standard argument as in [11, Proposition 4.4.13], using the estimate

$$
\|\mathbb{A}\|_{W^{1,3}} \leq C\left(\left\|\mathrm{~d}^{*} \mathbb{A}\right\|_{L^{3}}+\left\|F_{\mathbb{A}}^{+}\right\|_{L^{3}}+\|\mathbb{A}\|_{L^{4}}\|\mathbb{A}\|_{W^{1,3}}\right)
$$

for compactly supported $\mathbb{A} \in \mathcal{A}\left(B_{\delta}(S)\right)$. Hence we have $\widetilde{\Xi}_{\infty} \in \mathcal{A}_{\text {loc }}^{1,3}(\mathbb{R} \times \operatorname{int}(Y))$. Now the standard regularity theory for anti-self-dual connections (eg [34, Chapter 9]) together with Remark D.2, for control of the perturbation, provides another gauge transformation that makes $\widetilde{\Xi}_{\infty}$ smooth and does not affect the convergence.

It remains to strengthen the convergence

$$
\tilde{u}_{v_{k}}^{*} \Xi_{v^{k}} \rightarrow \widetilde{\Xi}_{\infty}
$$

on $(\mathbb{R} \times \operatorname{int}(Y)) \backslash S$ to the $W_{\text {loc }}^{1, p}$-topology. Again, it suffices to construct the required subsequence and gauge transformations on a compact deformation retract $K \subset(\mathbb{R} \times$ $\operatorname{int}(Y)) \backslash S$. We pick a compact submanifold

$$
M \subset(\mathbb{R} \times \operatorname{int}(Y)) \backslash S
$$


such that $K \subset \operatorname{int}(M)$ and apply the local slice theorem (eg [34, Theorem 8.1]) to find gauge transformations $u_{v_{k}} \in \mathcal{G}(M)$ such that

$$
\mathrm{d}_{\Xi_{\infty}}^{*}\left(u_{v_{k}}^{*} \Xi_{v_{k}}-\widetilde{\Xi}_{\infty}\right)=0, \quad \lim _{k \rightarrow \infty}\left\|u_{v_{k}}^{*} \Xi_{v_{k}}-\widetilde{\Xi}_{\infty}\right\|_{W^{1, q}(M)}=0 .
$$

Since $\left.\widetilde{u}_{v_{k}}^{*} \Xi_{v_{k}}\right|_{M}$ has the same $W^{1, q}$-limit, the gauge transformations $\widetilde{u}_{v_{k}}^{-1} u_{v_{k}} \in \mathcal{G}(M)$ converge, for a further subsequence, in the weak $W^{2, q}(M)$-topology to an element $u_{\infty}$ of the isotropy subgroup of $\widetilde{\Xi}_{\infty}$. We can make sure that this limit is in fact 1 , by modifying $u_{v_{k}}$ to $u_{v_{k}} u_{\infty}^{-1}$ in the local slice gauge. With this we have

$$
\lim _{k \rightarrow \infty}\left\|u_{v_{k}}^{*} \Xi_{v_{k}}-\widetilde{\Xi}_{\infty}\right\|_{L^{p}(M)}=0, \quad \lim _{k \rightarrow \infty}\left\|\mathrm{d}^{*}\left(u_{v_{k}}^{*} \Xi_{v_{k}}-\widetilde{\Xi}_{\infty}\right)\right\|_{L^{p}(M)}=0,
$$

so we can use the elliptic estimate for $\mathrm{d}^{+} \oplus \mathrm{d}^{*}$ on $M$. For that purpose fix a cutoff function $h: M \rightarrow[0,1]$ with $\left.h\right|_{K} \equiv 1$ and $h \equiv 0$ near $\partial M$. Then

$$
\begin{aligned}
\left\|\mathrm{d}^{+}\left(h\left(u_{v_{k}}^{*} \Xi_{v_{k}}-\widetilde{\Xi}_{\infty}\right)\right)\right\|_{p} & \\
\leq C_{h}\left\|u_{v_{k}}^{*} \Xi_{v_{k}}-\widetilde{\Xi}_{\infty}\right\|_{p} & +\left\|X_{f}\left(\widetilde{\Xi}_{\infty}\right)-u_{v_{k}}^{-1} X_{f}\left(\Xi_{v_{k}}\right) u_{v_{k}}\right\|_{p} \\
& +\left\|h\left[\widetilde{\Xi}_{\infty} \wedge \widetilde{\Xi}_{\infty}\right]^{+}-h\left[u_{v_{k}}^{*} \Xi_{v_{k}} \wedge u_{v_{k}}^{*} \Xi_{v_{k}}\right]^{+}\right\|_{p} .
\end{aligned}
$$

Here the constant $C_{h}:=\|\nabla h\|_{\infty}$ is finite, so the first term converges to zero as $k \rightarrow \infty$. The second term also converges to zero due to (143) and the $\mathcal{C}^{0}$-convergence $\tilde{u}_{v_{k}}^{-1} u_{v_{k}} \rightarrow 11$. Finally, the third term can be bounded by the constant

$$
\left(2\left\|\widetilde{\Xi}_{\infty}\right\|_{L^{\infty}}+C_{S}\left\|h\left(u_{v_{k}}^{*} \Xi_{v_{k}}-\widetilde{\Xi}_{\infty}\right)\right\|_{W^{1, p}}\right)\left\|u_{v_{k}}^{*} \Xi_{v_{k}}-\widetilde{\Xi}_{\infty}\right\|_{L^{p}}
$$

with a constant $C_{S}$ from the Sobolev embedding $W^{1, p}(M) \hookrightarrow \mathcal{C}^{0}(M)$. Now apply the elliptic estimate for $\mathrm{d}^{+} \oplus \mathrm{d}^{*}$ to the compactly supported 1 -form $\eta_{k}:=h\left(u_{v_{k}}^{*} \Xi_{v_{k}}-\widetilde{\Xi}_{\infty}\right)$ to obtain

$\left\|\eta_{k}\right\|_{W^{1, p}} \leq C\left(1+\left\|\eta_{k}\right\|_{W^{1, p}}\right)\left\|u_{v_{k}}^{*} \Xi_{v_{k}}-\widetilde{\Xi}_{\infty}\right\|_{p}+C\left\|X_{f}\left(\widetilde{\Xi}_{\infty}\right)-u_{v_{k}}^{-1} X_{f}\left(\Xi_{v_{k}}\right) u_{v_{k}}\right\|_{p}$

with a finite constant $C$. Since $\left\|u_{v_{k}}^{*} \Xi_{v_{k}}-\widetilde{\Xi}_{\infty}\right\|_{L^{p}(M)} \rightarrow 0$ this can be rearranged to prove that

$$
\left\|u_{v_{k}}^{*} \Xi_{v_{k}}-\widetilde{\Xi}_{\infty}\right\|_{W^{1, p}(K)} \leq\left\|\eta_{k}\right\|_{W^{1, p}(M)} \rightarrow 0 .
$$

This finishes the proof of Step 3 and the theorem. 


\section{Appendix E The Lagrangian and its tangent bundle}

For any compact manifold $X$, any integer $k \geq 0$, and any $p>1$ we denote the space of $W^{k, p}$-connections by

$$
\mathcal{A}^{k, p}(X):=W^{k, p}\left(X, \mathrm{~T}^{*} X \otimes \mathfrak{g}\right) .
$$

If $(k+1) p>\operatorname{dim} X$ then the gauge group

$$
\mathcal{G}^{k+1, p}(X):=W^{k+1, p}(X, \mathrm{G})
$$

acts smoothly on $\mathcal{A}^{k, p}(X)$. For $p=\infty$ we denote by $\mathcal{A}^{k, \infty}(X)$ the space of $\mathcal{C}^{k}$ connections; similarly for $\mathcal{G}^{k, \infty}(X)$.

Let $Y$ be a compact oriented Riemannian 3-manifold with boundary $\partial Y=\Sigma$ and $\mathcal{L} \subset \mathcal{A}(\Sigma)$ be a gauge invariant Lagrangian submanifold (in the sense of (L1) of the introduction) such that $\mathcal{L} / \mathcal{G}_{z}(\Sigma)$ is compact. For $(k+1) p>2$ the $W^{k, p}$-closure of $\mathcal{L}$ is a Banach submanifold of $\mathcal{A}^{k, p}(\Sigma)$, which we denote by $\mathcal{L}^{k, p}$. (This follows from the Sobolev embedding $W^{k, p}(\Sigma) \hookrightarrow L^{q}(\Sigma)$ with $q>2$ and the fact that the $L^{q}-$ Banach submanifold coordinates in [33, Lemma 4.3] restrict to $W^{k, p}$-coordinates.) Again, we denote by $\mathcal{L}^{k, \infty}$ the $\mathcal{C}^{k}$-completion. Denote

$$
\mathcal{A}^{k, p}(Y, \mathcal{L}):=\left\{A \in \mathcal{A}^{k, p}(Y)|A|_{\partial Y} \in \mathcal{L}^{0, q}\right\} .
$$

This is a Banach submanifold of $\mathcal{A}^{k, p}(Y)$ for $(k+1) p>3$ since the restriction map $\mathcal{A}^{k, p}(Y) \hookrightarrow \mathcal{A}^{0, q}(\Sigma)$ with $q>2$ is smooth and transverse to $\mathcal{L}$. Theorem E.4 will provide a gauge equivariant exponential map for $\mathcal{A}^{1, p}(Y, \mathcal{L})$, from which we construct an exponential map for $\mathcal{A}^{1, p}\left(\mathbb{R} \times Y, \mathcal{L} ; B_{-}, B_{+}\right)$in Corollary E.5.

Moreover, consider the vector bundle $\mathcal{E} \rightarrow \mathcal{A}(Y, \mathcal{L})$ with fibre

$$
\mathcal{E}_{A}:=\Omega_{A}^{1}(Y, \mathfrak{g})=\left\{\alpha \in \Omega^{1}(Y, \mathfrak{g})|* \alpha|_{\partial Y}=0,\left.\alpha\right|_{\partial Y} \in \mathrm{T}_{A} \mathcal{L}\right\} .
$$

In Theorem E. 2 below we construct local trivializations of $\mathcal{E}$. In a preliminary step we construct local trivializations of the tangent bundle of $\mathcal{L}$. Note that these trivializations extend to the fibrewise $L^{2}$-closure of the tangent bundle although it is not known whether the $L^{2}$-closure of $\mathcal{L}$ is smooth.

Theorem E.1 For every $A_{0} \in \mathcal{L}$ there exists a neighbourhood $\mathcal{U} \subset \mathcal{L}$ of $A_{0}$ (open in the $\mathcal{C}^{0}$-topology) and a family of bijective linear operators

$$
P_{A}: \Omega^{1}(\Sigma, \mathfrak{g}) \rightarrow \Omega^{1}(\Sigma, \mathfrak{g}),
$$

parametrized by $A \in \mathcal{U}$, such that the following holds. 
(i) $P_{A_{0}}=11$.

(ii) For every $A \in \mathcal{U}$ and every $\alpha \in \Omega^{1}(\Sigma, \mathfrak{g})$ we have

$$
P_{A} \alpha \in \mathrm{T}_{A} \mathcal{L} \quad \Longleftrightarrow \quad \alpha \in \mathrm{T}_{A_{0}} \mathcal{L} .
$$

(iii) For every integer $k \geq 0$ and every $p>1$ the operator $P_{A}$ extends to a Banach space isomorphism from $W^{k, p}\left(\Sigma, \mathrm{T}^{*} \Sigma \otimes \mathfrak{g}\right)$ to itself; this extended operator depends smoothly on $A \in \mathcal{L}^{k, \infty}$ with respect to the operator norm on $P_{A}$.

(iv) For every integer $k \geq 0$, every $p>1$, every $\lambda \in[0,1]$, and every $A \in \mathcal{U}^{k, \infty}$ the operator $\lambda 11+(1-\lambda) P_{A}$ extends to a Banach space isomorphism from $W^{k, p}\left(\Sigma, \mathrm{T}^{*} \Sigma \otimes \mathfrak{g}\right)$ to itself. Here $\mathcal{U}^{k, \infty}$ denotes the interior of the closure of $\mathcal{U}$ in $\mathcal{L}^{k, \infty}$.

Proof Choose a 3-dimensional subspace $E \subset \Omega^{0}(\Sigma, \mathfrak{g})$ such that the restriction of $\mathrm{d}_{A_{0}}: \Omega^{0}(\Sigma, \mathfrak{g}) \rightarrow \Omega^{1}(\Sigma, \mathfrak{g})$ to $E^{\perp}$ (the $L^{2}$-orthogonal complement of $E$ ) is injective. Then there is a constant $C$ such that $\|\xi\|_{W^{1,2}} \leq C\left\|\mathrm{~d}_{A_{0}} \xi\right\|_{L^{2}}$ for all $\xi \in E^{\perp}$. This estimate continues to hold for each $A \in \mathcal{L}$ that is sufficiently close to $A_{0}$ in the $\mathcal{C}^{0}$-norm. Hence there is a $\mathcal{C}^{0}$-open neighbourhood $\mathcal{U} \subset \mathcal{L}$ of $A_{0}$ such that $\mathrm{d}_{A}: E^{\perp} \rightarrow \Omega^{1}(\Sigma, \mathfrak{g})$ is injective for every $A \in \mathcal{U}$. Define

$$
H_{A, E}^{1}:=\left\{\alpha \in \Omega^{1}(\Sigma, \mathfrak{g}) \mid * \mathrm{~d}_{A} \alpha \in E, \mathrm{~d}_{A}^{*} \alpha \in E\right\} .
$$

Then, for every $A \in \mathcal{U}$, there is a generalized Hodge decomposition

$$
\Omega^{1}(\Sigma, \mathfrak{g})=H_{A, E}^{1} \oplus \mathrm{d}_{A}\left(E^{\perp}\right) \oplus * \mathrm{~d}_{A}\left(E^{\perp}\right) .
$$

The three summands in (145) are orthogonal to each other and the generalized Hodge decomposition extends to each Sobolev completion $\mathcal{A}^{k, p}(\Sigma)$ in the usual fashion. This uses the fact that the operator

$$
\Delta_{A, E}:=\mathrm{d}_{A}^{*} \mathrm{~d}_{A}: \Omega^{0}(\Sigma, \mathfrak{g}) \supset E^{\perp} \rightarrow \Omega^{0}(\Sigma, \mathfrak{g}) / E
$$

extends to an isomorphism from $W^{k+2, p}$ to $W^{k, p}$ (with $p>1$ ) for every $A \in \mathcal{U}$. (The operators $\Delta_{A, E}$ are all injective and compact perturbations of the isomorphism $\Delta_{A_{0}, E}$.) The standard Hodge decomposition corresponds to the case $E=\operatorname{ker}_{A}$. The reason for our construction with $E$ independent of $A$ is the need for a Hodge decomposition which depends smoothly on $A$.

The Lagrangian submanifold $\mathcal{L}$ gives rise to another $L^{2}$-orthogonal decomposition, $\Omega^{1}(\Sigma, \mathfrak{g})=\mathrm{T}_{A} \mathcal{L} \oplus * \mathrm{~T}_{A} \mathcal{L}$, see [33, Lemma 4.2]. Since $\mathrm{d}_{A}\left(E^{\perp}\right) \subset \mathrm{T}_{A} \mathcal{L}$ and $* \mathrm{~d}_{A}\left(E^{\perp}\right)$ is perpendicular to $\mathrm{T}_{A} \mathcal{L}$ it follows from (145) that we have $\mathrm{T}_{A} \mathcal{L}=\Lambda_{A} \oplus \mathrm{d}_{A}\left(E^{\perp}\right)$, where

$$
\Lambda_{A}:=H_{A, E}^{1} \cap \mathrm{T}_{A} \mathcal{L}
$$


is a Lagrangian subspace of $H_{A, E}^{1}$. Hence there is a refined Hodge decomposition

$$
\Omega^{1}(\Sigma, \mathfrak{g})=\Lambda_{A} \oplus * \Lambda_{A} \oplus \mathrm{d}_{A}\left(E^{\perp}\right) \oplus * \mathrm{~d}_{A}\left(E^{\perp}\right) .
$$

For $A \in \mathcal{U}$ we define a bijective linear operator $P_{A}: \Omega^{1}(\Sigma, \mathfrak{g}) \rightarrow \Omega^{1}(\Sigma, \mathfrak{g})$ by

$$
P_{A}\left(\alpha_{0}+* \beta_{0}+\mathrm{d}_{A_{0}} \xi+* \mathrm{~d}_{A_{0}} \eta\right):=\Pi_{A} \alpha_{0}+* \Pi_{A} \beta_{0}+\mathrm{d}_{A} \xi+* \mathrm{~d}_{A} \eta
$$

for $\alpha_{0}, \beta_{0} \in \Lambda_{A_{0}}$ and $\xi, \eta \in E^{\perp} \subset \Omega^{0}(\Sigma, \mathfrak{g})$, where

$$
\Pi_{A}: \Omega^{1}(\Sigma, \mathfrak{g}) \rightarrow \Lambda_{A}
$$

denotes the $L^{2}$-orthogonal projection. (Shrink $\mathcal{U}$, if necessary, so that the restriction of $\Pi_{A}$ to $\Lambda_{A_{0}}$ is a vector space isomorphism for every $A \in \mathcal{U}$.) Note that $P_{A_{0}}=\mathrm{Id}$ and $P_{A} \alpha \in \mathrm{T}_{A} \mathcal{L}$ iff $\alpha \in \mathrm{T}_{A_{0}} \mathcal{L}$. We claim that each operator $P_{A}$ extends to a Banach space automorphism of $\mathrm{T}_{A} \mathcal{A}^{k, p}(\Sigma)=W^{k, p}\left(\Sigma, \mathrm{T}^{*} \Sigma \otimes \mathfrak{g}\right)$ for all $k$ and $p$, and this automorphism depends smoothly on $A \in \mathcal{L}^{k, \infty}$. To prove this we write $P_{A}$ as the composition of three linear operators. The first is the Banach space isomorphism

$$
W^{k, p}\left(\Sigma, \mathrm{T}^{*} \Sigma \otimes \mathfrak{g}\right) \rightarrow \Lambda_{A_{0}} \times \Lambda_{A_{0}} \times W_{E}^{k+1, p}(\Sigma, \mathfrak{g}) \times W_{E}^{k+1, p}(\Sigma, \mathfrak{g})
$$

induced by the Hodge decomposition for $A_{0}$. Here $W_{E}^{k+1, p}(\Sigma, \mathfrak{g})$ denotes the $L^{2}-$ orthogonal complement of $E$ in $W^{k+1, p}(\Sigma, \mathfrak{g})$. The second operator is the restriction of $\Pi_{A}$ on the factors $\Lambda_{A_{0}}$ and is the identity on the factors $W_{E}^{k+1, p}(\Sigma, \mathfrak{g})$. We think of the target space of this second operator as the product

$$
W^{k, p}\left(\Sigma, \mathrm{T}^{*} \Sigma \otimes \mathfrak{g}\right) \times W^{k, p}\left(\Sigma, \mathrm{T}^{*} \Sigma \otimes \mathfrak{g}\right) \times W_{E}^{k+1, p}(\Sigma, \mathfrak{g}) \times W_{E}^{k+1, p}(\Sigma, \mathfrak{g}) .
$$

The third operator maps this product to $W^{k, p}\left(\Sigma, \mathrm{T}^{*} \Sigma \otimes \mathfrak{g}\right)$ via

$$
(\alpha, \beta, \xi, \eta) \mapsto \alpha+* \beta+\mathrm{d}_{A} \xi+* \mathrm{~d}_{A} \eta .
$$

The first operator is independent of $A$ and the third depends smoothly on $A \in \mathcal{L}^{k, \infty}$. By the Hodge decomposition for $A$ it restricts to an isomorphism from $\Lambda_{A} \times \Lambda_{A} \times$ $W_{E}^{k+1, p}(\Sigma, \mathfrak{g}) \times W_{E}^{k+1, p}(\Sigma, \mathfrak{g})$ to $W^{k, p}\left(\Sigma, \mathrm{T}^{*} \Sigma \otimes \mathfrak{g}\right)$. It remains to prove that the map

$$
\mathcal{U}^{k, \infty} \rightarrow \operatorname{Hom}\left(\Lambda_{A_{0}}, W^{k, p}\left(\Sigma, \mathrm{T}^{*} \Sigma \otimes \mathfrak{g}\right)\right): A \mapsto \Pi_{A}
$$

is smooth. To see this we write $\Pi_{A}$ as the composition of two projections

$$
\Pi_{A}=\left.\Pi_{H_{A, E}^{1}} \circ \Pi_{\mathrm{T}_{A} \mathcal{L}}\right|_{\Lambda_{A_{0}}} .
$$

Here $\Pi_{H_{A, E}^{1}}: W^{k, p}\left(\Sigma, \mathrm{T}^{*} \Sigma \otimes \mathfrak{g}\right) \rightarrow W^{k, p}\left(\Sigma, \mathrm{T}^{*} \Sigma \otimes \mathfrak{g}\right)$ denotes the $L^{2}$-orthogonal projection onto $H_{A, E}^{1}$ given by

$$
\Pi_{H_{A, E}^{1}} \alpha:=\alpha-\mathrm{d}_{A} \Delta_{A, E}^{-1}\left(\mathrm{~d}_{A}^{*} \alpha\right)+* \mathrm{~d}_{A} \Delta_{A, E}^{-1}\left(* \mathrm{~d}_{A} \alpha\right) .
$$


It depends smoothly on $A \in \mathcal{L}^{k, \infty} \cap \mathcal{U}$ since the same holds for the operator

$$
\Delta_{A, E}: W_{E}^{k+1, p}(\Sigma, \mathfrak{g}) \rightarrow W^{k-1, p}(\Sigma, \mathfrak{g}) / E
$$

and its inverse. The operator

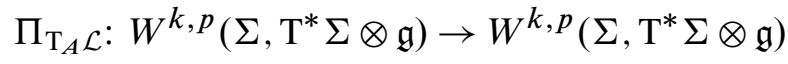

denotes the $L^{2}$-orthogonal projection onto $\mathrm{T}_{A} \mathcal{L}^{k, p}$. For $(k+1) p>2$ we know that $\mathcal{L}^{k, p} \subset \mathcal{A}^{k, p}(\Sigma)$ is a Banach submanifold, so $\Pi_{\mathrm{T}_{A} \mathcal{L}}$ depends smoothly on $A \in \mathcal{L}^{k, p}$, and this proves that $\Pi_{A}$ depends smoothly on $A \in \mathcal{L}^{k, \infty}$. In the case $(k+1) p \leq 2$, ie $k=0, p \leq 2$, we have $\mathcal{A}^{0,3}(\Sigma) \subset \mathcal{A}^{k, p}(\Sigma)$. The $L^{p}$ - and the $L^{3}$-norm are equivalent on the finite dimensional space $\Lambda_{A_{0}} \subset \Omega^{1}(\Sigma, \mathfrak{g})$. Hence $\Pi_{A}$ is the composition of the projection $\Pi_{\mathrm{T}_{A} \mathcal{L}}: L^{3}\left(\Sigma, \mathrm{T}^{*} \Sigma \otimes \mathcal{G}\right) \rightarrow L^{3}\left(\Sigma, \mathrm{T}^{*} \Sigma \otimes \mathcal{G}\right)$, restricted to $\Lambda_{A_{0}}$, the inclusion $L^{3}\left(\Sigma, \mathrm{T}^{*} \Sigma \otimes \mathcal{G}\right) \hookrightarrow L^{p}\left(\Sigma, \mathrm{T}^{*} \Sigma \otimes \mathcal{G}\right)$, and the projection $\Pi_{H_{A, E}^{1}}: L^{p}\left(\Sigma, \mathrm{T}^{*} \Sigma \otimes \mathcal{G}\right) \rightarrow L^{p}\left(\Sigma, \mathrm{T}^{*} \Sigma \otimes \mathcal{G}\right)$. All of these depend smoothly on $A \in \mathcal{L}^{0, \infty}$.

To prove (iv) shrink $\mathcal{U}$ such that $\left\|\mathbb{1}-P_{A}\right\|_{\mathcal{L}\left(L^{2}\right)} \leq 1 / 2$ for all $A \in \mathcal{U}$. Then $\lambda \mathbb{1}+$ $(1-\lambda) P_{A}$ is invertible on $L^{2}$ for every $\lambda \in[0,1]$ and every $A \in \mathcal{U}^{0, \infty}$. Invertibility on $W^{k, p}$ for $A \in \mathcal{U}^{k, \infty}$ now follows from elliptic regularity for the Laplace operator. This proves the theorem.

Theorem E.2 For every $A_{0} \in \mathcal{A}(Y, \mathcal{L})$ there is a neighbourhood $\mathcal{U} \subset \mathcal{A}(Y, \mathcal{L})$ of $A_{0}$ (open in the $\mathcal{C}^{0}$-topology) and a family of bijective linear operators

$$
Q_{A}: \Omega^{1}(Y, \mathfrak{g}) \rightarrow \Omega^{1}(Y, \mathfrak{g}),
$$

parametrized by $A \in \mathcal{U}$, such that the following holds.

(i) $Q_{A_{0}}=11$.

(ii) For every $A \in \mathcal{U}$ and every $\alpha \in \Omega^{1}(Y, \mathfrak{g})$ we have

$$
Q_{A} \alpha \in \Omega_{A}^{1}(Y, \mathfrak{g}) \quad \Longleftrightarrow \quad \alpha \in \Omega_{A_{0}}^{1}(Y, \mathfrak{g}) .
$$

Moreover, $\left.*\left(Q_{A} \alpha\right)\right|_{\partial Y}=\left.* \alpha\right|_{\partial Y}$ and $\left.\left(Q_{A} \alpha\right)\right|_{\partial Y}=0$ iff $\left.\alpha\right|_{\partial Y}=0$.

(iii) For every integer $k \geq 0$ and every $p>1$ the operator $Q_{A}$ extends to a Banach space isomorphism from $W^{k, p}\left(Y, \mathrm{~T}^{*} Y \otimes \mathfrak{g}\right)$ to itself; this extended operator depends smoothly on $A \in \mathcal{A}^{k, \infty}(Y, \mathcal{L})$ with respect to the operator norm on $Q_{A}$.

Proof Choose geodesic normal coordinates to identify a neighbourhood of $\partial Y$ with the product $(-\varepsilon, 0] \times \Sigma$ via an orientation preserving embedding

$$
\iota:(-\varepsilon, 0] \times \Sigma \rightarrow Y \text {. }
$$


For a connection $A \in \mathcal{A}(Y, \mathcal{L})$ and a 1 -form $\alpha \in \Omega^{1}(Y, \mathfrak{g})$ we write the pullbacks under $\iota$ in the form

$$
\iota^{*} A=: B(t)+\Psi(t) \mathrm{d} t, \quad \iota^{*} \alpha=: \beta(t)+\psi(t) \mathrm{d} t .
$$

Then $B(0)=\left.A\right|_{\Sigma} \in \mathcal{L}$. Choose a neighbourhood $\mathcal{U}_{0} \subset \mathcal{L}$ of $B_{0}:=\left.A_{0}\right|_{\Sigma}$ (open in the $\mathcal{C}^{0}$-topology) and an operator family $P_{B}: \Omega^{1}(\Sigma, \mathfrak{g}) \rightarrow \Omega^{1}(\Sigma, \mathfrak{g})$, parametrized by $B \in \mathcal{U}_{0}$, which satisfies the requirements of Theorem E.1. Then we have $P_{B_{0}}=11$. Now

$$
\mathcal{U}:=\left\{A \in \mathcal{A}(Y, \mathcal{L})|A|_{\Sigma} \in \mathcal{U}_{0}\right\}
$$

is a $\mathcal{C}^{0}$-open neighbourhood of $A_{0}$. For $A \in \mathcal{U}$ we define the bijective linear operator $Q_{A}: \Omega^{1}(Y, \mathfrak{g}) \rightarrow \Omega^{1}(Y, \mathfrak{g})$ by

$$
\iota^{*}\left(Q_{A} \alpha\right):=h(t) \beta(t)+(1-h(t)) P_{\left.A\right|_{\Sigma}} \beta(t)+\psi(t) \mathrm{d} t
$$

for $\iota^{*} \alpha$ of the form (147), and by $Q_{A} \alpha:=\alpha$ outside of the image of $\iota$. Here $h:(-\varepsilon, 0] \rightarrow[0,1]$ is a smooth cutoff function that vanishes near 0 and equals to 1 near $-\varepsilon$. The operator family $\left\{Q_{A}\right\}_{A \in \mathcal{U}}$ satisfies conditions (i)-(iii).

The construction of exponential maps will be based on the following.

Lemma E.3 Fix a constant $p>2$. There is an open neighbourhood

$$
\mathcal{U}^{0, p} \subset L^{p}\left(\Sigma, \mathrm{T}^{*} \Sigma \otimes \mathfrak{g}\right)
$$

of zero and a smooth map

$$
\mathcal{L}^{0, p} \times \mathcal{U}^{0, p} \rightarrow \mathcal{A}^{0, p}(\Sigma):(A, \alpha) \mapsto \Theta_{A}(\alpha)
$$

satisfying the following conditions:

(i) For every $A \in \mathcal{L}^{0, p}$ the map $\Theta_{A}: \mathcal{U}^{0, p} \rightarrow \mathcal{A}^{0, p}(\Sigma)$ is a diffeomorphism from $\mathcal{U}^{0, p}$ onto an $L^{p}$-open neighbourhood of $A$ in $\mathcal{A}^{0, p}(\Sigma)$ such that $\Theta_{A}(0)=A$ and $D \Theta_{A}(0)=\mathrm{Id}$. In particular, there is a uniform constant $C$ such that

$$
\begin{aligned}
\left\|\Theta_{A}(\alpha)-\Theta_{A}\left(\alpha^{\prime}\right)\right\|_{L^{p}} & \leq C\left\|\alpha-\alpha^{\prime}\right\|_{L^{p}} \\
\left\|D \Theta_{A}(\alpha) \beta-D \Theta_{A}\left(\alpha^{\prime}\right) \beta\right\|_{L^{p}} & \leq C\left\|\alpha-\alpha^{\prime}\right\|_{L^{p}}\|\beta\|_{L^{p}}
\end{aligned}
$$

for all $A \in \mathcal{L}^{0, p}, \alpha \in \mathcal{U}^{0, p}, \beta \in L^{p}\left(\Sigma, \mathrm{T}^{*} \Sigma \otimes \mathfrak{g}\right)$.

(ii) $\Theta$ is gauge equivariant in the sense that for $u \in \mathcal{G}^{1, p}(\Sigma)$

$$
\Theta_{u^{*} A}\left(u^{-1} \alpha u\right)=u^{*} \Theta_{A}(\alpha) .
$$


(iii) For every $A \in \mathcal{L}^{0, p}$

$$
\Theta_{A}\left(\mathrm{~T}_{A} \mathcal{L}^{0, p} \cap \mathcal{U}^{0, p}\right)=\mathcal{L}^{0, p} \cap \Theta_{A}\left(\mathcal{U}^{0, p}\right) .
$$

(iv) For every integer $k \geq 1$ and every $A \in \mathcal{L}^{k, p}$ the restriction of $\Theta_{A}$ to the intersection $\mathcal{U}^{k, p}:=\mathcal{U}^{0, p} \cap W^{k, p}$ is a diffeomorphism onto its (open) image in $\mathcal{A}^{k, p}(\Sigma)$. It depends smoothly on $A \in \mathcal{L}^{k, p}$ and satisfies

$$
\begin{aligned}
\left\|\Theta_{A}(\alpha)-\Theta_{A}\left(\alpha^{\prime}\right)\right\|_{W^{1, p}} & \leq C\left(1+\|A\|_{L^{\infty}}\right)\left\|\alpha-\alpha^{\prime}\right\|_{W^{1, p}}, \\
\left\|D \Theta_{A}(\alpha) \beta-D \Theta_{A}\left(\alpha^{\prime}\right) \beta\right\|_{W^{1, p}} & \leq C\left(1+\|A\|_{L^{\infty}}\right)\left\|\alpha-\alpha^{\prime}\right\|_{W^{1, p}}\|\beta\|_{W^{1, p}}
\end{aligned}
$$

for all $A \in \mathcal{L}^{1, p}, \alpha, \alpha^{\prime} \in \mathcal{U}^{1, p}$, and $\beta \in W^{1, p}\left(\Sigma, \mathrm{T}^{*} \Sigma \otimes \mathfrak{g}\right)$ with a uniform constant $C$.

(v) The restriction of $\Theta$ to an open neighbourhood of the zero section in the subbundle $* \mathrm{~T} \mathcal{L}^{0, p} \subset \mathcal{L}^{0, p} \times \mathcal{U}^{0, p}$ is a diffeomorphism onto an open neighbourhood $\mathcal{W}^{0, p} \subset \mathcal{A}^{0, p}(\Sigma)$ of $\mathcal{L}^{0, p}$. The composition of its inverse with the projection onto $\mathcal{L}^{0, p}$

$$
\pi: \mathcal{W}^{0, p} \rightarrow \mathcal{L}^{0, p}
$$

is gauge equivariant and maps $\mathcal{W}^{k, p}:=\mathcal{W}^{0, p} \cap W^{k, p}$ to $\mathcal{L}^{k, p}$ for every $k$.

Proof Since $\mathcal{L}^{0, p} / \mathcal{G}^{1, p}(\Sigma)$ is compact it suffices to provide the construction for smooth $A \in \mathcal{L}$. The smooth extension to $\mathcal{L}^{0, p}$ is then provided by the equivariance (ii). For every smooth connection $A \in \mathcal{L}$ we have an $L^{2}$-orthogonal direct sum decomposition from [33, Lemma 4.2],

$$
L^{p}\left(\Sigma, \mathrm{T}^{*} \Sigma \otimes \mathfrak{g}\right)=\mathrm{T}_{A} \mathcal{L}^{0, p} \oplus * \mathrm{~T}_{A} \mathcal{L}^{0, p} .
$$

Moreover, $\mathrm{T}_{A} \mathcal{L}^{0, p}=L_{A} \oplus \mathrm{d}_{A} W^{1, p}(\Sigma, \mathfrak{g})$, where $L_{A}:=\mathrm{T}_{A} \mathcal{L}^{0, p} \cap h_{A}^{1} \subset \Omega^{1}(\Sigma, \mathfrak{g})$ is the intersection of $\mathrm{T}_{A} \mathcal{L}$ with the harmonic (and thus smooth) 1-forms

$$
h_{A}^{1}:=\operatorname{kerd}_{A} \cap \operatorname{kerd}_{A}^{*} \subset \Omega^{1}(\Sigma, \mathfrak{g}) .
$$

We denote the $L^{2}$-orthogonal projection in (148) by

$$
\pi_{A}: L^{p}\left(\Sigma, \mathrm{T}^{*} \Sigma \otimes \mathfrak{g}\right) \rightarrow \mathrm{T}_{A} \mathcal{L}^{0, p} .
$$

It smoothly depends on $A \in \mathcal{L}$, is gauge equivariant $\pi_{u^{*} A}\left(u^{-1} \alpha u\right)=u^{-1} \pi_{A}(\alpha) u$, and satisfies $\mathrm{d}_{A}^{*} \circ \pi_{A}=\mathrm{d}_{A}^{*}$ because $\operatorname{im~}_{A} \subset \mathrm{T}_{A} \mathcal{L}^{0, p}$. By standard Hodge theory, this projection restricts to a bounded linear operator from the subspace $W^{k, p}\left(\Sigma, \mathrm{T}^{*} \Sigma \otimes \mathfrak{g}\right)$ to $\mathrm{T}_{A} \mathcal{L}^{k, p}=L_{A} \oplus \mathrm{d}_{A} W^{k+1, p}(\Sigma, \mathfrak{g})$ for every integer $k \geq 1$. For each $A \in \mathcal{L}$ the map

$$
\mathcal{L}^{0, p} \rightarrow \mathrm{T}_{A} \mathcal{L}^{0, p}: B \mapsto \pi_{A}(B-A)
$$


is smooth and its differential at $B=A$ is the identity. Hence it restricts to a diffeomorphism from an $L^{p}$-open neighbourhood of $A$ onto an open set

$$
\mathcal{V}_{A}^{0, p} \subset \mathrm{T}_{A} \mathcal{L}^{0, p}
$$

We denote its inverse by

$$
\psi_{A}: \mathcal{V}_{A}^{0, p} \rightarrow \mathcal{L}^{0, p}
$$

It follows immediately from the definition that $\psi$ is smooth and gauge equivariant in the sense that

$$
\psi_{u^{*} A}\left(u^{-1} \alpha u\right)=u^{*} \psi_{A}(\alpha)
$$

for all $A \in \mathcal{L}, u \in \mathcal{G}(\Sigma)$ and $\alpha \in \mathcal{V}_{A}^{0, p}$. Its differential at 0 is the identity, $D \psi_{A}(0)=\mathrm{Id}$, hence on a small ball $\left\{\|\alpha\|_{L^{p}} \leq \delta\right\} \supset \mathcal{V}_{A}^{0, p}$, we can bound the $L^{p}$-operator norm $\left\|D \psi_{A}(\alpha)\right\| \leq 2$, and thus obtain a linear estimate for all $\alpha, \alpha^{\prime} \in \mathcal{V}_{A}^{0, p}$

$$
\left\|\psi_{A}(\alpha)-\psi_{A}\left(\alpha^{\prime}\right)\right\|_{L^{p}} \leq \int_{0}^{1}\left\|D \psi_{A}\left(t \alpha+(1-t) \alpha^{\prime}\right)\right\|\left\|\alpha-\alpha^{\prime}\right\|_{L^{p}} \leq 2\left\|\alpha-\alpha^{\prime}\right\|_{L^{p}} .
$$

Similarly, since $D \psi_{A}$ is continuously differentiable, we obtain for all $\alpha, \alpha^{\prime}$ in (the possibly smaller) $\mathcal{V}_{A}^{0, p}$ and all $\beta \in \mathrm{T}_{A} \mathcal{L}^{0, p}$

$$
\left\|D \psi_{A}(\alpha) \beta-D \psi_{A}\left(\alpha^{\prime}\right) \beta\right\|_{L^{p}} \leq C\left\|\alpha-\alpha^{\prime}\right\|_{L^{p}}\|\beta\|_{L^{p}}
$$

with a uniform constant $C$. (In fact, $C$ is also independent of $A \in \mathcal{L}$ since the estimates are gauge invariant and $\mathcal{L} / \mathcal{G}(\Sigma)$ is compact). In particular, we have

$$
\left\|\psi_{A}(\alpha)-A\right\|_{L^{p}} \leq 2\|\alpha\|_{L^{p}}, \quad\left\|D \psi_{A}(\alpha) \beta-\beta\right\|_{L^{p}} \leq C\|\alpha\|_{L^{p}}\|\beta\|_{L^{p}} .
$$

Moreover, $\psi_{A}$ maps the intersection $\mathcal{V}_{A}^{k, p}:=\mathcal{V}_{A}^{0, p} \cap W^{k, p}$ to $W^{k, p}$-regular points in $\mathcal{L}^{k, p}$ because $F_{\psi_{A}(\alpha)}=0$ and

$$
\mathrm{d}_{A}^{*}\left(\psi_{A}(\alpha)-A\right)=\mathrm{d}_{A}^{*}\left(\pi_{A}\left(\psi_{A}(\alpha)-A\right)\right)=\mathrm{d}_{A}^{*} \alpha \in W^{k-1, p}(\Sigma, \mathfrak{g}) .
$$

In fact, we obtain an estimate for all $A \in \mathcal{L}^{1, p}, \alpha, \alpha^{\prime} \in \mathcal{V}_{A}^{1, p}$ (denoting all uniform constants by $C$ )

$$
\begin{aligned}
& \left\|\psi_{A}(\alpha)-\psi_{A}\left(\alpha^{\prime}\right)\right\|_{W^{1, p}} \\
& \leq C\left(\left\|\mathrm{~d}\left(\psi_{A}(\alpha)-\psi_{A}\left(\alpha^{\prime}\right)\right)\right\|_{p}+\left\|\mathrm{d}^{*}\left(\psi_{A}(\alpha)-\psi_{A}\left(\alpha^{\prime}\right)\right)\right\|_{p}+\left\|\psi_{A}(\alpha)-\psi_{A}\left(\alpha^{\prime}\right)\right\|_{p}\right) \\
& \leq C\left(\left\|\psi_{A}(\alpha)-\psi_{A}\left(\alpha^{\prime}\right)\right\|_{p}+\left\|\psi_{A}\left(\alpha^{\prime}\right)-A\right\|_{p}\right)\left\|\psi_{A}(\alpha)-\psi_{A}\left(\alpha^{\prime}\right)\right\|_{\infty} \\
& \quad+C\left\|\mathrm{~d}_{A}^{*}\left(\alpha-\alpha^{\prime}\right)\right\|_{p}+C\|A\|_{\infty}\left\|\psi_{A}(\alpha)-\psi_{A}\left(\alpha^{\prime}\right)\right\|_{p}+C\left\|\alpha-\alpha^{\prime}\right\|_{p} \\
& \leq C\left(1+\|A\|_{\infty}\right)\left\|\alpha-\alpha^{\prime}\right\|_{W^{1, p}}+C\left(\left\|\alpha-\alpha^{\prime}\right\|_{p}+\left\|\alpha^{\prime}\right\|_{p}\right)\left\|\psi_{A}(\alpha)-\psi_{A}\left(\alpha^{\prime}\right)\right\|_{W^{1, p}} .
\end{aligned}
$$


If we choose $\mathcal{V}_{A}^{0, p}$ sufficiently small, then the second term can be absorbed into the left hand side, which proves

$\left\|\psi_{A}(\alpha)-\psi_{A}\left(\alpha^{\prime}\right)\right\|_{W^{1, p}} \leq C\left(1+\|A\|_{L^{\infty}}\right)\left\|\alpha-\alpha^{\prime}\right\|_{W^{1, p}} \quad \forall A \in \mathcal{L}^{1, p}, \alpha, \alpha^{\prime} \in \mathcal{V}_{A}^{1, p}$.

Note that this estimate does not simply follow from smoothness of $\psi_{A}$ since $\mathcal{V}_{A}^{1, p}$ is not even bounded in the $W^{1, p}$-norm. Similarly, we obtain uniform estimates for the linearization $D \psi_{A}$ of $\psi_{A}$ using the identities $\mathrm{d}_{\psi_{A}(\alpha)}\left(D \psi_{A}(\alpha) \beta\right)=0=\mathrm{d}_{A} \beta$ and $\mathrm{d}_{A}^{*}\left(D \psi_{A}(\alpha) \beta\right)=\mathrm{d}_{A}^{*} \beta$,

$$
\begin{aligned}
& \left\|D \psi_{A}(\alpha) \beta-D \psi_{A}\left(\alpha^{\prime}\right) \beta\right\|_{W^{1, p}} \\
& \leq C \\
& \quad\left(\left\|\mathrm{~d}\left(D \psi_{A}(\alpha) \beta-D \psi_{A}\left(\alpha^{\prime}\right) \beta\right)\right\|_{p}+\left\|\mathrm{d}^{*}\left(D \psi_{A}(\alpha) \beta-D \psi_{A}\left(\alpha^{\prime}\right) \beta\right)\right\|_{p}\right. \\
& \left.\quad+\left\|D \psi_{A}(\alpha) \beta-D \psi_{A}\left(\alpha^{\prime}\right) \beta\right\|_{p}\right) \\
& \leq C\left(1+\|A\|_{\infty}\right)\left\|\left(D \psi_{A}(\alpha)-D \psi_{A}\left(\alpha^{\prime}\right)\right) \beta\right\|_{p}+\left\|\psi_{A}(\alpha)-\psi_{A}\left(\alpha^{\prime}\right)\right\|_{\infty}\left\|D \psi_{A}(\alpha) \beta\right\|_{p} \\
& \quad+C\left\|\psi_{A}\left(\alpha^{\prime}\right)-A\right\|_{p}\left\|D \psi_{A}(\alpha) \beta-D \psi_{A}\left(\alpha^{\prime}\right) \beta\right\|_{\infty} \\
& \leq C\left(1+\|A\|_{\infty}\right)\left\|\alpha-\alpha^{\prime}\right\|_{p}\|\beta\|_{p}+C\left\|\alpha-\alpha^{\prime}\right\|_{W^{1, p}}\left(1+\left\|\alpha^{\prime}\right\|_{p}\right)\|\beta\|_{p} \\
& \quad+C\|\alpha\|_{L^{p}}\left\|D \psi_{A}(\alpha) \beta-D \psi_{A}\left(\alpha^{\prime}\right) \beta\right\|_{W^{1, p}} .
\end{aligned}
$$

For $\mathcal{V}_{A}^{0, p}$ sufficiently small, this can be rearranged to

$$
\left\|D \psi_{A}(\alpha) \beta-D \psi_{A}\left(\alpha^{\prime}\right) \beta\right\|_{W^{1, p}} \leq C\left(1+\|A\|_{L^{\infty}}\right)\left\|\alpha-\alpha^{\prime}\right\|_{W^{1, p}}\|\beta\|_{L^{p}} .
$$

Now choose an open neighbourhood $\mathcal{U}^{0, p} \subset L^{p}\left(\Sigma, \mathrm{T}^{*} \Sigma \otimes \mathfrak{g}\right)$ of 0 such that

$$
\pi_{A}\left(\mathcal{U}^{0, p}\right) \subset \mathcal{V}_{A}^{0, p}
$$

for every $A \in \mathcal{L}$. Then the map $\Theta_{A}: \mathcal{U}^{0, p} \rightarrow \mathcal{A}^{0, p}(\Sigma)$ defined by

$$
\Theta_{A}(\alpha):=\psi_{A}\left(\pi_{A}(\alpha)\right)+\alpha-\pi_{A}(\alpha)
$$

has the required properties. The estimates for $\Theta_{A}$ follow from the linearity of $\pi_{A}$ and the linear estimates for $\psi_{A}$. To check (v) note that the differential of $\left.\Theta\right|_{* \mathrm{~T} \mathcal{L}^{0, p}}$ at $(A, 0)$ is the isomorphism $\mathrm{T}_{A} \mathcal{L}^{0, p} \times * \mathrm{~T}_{A} \mathcal{L}^{0, p} \rightarrow L^{p}\left(\Sigma, \mathrm{T}^{*} \Sigma \otimes \mathfrak{g}\right),(\eta, \beta) \mapsto \eta+\beta$. So the restriction of $\Theta$ to $* \mathrm{~T} \mathcal{L}^{0, p}$ is a local diffeomorphism near the zero section. To see that it is globally injective we assume by contradiction that $\Theta_{A_{i}}\left(\alpha_{i}\right)=\Theta_{B_{i}}\left(\beta_{i}\right)$ for some $A_{i}, B_{i} \in \mathcal{L}^{0, p}$ and some $\alpha_{i}, \beta_{i} \in * \mathrm{~T}_{A_{i}} \mathcal{L}^{0, p}$ with $\left\|\alpha_{i}\right\|_{L^{p}}+\left\|\beta_{i}\right\|_{L^{p}} \rightarrow 0$. Since $\Theta$ is equivariant and $\mathcal{L}^{0, p} / \mathcal{G}^{1, p}(\Sigma)$ is compact, we can assume w.l.o.g. $A_{i} \rightarrow A_{\infty}$ and $u_{i}^{*} B_{i} \rightarrow \widetilde{B}_{\infty}$ in the $\mathcal{C}^{\infty}$-topology for some $u_{i} \in \mathcal{G}^{1, p}(\Sigma)$. Then $\Theta_{A_{i}}\left(\alpha_{i}\right) \rightarrow A_{\infty}$ and $u_{i}^{*} \Theta_{A_{i}}\left(\alpha_{i}\right)=\Theta_{u_{i}^{*} B_{i}}\left(u_{i}^{-1} \beta_{i} u_{i}\right) \rightarrow \widetilde{B}_{\infty}$, so we can find a convergent subsequence $u_{i} \rightarrow u_{\infty} \in \mathcal{G}(\Sigma)$. Consequently $B_{i} \rightarrow u_{\infty}^{-1 *} \widetilde{B}_{\infty}=A_{\infty}$ has the same limit as $A_{i}$, in contradiction to the local injectivity of $\left.\Theta\right|_{* \mathrm{~T} \mathcal{L}^{0, p}}$. 
Theorem E.4 Fix a constant $p>2$ and a compact subset $N \subset \mathcal{A}^{1, p}(Y, \mathcal{L})$. Then there is an open neighbourhood $\mathcal{U} \subset \mathrm{T} \mathcal{A}^{1, p}(Y, \mathcal{L})$ of the zero section over $N$ and a smooth map

$$
\mathcal{U} \rightarrow \mathcal{A}^{1, p}(Y, \mathcal{L}):(A, \alpha) \mapsto E_{A}(\alpha)
$$

satisfying the following conditions:

(i) For every $A \in \mathcal{A}^{1, p}(Y, \mathcal{L})$ the map $E_{A}: \mathcal{U} \cap \mathrm{T}_{A} \mathcal{A}^{1, p}(Y, \mathcal{L}) \rightarrow \mathcal{A}^{1, p}(Y, \mathcal{L})$ is a diffeomorphism from a neighbourhood of 0 onto a neighbourhood of $A$ such that $E_{A}(0)=A$ and $\mathrm{d} E_{A}(0)=\mathrm{Id}$.

(ii) $E$ is gauge equivariant in the sense that for $u \in \mathcal{G}^{1, p}(Y)$

$$
E_{u^{*} A}\left(u^{-1} \alpha u\right)=u^{*} E_{A}(\alpha) .
$$

Proof Our construction will be based on the two maps from Lemma E.3,

$$
\Theta: \mathcal{L}^{0, p} \times \mathcal{U}^{0, p} \rightarrow \mathcal{A}^{0, p}(\Sigma), \quad \pi: \mathcal{W}^{0, p} \rightarrow \mathcal{L}^{0, p} .
$$

We start by fixing a tubular neighbourhood $\tau:(-1,0] \times \Sigma \hookrightarrow Y$ of the boundary $\partial Y \cong\{0\} \times \Sigma$ such that $\left.\tau^{*} A\right|_{\{t\} \times \Sigma} \in \mathcal{W}^{0, p}$ for all $A \in N$ and $t \in(-1,0]$. This is possible since $\tau^{*} N \subset W^{1, p}((-1,0] \times \Sigma) \subset \mathcal{C}^{0}\left((-1,0], \mathcal{A}^{0, p}(\Sigma)\right)$ is compact.

On the complement of the image of $\tau$ we define $E_{A}(\alpha):=A+\alpha$. On the image of $\tau$ write $\tau^{*} A=B(t)+\Psi(t) \mathrm{d} t$ and $\tau^{*} \alpha=\beta(t)+\psi(t) \mathrm{d} t$, where $\beta(t) \in \mathcal{U}^{0, p}$ can be ensured by the choice of neighbourhood $\mathcal{U} \ni \alpha$ of the zero section. With this we can define $\tau^{*} E_{A}(\alpha):=\widetilde{B}+(\Psi+\psi) \mathrm{d} t$ by

$$
\widetilde{B}(t):=B(t)+\rho(t)\left(\Theta_{\pi(B(t))}(\beta(t))-\pi(B(t))\right)+(1-\rho(t)) \beta(t),
$$

where $\rho:(-1,0] \rightarrow[0,1]$ is a smooth cutoff function satisfying $\rho \equiv 1$ near 0 and $\rho \equiv 0$ near -1 . The claimed properties of $E$ now simply follow from the properties of $\Theta$ and $\pi$ in Lemma E.3.

Corollary E.5 Let $B_{-}, B_{+} \in \mathcal{A}(Y, \mathcal{L})$ and $\Xi=A+\Phi \mathrm{d} s \in \mathcal{A}\left(\mathbb{R} \times Y, \mathcal{L} ; B_{-}, B_{+}\right)$. Fix $p>2$, then there is an open neighbourhood $\tilde{\mathcal{U}} \subset \mathrm{T}_{\Xi} \mathcal{A}^{1, p}\left(\mathbb{R} \times Y, \mathcal{L} ; B_{-}, B_{+}\right)$of zero such that

$$
\widetilde{E}: \tilde{\mathcal{U}} \rightarrow \mathcal{A}^{1, p}\left(\mathbb{R} \times Y, \mathcal{L} ; B_{-}, B_{+}\right), \quad \widetilde{E}(\alpha+\varphi \mathrm{d} s):=E_{A}(\alpha)+(\Phi+\varphi) \mathrm{d} s
$$

defines a continuously differentiable homeomorphism onto a neighbourhood of $\Xi$.

Proof Here we follow the construction of the exponential map of Theorem E.4 over the compact subset $N:=\{A(s) \mid s \in \mathbb{R}\} \cup\left\{B_{-}, B_{+}\right\} \subset \mathcal{A}(Y, \mathcal{L})$. We fix the tubular neighbourhood $\tau:(-1,0] \times \Sigma \hookrightarrow Y$ of the boundary such that $\tau^{*} A(s)=$ 
$B(s, t)+\Psi(s, t) \mathrm{d} t$ with $B(s, t) \in \mathcal{W}^{0, p}(\Sigma)$ for all $(s, t) \in \mathbb{R} \times(-1,0]$. For $\alpha+$ $\varphi \mathrm{d} s \in \mathrm{T}_{\Xi} \mathcal{A}^{1, p}\left(\mathbb{R} \times Y, \mathcal{L} ; B_{-}, B_{+}\right)$with $\|\alpha+\varphi \mathrm{d} s\|_{W^{1, p}(\mathbb{R} \times Y)}$ sufficiently small the Sobolev embedding $W^{1, p}(\mathbb{R} \times(-1,0] \times \Sigma) \hookrightarrow \mathcal{C}^{0}\left(\mathbb{R} \times(-1,0], L^{p}(\Sigma)\right)$ ensures that $\tau^{*} \alpha=\beta(s, t)+\psi(s, t) \mathrm{d} t$ with $\beta(s, t) \in \mathcal{U}^{0, p}$ for all $(s, t) \in \mathbb{R} \times(-1,0]$.

Thus we have $\widetilde{E}(\alpha+\varphi \mathrm{d} s)=A+\alpha+(\Phi+\varphi) \mathrm{d} s$ on $\mathbb{R} \times(Y \backslash \operatorname{im} \tau)$ and $\tau^{*} \widetilde{E}(\alpha+\varphi \mathrm{d} s)=$ $\widetilde{B}+(\Psi+\psi) \mathrm{d} t+(\Phi+\varphi) \mathrm{d} s$ on $\mathbb{R} \times(-1,0] \times \Sigma$ with

$$
\widetilde{B}(s, t)=B(s, t)+\rho(t)\left(\Theta_{\pi(B(s, t))}(\beta(s, t))-\pi(B(s, t))\right)+(1-\rho(t)) \beta(s, t) .
$$

That $\widetilde{E}$ is a bijection to a neighbourhood of $\Xi$ follows directly from Theorem E.4. For a restriction to a compact subset of $\mathbb{R} \times Y$ the smoothness of $\widetilde{E}$ follows directly from the smoothness of the 3-dimensional exponential map. To see that the 4-dimensional exponential map also is continuously differentiable with respect to the $W^{1, p}(\mathbb{R} \times Y)$ norm on the noncompact domain, it suffices to drop linear terms and the cutoff function $\rho$ and check that $\beta \mapsto \Theta_{\pi(B)}(\beta)-\pi(B)$ defines a $\mathcal{C}^{1}$-map $W^{1, p}\left(\mathbb{R} \times(-1,0], L^{p}(\Sigma)\right) \rightarrow$ $W^{1, p}\left(\mathbb{R} \times(-1,0], L^{p}(\Sigma)\right)$ and also induces a $\mathcal{C}^{1}$-map $L^{p}\left(\mathbb{R} \times(-1,0], W^{1, p}(\Sigma)\right) \rightarrow$ $L^{p}\left(\mathbb{R} \times(-1,0], W^{1, p}(\Sigma)\right)$. This follows from the linear bounds for $\Theta$ and $\pi$ in Lemma E.3, as follows. For all $\beta, \beta^{\prime} \in W^{1, p}\left(\mathbb{R} \times(-1,0] \times \Sigma, \mathrm{T}^{*} \Sigma \otimes \mathfrak{g}\right)$ we have

$$
\begin{gathered}
\left\|\Theta_{\pi(B(s, t))}(\beta(s, t))-\Theta_{\pi(B(s, t))}\left(\beta^{\prime}(s, t)\right)\right\|_{L^{p}(\Sigma)} \leq C\left\|\beta(s, t)-\beta^{\prime}(s, t)\right\|_{L^{p}(\Sigma)}, \\
\left\|\Theta_{\pi(B(s, t))}(\beta(s, t))-\Theta_{\pi(B(s, t))}\left(\beta^{\prime}(s, t)\right)\right\|_{W^{1, p}(\Sigma)} \leq C\left\|\beta(s, t)-\beta^{\prime}(s, t)\right\|_{W^{1, p}(\Sigma)} .
\end{gathered}
$$

For the $(s, t)$-derivatives we use the smoothness of $\Theta$ in the $L^{p}$-norm to obtain uniform continuity for the derivative by $A$ in the $L^{p}$-operator norm, ie $\| D_{1} \Theta(A, \alpha)-$ $D_{1} \Theta\left(A, \alpha^{\prime}\right)\|\leq C\| \alpha-\alpha^{\prime} \|_{L^{p}(\Sigma)}$ for all sufficiently small $\alpha, \alpha^{\prime} \in L^{p}\left(\Sigma, \mathrm{T}^{*} \Sigma \otimes \mathfrak{g}\right)$. Since $\|\beta(s, t)\|_{L^{p}(\Sigma)} \rightarrow 0$ for $s \rightarrow \pm \infty$ this applies for all $t \in(-1,0]$ and $|s|$ sufficiently large, so that

$$
\begin{aligned}
& \left\|\partial_{s}\left(\Theta_{\pi(B(s, t))}(\beta(s, t))-\Theta_{\pi(B(s, t))}\left(\beta^{\prime}(s, t)\right)\right)\right\|_{L^{p}(\Sigma)} \\
& \leq\left\|D \Theta_{\pi(B(s, t))}(\beta)\left(\partial_{s} \beta(s, t)-\partial_{s} \beta^{\prime}(s, t)\right)\right\|_{L^{p}(\Sigma)} \\
& \quad+\left\|\left(D_{1} \Theta(\pi(B(s, t)), \beta(s, t))-D_{1} \Theta\left(\pi(B(s, t)), \beta^{\prime}(s, t)\right)\right) \partial_{s} \pi(B(s, t))\right\|_{L^{p}(\Sigma)} \\
& \leq C\left(\left\|\partial_{s} \beta(s, t)-\partial_{s} \beta^{\prime}(s, t)\right\|_{L^{p}(\Sigma)}+\left\|\beta(s, t)-\beta^{\prime}(s, t)\right\|_{L^{p}(\Sigma)}\left\|\partial_{s} B(s, t)\right\|_{L^{p}(\Sigma)}\right) .
\end{aligned}
$$

(The same holds for $\partial_{t}(\ldots)$ ) Integrating these estimates over $(s, t) \in(-1,0] \times \mathbb{R}$ proves $W^{1, p}$-continuity of $\widetilde{E}(\alpha+\varphi \mathrm{d} s)$. To check continuity of the differential we use the analogous estimates for $D \Theta$, in particular we use uniform continuity for the second derivatives of $\Theta$ (which again hold for $\|\beta(s, t)\|_{L^{p}(\Sigma)}$ sufficiently small, ie $|s|$ 
sufficiently large) to obtain

$$
\begin{aligned}
& \left\|\partial_{s}\left(D \Theta_{\pi(B(s, t))}(\beta(s, t))-D \Theta_{\pi(B(s, t))}\left(\beta^{\prime}(s, t)\right)\right) \gamma(s, t)\right\|_{L^{p}(\Sigma)} \\
& \leq\left\|\left(D \Theta_{\pi(B)}(\beta)-D \Theta_{\pi(B)}\left(\beta^{\prime}\right)\right) \partial_{s} \gamma\right\|_{L^{p}(\Sigma)}+\left\|D^{2} \Theta_{\pi(B)}(\beta)\left(\partial_{s} \beta-\partial_{s} \beta^{\prime}, \gamma\right)\right\|_{L^{p}(\Sigma)} \\
& \quad+\left\|\left(D_{1} D_{2} \Theta(\pi(B), \beta)-D_{1} D_{2} \Theta\left(\pi(B), \beta^{\prime}\right)\right)\left(\partial_{s} \pi(B), \gamma\right)\right\|_{L^{p}(\Sigma)} \\
& \leq C \\
& \quad+\left\|\beta-\beta^{\prime}\right\|_{L^{p}(\Sigma)}\left\|\partial_{s} \gamma\right\|_{L^{p}(\Sigma)}+\left\|\partial_{s} \beta-\partial_{s} \beta^{\prime}\right\|_{L^{p}(\Sigma)}\|\gamma\|_{L^{p}(\Sigma)} \\
& \left.\quad+\left\|\beta-\beta^{\prime}\right\|_{L^{p}(\Sigma)}\left\|\partial_{S} B\right\|_{L^{p}(\Sigma)}\|\gamma\|_{L^{p}(\Sigma)}\right) .
\end{aligned}
$$

Integration then proves the continuity of $D \tilde{E}$ in $W^{1, p}(\mathbb{R} \times Y)$. (Strictly speaking, we can only integrate the above estimate over the complement of a compact interval in $\mathbb{R}$. However, the same estimate holds on the compact part due to the smoothness of $\Theta$.)

$$
\begin{aligned}
& \left\|\partial_{S}\left(D \Theta_{\pi(B)}(\beta)-D \Theta_{\pi(B)}\left(\beta^{\prime}\right)\right) \gamma\right\|_{L^{p}(\mathbb{R} \times(-1,0] \times \Sigma)} \\
& \leq C\left\|\beta-\beta^{\prime}\right\|_{L^{\infty}(\mathbb{R} \times(-1,0] \times \Sigma)}\left\|\partial_{S} \gamma\right\|_{L^{p}(\mathbb{R} \times(-1,0] \times \Sigma)} \\
& \quad+C\left\|\partial_{S} \beta-\partial_{s} \beta^{\prime}\right\|_{L^{p}(\mathbb{R} \times(-1,0] \times \Sigma)}\|\gamma\|_{L^{\infty}(\mathbb{R} \times(-1,0] \times \Sigma)} \\
& \quad+C\left\|\beta-\beta^{\prime}\right\|_{L^{\infty}(\mathbb{R} \times(-1,0] \times \Sigma)}\left\|\partial_{S} B\right\|_{L^{p}(\mathbb{R} \times(-1,0] \times \Sigma)}\|\gamma\|_{L^{\infty}(\mathbb{R} \times(-1,0] \times \Sigma)} \\
& \leq C\left\|\beta-\beta^{\prime}\right\|_{W^{1, p}(\mathbb{R} \times(-1,0] \times \Sigma)}\left(1+\left\|\partial_{S} B\right\|_{L^{p}(\mathbb{R} \times(-1,0] \times \Sigma)}\right)\|\gamma\|_{W^{1, p}(\mathbb{R} \times(-1,0] \times \Sigma)} .
\end{aligned}
$$

\section{References}

[1] S Agmon, A Douglis, L Nirenberg, Estimates near the boundary for solutions of elliptic partial differential equations satisfying general boundary conditions. I, Comm. Pure Appl. Math. 12 (1959) 623-727 MR0125307

[2] S Agmon, L Nirenberg, Lower bounds and uniqueness theorems for solutions of differential equations in a Hilbert space, Comm. Pure Appl. Math. 20 (1967) 207-229 MR0204829

[3] M F Atiyah, New invariants of 3- and 4-dimensional manifolds, from: "The mathematical heritage of Hermann Weyl (Durham, NC, 1987)", (R O Wells, Jr, editor), Proc. Sympos. Pure Math. 48, Amer. Math. Soc. (1988) 285-299 MR974342

[4] M F Atiyah, R Bott, The Yang-Mills equations over Riemann surfaces, Philos. Trans. Roy. Soc. London Ser. A 308 (1983) 523-615 MR702806

[5] M F Atiyah, V K Patodi, I M Singer, Spectral asymmetry and Riemannian geometry. I, Math. Proc. Cambridge Philos. Soc. 77 (1975) 43-69 MR0397797

[6] W Ballmann, J Brüning, G Carron, Dirac systems, in preparation

[7] B Booss-Bavnbek, K Furutani, The Maslov index: a functional analytical definition and the spectral flow formula, Tokyo J. Math. 21 (1998) 1-34 MR1630119 
[8] B Booss-Bavnbek, C Zhu, Weak symplectic functional analysis and general spectral flow formula arXiv:math.DG/0406139

[9] S K Donaldson, The orientation of Yang-Mills moduli spaces and 4-manifold topology, J. Differential Geom. 26 (1987) 397-428 MR910015

[10] S K Donaldson, Floer homology groups in Yang-Mills theory, Cambridge Tracts in Mathematics 147, Cambridge University Press (2002) MR1883043 With the assistance of M Furuta and D Kotschick

[11] S K Donaldson, P B Kronheimer, The geometry of four-manifolds, Oxford Mathematical Monographs, The Clarendon Press, Oxford University Press, New York (1990) MR1079726

[12] S Dostoglou, D A Salamon, Self-dual instantons and holomorphic curves, Ann. of Math. (2) 139 (1994) 581-640 MR1283871

[13] N Dunford, JTSchwarz, Linear operators II: spectral theory, Interscience (1964)

[14] A Floer, An instanton-invariant for 3-manifolds, Comm. Math. Phys. 118 (1988) 215-240 MR956166

[15] K Froyshov, On Floer homology and four-manifolds with boundary, PhD thesis, Oxford (1994)

[16] K Fukaya, Floer homology for 3-manifolds with boundary I (1997) Available at http://www . math.kyoto-u.ac.jp/\%7Efukaya/bdrt1.pdf

[17] A A Kirillov, A D Gvishiani, Theorems and problems in functional analysis, Problem Books in Mathematics, Springer, New York (1982) MR671088 Translated from the Russian by $\mathrm{HH}$ McFaden

[18] P Kirk, M Lesch, The $\eta$-invariant, Maslov index, and spectral flow for Dirac-type operators on manifolds with boundary, Forum Math. 16 (2004) 553-629 MR2044028

[19] P B Kronheimer, Four-manifold invariants from higher-rank bundles, J. Differential Geom. 70 (2005) 59-112 MR2192061

[20] P Kronheimer, T Mrowka, Monopoles and Three-Manifolds, New Mathematical Monographs 10, Cambridge University Press (2007)

[21] D McDuff, D Salamon, J-holomorphic curves and symplectic topology, Amer. Math. Soc. Colloquium Publ. 52, Amer. Math. Soc. (2004) MR2045629

[22] J W Milnor, Topology from the differentiable viewpoint, Based on notes by David W. Weaver, The University Press of Virginia, Charlottesville, Va. (1965) MR0226651

[23] T S Mrowka, K Wehrheim, $L^{2}$-topology and Lagrangians in the space of connections over a Riemann surface, in preparation

[24] J Robbin, Y Ruan, D A Salamon, The moduli space of regular stable maps, to appear in Math. Zeit. 
[25] J Robbin, D Salamon, The Maslov index for paths, Topology 32 (1993) 827-844 MR1241874

[26] J Robbin, D Salamon, The spectral flow and the Maslov index, Bull. London Math. Soc. 27 (1995) 1-33 MR1331677

[27] D Salamon, Spin geometry and Seiberg-Witten invariants, in preparation

[28] D Salamon, Lagrangian intersections, 3-manifolds with boundary, and the AtiyahFloer conjecture, from: "Proceedings of the International Congress of Mathematicians, Vol. 1, 2 (Zürich, 1994)”, Birkhäuser, Basel (1995) 526-536 MR1403953

[29] D Salamon, Lectures on Floer homology, from: "Symplectic geometry and topology (Park City, UT, 1997)", IAS/Park City Math. Ser. 7, Amer. Math. Soc. (1999) 143-229 MR1702944

[30] C H Taubes, Casson's invariant and gauge theory, J. Differential Geom. 31 (1990) 547-599 MR1037415

[31] C H Taubes, Unique continuation theorems in gauge theories, Comm. Anal. Geom. 2 (1994) 35-52 MR1312676

[32] K K Uhlenbeck, Connections with $L^{p}$ bounds on curvature, Comm. Math. Phys. 83 (1982) 31-42 MR648356

[33] K Wehrheim, Banach space valued Cauchy-Riemann equations with totally real boundary conditions, Commun. Contemp. Math. 6 (2004) 601-635 MR2078416

[34] K Wehrheim, Uhlenbeck compactness, EMS Series of Lectures in Mathematics, European Mathematical Society (EMS), Zürich (2004) MR2030823

[35] K Wehrheim, Anti-self-dual instantons with Lagrangian boundary conditions. I. Elliptic theory, Comm. Math. Phys. 254 (2005) 45-89 MR2116738

[36] K Wehrheim, Anti-self-dual instantons with Lagrangian boundary conditions. II. Bubbling, Comm. Math. Phys. 258 (2005) 275-315 MR2171696

[37] K Wehrheim, Lagrangian boundary conditions for anti-self-dual instantons and the Atiyah-Floer conjecture, Conference on Symplectic Topology, J. Symplectic Geom. 3 (2005) 703-747 MR2235859

Department of Mathematics, ETH

8092 Zürich, Switzerland

Massachusetts Institute of Technology, Department of Mathematics

77 Massachusetts Avenue, Cambridge, MA 02139-4307, USA

salamon@math.ethz.ch, katrin@math.mit.edu

Proposed: Tom Mrowka

Received: 19 July 2006

Seconded: Simon Donaldson, Eleny Ionel Accepted: 10 December 2007

Geometry 8 Topology, Volume 12 (2008) 